\title{
Optogenetic Control of Cardiac Arrhythmias
}

\author{
Dissertation \\ For THE AWARD OF THE DEGREE \\ "Doctor rerum naturalium" \\ of the University of Göttingen
}

within the doctoral program

Göttingen Graduate Center for Neurosciences, Biophysics, and Molecular Biosciences (GGNB), Physics of Biological and Complex Systems (PBCS)

by

Sayedeh Hussaini

from

Herat, Afghanistan

Göttingen, 2020 


\section{Thesis Committee}

Prof. Dr. Stefan Luther, Research Group Biomedical Physics, Max Planck Institute for Dynamics and Self-Organization

Prof. DR. JÖRG ENDERLEIN,

III. Physical Institute Biophysics, Georg-August-Universität Göttingen

Dr. Andreas Neef,

Center for Biostructural Imaging of Neurodegeneration, Max-Planck-Institute for Experimental Medicine

\section{Members of the Examination Board}

Referee: Prof. Dr. Stefan Luther,

Research Group Biomedical Physics,

Max Planck Institute for Dynamics and Self-Organization

$2^{\text {nd }}$ Referee: Prof. Dr. Jörg Enderlein,

III. Physical Institute Biophysics,

Georg-August-Universität Göttingen

Further members of the Examination Board:

Dr. Andreas NeEF,

Center for Biostructural Imaging of Neurodegeneration, Max-Planck-Institute for Experimental Medicine

Dr. Brett Carter,

Synaptic Physiology and Plasticity,

European Neuroscience Institute

Prof. Dr. Andre Fiala,

Molecular Neurobiology of Behavior,

Georg-August-Universität Göttingen

Prof. Dr. Stefan Klumpe,

Institute for the Dynamics of Complex Systems,

Georg-August-Universität Göttingen

Date of oral examination: 2021-02-05 
This thesis is dedicated to my parents, Mahrooseh $\&$ Masoud 



\section{Declaration}

I hereby declare that this thesis has been written independently and with no other sources and aids than quoted.

Sayedeh Hussaini

December 2021 



\begin{abstract}
The regular, coordinated contraction of the heart muscle is orchestrated by periodic waves generated by the heart's natural pacemaker and transmitted through the heart's electrical conduction system. Abnormalities occurring anywhere within the cardiac electrical conduction system can disrupt the propagation of these waves. Such disruptions often lead to the development of high frequency spiral waves that override normal pacemaker activity and compromise cardiac function. The occurrence of high frequency spiral waves in the heart is associated with cardiac rhythm disorders such as tachycardia and fibrillation. While tachycardia may be terminated by rapid periodic stimulation known as anti-tachycardia pacing (ATP), life-threatening ventricular fibrillation requires a single high-voltage electric shock that resets all the activity and restore the normal heart function. However, despite the high success rate of defibrillation, it is associated with significant side effects including tissue damage, intense pain and trauma. Thus, extensive research is conducted for developing low-energy alternatives to conventional defibrillation. An example of such an alternative is the low-energy anti-fibrillation pacing (LEAP). However, the clinical application of this technique, and other evolving techniques requires a detailed understanding of the dynamics of spiral waves that occur during arrhythmias.
\end{abstract}

Optogenetics is a tool, that has recently gained popularity in the cardiac research, which serves as a probe to study biological processes. It involves genetically modifying cardiac muscle cells such that they become light sensitive, and then using light of specific wavelengths to control the electrical activity of these cells. Cardiac optogenetics opens up new ways of investigating the mechanisms underlying the onset, maintenance and control of cardiac arrhythmias. In this thesis, I employ optogenetics as a tool to control the dynamics of a spiral wave, in both computer simulations and in experiments.

In the first study, I use optogenetics to investigate the mechanisms underlying defibrillation. Analogous to the conventional single electric-shock, I apply a single globally-illuminating light pulse to a two-dimensional cardiac tissue to study how wave termination occurs during defibrillation. My studies show a characteristic transient dynamics leading to the termination of the spiral wave at low light intensities, while at high intensities, the spiral waves terminate immediately.

Next, I move on to explore the use of optogenetics to study spiral wave termination via drift, theoretically well-known mechanism of arrhythmia termination in the 
context of electrical stimulation (e.g. ATP). I show that spiral wave drift can be induced by structured illumination patterns using lights of low intensity, that result in a spatial modulation of cardiac excitability. I observe that drift occurs in the positive direction of light intensity gradient, where the spiral also rotates with a longer period. I further show how modulation of the excitability in space can be used to control the dynamics of a spiral wave, resulting in the termination of the wave by collision with the domain boundary. Based on these observations, I propose a possible mechanism of optogenetic defibrillation.

In the next chapter, I use optogenetics to demonstrate control over the dynamics of the spiral waves by periodic stimulation with light of different intensities and pacing frequencies resulting in a temporal modulation of cardiac excitability. I demonstrate how the temporal modulation of excitability leads to efficient termination of arrhythmia. In addition, I use computer simulations to identify mechanisms responsible for arrhythmia termination for sub- and supra-threshold light intensities. My numerical results are supported by experimental studies on intact hearts, extracted from transgenic mice.

Finally, I demonstrate that cardiac optogenetics not only allows control of excitation waves, but also by generating new waves through the induction of wave breaks. We demonstrate the effects of high sub-threshold illumination on the morphology of the propagating wave, leading to the creation of new excitation windows in space that can serve as potential sites for re-entry initiation.

In summary, this thesis investigates several approaches to control arrhythmia dynamics using optogenetics. The experimental and numerical results demonstrate the potential of feedback-induced resonant pacing as a low-energy method to control arrhythmia. 


\section{Table of contents}

1 Introduction $\quad 1$

1.1 The Heart . . . . . . . . . . . . . . . . . . . . . . . . . 1

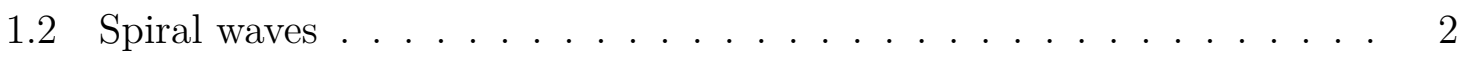

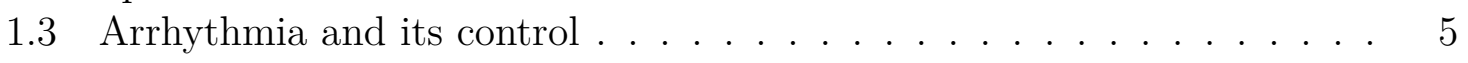

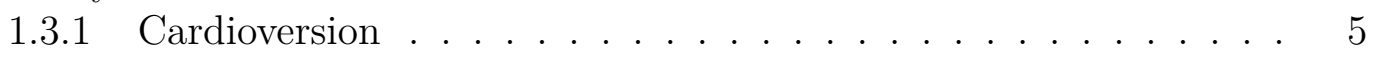

1.3.2 Defibrillation ................... 5

1.4 Prospective modalities for arrhythmia control . . . . . . . . . . 6

1.4.1 Electric-field-activated heterogeneities . . . . . . . . . . 6

1.4.2 Drift-induced termination . . . . . . . . . . . . 7

1.4.3 Optogenetics: Stimulation with light . . . . . . . . . 8

1.5 Cardiac optogenetics . . . . . . . . . . . . . . . . 9

1.6 Scope of this thesis . . . . . . . . . . . . . . . . . . . 11

2 Methods 13

2.1 Background . . . . . . . . . . . . . . . . . . 13

2.1.1 Cardiac action potential . . . . . . . . . . . 13

2.1.2 Excitation-Contraction coupling . . . . . . . . . . . 15

2.1.3 Mathematical description of the cardiac cell membrane . . . . 15

2.1.4 Cell-Cell coupling . . . . . . . . . . . . . . . . 16

2.2 Modelling Cardiac optogenetics . . . . . . . . . . . . . . . . . . 17

2.2.1 Spiral wave induction . . . . . . . . . . . . . . . 21

2.2.2 Detecting the phase singularity of a spiral wave . . . . . . . . 21

2.2.3 Determining the optical excitation threshold . . . . . . . . . . 23

2.2.4 Calculating curvature of a spiral wave's tip trajectory . . . . . . 24

2.3 Experimental methods . . . . . . . . . . . . . . 25

3 Control of arrhythmia using single supra-threshold optical pulse $\quad 27$

3.1 Scientific goal and findings . . . . . . . . . . . . . . . 27

3.1 Manuscript ........................ 28

4 Drift and termination of spiral waves in optogenetically modified $\begin{array}{ll}\text { cardiac tissue at sub-threshold illumination } & 39\end{array}$

4.1 Scientific goal and findings . . . . . . . . . . . . . . . . . 39

4.1.1 Manuscript . . . . . . . . . . . . . . . . . 40 
5 Feedback and non-feedback control of cardiac arrhythmia $\quad 61$

5.1 Scientific goal and findings . . . . . . . . . . . . . . . . . 61

5.1 .1 Manuscript . . . . . . . . . . . . . . . 62

6 Electric turbulence in cardiac tissue with sub-threshold modulation $\begin{array}{ll}\text { of excitability } & \mathbf{7 7}\end{array}$

6.1 Scientific goal and findings . . . . . . . . . . . . . . . . . 77

6.1.1 Manuscript ....................... 78

$\begin{array}{lll}7 & \text { Discussion and Conclusion } & 91\end{array}$

$\begin{array}{ll}\text { References } & 101\end{array}$ 


\section{Chapter 1}

\section{Introduction}

Life-threatening cardiac arrhythmias are associated with the occurrence of rotating spiral waves of electrical activity in the heart $[1,2]$. These abnormal waves can be most effectively eliminated by defibrillation, which involves complete electrical synchronization of the heart to restore its function [3]. Conventionally, defibrillation is achieved by applying high voltage electric shocks to the heart. However, this leads to a number of negative effects, such as severe pain, trauma and tissue damage, which compromise the high success rate of the method [4, 5]. Much research is devoted to the development of defibrillation techniques based on the use of low energy [6-9], but unfortunately, conventional defibrillation techniques do not work at low energies. Therefore, it is important to understand the mechanisms behind successful defibrillation at all energy levels. This requires a tool that can be used to study the mechanisms of arrhythmia control as well as its initiation in cardiac tissue. A possible candidate for such a tool, based on low energy, is optogenetics [10, 11]. Optogenetics has been around for more than a decade and has been used extensively to control the function of biological excitable systems such as the brain, skeletal muscle and heart with light and genetic modification [12-18]. Unlike conventional electrical methods, optogenetics provides cell type-specific stimulation with high spatial and temporal resolution $[19,20]$. In the cardiac community, this technique is currently being considered and investigated for low-energy defibrillation with patterned illumination [21-23]. In this thesis, I use simulations and wet experiments to study and control the dynamics of propagating spiral waves in light-responsive cardiac tissue.

\subsection{The Heart}

The heart is a vital organ in our body that supplies it with oxygen and nutrients by pumping up to 7200 litres of blood daily for an average healthy adult [24]. The electrical system of the heart, shown in Fig.1.1, initiates a coordinated contraction of the heart muscle, which causes blood to be pumped throughout the body. This electrical system consists of: 1) the sinoatrial (SA) node, 2) the atrioventricular (AV) node, 3) His bundle, and 4) the Purkinje fibers. An electrical signal is generated by the 


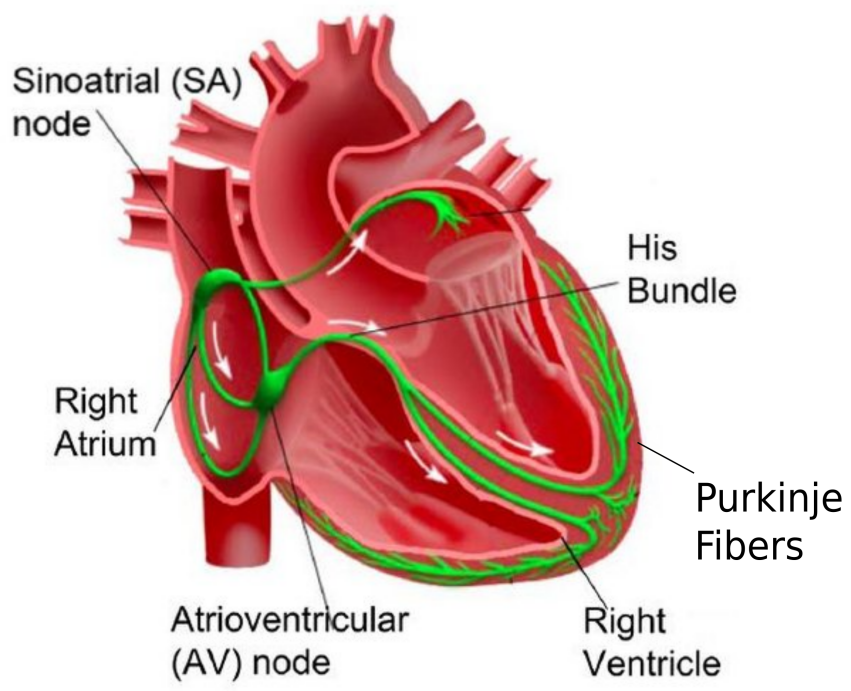

Figure 1.1 - The electrical system of the heart. This system consists of four main compartments through which electrical current flows. The four compartments are the SA node, the AV node, His bundle branches, and the Purkinje fibers [31].

SA node (pacemaker), which is located in the upper part of the right atrium [25-27]. This signal travels through the right and left atrial wall and ensures their coordinated contraction. Once the signal reaches the base of the right atrium, where the AV node is located, it is filtered into the His bundle after a short pause. The His bundle branches out into the Purkinje fibers, which run along the inside of the right and left ventricle and is connected to the innermost layer of the ventricular wall at discrete points called Purkinje ventricular junctions. The signal passes through the His-Purkinje System (HPS) and then excites the ventricles, resulting in their coordinated contraction. This entire process is called cardiac cycle [24, 27-30].

The electrical activity of the heart during a cardiac cycle can be monitored by the recordings in the electrocardiogram (ECG) (see Fig.1.2). During normal heartbeat, called sinus rhythm, the ECG signal consists of three main components, a P-wave that occurs during atrial contraction, a QRS complex that occurs during ventricular contraction, and a T-wave that occurs during ventricular relaxation [28]. Any deviation from this pattern, such as the presence of an additional wave in the signal, the lengthening or shortening of the period of a wave or the change in direction of the QRS complex, represents abnormal electrical activity of the heart and may be an indication of cardiac arrhythmia [27].

\subsection{Spiral waves}

The heart is a non-linear dynamical system capable of supporting the propagation of excitation waves [32-34]. Such a system is called an excitable medium. During normal heart function, these waves propagate such planar waves. Interruptions in the planar 


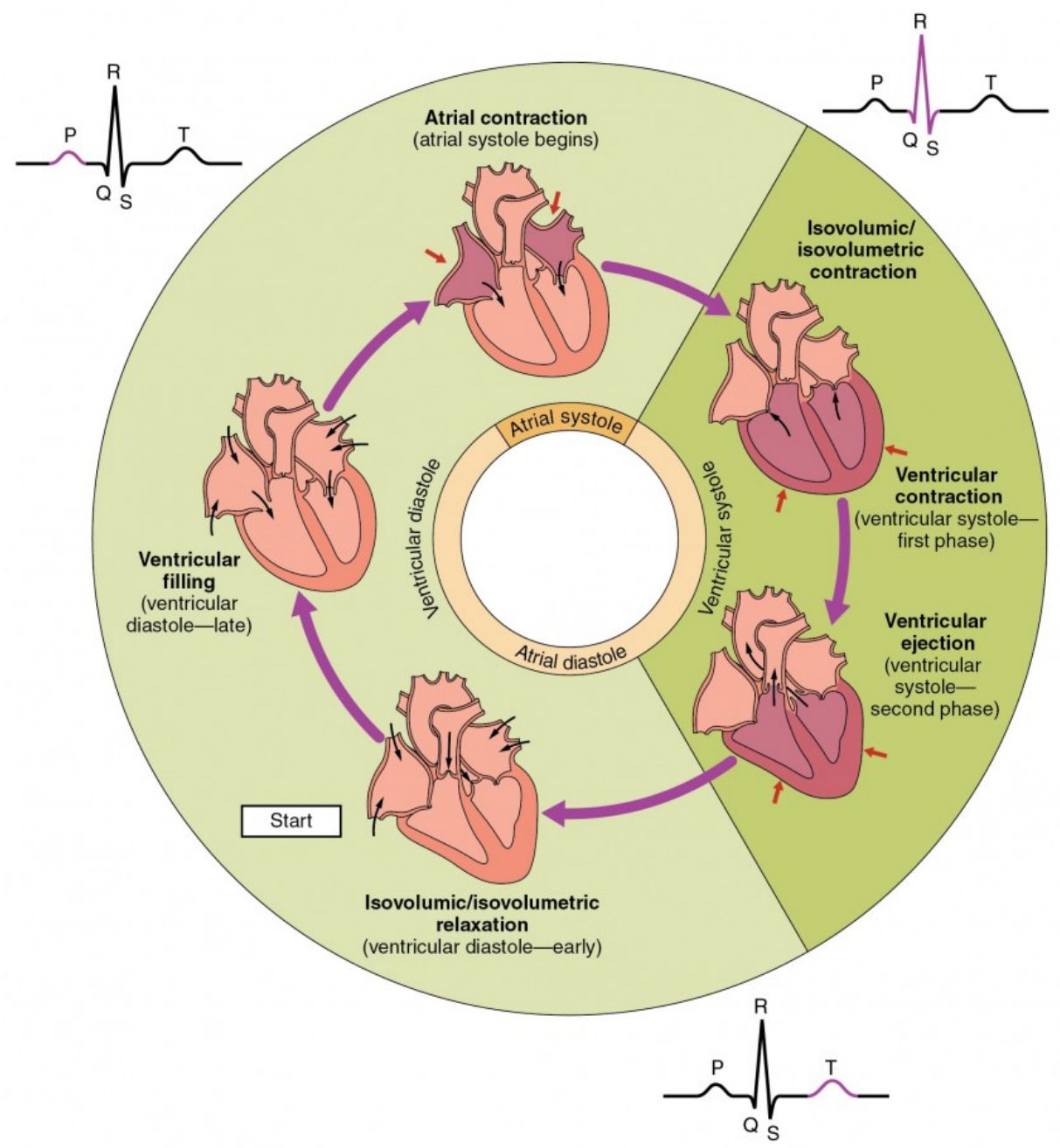

Figure 1.2 - Demonstration of the cardiac cycle with a normally beating heart. The ECG signal consists of three waves: The cycle begins with the atrial contraction (atrial systole), represented as a $P$-wave, and continues until the ventricular contraction (ventricular systole), represented as a $Q R S$ wave. Then it ends with the relaxation of the ventricle, represented as a T-wave. Taken from [27], with Creative Commons License.

propagation waves caused by the presence of a structural or functional inhomogeneity, such as tissue scarring, induced by a myocardial infarction, can lead to the development of wave breaks that have the potential to mature into spiral waves [35-38]. Since more than a century ago, through the pioneering works of $\mathrm{G}$. R. Mines $[1,2,39]$ and W. E. Garrey [40] on the identification of cardiac arrhythmia mechanisms, circulatory movements of excitation waves in the heart tissue were associated with the occurrence of cardiac arrhythmias. Fig.1.3 shows a possible way of spiral wave formation. It illustrates the development of a wave break near a heterogeneity (shown in a red dashed rectangle) with a long refractory period (a certain time that the system needs to recover and re-excitation is not possible). Two planar waves (wave 1 and wave 2) are induced 


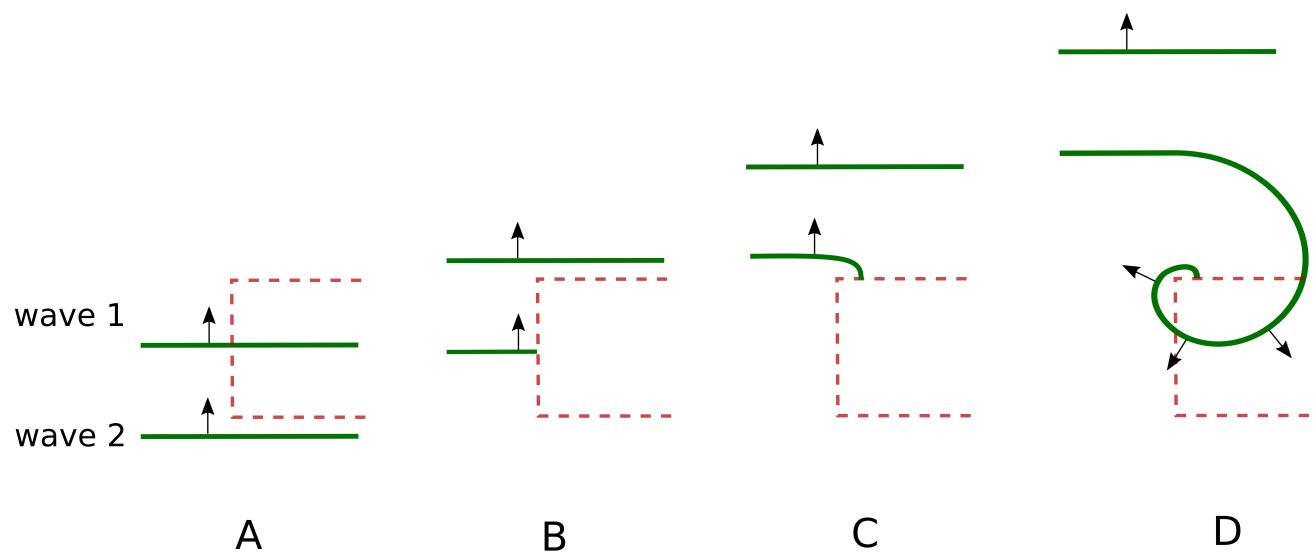

Figure 1.3 - Formation of a spiral wave around a heterogeneity. The area indicated by a red dashed line shows a heterogeneity with a high refractory time. A) Two planar waves, wave 1 and wave 2, shown with a solid line, spread out in one area. B) Although the wave 1 propagates through heterogeneity, the wave 2 does not enter the heterogeneous domain because of its prolonged refractory time. This results in wave break. C) It shows the development of the right edge of the planar wave to a spiral wave. D) It shows the complete formation of the spiral wave and its rotation after the refractory period. Taken from [35], with permission.

by external electrical stimulation. When wave 1 passes through the heterogeneity (Fig.1.3-A), it slows down at the site of the heterogeneity, which gives it its longer refractory time compared to the rest of the range. This interrupts the propagation of wave 2 (Fig.1.3-B), causing it to break and form a spiral wave (Fig.1.3-C). When the propagating planar wave is interrupted, a break typically occurs at the point of interruption. As the refractory period disappears in the heterogeneity, the spiral wave develops through the heterogeneous region. This leads to the formation of a spiral wave within the domain, as shown in Fig.1.3-D. In order to characterize a spiral wave, several parameters are considered: e.g. its period(s), its wavelength, the trajectory of its tip, the size and shape of its core (a region at the center of a spiral wave), and its stability (periodic rotation of a spiral wave) [37, 41, 42]. Some excitable media support a single spiral wave that rotates periodically, quasiperiodically (with several fundamental frequencies), or chaotically (i.e., with a broad spectrum of excitation frequencies), while others support spirals only temporarily [37, 42]. In such media, spiral waves cannot sustain themselves and end within a few revolutions. The heart tissue is an example of an excitable medium that shows the both phenomena, i.e. the long-term maintenance of spiral waves and the self-termination [43, 44]. The presence of spiral wave (in two-dimensional cardiac tissue) or scroll wave (in three-dimensional cardiac tissue) activity in a heart disrupts heart function and causes cardiac arrhythmia. It is therefore very important to study all facets of spiral/scroll waves rotating in a heart tissue. 


\subsection{Arrhythmia and its control}

During an arrhythmias, either of three situations can arise: 1) the heart beats very fast, thereby exceeding its normal physiological capacity, 2) the heart slows down to extremely low rates below minimum physiological demand, or 3) the heart beats irregularly at high frequency and low efficiency. The first case is the characteristic of ventricular tachycardia (VT) $[45,46]$, where the electrical activity is driven by a single rotating spiral wave. The second case is observed during bradycardia [47, 48], whereas, the third case is typical for ventricular fibrillation (VF) [49-51]. Here, the electrical activity in the heart organizes into multiple spiral waves or dynamically emerging and disappearing wave fragments.

Current clinical methods for controlling cardiac arrhythmias include cardioversion and defibrillation $[52,53]$.

\subsubsection{Cardioversion}

Electrical cardioversion and chemical cardioversion help to convert abnormal heart rhythms into a normal rhythm by providing synchronized feedback to the electrical signal generated by the heart. In electrical cardioversion, electrical shocks are delivered to the heart via electrodes placed on the patient's chest, which are synchronized with the electrical signal of the ECG display. These pulses reset all electrical activity in the ventricles to restore normal rhythm. In chemical cardioversion, antiarrhythmic drugs are used to correct a disturbed heart by blocking certain cell membrane proteins such as ion channels and influencing the electrical current which makes the heart beat [54-56].

\subsubsection{Defibrillation}

Defibrillation is used in emergencies and under severe conditions. It delivers nonsynchronized random electric shocks to the cardiac tissue to restore the sinus rhythm. This treatment depolarizes a critical amount of the tissue and resets the entire heart, causing the sinus node to begin pulsing regularly. Defibrillators can be external (Automated External Defibrillator (AED)) [57-59] or internal (Implantable Cardioverter Defibrillators (ICDs)) [60]. AEDs are portable and battery-powered devices that transmit electrical shocks externally through the electrodes attached to the chest, while ICDs are surgically placed in the chest, connected to wires that detect the heart rate and deliver a shock when needed [56, 60].

However, even the state of the art treatment modalities suffer from suboptimality as they usually come with a number of unwanted side effects such as pain, trauma, tissue damage, anxiety, and depression $[4,5]$. It is for this reason that cardiac researchers are constantly looking for new approaches to control arrhythmias; approaches that work with low energy and cause less pain $[4,5,61,62]$. So far, the only known low energy method that has been successfully translated to the clinic is anti-tachycardia pacing (ATP). Here, a train of weak electric pulses are applied with a frequency higher than 
the frequency of the arrhythmia (tachycardia), in order to drive a single spiral wave toward the unexcitable atrioventricular limit in favor of termination [63-68]. However, this approach fails in case of arrhythmias that involve very high frequency and/or multiple spiral waves [69]. Thus, there is a growing demand for a clinical method that can be used to control fibrillation.

\subsection{Prospective modalities for arrhythmia control}

\subsubsection{Electric-field-activated heterogeneities}

Many theoretical and numerical studies have shown that intrinsic heterogeneities (such as blood vessels) of the heart tissue are capable of generating excitation waves when a suitable external electric field is applied [69-74]. These heterogeneities are called wave emission heterogeneities (WEH) [69] or virtual electrodes [74]. The application of a sufficient electric field to the heart tissue creates areas of depolarization (when the membrane potential is positive) and hyperpolarization (when the membrane potential is less than resting membrane voltage) around the virtual electrodes. This results in the propagation of an excitation wave from the depolarized region through the cardiac tissue $[3,75]$.
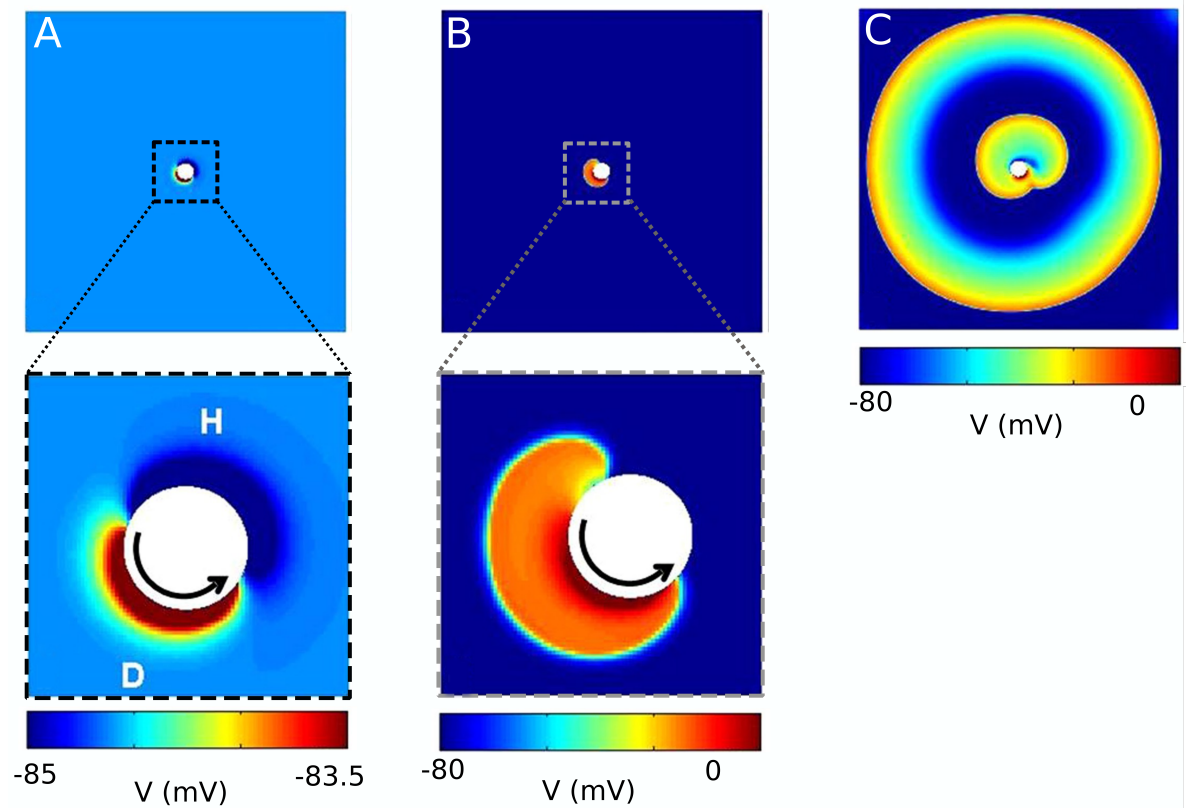

Figure 1.4 - Propagation of an excitation wave out of a heterogeneity. (A-C) Time lapse sequence of the wave as it emerges from a circular heterogeneity (white) located at the centre of the simulation domain. Insets in $(A)$ and $(B)$ show enlarged images of the emergent wave. $D$ and $H$ indicate regions of depolarisation and hyperpolarization, respectively, near the heterogeneity that trigger initiation of the wave. (C) Emergence of a second wave from the heterogeneity, caused by the application of an external electric field. $V$ is the membrane voltage. Taken from [76], an open access article. 
Fig.1.4 shows two different regions: hyperpolarization $(\mathrm{H})$ and depolarization (D) which are formed around an obstacle, shown as a white circle, under a certain strength of an external electric field. This leads to the propagation of a sequence of excitation waves through the entire domain. The number of such activated electrodes can be regulated by controlling the electric field strength. Therefore, these electrodes seem to provide a better control of higher frequency and multiple spiral-associated arrhythmias. A study by S. Luther and F. Fenton et al. [6] has experimentally shown the reaction of the heterogeneities of a scale-free coronary vessel tissue to the electric field. They demonstrated the activation of cardiac tissue heterogeneities as nucleation sites under the influence of the pulsed electric field. The propagation of intramural excitation waves from these heterogeneities can drive the spatio-temporal dynamics of the waves near their cores and thus lead to their termination by mutual collision or collision with an inexcitable boundary. These experimental observations suggest novel control strategies, such as low-energy anti-fibrillation pacing (LEAP) [6], which requires low-energy stimulation to terminate fibrillation $[7,8,77]$.

\subsubsection{Drift-induced termination}

Numerous theoretical and computational studies have shown that ionic-type heterogeneities, which are associated with a non-uniform recovery from the excited state [78-81], can cause a spiral wave to drift in cardiac tissue [82-87]. This drift has a temporal component, which is related to a change in the rotational frequency of the spiral wave, and a spatial component, which is related to the movement of the center of rotation or the core of the spiral wave. The drift due to the heterogeneities of the heart tissue can lead to self-termination or the initiation more spiral waves [82, 84, 85]. Applying an external stimulation such an electrical field might also induces spiral wave drift, which may lead to its termination [88-91]. Algadze et al. [92] and Mikhailov et al. [93] suggested that global temporal modulation of the excitability of the domain can cause a spiral wave to drift, as shown in Fig.1.5 A-B.

Numerical studies by V. Biktashev et al. [94] and S. Morgan et al. [95] show high efficiency of arrhythmia termination when resonant stimulation is applied to a spiral wave. In resonant stimulation, a series of electrical pulses is applied at a frequency close to the frequency of the arrhythmia. However, this technique works as long as the spiral wave rotates at a fixed frequency. Due to the change of the spiral wave frequency near the inexcitable boundary [96], the spiral wave is no longer triggered resonantly. This causes the spiral wave to turn and drift away from the boundary, resulting in no collision with the boundary. This phenomenon shows that stimulating the spiral wave with a fixed frequency is not the best strategy because the frequency of the spiral wave could be influenced by the excitable boundary or inhomogeneity present in the heart tissue $[94,96]$. A possible solution is to change the stimulation frequency based on the most recent frequency of the spiral wave using a feedback method. The feedback algorithm is a robust method that can trigger a pulse based on updated information from a measurement electrode that records the arrhythmia frequency. In this method, a pulse is triggered when the wavefront touches this measurement point. Therefore, this 
method can be used to rectify the barriers that can influence the arrhythmia frequency, such as heterogeneities and unbreakable boundaries, and to constantly update the arrhythmia frequency. Figs.1.5 D-F indicate the reflection of the spiral wave at the boundary by resonant stimulation. Figs.1.5 G-I, on the other hand, illustrate the collision of the same spiral wave with the boundary by the method of feedback pacing.

A

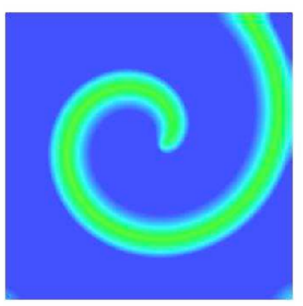

D

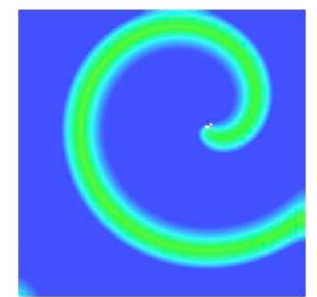

G

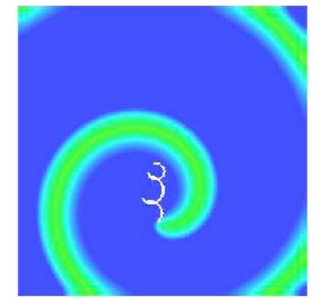

B

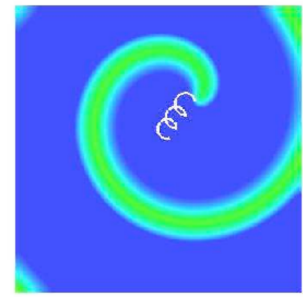

E

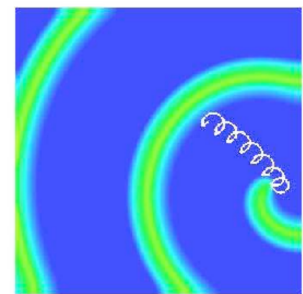

$\mathrm{H}$

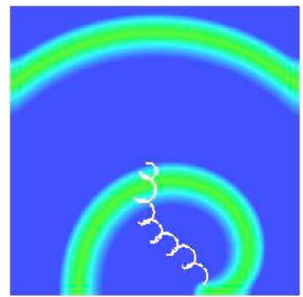

$\mathrm{C}$

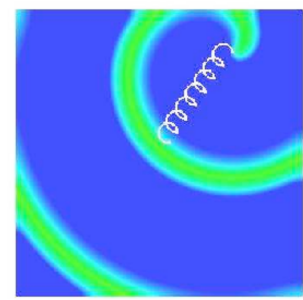

$\mathrm{F}$
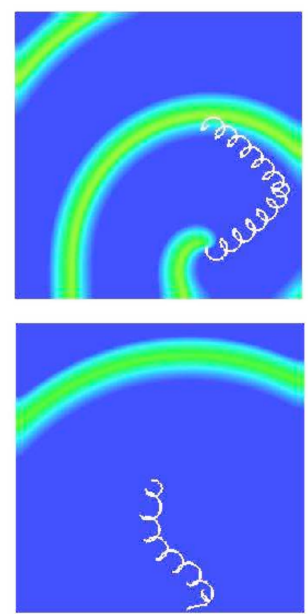

Figure 1.5 - Drift induced by resonance and feedback pacing. A-C) show a resonant drift of a spiral wave via an external periodic pacing at a frequency close to the spiral wave frequency. $D-F$ ) show an initial resonant drift of the spiral wave, which is repelled by the boundary due to the change of the spiral wave frequency near the boundary. G-I) show an inducing drift and collision of the wave with the boundary using the feedback stimulation method. Taken from [95], with permission.

\subsubsection{Optogenetics: Stimulation with light}

Optogenetics is a biological technique that involves the genetic modification of normally light-insensitive cells to enable control of their electrical activity with light. It was first used in neuroscience to study and control the electrical activity of neurons. The aim was to gain deeper insights into the functioning of the brain [12-15, 97, 98]. The method involves artificially expressing a light-gated ion channel on the cell membrane of these cells which would allow light-controlled passage of ions across the cell membrane [12-15, 98-100], with a temporal precision of a few milliseconds [20, 101]. Typically, the light-responsive ion channel, which is expressed on the cell membrane in optogenetics, comes from a family of light-responsive proteins, the Opsins, e.g. Channelrhodopsin, 
Halorhodopsin etc. These proteins are incorporated in the cell membrane through a variety of methods, such as viral vector release, electroporation etc. [19, 102-104]. The available opsins are able to either depolarize or hyperpolarize the cells by allowing a specific type of ion to pass through the membrane. This leads to a bidirectional control of the cellular activity, excitation or silence of an excitable cell. Compared to conventional electrical methods for controlling cardiac arrhythmias, optogenetics has shown tangible advantages such as: a long stimulation time in the order of milliseconds, very high spatial and termporal precision, the stimulation of certain cell types, and painless stimulation [19, 21, 99, 105]. Furthermore, the combination of this technique with optical imaging [106-108], a technique to study cardiac electrophysiology by recording e.g. membrane voltage signal, can be an effective approach to detect electrical activity while stimulating excitable tissue [20, 101].

\subsection{Cardiac optogenetics}

The cardiac cell membrane consists of a lipid bilayer that separates the interior of the cell from its exterior. Various types of proteins, such as ion channels, exchangers and pumps are embedded within this membrane [109, 110]. The presence of these proteins causes the membrane to act as a diffusion barrier, allowing ions to migrate through the membrane under certain conditions [111-113]. Channelrhodopsin-2 (ChR2) and Natromonas pharaonis halorhodomspin (NpHR) are two common photosensitive proteins that respond to light of specific wavelengths $[114,115]$. The ChR2 found in the alga Chlamydomonas reinhardtii can be expressed in mammalian cardiomyocytes, to cause cellular depolarization in the presence of a blue light $[114,116,117]$.

$\mathrm{NpHR}$ is a chloride pump that drives hyperpolarization in response to yellow light [118-120]. This pump acts as an inhibitor, generating an inward chloride current, which reduces the membrane potential to more negative values and suppresses cardiomyocyte activity, as shown in Fig.1.6-B. Other types of inhibitors include proton pumps, which are activated by green light, e.g, Arch/eArch(from Halorubrum sodomense). These generate outward currents of hyperpolarizing protons, which also silence cellular activity $[121,122]$.

Two experimental studies in 2010, one on the modulation of heart function in mice [99] and the other in zebrafish [100], demonstrated the potential of this technique in cardiac research. Since then, optogenetics has been used in a large number of experimental and numerical studies on the electrophysiology of the heart, from the single cell to the whole organ, with the aim of developing a deeper understanding of arrhythmia dynamics and its control [10, 11, 21-23, 123, 124]. Furthermore, this technique is promising for efficient control of cardiac arrhythmias, and is potentially less harmful than current conventional methods [21, 125].

Recent studies on optogenetic defibrillation in the atria and ventricles point to its possible clinical potential. At the organ level, some studies demonstrated the possibility to terminate ventricular arrhythmias ex vivo using local, structured and global epicardial illumination $[21-23,99,126]$. Recently, the first autogenic arrhythmia 

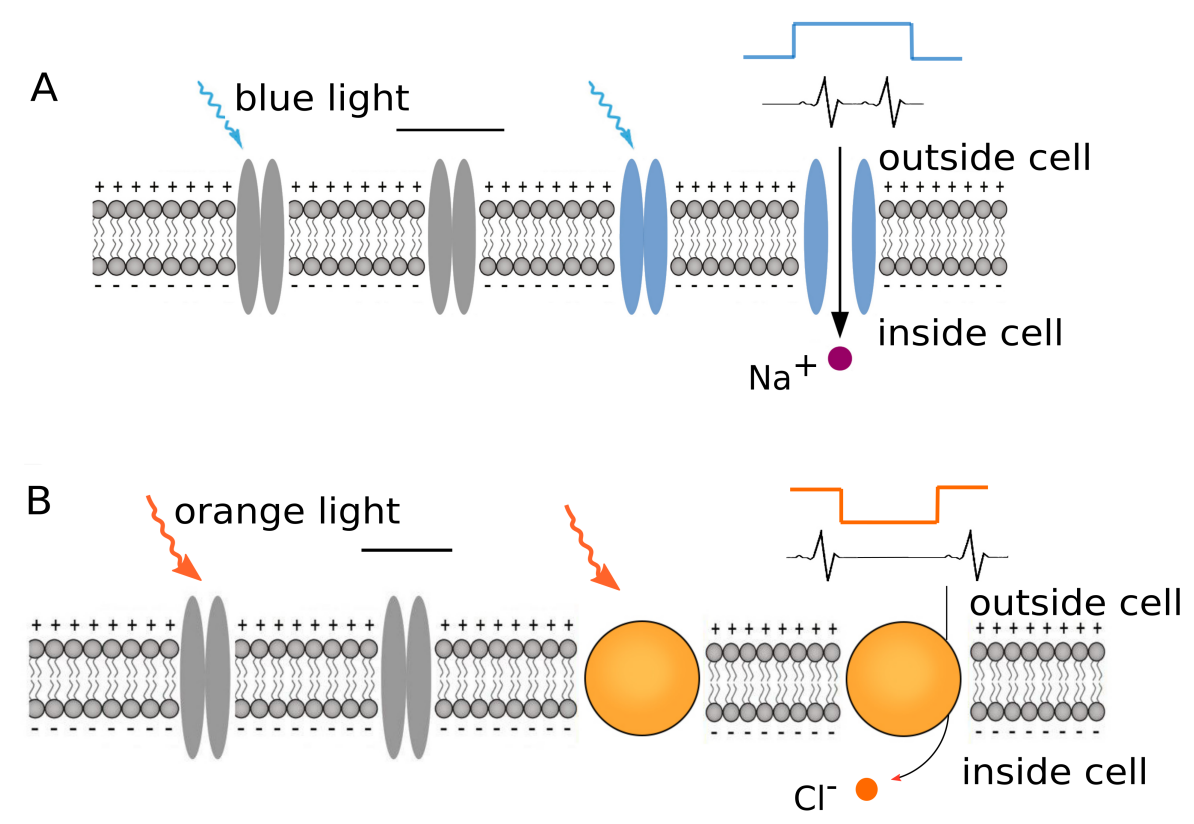

Figure 1.6 - Schematic representation of photosensitive proteins. A) A cell membrane consists of a normal channel (not photosensitive), which is shown in grey and ChR2. When blue light is applied to the cell expressed by ChR2, the cell is excited and depolarized. B) A cell membrane contains a normal channel shown in gray and NpHR. When yellow light is applied to the cell expressed by NpHR, the cell is inhibited and hyperpolarized.

termination system was developed in an optogenetically-modified rat heart (atria) with an implantable light source and a rhythm detector that functioned under ex vivo, in vivo and closed thoracic conditions [125]. These results suggest that this technique can be a painless technique compared to the conventional methods, but experiments on larger animals are still warranted [125].

From another perspective, optogenetics can also be used as a tool to study the mechanisms underlying the initiation and control of spiral wave dynamics during an arrhythmia. Some numerical and experimental studies demonstrated how optogenetics can be used to investigate the fundamental dynamical properties of spiral waves, such as chirality, attraction, pinning, and unpinning [10,11]. These studies illustrate the applicability of optogenetics to basic scientific research. In all of these studies, spiral wave dynamics has been controlled using supra-threshold illumination. Such illumination triggers the occurrence of an action potential in a cell, and leads to the propagation of new excitation waves in extended media. In this thesis, I use simulations and wet-lab experiments to investigate approaches to control spiral wave dynamics in cardiac tissue with optogenetic modification. I explore spiral wave dynamics not only with supra-threshold illumination, but also with sub-threshold light stimulation, which is known to cause a modest elevation of the cell membrane voltage, without invoking new action potentials. My studies show that sub-threshold illumination can be used strategically to observe all kinds of dynamical effects of the spiral wave including drift, 
meander, breakup and termination.

\subsection{Scope of this thesis}

The general aim of the work presented in this thesis is to study and control the dynamics of spiral waves in cardiac tissue. The main mode of investigation was computer simulations. The numerical studies led to the establishment of the ongoing experiments to control arrhythmia dynamics in the intact mouse heart, ex vivo, using global illumination perturbations.

This thesis is divided into seven chapters and is organized as follows:

1) Introduction. Here I give a general overview of the problem, its scientific relevance and the link to the basic and translational science. I provide essential information necessary for developing a better understanding of the work presented in the following chapters.

2) Methods. Here, I begin with a general background on cardiac electrophysiology. I describe the mathematical equivalent of ion transport across the cell membrane, using ordinary differential equations, and introduce the formulation used throughout the thesis to model the behaviour of transmembrane voltage across a single cardiac cell membrane. I then extend the model to two dimensions using partial differential equations to describe electrical signal propagation through cardiac tissue. Furthermore, I describe the different analysis techniques that I used.

Chapters 3-6 are devoted to descriptions of results obtained in the course of my studies. In each chapter, I address a specific research question using numerical and experimental investigations.

3) In chapter three, I investigate the termination rate of an arrhythmia by applying a single global supra-threshold optical pulse at different pulse lengths and light intensities in analogy to the conventional single-shock defibrillation method. The question addressed in this chapter is: How comparable is the mechanism of arrhythmia control with a single global optical pulse to the conventional single-shock defibrillation method?

4) Chapter four addresses the question: Can the dynamics of arrhythmia be controlled using sub-threshold illumination? For this purpose, different sub-threshold illumination patterns are applied to the system. These patterns modulate the spatial excitability of the system, leading to a control of the dynamics of the arrhythmia in favor of its termination. Furthermore, the numerical observations lead to the postulation of a new underlying mechanism for the optogenetic defibrillation method.

5) In chapter five, the temporal modulation of the excitability of the system is studied 
in order to control the dynamics of the arrhythmia. One of the questions addressed in this chapter is: I) How can one have better control over the dynamics of the system by applying periodic temporal modulation of its excitability? For this, I investigate dynamical control over spiral waves by applying global periodic sub-threshold illumination. At frequencies approaching those of a rotating spiral wave, the application of a sequence of illumination pulses can induce resonant drift. However, these optical pulses affect the frequency of the spiral wave, resulting in non-resonant excitation of the spiral wave. I use a frequency-based feedback algorithm to exert adaptive control over the dynamics of the spiral wave.

The second question that I address in this chapter is: II) Does feedback pacing have the potential to be considered as a low-energy defibrillation technique? For this, I examine the control of arrhythmia dynamics using the feedback method from sub-threshold to supra-threshold illumination. I then compare the efficiency of termination with feedback pacing and non-feedback pacing.

6) In chapter six, using a model for neonatal mouse ventricular tissue, I numerically show that the application of a sequence of uniform, global optical stimulation with light of high sub-threshold intensity and reasonably long pulse lengths can cause an otherwise stable spiral wave to develop wave breaks.

7) Discussion and Conclusion. The final chapter gives an overview of my key results and put them in context with the research questions that I address with my thesis. 


\section{Chapter 2}

\section{Methods}

In this chapter, I take a look at the electrophysiological properties of cardiomyocytes. I give a short overview of how these properties can be described mathematically from the level of a single cell to spatially extended cardiac tissue. I describe one particular mathematical model that is most important for my numerical studies, and go on to describe the different analytical methods that I have used in my studies. I conclude with some preliminary results.

\section{$2.1 \quad$ Background}

\subsubsection{Cardiac action potential}

Heart muscle is mainly composed of cardiomyocytes [24, 109]. These are contractile cells that enable the heart to pump, when triggered by electrical stimulation produced by the sino-atrial nodal cells (SAN) in the normal heart [25, 29]. Typically, the application of an electrical stimulus above the excitation threshold produces a characteristic response of membrane voltage called an action potential (AP) $[127,128]$. Once excited, cardiomyocytes cannot be further re-excited before the passage of a certain period of time, the so-called refractory period [33].

During sinus rhythm, an AP is formed from a group of specialized cells called the pacemaker, or SAN, located at the top of the right atrium [27]. Fig.2.1-A shows a scheme of this node. This AP is propagated through the atria and down to the ventricles to ensure coordinated contraction of all four chambers of the heart. The AP of a pacemaker and that of a contractile cell are shown in Figs.2.1-B and C, respectively. The difference in the morphology of the AP for each cell type is due to the different involvement of the ionic currents. In a pacemaker cell, as shown in Fig.2.1-B, the AP has three phases: Resting state (phase 4), depolarization state (phase 0) and repolarization state (phase 3 ). In each phase, certain types of ionic currents flow through the cell membrane. The release of sodium $\left(\mathrm{Na}^{+}\right)$ions into the cell during phase 4 leads to a gradual increase in the membrane voltage. This leads to the excitation threshold being exceeded and to the triggering of an AP during phase 0 , in which 
Methods

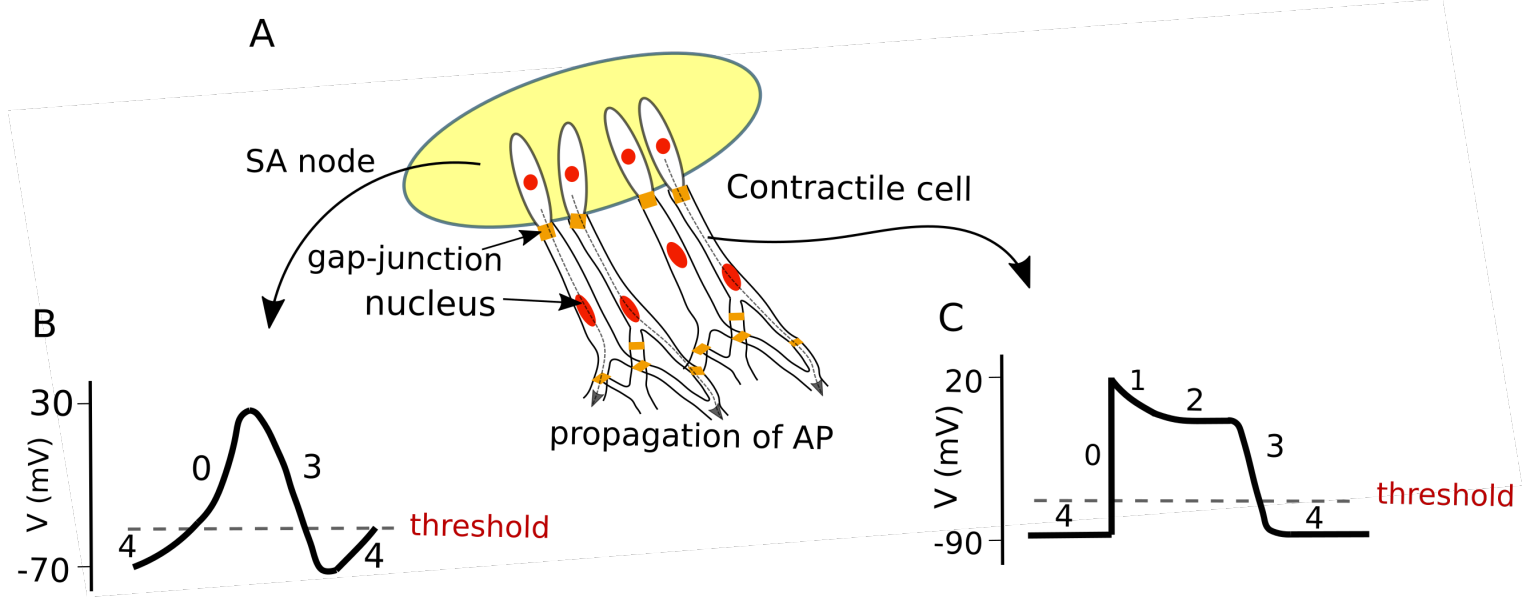

Figure 2.1 - AP propagation in a pacemaker cell and a contractile cell. A) A schematic representation of the SA node. The pacemaker cells are coupled to the contractile cells via gap-junction. B) AP of a pacemaker cell in which gradual leakage of the $\mathrm{Na}^{+}$during phase 4 triggers the automatic excitation of the cell without external stimulation. $C$ ) AP of a contractile cell showing four different phases. In each phase, certain types of ionic currents are involved. Phase 4 of this type of cell shows the resting state requires an external force to be excited.

the cell is depolarized. Thereafter, during phase 3 , the activation of the potassium channels causes a $\mathrm{K}^{+}$flow to the outside and a decrease in membrane voltage, which leads to a repolarization of the cell. Then, the membrane voltage decreases further to reach the rest state (phase 4 ) where the $\mathrm{Na}^{+}$flow gradually leaks and causes the next cycle of automatic AP triggering [113, 129, 130].

Unlike the pacemaker cell, the AP of a contractile cell has five phases, illustrated in Fig.2.1-C. During phase 4 (resting state) the net flow is zero and $I_{K 1}$ is the main potassium channel which holds the membrane voltage constant, around $-90 \mathrm{mV}$. At this phase, the contractile cell is in the diastolic phase where it is ready to be triggered. A sufficient external stimulation, exceeding the excitation threshold, causes a rapid and positive change of membrane voltage which results in cell depolarization. This abrupt activity arises from the fast activation of the $\mathrm{Na}^{+}$channels which rapidly deactivated and close. Then activation of the potassium channels $\left(\mathrm{I}_{K t o f}\right)$ allows a brief outflow of the potassium ions $\left(\mathrm{I}_{K t o f}\right)$ which causes the membrane voltage to shift slightly to more negative values and creates a notch shown as a phase 1 in Fig.2.1-C. During phase 2, there is a balance between an outward $\mathrm{K}^{+}$current known as outward delayed rectifier potassium current $\left(\mathrm{I}_{K r}\right)$, and an inward $\mathrm{Ca}^{2+}$ current from the L-type calcium channel $\left(\mathrm{I}_{\mathrm{LCa}^{2+}}\right)$. This phase is known as the plateau phase. Then the cell starts to repolarize during phase 3 . Most potassium channels now open and allow a large $\mathrm{K}^{+}$current to flow out of the cell, causing the membrane voltage to drop down to negative values, to reach the resting state (phase 4) [113, 129]. 


\subsubsection{Excitation-Contraction coupling}

In contractile cardiomyocytes, an AP is initiated by $\mathrm{Na}^{+}$channels activation triggers the L-type $\mathrm{Ca}^{2+}$ channels (as explained above) which allows an inflow of $\mathrm{Ca}^{2+}$ ions from the exterior of the cell, shown in Fig.2.2-A. This results in a process named calcium-induced calcium release where the $\mathrm{Ca}^{2+}$ coming from the L-type calcium channels bind to the ryanodine receptors2 (RyR2) and causes a release of a large amount of $\mathrm{Ca}^{2+}$ ions from the sarcoplasmic reticulum (SR), shown in Fig.2.2-A, into the cell $[131,132]$. This sudden change of $\mathrm{Ca}^{2+}$ concentration inside the cell named $\mathrm{Ca}^{2+}$ transient, is shown as a blue curve in Fig.2.2-B. Then the released $\mathrm{Ca}^{2+}$ binds to the myofilament where the cardiac contraction is initiated, the contraction signal is shown as a red dashed line in Fig.2.2-B [133-136].
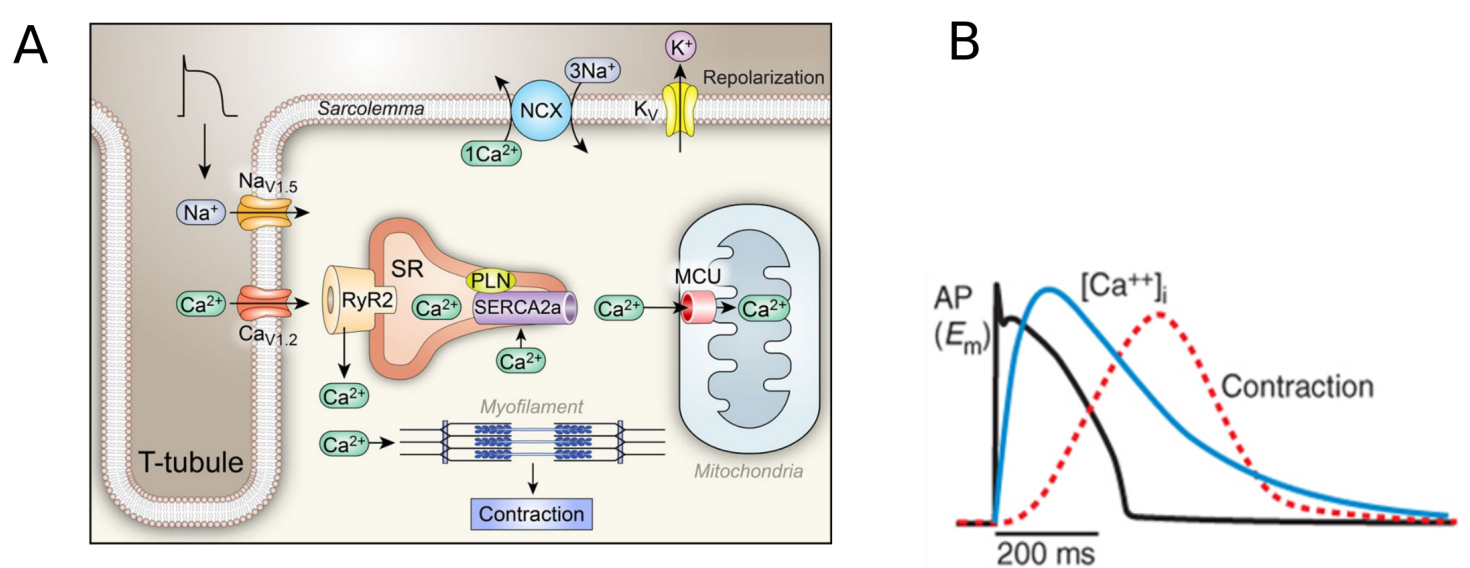

Figure 2.2 - Cell membrane and $\mathrm{Ca}^{2+}$ dynamics. A) Represents membrane and intracellular components of a cardiomyocyte. $\mathrm{Na}^{+}, \mathrm{K}^{+}, \mathrm{L}$-type $\mathrm{Ca}^{2+}$ channels, and $\mathrm{Na}^{+} / \mathrm{Ca}^{2+}$ exchanger embedded in the cell membrane. Sarcoplasmic reticulum (SR), myofilament, and mitochondria are placed inside the cell [137]. B) Shows a time series of $A P, \mathrm{Ca}^{2+}$ transient, and contraction signal measured in a rabbit heart, Taken from [135], with permission.

\subsubsection{Mathematical description of the cardiac cell membrane}

The cellular membrane is a thin lipid layer containing different types of proteins. It separates the interior of the cell from the extracellular regime [109, 111, 112]. Therefore, a membrane of a cell not only has a resistance but also has a capacitance. The electrophysiological properties of a cardiomyocyte can be represented numerically with a set of equations describing a simple electrical circuit that is shown in Fig.2.3. This circuit consists of a capacitor in parallel with a number of resistors. The capacitor represents the lipid bi-layer and each resistor represents a protein in the membrane. The amount of charge $\mathrm{Q}$ that needs to be distributed across the membrane in order to reach resting membrane voltage $\left(\mathrm{V}_{m}\right)$ with the capacitance of $\mathrm{C}_{m}$ is given by: 


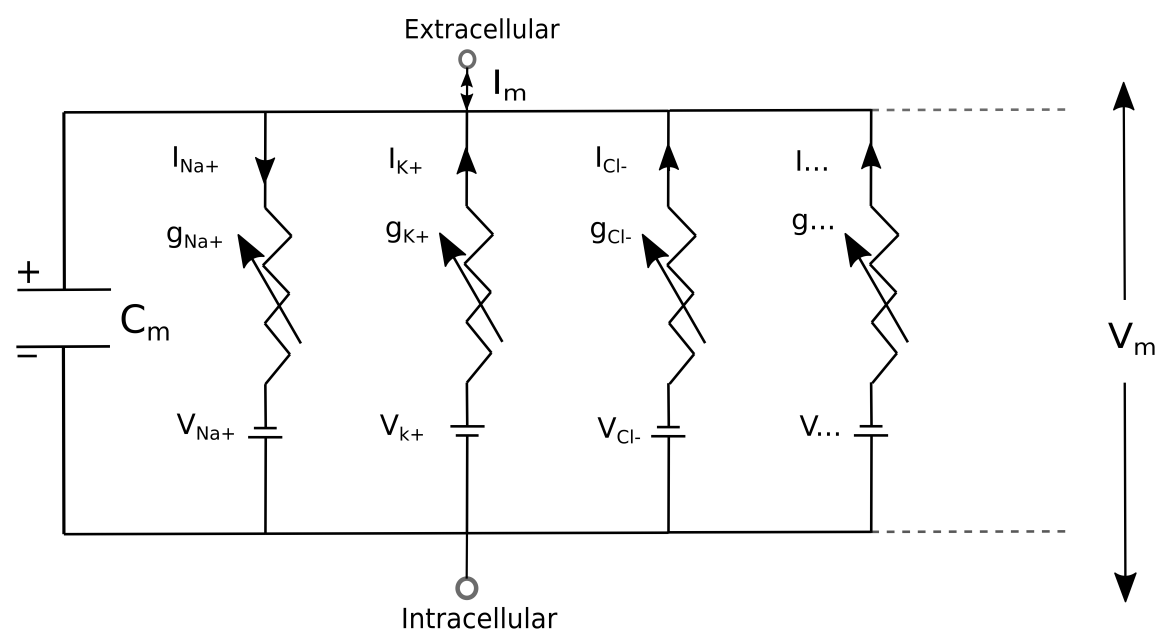

Figure 2.3 - An equivalent electrical circuit of a cell membrane. The cell membrane is shown as a capacitor charged by the membrane voltage $V_{m}$. Each ionic transporter is indicated by a resistor, showing the shows the permeability of the transporter, and the corresponding Nernst potential.

$$
Q=\frac{C_{m}}{V_{m}}
$$

Sufficient external stimulation causes a voltage gradient across the membrane. This gradient causes inflow and outflow currents through the membrane. The time evolution of the membrane voltage is described by :

$$
C_{m} \frac{d V_{m}}{d t}=I_{m}
$$

Applying Kirchhoff's current law to equation (2.2) leads to the following time derivative of the membrane voltage:

$$
C_{m} \frac{d V_{m}}{d t}=-\left(I_{N a^{+}}+I_{K^{+}}+I_{C l^{-}}+\ldots\right)
$$

\subsubsection{Cell-Cell coupling}

Individual cells of cardiac tissue are connected by a low resistance bridge named gap junction [138, 139]. An electrical excitation wave propagates and diffuses through the cardiac tissue via these junctions. Fig.2.4 shows a schematic representation of cardiomyocytes connected by gap junctions. A closer look at one of these gap junctions, shown in a dashed rectangle, indicates the involvement of several proteins called Connexons, which organize to form the pores or gaps [140, 141].

These junctions have a certain intracellular $\left(\mathrm{r}_{i}\right)$ and extracellular $\left(\mathrm{r}_{e}\right)$ resistance as shown in the equivalent electrical circuit of the cell-cell coupling in Fig.2.5. In most 


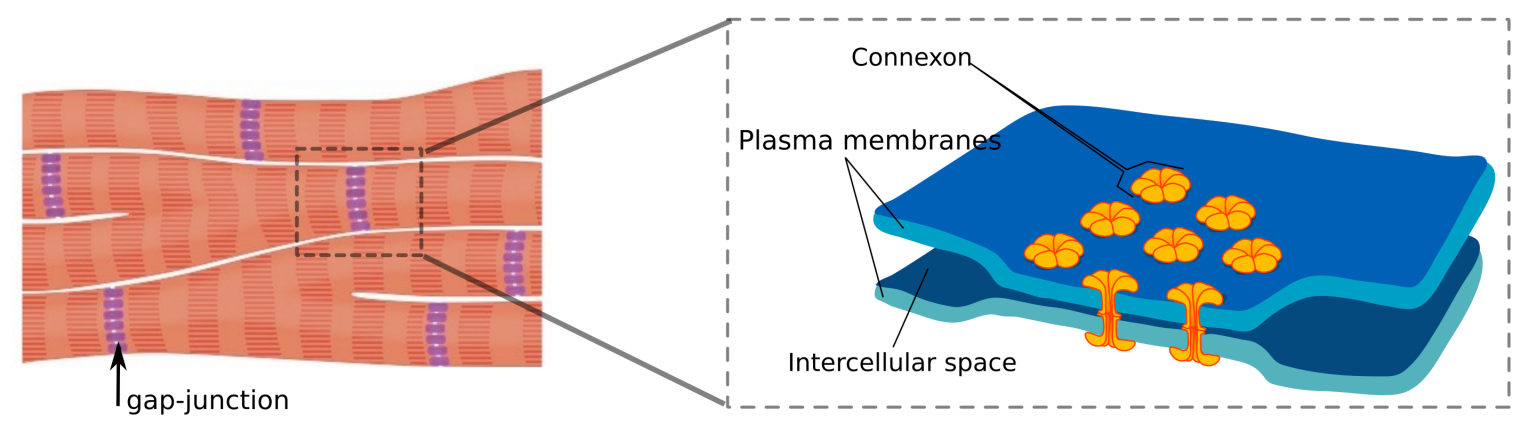

Figure 2.4 - Schematic representation of the cardiomyocytes and gap-junctions. A) Gap-junctions shown in purple connect two adjacent cells and allow ions and electrical pulses to travel through them. B) Shows a closer look at the gap-junction with some channels named Connexon form the pore for these gaps. Taken from [142], Creative Commons, CC BY 4.0.

mathematical models it is assumed that cardiac tissue consists of myocytes only [143]. One prevalent approximation to describe cardiac tissue mathematically is a continuum model where the conductivity is assumed to be either uniform or smoothly changing [143]. The current models are divided into two main categories based on the bidomain model, and the monodomain model [72, 144-147]. The former one describes current flow through both intracellular and extracellular regions of cardiac tissue. However, the latter is the simplest model that describes the current flow through the intracellular region of cardiac tissue. In this study, I use mainly the monodomain model to describe cardiac tissue. The general form of this model is:

$$
C_{m} \frac{d V_{m}}{d t}=\nabla \cdot\left(D \nabla V_{m}\right)-I_{i o n}
$$

Here $C_{m}$ is the membrane capacitance, $V_{m}$ is the membrane voltage, $\nabla$ is the gradient operator, $D$ is a diffusion matrix which for isotropic tissue is a scalar quantity.

\subsection{Modelling Cardiac optogenetics}

The electrophysiological properties of excitable cells such as neurons and cardiomyocytes can be described numerically using a simple generic model such as the Barkley model or the FitzHugh Nagumo model [148-150] or using a complex ionic model, such as the Ten-Tusscher model for human ventricular cardiomyocytes and the Luo-Rudy model for guinea pig ventricular cells [127, 143, 151-153]. Progress in the development of experimental techniques such as the patch-clamp leads to a better and more detailed description of the cell membrane [154-157].

In 1952 Hodgkin and Huxley proposed the first mathematical model for the giant squid axon. Using patch-clamp technique, they investigated two different types of ionic currents that contributed to the action potential. These currents were $\mathrm{Na}^{+}$and $\mathrm{K}^{+}$ based [154, 158]. For this discovery, they were awarded the Nobel Prize in 1963 in 


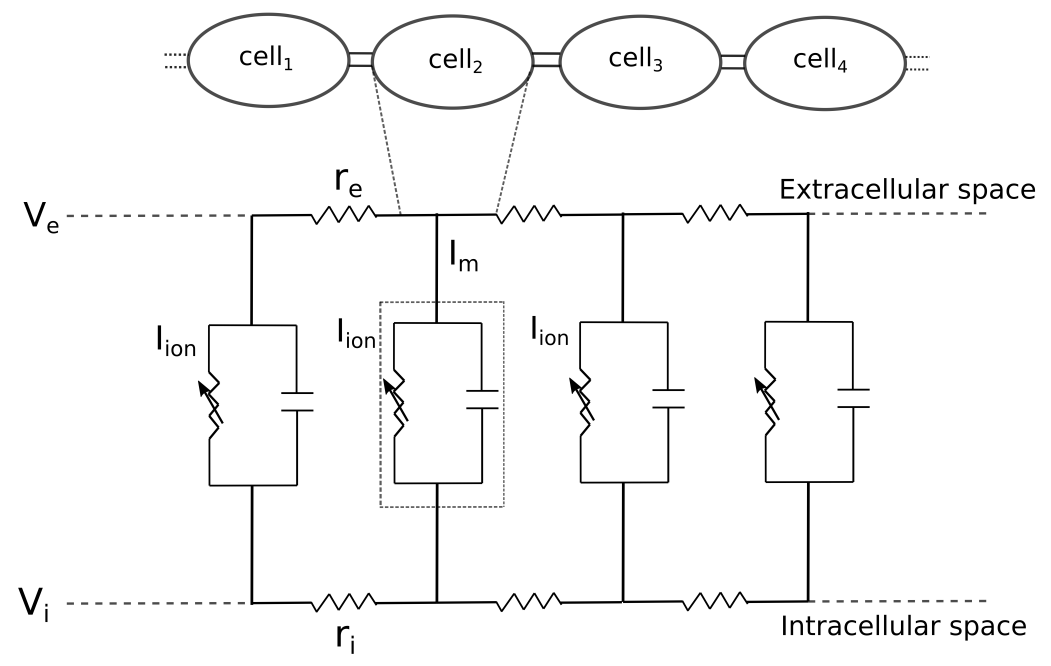

Figure 2.5 - An equivalent electrical circuit of the cell-cell coupling. The cells are connected via gap-junctions with the series of the intracellular and extracellular resistance of $r_{i}$ and $r_{e}$, respectively.

the field of medicine and biology. Following this success, Denis Noble, in 1962 [159], described the first mathematical model of a cardiac cell by adapting the equations of the Hodgkin-Huxley model according to the experimental data of the cardiac cell [160].

Following Noble's cardiac model many ionic models describing the complex interaction of multiple nonlinear time-dependent currents of a cardiac membrane were developed [143, 161-165]. These advanced models are important tools for understanding and studying the electrophysiological properties of a complex system such as the heart at different cell, tissue and organ levels [166-168].

\section{Bondarenko model: an ionic model of adult mouse ventricular cardiomyocyte}

In this study, I use a mathematical ionic model describing the cellular action potential of the ventricular adult mouse heart introduced by Bondarenko et al. [169]. This model contains of 40 dynamical variables, the time evolution of which is given by 40 ordinary differential equations (ODEs). This model has seven distinct $K^{+}$currents which are the rapidly recovering transient outward $K^{+}$current $\left(I_{K t o f}\right)$, the slowly recovering transient outward $K^{+}$current $\left(I_{K t o s}\right)$, the rapid delayed rectifier $K^{+}$current $\left(I_{K r}\right)$, the ultrarapidly activation delayed rectifier $K^{+}$current $\left(I_{K u r}\right)$, the noninactivating steadystate voltage-activated $K^{+}$current $\left(I_{K s s}\right)$, the time-independent inwardly rectifying $K^{+}$current $\left(I_{K 1}\right)$, and the slow delayed rectifier $K^{+}$current $\left(I_{K s}\right)$. Other important currents are the fast $\mathrm{Na}^{+}$current $\left(I_{\mathrm{Na}^{+}}\right)$, the L-type $\mathrm{Ca}^{2+}$ current $\left(I_{\mathrm{Ca}}\right), \mathrm{Na}^{+} / \mathrm{Ca}^{2+}$ exchange current $\left(I_{\mathrm{NaCa}}\right)$, the $\mathrm{Ca}^{2+}$ pump current $\left(I_{\mathrm{Ca}}\right)$, the $\mathrm{Na}^{+} / \mathrm{K}^{+}$pump current $\left(I_{\mathrm{NaK}}\right)$, the $\mathrm{Ca}^{2+}$ activated $\mathrm{Cl}^{-}$current $\left(I_{\mathrm{CaCl}}\right)$, the background $\mathrm{Na}^{+}$current $\left(\mathrm{I}_{\mathrm{Nab}}\right)$ and the background $\mathrm{Ca}^{2+}$ current $\left(I_{C a b}\right)$. These current make up the right-hand side 
of equation (2.5), leading to the differential equation determines the cellular action potential of this model:

$$
\begin{gathered}
-C_{m} \frac{d V}{d t}=\Sigma I_{\text {ions }}+I_{\text {stim }} \\
\Sigma I_{\text {ions }}=I_{K t o f}+I_{K t o s}+I_{K r}+I_{K u r}+I_{K s s}+I_{K 1}+I_{K s}+I_{N a^{+}} \\
+I_{C a}+I_{N a C a}+I_{C a}+I_{N a K}+I_{C a C l}+I_{N a b}+I_{C a b}
\end{gathered}
$$

Where $C_{m}$ is the membrane capacitance, $\Sigma I_{\text {ions }}$ is the summation of all involved currents, and $I_{\text {stim }}$ is an external current to stimulate the cell.

\section{Williams model: a light-responsive ionic model for ChR2}

Cardiac tissue is not light-responsive. However, expressing Opsins such as ChR2 protein in the cardiac cell membrane by external means can result in depolarization of the membrane in response to a blue light [114-117]. At negative membrane potential, ChR2 provides inward current of cations with the selectivity order of $\mathrm{H}^{+}>\mathrm{Na}^{+}>$ $\mathrm{K}^{+}>\mathrm{Ca}^{2+} \ldots$ and a reversal potential near $0 \mathrm{mV}$. The first mathematical model of ChR2 were described in neurons in response to optical stimulation [170-174]. In this study, I use a mathematical model of ChR2 which describes the function of ChR2 in a cardiomyocyte [175]. This model also describes the voltage dependency of this channel in contrast to the other existing models [124, 176-178]. The current flowing through this channel $I_{C h R 2}$ is described by the following equation:

$$
\begin{gathered}
I_{C h R 2}=g_{C h R 2} G(V)\left(O_{1}+\gamma O_{2}\right)\left(V-E_{C h R 2}\right) \\
G(V)=\frac{\left[\left(10.6408-14.6408 * \exp \left(\frac{-V}{42.7671}\right)\right)\right]}{V}
\end{gathered}
$$

Where $g_{C h R 2}$ is the maximum conductance, $G(V)$ is the voltage-dependent rectification function as it is described in equation (2.8). $O_{1}$ and $O_{2}$ are the conductances, $\gamma$ is the ratio of the conductance $O_{2} / O_{1}, V$ is the membrane voltage and $E_{C h R 2}$ is the reversal potential which is $0 \mathrm{mV}$.

Fig.2.6-A shows the $I_{C h R 2}$ at different light intensities (LIs) with a pulse length (PL) of $500 \mathrm{~ms}$ and with a constant $V$ of $-80 \mathrm{mV}$ in equation (2.7). An increase of the LI causes an increase of $I_{C h R 2}$. All cases at different LIs show the kinetics of $I_{C h R 2}$ has three main phases: 1) an initial peak, 2) a steady state, and 3) a decay to the baseline when the light is turned off $[171,174]$. This channel is not only light sensitive but also voltage sensitive [175]. Fig.2.6-B shows $I_{C h R 2}$ at different constant voltages, $V$, when a LI of $5.5 \mathrm{~mW} / \mathrm{mm}^{2}$ with a PL of $500 \mathrm{~ms}$ is applied. Due to the attraction between the negative ions inside the cell and the cations migrating through the channel inside the cell, there is a higher $I_{C h R 2}$ at lower voltage. Therefore, an increase in voltage leads to a lower $I_{C h R 2}$. 

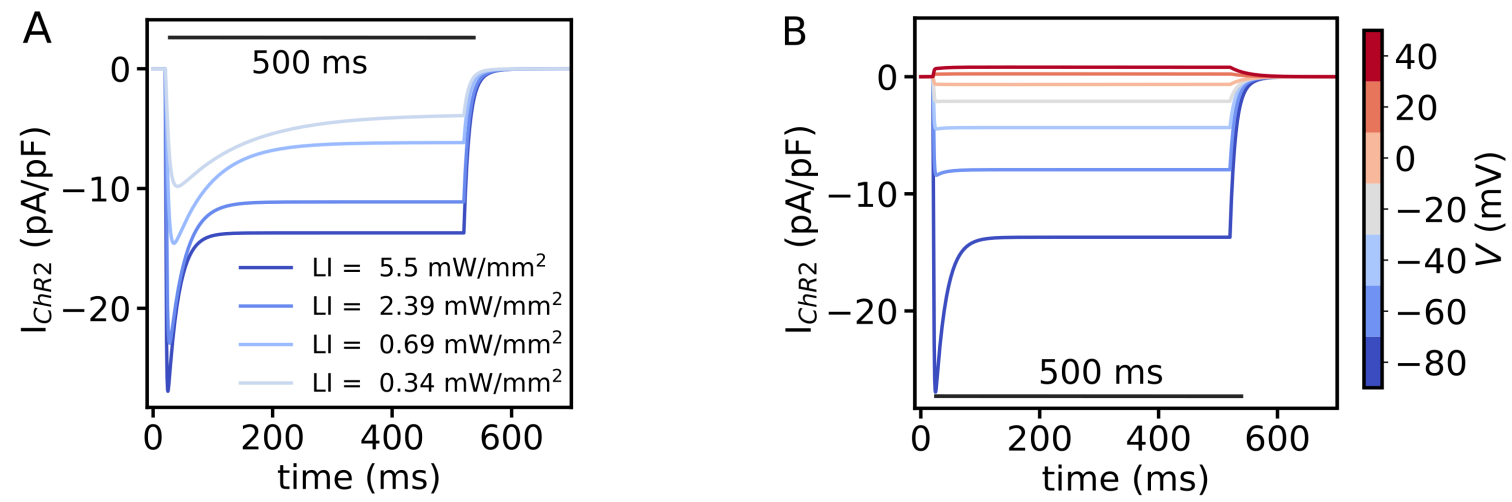

Figure 2.6 - Kinetics of ChR2. A) indicates $I_{C h R 2}$ at different LIs and PL of $500 \mathrm{~ms}$. An increase in LI causes an increase of $I_{C h R 2}$. It shows an initial peak of the $I_{C h R 2}$ which decays to a steady state and when the light is switched off it reaches the baseline. B) shows the voltage-sensitivity of the ChR2, so that increasing membrane voltage results in lower $I_{C h R 2}$.

In this study, to incorporate light-sensitivity to an ionic cell model I appended the differential equations describing ChR2 to the base model for the cardiomyocyte. I set the $\mathrm{I}_{C h R 2}$ as an $\mathrm{I}_{\text {stim }}$ as following:

$$
-C_{m} \frac{d V}{d t}=\Sigma I_{\text {ions }}+I_{C h R 2}
$$

The incorporation of the mathematical model of the ChR2 to an ionic cell model leads to the optical control of the cell function as well as electrical control. Fig.2.7-A shows a schematic representation of the bondarenko model coupled with ChR2 shown in blue. Supra-threshold electrical or optical stimulation causes the membrane voltage to exceed the excitation threshold and the cell is excited. Fig.2.7-B and C show AP of this cell model which is triggered by electrical and optical stimulation, respectively. Due to the slow kinetics of the ChR2, optical stimulation induces excitation after a certain delay time compared with the electrical stimulation.

To extend this single cell model to two-dimensions (2D) and represent the heart tissue of the ventricular adult mouse heart numerically, the diffusion term with the diffusion coefficient $\mathrm{D}$ was addedto the time derivative of the membrane voltage as follows:

$$
\frac{d V}{d t}=-\frac{I_{i o n}+I_{\text {stim }}}{C_{m}}+\nabla \cdot(D \nabla V)
$$

Here, I assume $D$ with a scalar value to have a homogeneous continuum system. I set $D$ to the value of $0.00014 \mathrm{~cm} / \mathrm{ms}$ to obtain a wave propagation with an isotropic conduction velocity $(\mathrm{CV})$ of $43.9 \mathrm{~cm} / \mathrm{s}$ which is in the range of $\mathrm{CV}$ in the mouse heart [179]. 
A

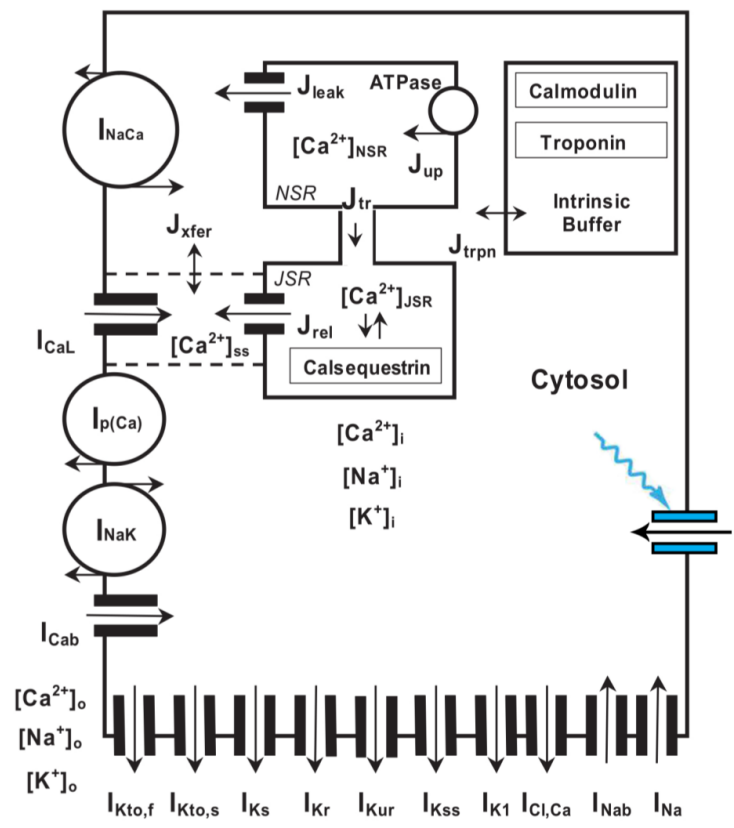

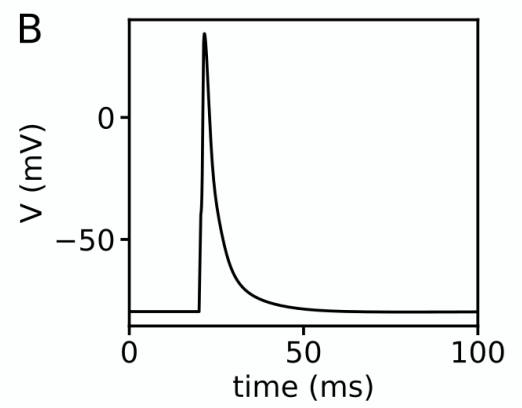

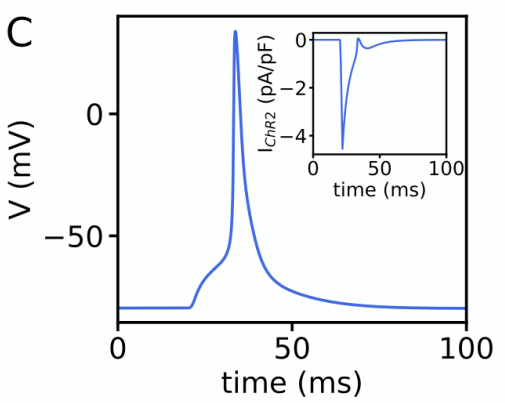

Figure 2.7 - A light-responsive cell model. A) A schematic representation of a ventricular cell model in the Bondarenko model, expressed with ChR2. B) The AP of the single cell induced by electrical stimulation. C) The AP of the single cell induced optical stimulation (blue curve). The inset shows the $I_{C h R 2}$.

\subsubsection{Spiral wave induction}

To induce a spiral wave in a 2D domain of the model, I have divided the domain into four parts. I initialized electrophysiological parameters in each part of the domain with values corresponding to four different phases of a cell membrane AP, as shown in Fig.2.8-A. Part 1 is initialized with a value when the cell is at rest, i.e. $-76 \mathrm{mV}$. Part 2 is initialized with a value of the depolarization state, i.e. $10 \mathrm{mV}$. Part 3 and part 4 are initialized with the values of the repolarization state at the beginning $(0 \mathrm{mV})$ or at the end $(-60 \mathrm{mV})$ of this state, shown in Fig.2.8-B.

During the simulation time the excitation wave at part 2 starts to propagate in the direction of the excitable region, part 1, due to the refractory state of parts 3 and 4 . After a while, part 4 reaches the resting state and is ready to be excited. This leads to a continuous propagation of the wave in the direction of this region. After a longer time part 3 reaches the resting state and is excitable so that a clear spiral is former that persists over time, Fig.2.8 D.

\subsubsection{Detecting the phase singularity of a spiral wave}

In periodic dynamics, it is useful to represent the state of each element by its phase

$(\theta)$ [180]. Propagation of the excitation waves in cardiac tissue causes each excitable 
Methods
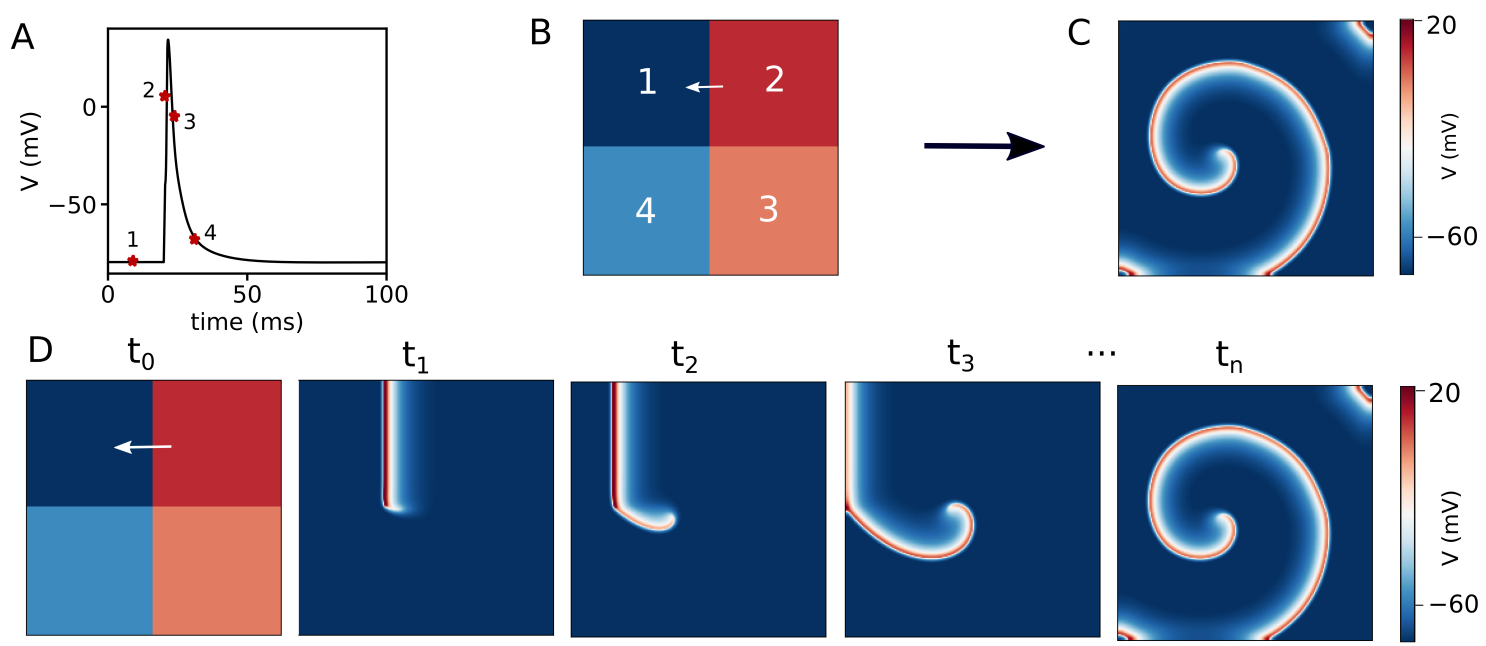

Figure 2.8 - Spiral wave induction. A) AP of a single cell for the ventricular mouse heart. Four different values of the $A P$ are shown with a red star: during the resting state (1), excitation (2), early refractory period (3) and late refractory period (4). B) Initialization of the domain with a size of $200 \times 200$ grid point with four different values given in (A). C) Formation of a spiral wave in the domain. D) A series of snapshots show the formation of the spiral wave

component in heart tissue to undergo a phase change in the range of $-\pi$ to $+\pi$ [181]. For the case of rotating spiral waves, there is one point, the so-called phase singularity, which has no specific phase and is the organizing center of the rotating wave [180]. These centers play an important role in the functioning of wave and the persistence of cardiac arrhythmias [182]. The detection of the location of these singularities might help to have better control over the dynamics of the spiral waves $[180,182]$. One method to detect the phase singularity is phase mapping over the entire heart tissue during an arrhythmia. This involves calculating the phase of all excitable components of the tissue over time. This results in a phase map $(\theta(\mathrm{x}, \mathrm{y}, \mathrm{t}))$ that shows a unique spatial pattern near the tip of the spiral waves, with the phase of all adjacent components in this region changing from $-\pi$ to $+\pi$ around the center, corresponding to a phase singularity [183]. For the simple two or three variable models of cardiac cell, the phase angularity can be calculated from the excitory and refractory variables by the following function:

$$
\theta(t)=\arctan 2\left[u(x, t)-u^{*}, v(x, t)-v^{*}\right]
$$

Where $u$ is the excitable and $v$ the refractory variable, $u^{*}$ and $v^{*}$ is a point in the local phase space which was surrounded by the trajectory during an action potential. Figs.2.9 A-C shows a snapshot of a spiral wave in the distribution of the $u$ variable, $v$ variable, and the phase $\theta$, respectively.

To calculate the phase singularity of more complex ionic models or experimental data a reconstructed phase space is required [184, 185]. For this purpose, I use time 

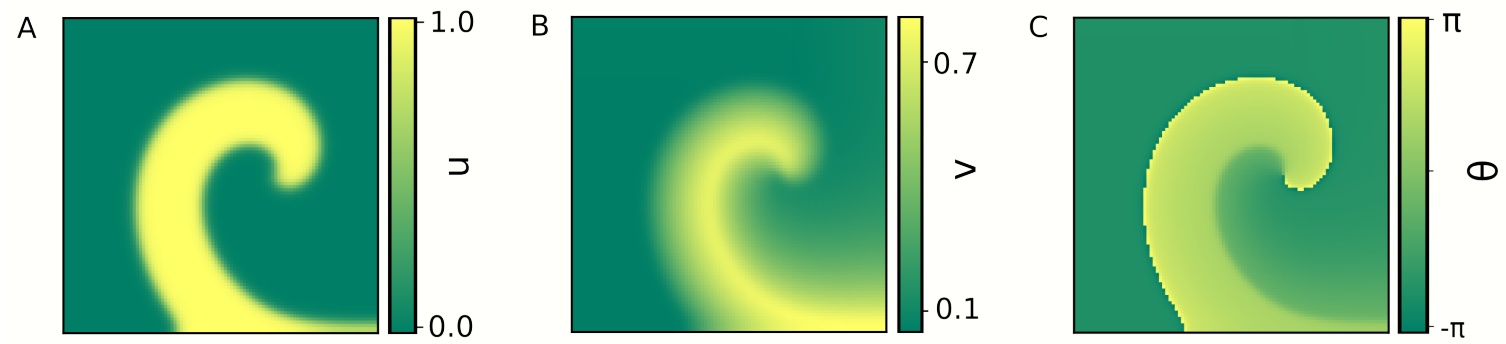

Figure 2.9 - Propagation of a spiral wave in the Barkley model in the distribution of A) the $u$-variable, $B$ ) the v-variable and c) the phase $\theta$.

series of the voltage variable and apply the technique of time delay embedding [184]. The phase singularity is calculated as follows:

$$
\theta(t)=\arctan 2\left[V(t+\tau)-V^{*}, V(t)-V^{*}\right]
$$

The phase space is obtained by plotting $V(t)$ against $V(t+\tau)$ of a rotating spiral wave with a period of $64 \mathrm{~ms}$, as shown in Fig.2.10. The time delay $(\tau)$ is usually defined as half a period of the arrhythmia.

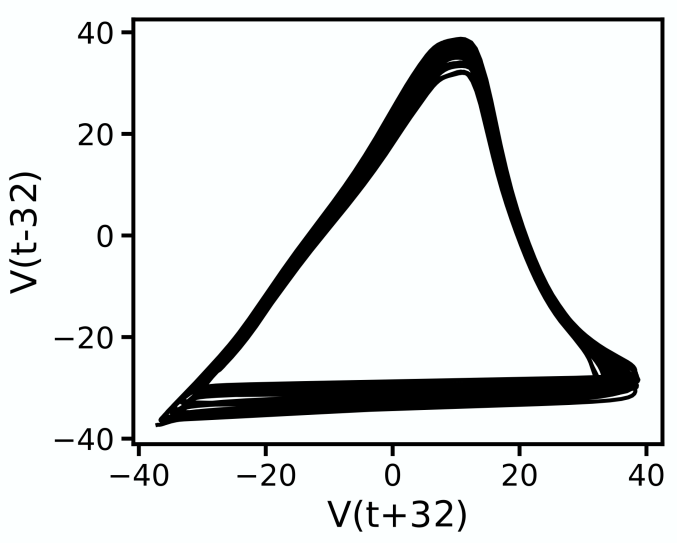

Figure 2.10 - Reconstructed phase space of a spiral wave using voltage variable. The delay time, $\tau$ is $32 \mathrm{~ms}$ for the spiral wave with the period of $64 \mathrm{~ms}$.

\subsubsection{Determining the optical excitation threshold}

In this study, I control the spiral wave dynamics by applying different LIs from sub to supra-threshold illumination. To know which intensities are sub or supra-threshold we need to know what the excitation threshold is. For this, I applied global illumination in a quiescent $2 \mathrm{D}$ domain with varying $\mathrm{LI}$ and PL. Then, I checked the activity of a point in the domain at each PL with a corresponding LI whether there is an excitation activity (supra-threshold) or not (sub-threshold), where, the membrane voltage is elevated below the threshold. Fig.2.11-A shows a dose-response curve at different LIs and PLs. It illustrates the excitation threshold of $0.02 \mathrm{~mW} / \mathrm{mm}^{2}$. Fig.2.11-B shows 
the average of membrane voltage of the domain after $1000 \mathrm{~ms}$ of a constant global illumination with $\mathrm{LI} \leqslant 0.02 \mathrm{~mW} / \mathrm{mm}^{2}$.
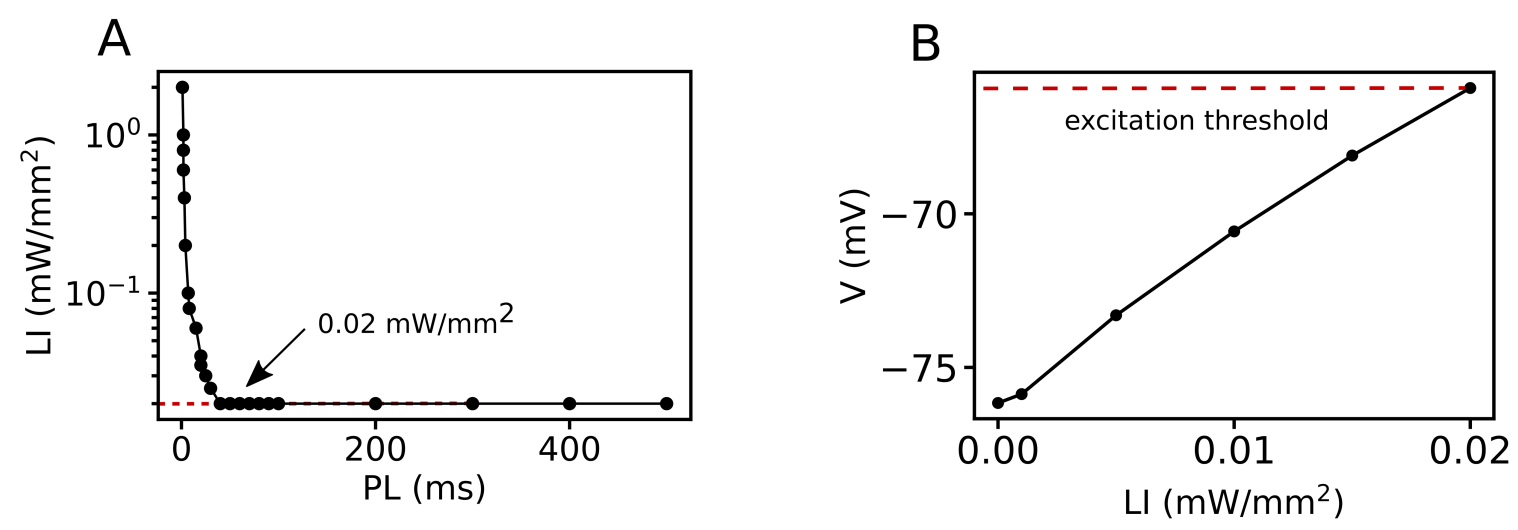

Figure 2.11 - Rheobase study and excitation threshold. A) Shows the rheobase curve, which indicates the lowest $L I$ that can excite the domain with infinite PL. B) Shows the effect of a globally uniform illumination below the threshold on the membrane voltage of a $2 D$ domain. It illustrates the increase in $L I$, which increases the membrane voltage.

\subsubsection{Calculating curvature of a spiral wave's tip trajectory}

In this work, the spiral wave's tip rotates naturally in a circular path with a constant curvature. The application of periodic illumination or constant illumination patterns causes different trajectory of the tip which results in a change of curvature. For curves, the curvature at a particular point on the curve is shown by a circle that best fits the curve near that point, as shown in Fig.2.12. The curvature $(k)$ is equal to the reciprocal of the circle radius $(r)$ :

$$
k=\frac{1}{r}
$$

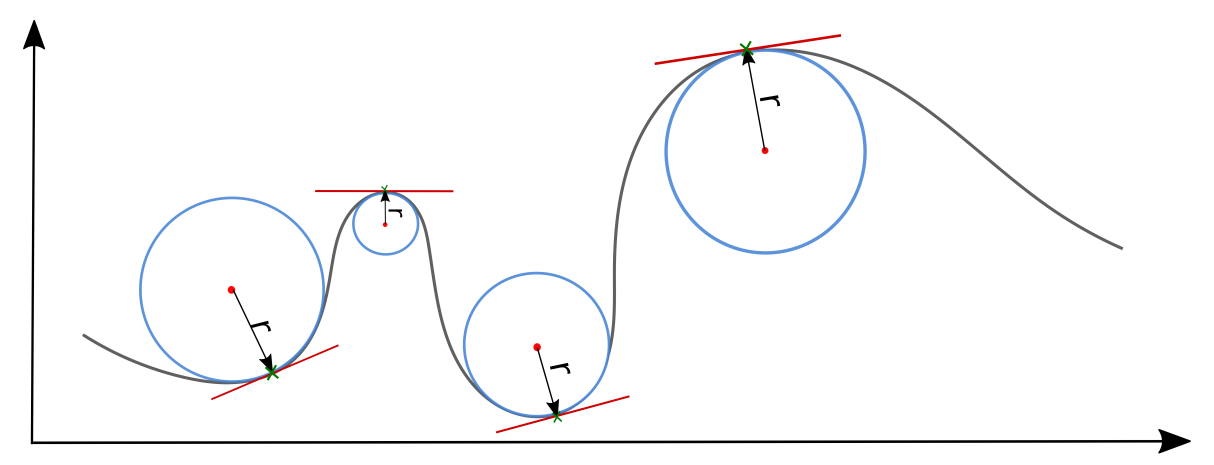

Figure 2.12 - A 2 dimensional line with different values of curvature. The corresponding circle for a particular point on the curve, shown with a green star, indicates that the radius of the circle is larger at low curvature and vice versa. 
To measure the instantaneous curvature of each point of the trajectory of the spiral wave's tip with a coordinate of $(x, y)$, I have used the following formula:

$$
k=\frac{x^{\prime} y^{\prime \prime}-y^{\prime} x^{\prime \prime}}{\left(x^{\prime 2}+y^{\prime 2}\right)^{\frac{3}{2}}}
$$

Here $x^{\prime}$ and $y^{\prime}$ are the first derivative of the coordinate $(x, y)$ with respect to time and $x^{\prime \prime}$ and $y^{\prime \prime}$ are the second derivative of the coordinate $(x, y)$ with respect to time.

\subsection{Experimental methods}

To study the effect of global low LI illumination on arrhythmia, I used an experimental setup developed in our group for an intact ChR2 heart of an adult mouse [23]. This setup consists of a perfusion bath with hexagonal geometry surrounded by three LEDs (wavelength $470 \mathrm{~nm}$ ) to apply a globally uniform illumination. Fig.2.13 shows a sketch of such an experimental setup. To perform an optical mapping experiment, a camera (Cascade 128+, Photometrics) with a mounted LED with the wavelength $625 \mathrm{~nm}$ was installed on the right side of the bath.

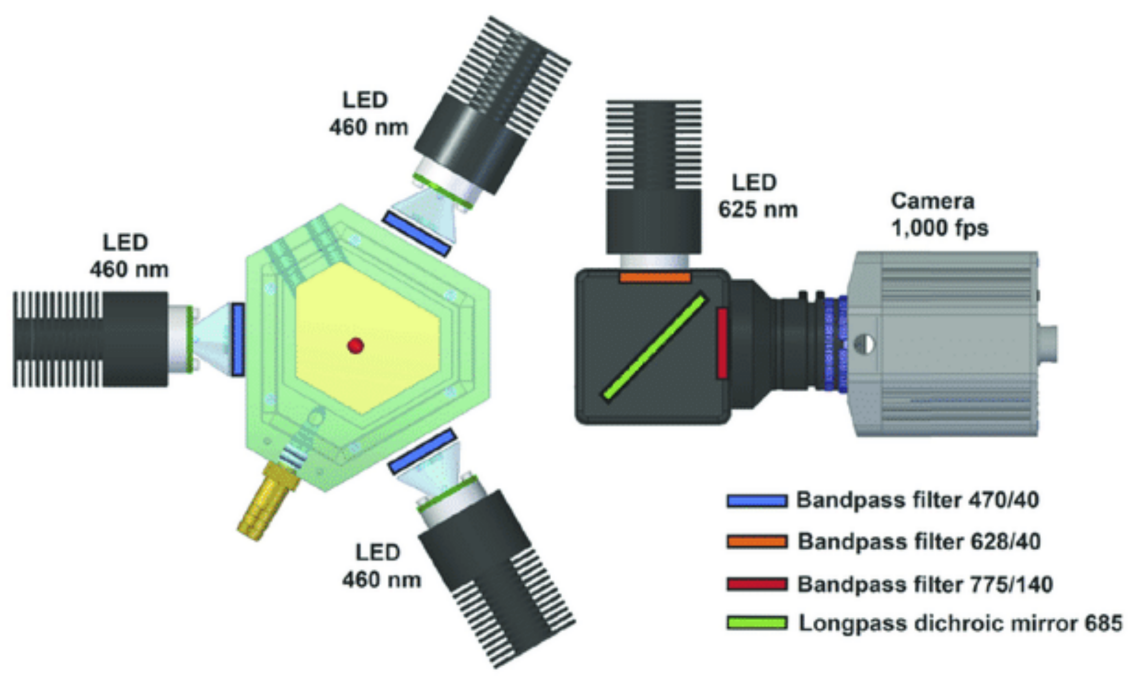

Figure 2.13 - Sketch of the experimental setup of the global illumination. The hexagonal shape is the bathtub with a heart inside, which is represented by a red dot. This bathtub is surrounded by three LEDs. On the right side of the path there is a camera with an LED for optical mapping. Taken from [23], an open access article.

After the extraction of the transgenic heart from the mouse, the heart is perfused with normal Tyrode solution for about 20 minutes. This solution preserves the electrophysiological conditions of the heart and contains $30 \mathrm{mM} \mathrm{NaCl}, 4 \mathrm{mM} \mathrm{KCl}, 1$ $\mathrm{mM} \mathrm{MgCl} 2,24 \mathrm{mM} \mathrm{NaHCO}_{3}, 1.8 \mathrm{mM} \mathrm{CaCl}_{2}, 1.2 \mathrm{mM} \mathrm{KH}_{2} \mathrm{PO}_{4}, 5.6 \mathrm{mM}$ glucose, $1 \%$ albumin/BSA were aerated with Carbogen (95\% oxygen and $\left.5 \% \mathrm{CO}_{2}\right)$ [186]. To induce 
a persistent ventricular arrhythmia, we used a low potassium normal Tyrode, the $\mathrm{KCl}$ concentration was reduced from $4 \mathrm{mM}$ (normal styrene) to $2 \mathrm{mM}$, with a combination of $100 \mu \mathrm{M}$ Pinacidil to shorten APD [187, 188]. We let the heart perfuse with the arrhythmia induction solution for about 20 minutes. Then, I induced a persistent arrhythmia in the ventricular part with electrical overdrive stimulation (frequency of $30-35 \mathrm{~Hz}$ and pulse width of 6-7 ms).

For the experimental studies of global periodic stimulation to control cardiac arrhythmias in an intact mouse heart, an electronic device was developed to perform periodic stimulation at a constant phase of the arrhythmia, named feedback pacing. This device detects a critical voltage using an electrode located on the surface of the heart. Then it sends a signal to the signal generator device, which triggers a sequence of global illumination pulses with a frequency close to the arrhythmia frequency. 


\section{Chapter 3}

\section{Control of arrhythmia using single supra-threshold optical pulse}

\subsection{Scientific goal and findings}

In ventricular tachycardia (VT), the heart rate is faster than normal. This increased rate is attributed to the occurrence of high frequency spiral waves that override the pacemaker and serve as primary drivers of cardiac electrical activity. A clinical method used to control this abnormal electrical activity in the heart is anti-tachycardia pacing (ATP). It involves sending a sequence of pulses at a frequency higher than the frequency of the arrhythmia. These high frequency pulses lead to the propagation of a train of new waves which force the core of the spiral to drift towards an inexcitable boundary in favor of elimination. However, this method works only if the arrhythmia has a single dominant frequency. In the case of an irregular rhythm, as occurs in fibrillation, defibrillation is the most effective method to eliminate the abnormal electrical activity of the heart. It involves the application of a single high-voltage electric shock to the heart to control chaotic wave dynamics. However, the exact mechanisms underlying defibrillation are incompletely understood. Development of a clear understanding of these mechanisms can help in the design of new strategies for optimally terminating complex arrhythmias.

In this study, I investigate the termination of a spiral wave using a single global light pulse at different light intensities (LIs) and pulse lengths (PLs). At very low LIs, the phase singularity (PS) at the tip of the spiral wave is progressively pushed towards the domain boundary (B) to annihilate by PS-B collision. In contrast, at high LIs, abrupt termination of the spiral wave occurs because of forced phase-resetting of the domain with subsequent removal of the PS.

\section{Manuscript Status}

The manuscript is under preparation 
Control of arrhythmia using single supra-threshold optical pulse

\section{Author Contributions}

- Sayedeh Hussaini designed the research, performed all numerical simulations, analyzed the experimental and numerical data, and wrote the manuscript

- Vishalini Venkatesan conducted and analyzed the experiment

- Raul A. Quinonez Uribe designed the experimental setup for global illumination of the intact mouse heart

- Claudia Richter designed the research and edited the manuscript

- Rupamanjari Majumder designed the research and wrote the manuscript

- Stefan Luther designed the research and wrote the manuscript

\subsubsection{Manuscript}

\section{Abstract}

Ventricular fibrillation (VF), typically associated with the occurrence of chaotic electrical activity in the heart, is a leading cause of sudden cardiac death in the Western world. The most effective method used to treat fibrillation involves administering a powerful, globally resetting electrical shock to the heart to restore its normal function. This method is known as defibrillation. Unfortunately, despite its high success rate, conventional defibrillation is associated with serious side effects such as tissue damage, severe pain and trauma, motivating the need for alternative therapeutic strategies. However, the development of new painless and non-invasive treatment approaches requires a detailed understanding of the dynamics of spiral waves in cardiac tissue, especially during defibrillation. Thus, in this study, we investigate the mechanisms underlying conventional defibrillation using optogenetics. Instead of applying a single electrical shock, here, we use a single globally-illuminating optical pulse to eliminate the spiral wave from two-dimensional (2D) cardiac tissue. We subsequently compare our observations with the electric shock method.

Our results show a similar dose-response curve for both optical and electrical stimulation. An increase in the intensity and duration of the applied stimulus leads to an increase in the efficiency of arrhythmia termination. However, the mechanism responsible for effectuating termination is different in different illumination régimes. At low light intensities (LIs), spiral wave termination occurs through progressive expansion of the spiral core, with gradual movement of the phase singularity (PS) at the spiral tip towards an inexcitable boundary (B), in favor of annihilation (PS-B collision). On the other hand, at high LIs, the entire domain is excited almost synchronously, resulting in phase reset around the tip of the spiral wave, which ensures removal of the PS. Thus, in the first case, arrhythmia termination is associated with a transient time, whereas, in the second case, termination occurs almost immediately, similar to conventional electrical defibrillation. 


\section{Introduction}

Occurrence of electrical spiral waves in the heart has been known since the beginning of the 19th century. A simple spiral wave is stationary in space, rotating around a fixed point, or moving periodically around a circular core which remains excitable at all times. In this case, the tip of the spiral wave exhibits a circular trajectoryHowever, in complex excitable media such as cardiac tissue, spiral waves have the potential to exhibit rich dynamical behaviour, such as breakup, drift, and meander. Breakup is said to occur when new self-sustaining waves emerge spontaneously from a parent spiral. Drift, on the other hand, does not involve formation of new waves, but movement of the original spiral within the domain, such that, over time, the core shifts to a new location, different from the original position in space. If, however, the spiral moves within the domain such that its tip traces a closed trajectory with repeated structures, then it is said to meander [189]. Meander patterns can be linear (Z-type) or floral (hypocycloid or epicycloid, depending on the direction of the petals: outward or inward, respectively). Depending on the type of spiral wave present in the system, the approach to control its dynamical behaviour needs to be modified. Thus, understanding spiral wave dynamics has become a major goal for researchers interested in nonlinear dynamics and complex excitable systems.

In the heart, a single spiral wave in accociated with tachycardia [45]. An effective low-energy clinical method used to treat tachycardia is anti-tachycardia pacing (ATP) which is used to terminate spiral waves by inducing spatiotemporal drift [64, 65, 68]. This drift is induced by sending a sequence of electrical pulses with a frequency higher than the frequency of the spiral wave. Successive pulses penetrate close and closer in time to the core of the spiral wave, pushing the tip away from the source of external stimulation until it collides with the boundary [66, 67]. However, high-frequency arrhythmia such as ventricular fibrillation (VF) cannot be controlled with this method. In such cases, clinicians resort to applying a single high-energy shock to the heart to reset all electrical activity and restore normal cardiac function. Unfortunately, this approach has noticeable side effects such as tremendous pain, trauma and tissue, which necessitates the search for low-energy defibrillation methods [6-9]. In order to develop effective low-energy defibrillation methods that are suitable for clinical use, it is important to develop a detailed understanding of spiral wave dynamics during an arrhythmia and the mechanisms that lead to successful control.

Recent ongoing research demonstrates the applicability of optogenetics to control cardiac arrhythmia in transgenic ChR2 mouse heart by applying different patterns from structured to global illumination [22, 23, 99]. Besides, some studies show that this technique can be used as a tool to improve our understanding of the dynamics of a spiral wave $[10,36]$. Analogous to the conventional single global electric shock method, in this study, we apply a single global optical pulse to a spiral wave within a $2 \mathrm{D}$ domain of optogenetically modified cardiac tissue. We investigate the dynamics of the wave at different LIs and PLs and show how this method is comparable to the conventional defibrillation technique. Our results demonstrate similar behaviour of the termination rate at different LIs and PLs; an increase in LI or PL leads to an 
increase in the efficiency of arrhythmia termination. The underlying mechanism of termination at high LIs shows an abrupt excitation of the whole area, which prevents further propagation of the spiral wave. The part of the domain surrounding the spiral tip is forced to undergo a phase reset (along with the rest of the tissue). This results in removal of the phase singularity at the tip, and subsequent disappearance of the spiral. This mechanism is similar to conventional defibrillation. However, at low LIs, we observed a different mechanism of termination in which there is progressive growth of the spiral core. In contrast to immediate termination of the spiral wave at high LIs, there is a transient termination time at low LI.

\section{Methods}

\section{Numerical Study}

For numerical studies we used a reaction-diffusion-type equation to describe the spatiotemporal evolution of the membrane potential $(V)$ of cardiomyocytes, as shown in Eq. 3.1. The total ionic current $\left(I_{i o n}\right)$ flowing across the membrane of each cardiomyocyte was formulated using a modified version of the Bondarenko model for adult mouse ventricular cells $[169,190]$.

$$
\begin{gathered}
\frac{d V}{d t}=-\frac{I_{\text {ion }}+I_{\text {stim }}}{C_{m}} \\
I_{\text {ions }}=I_{N a^{+}}+I_{C a L}+I_{p C a}+I_{K t o f}+I_{K t o s}+I_{K r}+I_{K u r}+I_{K s s}+I_{K 1} \\
+I_{K s}+I_{N a C a}+I_{N a K}+I_{C a C l}+I_{C a b}+I_{N a b}
\end{gathered}
$$

Here, $I_{N a}$ is the fast $\mathrm{Na}^{+}$current, $I_{C a L}$ is the L-type $\mathrm{Ca}^{2+}$ current, $I_{p C a}$ is the $\mathrm{Ca}^{2+}$ pump current, $I_{K t o, f}$ is the rapidly recovering transient outward $K^{+}$current, $I_{K t o, s}$ is the slowly recovering transient outward $K^{+}$current,$I_{K r}$ is the rapid delayed rectifier $K^{+}$current, $I_{K u r}$ is the ultrarapidly activating delayed rectifier $K^{+}$current, $I_{K s s}$ is the non-inactivating steady-state voltage-activated $K^{+}$current, $I_{K 1}$ is the time-independent inwardly rectifying $K^{+}$current, $I_{K s}$ is the slow delayed rectifier $K^{+}$ current, $\mathrm{I}_{\mathrm{NaCa}}$ is the $\mathrm{Na}^{+} / \mathrm{Ca}^{2+}$ exchange current, $\mathrm{I}_{\mathrm{NaK}}$ is the $\mathrm{Na}^{+} / \mathrm{K}^{+}$pump current, $I_{C l, C a}$ is the $\mathrm{Ca}^{2+}$-activated $\mathrm{Cl}^{-}$current, $I_{\mathrm{Cab}}$ is the background $\mathrm{Ca}^{2+}$ current, and $\mathrm{I}_{\mathrm{Nab}}$ is the background $\mathrm{Na}^{+}$current.

To incorporate light-sensitivity to this ionic model, we incorporated a mathematical model for the light-responsive protein Channelrhodopsin2 (ChR2). This protein is activated with blue light $(470 \mathrm{~nm})$ and allows an inward flow of cations upon activation. The ChR2 current is described as follows:

$$
I_{C h R 2}=g_{C h R 2} G(V)\left(O_{1}+\gamma O_{2}\right)\left(V-E_{C h R 2}\right)
$$

Here $g_{C h R 2}$ is the conductance, $G(V)$ is the voltage rectification function, $O_{1}$ and $O_{2}$ are the open state probabilities of the ChR2, $\gamma$ is the ratio $O_{1} / O_{2}$, and $E_{C h R 2}$ is the reversal potential of this channel. By including the mathematical model of the ChR2 
to this model, we can stimulate the system optically at the single cell level or the 2D level.

\section{Experimental Study}

To study the dose-response curve for the rate of termination of an arrhythmia (expressed as a percentage) at different LIs and PLs, we applied a single global light pulse to Langendorff-perfused hearts extracted from $\alpha$-MHC-ChR2 transgenic mice. To induce arrhythmia, we applied 30 electrical pulses with the amplitude of 2.3-2.5 V and the frequency of 30-50 Hz using a needle electrode. To provide a global illumination a single blue light with a certain PL was applied using three LEDs located at three different sides around the bath with angular separation of $120^{\circ}$.

\section{Results}

Standard defibrillation delivers a single electrical shock of enormous field strength to the entire heart tissue. This causes the entire tissue to be excited at once, with the excitation waves preventing further propagation of the arrhythmia. As a result phase resetting occurs, which is followed by synchronized recovery from excitation throughout the heart and sinus rhythm is restored. Analogous to the conventional defibrillation method, we apply a single global optical pulse to a spiral wave rotating in a light-responsive cardiac tissue. Our goal is to investigate the mechanism(s) underlying arrhythmia termination using optogenetics. We compare our findings with the case of conventional defibrillation.

First, we induce a single spiral wave in a 2D domain $(25 \mathrm{~mm} \times 25 \mathrm{~mm})$. Fig.3.1-A shows a single spiral wave with a circular core trajectory. To investigate the excitation activity within the core, we consider the membrane voltage along the $\mathrm{x}$-axis at $\mathrm{y}$ $=12.5 \mathrm{~mm}$ passing through the core region, shown with a dashed line in Fig.3.1-A. Fig.3.1-B shows temporal evolution of the membrane voltage during $500 \mathrm{~ms}$ of spiral wave rotation along the dashed line. The red shaded rectangle illustrates the region within the core ( $0.3 \mathrm{~mm}$ wide) where the membrane voltage is locally minimum. Here, the membrane voltage increases to values that are always below the excitation threshold. Thus, no activation occurs from within the core around which the tip of the spiral rotates. Fig.3.1-C shows the space-time diagram of the membrane voltage along the dashed line. The blurred area marked with arrows (right) shows the core region of the spiral wave. The voltage time series $V(6.25 \mathrm{~mm}, 12.5 \mathrm{~mm}, t)$, corresponding to the solid white line shown in $(\mathrm{C})$, during $500 \mathrm{~ms}$ of the simulation time shows that the spiral rotates with a frequency $\approx 15.3 \mathrm{~Hz}$.

To control the dynamics of the spiral wave in favor of termination, we apply a single global optical pulse at two different LIs $\left(30 \mu \mathrm{W} / \mathrm{mm}^{2}\right.$ and $\left.1000 \mu \mathrm{W} / \mathrm{mm}^{2}\right)$ and $\mathrm{PL}=300 \mathrm{~ms}$. Fig.3.2-A shows a sequence of frames demonstrating the termination of the spiral wave with LI $=30 \mu \mathrm{W} / \mathrm{mm}^{2}$. It shows that the annihilation of the spiral wave is initiated from the core region, pushing the phase singularity (PS) at the tip towards the domain boundary (B) where it annihilates by PS-B collision. As we 

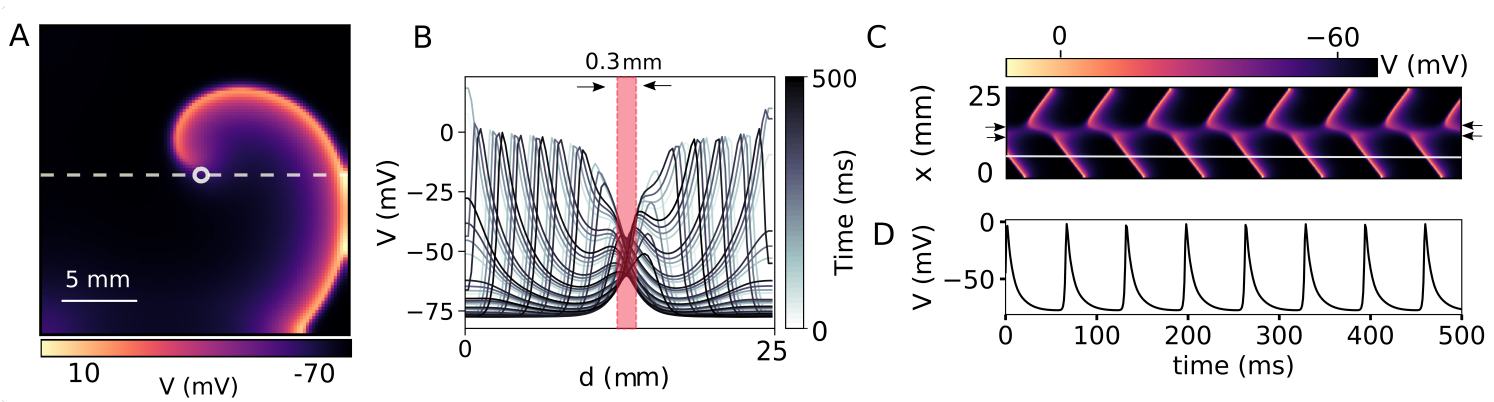

Figure 3.1 - A single spiral wave with a circular core. A) A spiral wave rotates in a $2 D$ domain $(25 \mathrm{~mm} \times 25 \mathrm{~mm}$ ) with a circular core (diameter $=0.3 \mathrm{~mm}$ ). B) Voltage distribution during $500 \mathrm{~ms}$ of simulation, across the dashed line, shown in A, which passes through the core area. The red shaded region illustrates a local minimum of the membrane voltage showing only elevation of the membrane voltage below the excitation threshold which results in no activation inside the core area. C) Space-time plot of the membrane voltage across the dashed line shown in A, during $500 \mathrm{~ms}$ of the spiral wave rotation in the domain. The blurred region indicated with arrows demonstrates the core region of the spiral wave. D) The voltage-time series $V(6.25 \mathrm{~mm}, 12.5 \mathrm{~mm}, t)$ across the solid white line in $C$.

discussed above, there is no activity in the core region. The spiral appears to dissolve from its core. Fig.3.2-B shows the voltage time series $V(6.25 \mathrm{~mm}, 12.5 \mathrm{~mm}, t)$, shown in Fig.3.2-A with a cross mark, before, during and after illumination. At the beginning of illumination, there is an oscillatory phase represented by a red dashed box. This phase shows a transient termination time of $\approx 100 \mathrm{~ms}$, which is the time difference between the onset of illumination and the last peak of the arrhythmia action potential (AP). After this, there is an elevated constant phase, shown with a green dashed box, during which the recorded point membrane voltage is in a constant depolarised state. After the light is turned off, there is a decaying phase, shown with a blue dashed box, in which the membrane voltage of the recording point drops to the resting membrane voltage, corresponding to a resting state. Fig.3.2-C shows a series of the frames showing the termination of the spiral wave during illumination with LI of $1000 \mu \mathrm{W} / \mathrm{mm}^{2}$. It shows an abrupt excitation of the whole region, which impedes further propagation of the spiral wave, ensuring phase-reset and leading to a sudden termination. The corresponding voltage time series of the recording electrode shows a very short termination transient time, less than $10 \mathrm{~ms}$. Thereafter, the system enters the constant elevated phase, which then decays to the quiescent state once the light is switched off.

We observed that at low LIs, the spiral wave terminated via progressive dissolution of its core. In contrast, at high LI, termination occurred abruptly within a short time span, which is comparable to the conventional defibrillation method. To develop a better understanding of the process of core dissolution, we apply a single global pulse to the spiral wave in a wide range of LIs from sub- to supra-threshold illumination with the PL of $500 \mathrm{~ms}$. Fig.3.3 shows a space-time plot of the membrane voltage along 

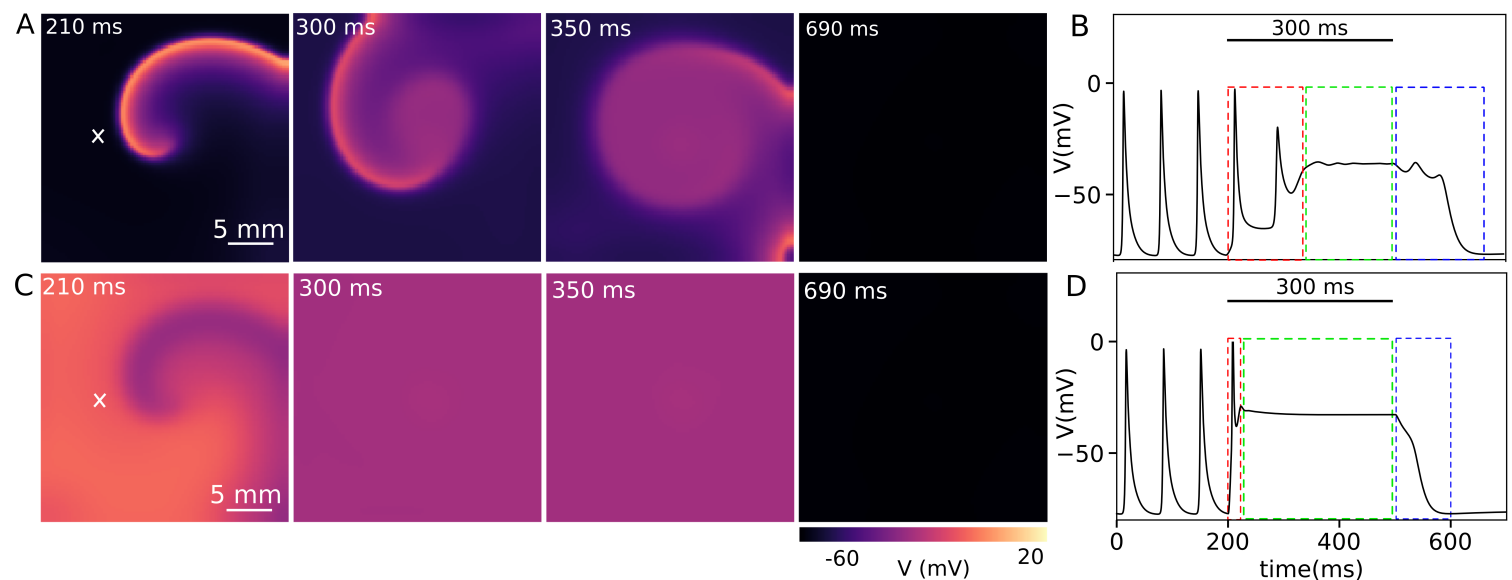

Figure 3.2 - Control of arrhythmia by applying a single global optical pulse. A) Termination of a spiral wave in a $2 D$ domain at light intensity (LI) of $30 \mu \mathrm{W} / \mathrm{mm}^{2}$ and Pulse length (PL) of $300 \mathrm{~ms}$. It shows a progressive termination starting from the core region. B) Voltage-time series of a point (shown with a cross marker) with the coordinate of $(0.625 \mathrm{~cm}, 1.25 \mathrm{~cm})$ before, during, and after illumination. Three different phases of oscillatory, elevated constant, and relaxation phase are shown with three dashed red, green, and blue boxes, respectively. C) Termination of the spiral wave with LI of 1000 $\mu \mathrm{W} / \mathrm{mm}^{2}$ with $P L$ of $300 \mathrm{~ms}$. A spontaneous excitation of the entire domain leads to the the spiral wave termination. D) Voltage-time series of a point in the domain before, during and after illumination.

a line in the range (shown in Fig.3.1-A) from sub-threshold LIs, $\leqslant 20 \mu \mathrm{W} / \mathrm{mm}^{2}$ to supra-threshold LIs, $\geqslant 25 \mu \mathrm{W} / \mathrm{mm}^{2}$. We see that for the sub-threshold illumination, increasing the LI increases the period of the spiral wave $\left(\mathrm{T}_{s}\right)$, which leads to a decrease in its conduction velocity $(\mathrm{CV})$. In the supra-threshold régime, at low LI (between $25-50 \mu \mathrm{W} / \mathrm{mm}^{2}$ ) an excitation wave is created at the core region which propagates through the whole domain and excites it to an elevated constant potential. Increasing LI leads to faster initiation of the excitation wave, reaching the elevated constant potential at shorter transient times. At very high $\mathrm{LI},\left(\geqslant 80 \mu \mathrm{W} / \mathrm{mm}^{2}\right)$ the system immediately enters the elevated constant potential when the light is switched on.

These different termination mechanisms in low and high LIs may be explained by the kinetics of ChR2 and its characterization activation at different LIs. The activation of this protein leads to three different phases: 1) an initial sharp peak of the incoming cation current $\left(\mathrm{I}_{\text {peak }}\right), 2$ ) decay of this peak to a constant phase with the width of the optical pulse duration $\left(\mathrm{I}_{\text {cnst }}\right)$, and 3) decay of the current to the baseline when the light is switched off. The application of low LIs results in slow activation of this channel with low $\mathrm{I}_{\text {peak }}$ and $\mathrm{I}_{\text {cnst }}$, so that the arrhythmia can be terminated with a longer optical PL. This leads to a transient termination time, which causes a few turns of the spiral wave. On the other hand, with a high LI this channel is activated immediately and within a short time a large amount of $\mathrm{I}_{\text {peak }}$ flows. This leads to immediate excitation of the heart tissue, which can lead to an abrupt termination without a transient time. 


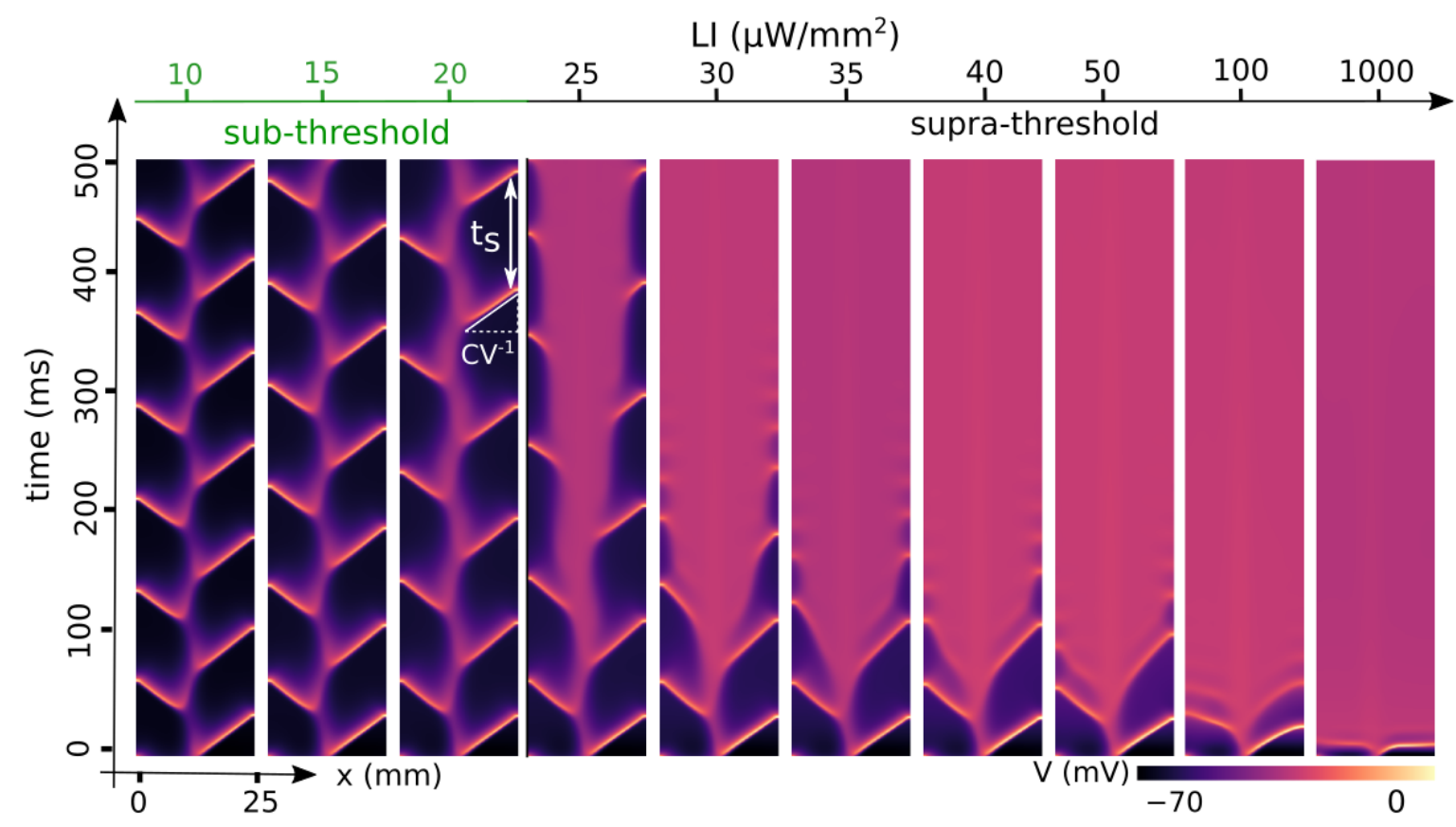

Figure 3.3 - Core dissolution of a spiral wave. Space-time plots of the membrane voltage across a line, shown as a dashed line in Fig.3.1-A, in a $2 D$ domain with an existing spiral wave during $500 \mathrm{~ms}$ of a single pulse global illumination from sub to supra-threshold LIs. At sub-threshold illumination $\left(\leqslant 20 \mu \mathrm{W} / \mathrm{mm}^{2}\right)$ the period of the spiral wave $\left(T_{s}\right)$ and the conduction velocity $(\mathrm{CV})$ increases by increase of the LI. At supra-threshold LI ( $\geqslant 25$ $\mu \mathrm{W} / \mathrm{mm}^{2}$ ) the core region which is more susceptible compare to the rest of the domain, is excited first and an excitation wave is propagated towards the entire domain. This results in a progressive termination with a transient time. In which the increase of LI leads to a decrease of the transient termination time.

To investigate the transition dynamics into and out of the core dissolution process, we applied a shorter PL, $150 \mathrm{~ms}$, to the same range of LIs. Fig.3.4 shows that at LIs below the threshold, the CV of the wave is reset to its initial velocity. The dissolution process of the core at LIs of $25 \mu \mathrm{W} / \mathrm{mm}^{2}$ and $30 \mu \mathrm{W} / \mathrm{mm}^{2}$ is stopped when the light is turned off. At LI of 25, the spiral wave continues to rotate at the same point, but at LI of $30 \mu \mathrm{W} / \mathrm{mm}^{2}$, the core of the spiral wave is shifted to another part of the range. At $\mathrm{LI} \geqslant 35 \mu \mathrm{W} / \mathrm{mm}^{2}$, the core dissolution continues and leads to the termination of the spiral wave.

In the conventional defibrillation method, the arrhythmia termination rate is dependent on the electric field strength, which can be reflected in a plot of the doseresponse curve [6]. The sigmoidal behaviour of the dose-response curve reflects the lower probability of termination at reduced field strength, while at large electric field amplitude the termination rate is increased close to $100 \%$. In this study, we investigate the behaviour of the dose-response curve when a single global optical pulse is applied to the spiral wave at different LIs and PLs of 10, 100 and $300 \mathrm{~ms}$.

Fig.3.5-A shows a similar curve of the dose-response diagram with the optical 


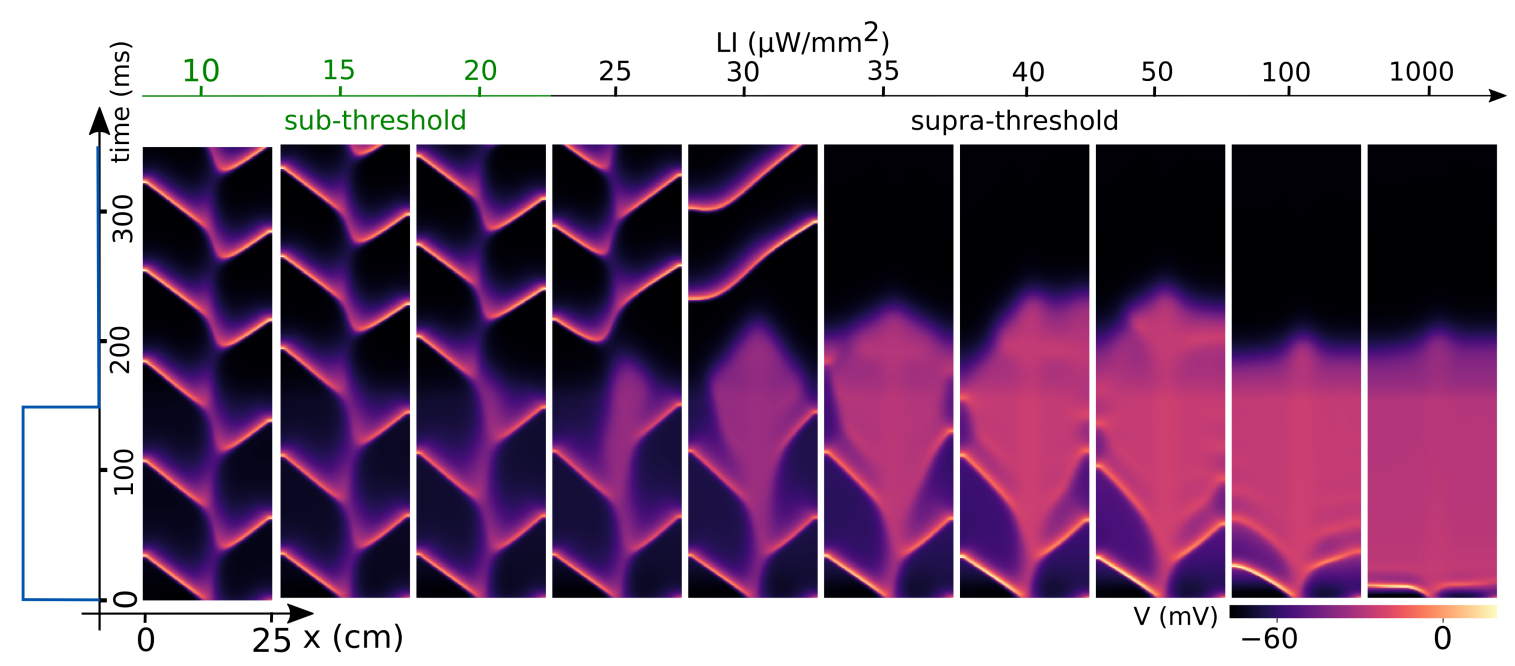

Figure 3.4 - Space-time plots of the membrane voltage across a line, shown as a dashed line in Fig.3.1-A, in a 2D domain with an existing spiral wave. A global pulse illumination with pulse length (PL) of $150 \mathrm{~ms}$ (shown with a blue trace on the left) was applied at different light intensities (LIs) from sub $\left(<25 \mu \mathrm{W} / \mathrm{mm}^{2}\right)$ to supra-threshold ( $>25$ $\left.\mu W / \mathrm{mm}^{2}\right)$ LIs.

stimulation compared to the electrical stimulation method. Increasing LI and LI leads to an increase in arrhythmia termination efficiency. The termination time for the successful termination cases demonstrated in Fig.3.5-B shows that the termination transient time is more than $100 \mathrm{~ms}$ at low LIs $\left(50 \mu \mathrm{W} / \mathrm{mm}^{2}\right)$ due to the dissolution core process. However, at high LIs $\left(\geqslant 80 \mu \mathrm{W} / \mathrm{mm}^{2}\right)$, due to the abrupt annihilation, the termination time is much shorter and varies in a narrow range of 10 to $20 \mathrm{~ms}$.

A similar trend of the dose-response curve is observed in the experiments on the intact mouse heart (Fig.3.5-C) at different LIs and PLs of 10, 100 and $500 \mathrm{~ms}$. The transient termination time of the experimental data shown in Fig.3.5-D is in quantitative agreement with the numerical study. Although the two systems, one a 2D simulation of heart tissue and the other an intact mouse heart, are very different, but the similar trend in termination transient time in each case suggests that the similarity may be due to a common feature of the two systems. Sharing the kinetics of ChR2 could be a possible explanation of such similarity in numerical and experimental studies. Due to the fast activation of this channel, within $\approx 25 \mathrm{~ms}$, and large amount of $\mathrm{I}_{\text {peak }}$ at high LIs termination occurs immediately at very short transient termination time. However at low LIs the ChR2 is activated slower with a lower amount of the $\mathrm{I}_{\text {peak }}$. This leads to longer transient termination time during which $\mathrm{I}_{\text {peak }}$ and $\mathrm{I}_{\text {cnst }}$ act together to terminate the spiral wave activity in the domain.

Finally, we compare the electrical activity of the intact cardiac tissue when a single global illumination pulse is applied during an arrhythmic state of the heart at two different lowest and highest LIs. Fig.3.5-E and F show a signal from the monophasic action potential (MAP) recording during $500 \mathrm{~ms}$ illumination with the LIs of 20 $\mu \mathrm{W} / \mathrm{mm}^{2}$ and high LI of $560 \mu \mathrm{W} / \mathrm{mm}^{2}$, respectively. The red shaded box shows the 
termination time, which is longer for the case of low LI compared to high LI. Thus, our experimental data supports our numerical observation shown in Fig.3.2-B and D.

\section{Conclusion}

Ongoing studies on the development of alternative treatment methods that have fewer negative side effects compared to the conventional techniques, [6] show that the use of lower field strength leads to a successful termination with a certain probability that is proportional to the electric field strength. This points to the possibility that the electric field does not excite the entire tissue but only a part of it, which leads to a lower number of field-activated heterogeneities. These heterogeneities act as secondary wave sources under electric field stimulation [69]. In this study, we used optogenetics with a protocol similar to the conventional defibrillation method. We report a transition of the termination mechanism by increasing the light intensity. At low LIs we observe a progressive termination of the spiral wave starting from the core region. Due to the lack of excitation activity the core region is more susceptible to be excited. This results in core dissolution, followed by initiation and propagation of an excitation wave from that region. This wave pushes the arm toward the inexcitable boundary by spreading through the entire domain. The process of core dissolution at low LIs takes a longer termination time compared to the abrupt termination at high LIs. The experimental data of this study supports our numerical observations.

The signal of the electrocardiogram (ECG) gives us information about the heart status during sinus rhythm or in case of cardiac arrhythmia such as VF. Due to the loss of information of the ECG signal during electrical defibrillation, the ECG data lack some information that could help with understanding the mechanism underlying defibrillation. Recently, optogenetics has made it possible to record the electrical activity of the heart during the optical stimulation. Numerous studies have been carried out to control cardiac arrhythmias in murine hearts with different lighting patterns, such as structured [21, 22, 99] and global [23]. R. Q. Uribe et al. [23] applied global illumination to control cardiac arrhythmias in intact transgenic mouse hearts at different PL and LI. They showed for some successful termination cases that there is a non-spontaneous termination at low LI with a long PL of 1000 ms. Our numerical studies suggest that tip disintegration may be the underlying mechanism leading to the non-spontaneous termination that they have observed for the long illumination pulse with low-intensity. 

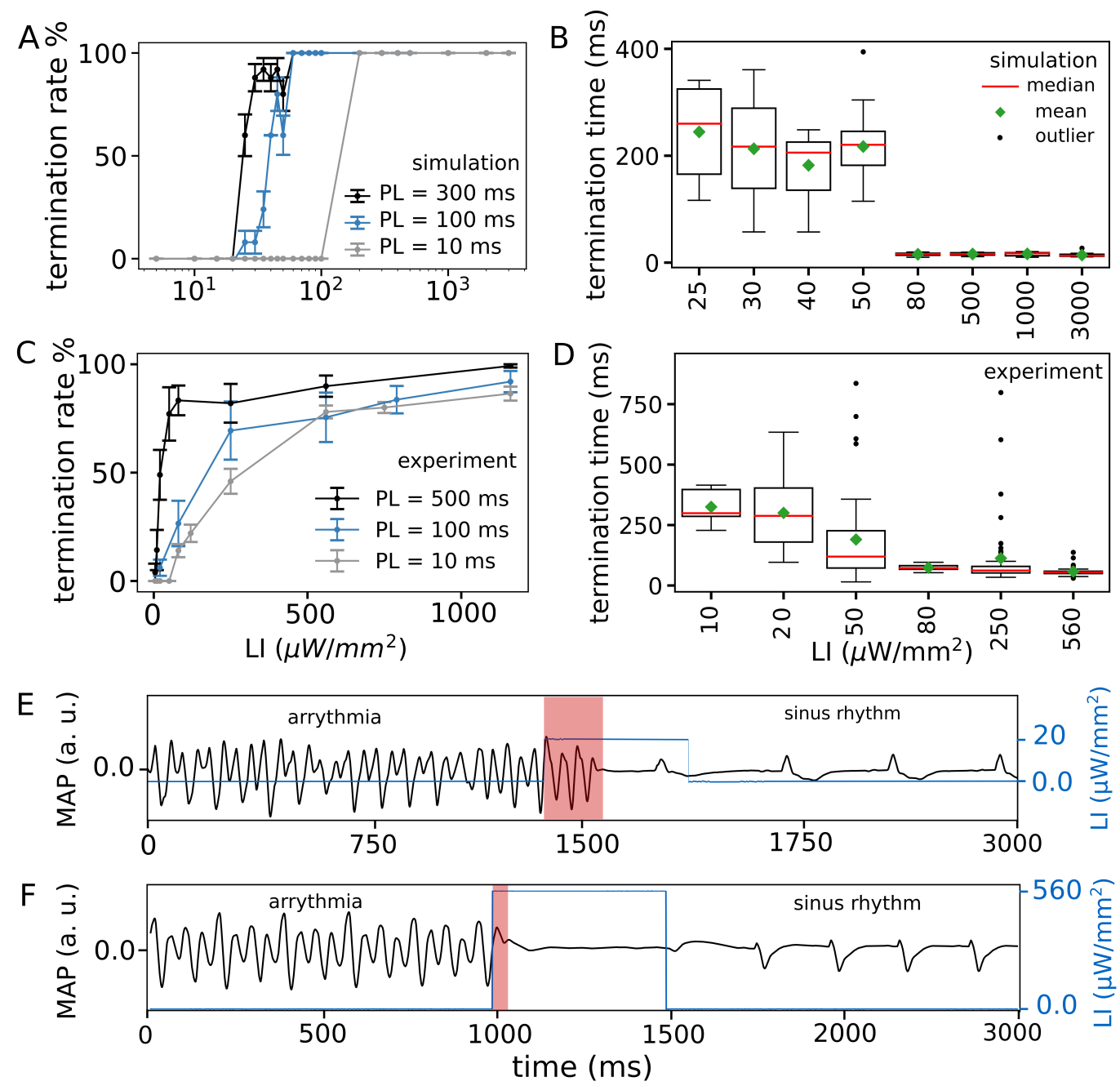

Figure 3.5 - Termination of arrhythmia applying a single pulse global illumination in silico and ex vivo. A) Termination rate of a single spiral wave in a $2 D$ domain with size of $25 \mathrm{~mm} \times 25 \mathrm{~mm}$ simulation $(\mathrm{N}=25)$ at different $\mathrm{LI}$ and $\mathrm{PL}$ (percentage of successful attempts reported as mean \pm SEM). B) Termination time at different LIs with PL of 300 ms. At the very low supra-threshold LIs $\left(<50 \mu \mathrm{W} / \mathrm{mm}^{2}\right)$ termination time is more than $100 \mathrm{~ms}$. However at the high supra-threshold LIs, termination time is shorter and varies in a narrow range of $(10,20) \mathrm{ms} . C)$ Arrhythmia termination rate vs. light intensity (LI) in Langendorff-perfused intact mouse hearts $(N=5)$ for pulse lengths $(P L)$ of 10, 100, and $500 \mathrm{~ms}$ (percentage of termination rate reported as mean $\pm S E M)$. Increase of LI and $P L$ leads to increase of termination rate. D) Termination time at different $L I s$ and $P L$ of $500 \mathrm{~ms}$. It shows decrease of termination time for increasing LI, so that at low LI termination $\left(<80 \mu \mathrm{W} / \mathrm{mm}^{2}\right)$ time time is longer that high $\mathrm{LI}\left(>250 \mu \mathrm{W} / \mathrm{mm}^{2}\right)$. E) and $F$ ) Monophasic action potential (MAP) recording of the intact mouse heart before, during, and after illumination. It shows arrhythmia termination during a single pulse global illumination. Termination time for the case of $L I=20 \mu \mathrm{W} / \mathrm{mm}^{2}$, shown with a red-shaded box in (E) is longer than the case of $L I=560 \mu \mathrm{W} / \mathrm{mm}^{2}$ shown in $(F)$. 



\section{Chapter 4}

\section{Drift and termination of spiral waves in optogenetically modified cardiac tissue at sub-threshold illumination}

\subsection{Scientific goal and findings}

The formation of spiral waves in the heart is the main cause of life-threatening cardiac arrhythmias. Due to the side effects of strong, globally restoring electric shocks, there are attempts to develop clinical low-energy defibrillation techniques. Further development of clinical applications are based on our understanding of the nonlinear dynamics underlying the scroll and spiral waves in the heart. Cardiac optogenetics opens up a promising avenue to study the dynamics and control of spiral waves in the heart.

In this work, I mainly use simulations to study the dynamic control of spiral waves rotating in a 2D domain representing the ventricular tissue of an adult mouse heart. The results of this study postulate another underlying mechanism of the optogenetic defibrillation method. To control the wave dynamics, I applied different illumination patterns below the threshold, a linear gradient over the entire domain, a one-step illumination in half the domain, and a multi-step adjusted illumination. In all cases the spiral wave drifts towards the region with higher LI. This observation can be extended to a realistic geometry of the heart if a global epicardial illumination is applied. Exponential decay of the illumination across the wall can cause the drift of a spiral wave rotating below the heart surface towards the epicardium.

In addition to these simulations, I performed and analyzed some experimental studies with a master student during her lab rotation to investigate the effect of low LI on arrhythmia frequency in an intact mouse heart. The experimental study of the $\mathrm{CV}$ in an intact mouse heart was performed by our colleagues in Italy and Canada. All experimental data provided show the main effect of light intensity on heart rhythm 
Drift and termination of spiral waves in optogenetically modified cardiac tissue at sub-threshold illumination

and arrhythmia frequency and confirm the numerical observations.

\section{Manuscript Status}

Accepted at eLife.

\section{Author Contributions}

- Sayedeh Hussaini designed the research, performed all numerical simulations, analyzed the experimental and numerical data, and wrote the manuscript

- Vishalini Venkatesan performed the experiments of the intact mouse heart to study the arrhythmia frequency, analyzed the data, and wrote the manuscript

- Valentina Biasci performed the experiments of the intact mouse heart to study the $\mathrm{CV}$, analyzed the data, and wrote the manuscript

- Jose M. Romero Sepulveda performed the experiments in the cell culture to study the arrhythmia frequency, analyzed the data, and wrote the manuscript

- Raul A. Quinonex Uribe designed the experimental setup for global illumination of the intact mouse heart

- Leonardo Sacconi performed experiments of the intact mouse heart to study CV were conducted in his lab and edited the manuscript

- Gil Bub performed experiments in the cell culture were conducted at his lab and edited the manuscript

- Claudia Richter performed experiments of the intact mouse heart to study the arrhythmia frequency were conducted at her lab and edited the manuscript

- Valentin Krinski designed the research and edited the manuscript

- Ulrich Parlitz designed the research and edited the manuscript

- Rupamanjari Majumber designed the research and wrote the manuscript

- Stefan Luther designed the research and wrote the manuscript

\subsubsection{Manuscript}

Hussaini, S., Venkatesan, V., Biasci, V., Romero Sepúlveda, J.M., Uribe, R.A.Q., Sacconi, L., Bub, G., Richter, C., Krinski, V., Parlitz, U., Majumder, R. and Luther, S., 2020. Drift and termination of spiral waves in optogenetically-modified cardiac tissue at sub-threshold illumination. bioRxiv doi: https://doi.org/10.1101/2020.06.12.148734. 


\title{
Drift and termination of spiral waves in optogenetically modified cardiac tissue at sub-threshold illumination
}

\author{
Sayedeh Hussaini ${ }^{1,2,3}$, Vishalini Venkatesan ${ }^{1,4}$, Valentina Biasci ${ }^{5,6}$, \\ José M Romero Sepúlveda ${ }^{7}$, Raul A Quiñonez Uribe ${ }^{1,3}$, Leonardo Sacconi ${ }^{5,8,9}$, \\ Gil Bub7 , Claudia Richter ${ }^{1,3,4}$, Valentin Krinski ${ }^{1,3,10}$, Ulrich Parlitz ${ }^{1,2,3}$, \\ Rupamanjari Majumder ${ }^{1,3}$, Stefan Luther ${ }^{1,2,3,11 *}$
}

${ }^{1}$ Research Group Biomedical Physics, Max Planck Institute for Dynamics and SelfOrganization, Goettingen, Germany; ${ }^{2}$ Institute for the Dynamics of Complex Systems, Goettingen University, Goettingen, Germany; ${ }^{3}$ German Center for Cardiovascular Research, Partner Site Goettingen, Goettingen, Germany; ${ }^{4}$ University Medical Center Goettingen, Clinic of Cardiology and Pneumology, Goettingen, Germany; ${ }^{5}$ European Laboratory for Non-Linear Spectroscopy, Sesto Fiorentino (FI), Italy; ${ }^{6}$ Division of Physiology, Department of Experimental and Clinical Medicine, University of Florence, Florence, Italy; ${ }^{7}$ Department of Physiology, MGill University, Montreal, Canada; ${ }^{8}$ Institute for Experimental Cardiovascular Medicine, University of Freiburg, Freiburg, Germany; ${ }^{9}$ National Institute of Optics, National Research Council, Florence, Italy; ${ }^{10}$ INPHYNI, CNRS, Sophia Antipolis, Paris, France; ${ }^{11}$ University Medical Center Goettingen, Institute of Pharmacology and Toxicology, Goettingen, Germany

*For correspondence: stefan.luther@ds.mpg.de Competing interests: The authors declare that no competing interests exist.

Funding: See page 14

Received: 12 June 2020 Accepted: 28 November 2020

Reviewing editor: Franziska Schneider-Warme, University of Freiburg, Germany

(c) Copyright Hussaini et al. This article is distributed under the terms of the Creative Commons Attribution License, which permits unrestricted use and redistribution provided that the original author and source are credited.
Abstract The development of new approaches to control cardiac arrhythmias requires a deep understanding of spiral wave dynamics. Optogenetics offers new possibilities for this. Preliminary experiments show that sub-threshold illumination affects electrical wave propagation in the mouse heart. However, a systematic exploration of these effects is technically challenging. Here, we use state-of-the-art computer models to study the dynamic control of spiral waves in a two-dimensional model of the adult mouse ventricle, using stationary and non-stationary patterns of sub-threshold illumination. Our results indicate a light-intensity-dependent increase in cellular resting membrane potentials, which together with diffusive cell-cell coupling leads to the development of spatial voltage gradients over differently illuminated areas. A spiral wave drifts along the positive gradient. These gradients can be strategically applied to ensure drift-induced termination of a spiral wave, both in optogenetics and in conventional methods of electrical defibrillation.

\section{Introduction}

Emergence of reentrant electrical activity, often in the form of spiral and scroll waves, is associated with the development of life-threatening heart rhythm disorders, known as cardiac arrhythmias (Krinski, 1968; Davidenko et al., 1990; Davidenko et al., 1992; Pertsov et al., 1993). These abnormal waves stimulate the heart to rapid, repetitive and inefficient contraction, either in a periodic manner, as in the case of monomorphic ventricular tachycardia (mVT) (Cysyk and Tung, 2008), or in a quasiperiodic to chaotic manner, as in the case of polymorphic ventricular tachycardia (pVT) and fibrillation (Antzelevitch and Burashnikov, 2001). The state-of-the-art technique for controlling the dynamics of these abnormal waves involves global electrical synchronization. This is achieved by 
applying high-voltage electric shocks to the heart (Wathen et al., 2004). However, such shocks are often associated with severe side effects, such as unwanted tissue damage (Babbs et al., 1980) and the development of mental disorders such as anxiety and depression in patients who experience intense pain and trauma each time the shock is delivered (Newall et al., 2007; de Ornelas Maia et al., 2013). Therefore, alternative low-energy approaches for treatment are in great demand.

One low-energy technique to control arrhythmias in the clinical setting is anti-tachycardia pacing (ATP) (Wathen et al., 2004). A biomedical device such as a standard implantable cardioverter defibrillator (ICD) is designed to detect the occurrence of an arrhythmia. The ATP method is based on coupling this property of the device to a local source that sends a train of electric waves in the heart to drive the spiral wave in a desired direction (Bittihn et al., 2008). In a finite domain, the forced drift eventually causes the phase singularity at the tip of the spiral wave to collide with an inexcitable boundary, ensuring its elimination (Gottwald et alo, 2001). Despite its ability to control mVT and pVT (Wathen et alo, 2004), the ATP method proves to be sub-optimal in controlling high-frequency arrhythmias and arrhythmias associated with pinned spiral waves (Pumir et al., 2010). Subsequent improvements by Fenton et al., 2009, Luther et al., 2011, Li et al., 2009 and Ambrosi et al., 2011 reduced the defibrillation threshold and fatal side effects. Further progress in the clinical implementation of these developing techniques requires a deeper understanding of the underlying spiral and scroll wave dynamics.

Recently, optogenetics has emerged as a promising tool for studying wave dynamics in cardiac tissue, overcoming some major challenges in imaging and probing (Deisseroth, 2011). In particular, its capabilities have been extensively used to study the mechanisms underlying the incidence, maintenance, and control of cardiac arrhythmias (Bruegmann et al., 2010; Nyns et al., 2017; Crocini et al., 2017; Quiñonez Uribe et al., 2018), and to address questions of a fundamental nature, for example the possibility to exercise control over the chirality (Burton et al., 2015) and core trajectories (Majumder et al., 2018) of spiral waves. All these studies demonstrate manipulation or abrupt termination of spiral waves by supra-threshold optical stimulation, that is stimulation that has the ability to trigger action potentials in individual cells and initiate new waves in extended media. However, very little is known about the use of optogenetics in the sub-threshold stimulation régime, which is why we have decided to investigate it in our present work. To demonstrate the drift of spiral waves using sub-threshold illumination, we use a two-dimensional (2D) continuum model of the cardiac syncytium, containing ionically-realistic representations of optogenetrically modified adult mouse ventricular cardiomyocytes at each node of the simulation domain. Sub-threshold illumination causes a shift in the resting membrane potential of optically modified heart cells without triggering action potentials. This shift affects the conduction velocity (CV) and wavelength of the propagating waves and allows spatiotemporal control of spiral wave dynamics. By applying patterned sub-threshold illumination with light intensity (LI) that is a function of space, we impose a spatial gradient on the recovery state of individual cells that make up the domain. This leads to a drift of the spiral wave along the direction of slower recovery. We show how this method can be used to ensure drift and termination of spiral waves in cardiac tissue.

\section{Results}

In cardiac tissue, the level of electrochemical stimulation required to induce an action potential, is called the excitation threshold. Application of external stimulation below this threshold, causes small positive increase in the membrane voltage, which is insufficient to produce new waves. In this study, we use optogenetics at sub-threshold Lls to investigate the possibility of controlling spiral wave dynamics in light-sensitive cardiac tissue.

We begin with a study of the effect of uniform, global, constant sub-threshold illumination at different $\mathrm{LI}$ on the conduction velocity (CV) of plane propagating waves in a $2.5 \mathrm{~cm} \times 0.25 \mathrm{~cm}$ pseudo domain. We find that, for electrically paced waves, CV shows a dependence on the pacing cycle length $(C L)$ only when the $C L$ is low $(<200 \mathrm{~ms})$. The $C V$ restitution curve begins to flatten around a $\mathrm{CL}=200 \mathrm{~ms}$, for all LI (Figure 1A). In particular, for electrical pacing at $5 \mathrm{~Hz}, \mathrm{CV}$ shows an approximate $5 \%$ decrease as $\mathrm{LI}$ is increased from 0 (no illumination) to $0.02 \mathrm{~mW} / \mathrm{mm}^{2}$ (Figure 1B). This decrease in CV may be attributed to the limited availability of $\mathrm{Na}^{+}$channels at elevated membrane voltages. In experiments, a decrease in CV was observed, with increase in LI. The reduction was two 

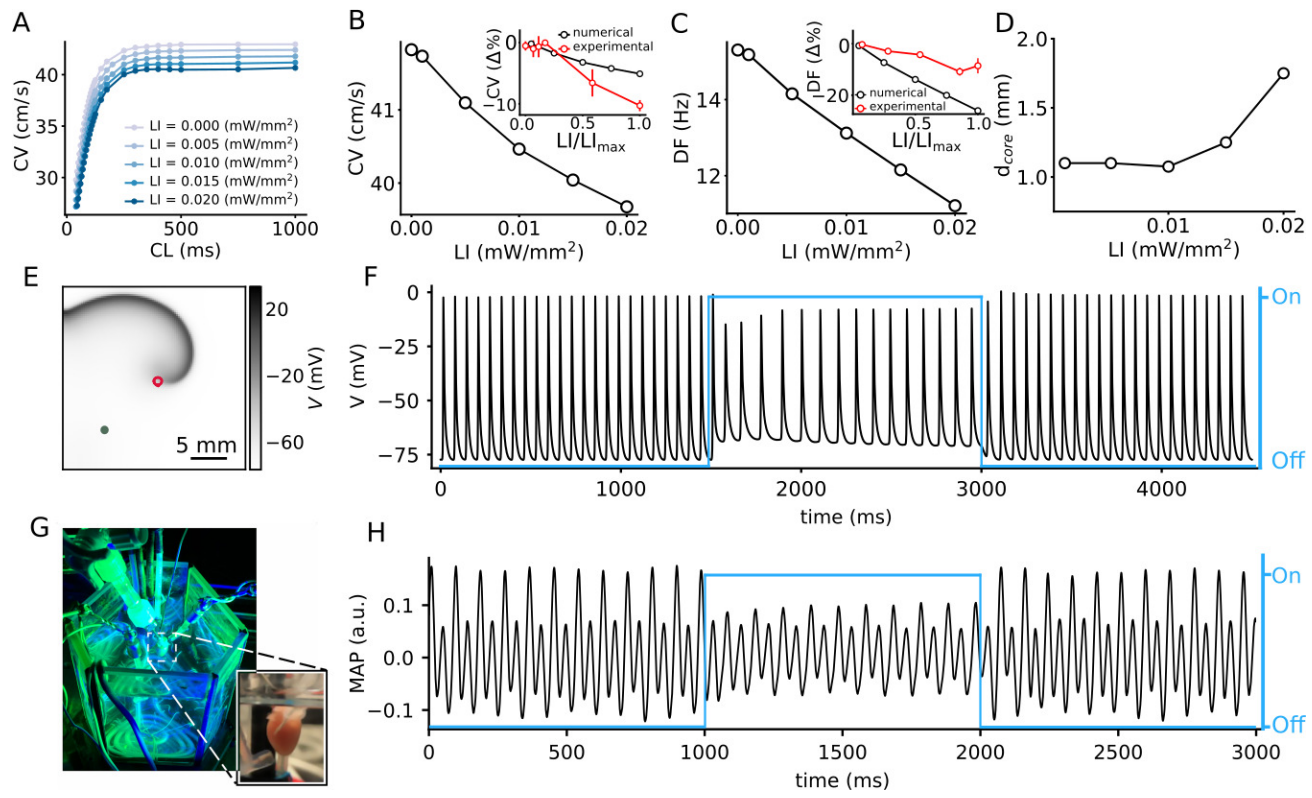

$\mathrm{H}$

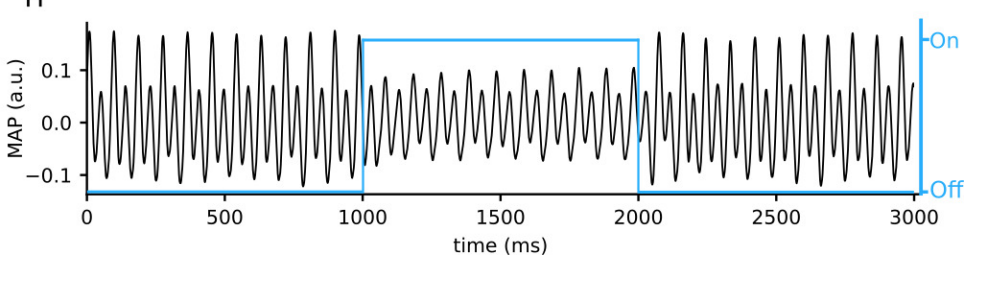

Figure 1. Effect of sub-threshold illumination on in silico optogenetically modified adult mouse ventricular tissue. (A) Conduction velocity (CV) restitution at different light intensities (LIs). (B) CV decreases with increase in LI, for electrical excitation waves paced at $5 \mathrm{~Hz}$. Inset shows a comparison of the reduction of $\mathrm{CV}$ in experiments (red) and simulations (black) at different LI, relative to the unilluminated planar wave (CV reported as mean $\pm \mathrm{SEM}, \mathrm{N}=4$ with 12 trials). (C) Dominant frequency (DF) of a spiral wave decreases with increase in LI. Inset shows a comparison of the reduction of DF in experiments (red) and simulations (black) at different LI, relative to the unilluminated spiral (DF reported as mean $\pm S D, N=2$ with 14 trials). (D) Increase in diameter of the spiral wave core $\left(d_{c o r e}\right)$ with increase in LI. Here, core represents the circle that encloses one cycle of the spiral tip trajectory in the stationary state. (E) A representative snapshot of the spiral wave in a $2 \mathrm{D}$ simulation with a circular trajectory shown with red marker. The green marker indicates the location for extraction of the voltage timeseries in (F). (G) Our set-up of the intact mouse heart from which monophasic action potential (MAP) recordings in $(\mathrm{H})$ were made. The blue traces in $(\mathrm{F})$ and $(\mathrm{H})$ illustrate the status of illumination (on/off) during the simulation or experiment.

The online version of this article includes the following figure supplement(s) for figure 1:

Figure supplement 1. (A) Four different membrane voltage values are chosen; one value from resting state (1), one value from depolarization state (2), and two values from repolarization state $(3$ and 4). (B) the $2 D$ mono-domain $(200 \times 200)$ is initialized by four selected values. (C) A spiral wave is formed. (D) shows a series of frames during formation of the spiral wave.

times more than in simulations, at the highest sub-threshold LI for intact mouse hearts $(\simeq 0.15 \mathrm{~mW} /$ $\mathrm{mm}^{2}$ ) shown as an inset of Figure $1 B$.

In 2D cardiac tissue, sub-threshold illumination seems to have a profound influence on the frequency of a spiral wave. Many theoretical and numerical studies have shown that heterogeneity in an excitable medium can cause a spiral wave to drift. This drift has a temporal component that is associated with a change in the rotation frequency of the spiral wave, and, a spatial component that is associated with the motion of the rotation center, or core, of the spiral wave (Krinski, 1968; Biktasheva et al., 2010). In the absence of light, our spiral wave rotates periodically with a temporal frequency of $\simeq 15.3 \mathrm{~Hz}$, and a circular core trajectory. We apply uniform, global, constant subthreshold illumination at $\mathrm{LI} \leq 0.02 \mathrm{~mW} / \mathrm{mm}^{2}$, for $1500 \mathrm{~ms}$. Power spectra calculated from the voltage timeseries $V(0.75 \mathrm{~cm}, 1.75 \mathrm{~cm}, t)$ shows periodic readouts with a single dominant frequency (DF) for each LI. We find that DF decreases with increase in LI (Figure 1C). In particular, we observe a $26 \%$ reduction in the DF in simulations, in going from no illumination, to $\mathrm{LI}=0.02 \mathrm{~mW} / \mathrm{mm}^{2}$. In experiments on the intact mouse heart, a decrease in DF reduction was observed, with increase in Ll; however, the reduction was two times less than in simulations, at the highest LI for intact mouse hearts $\left(0.015 \mathrm{~mW} / \mathrm{mm}^{2}\right)$ shown as an inset of (Figure 1C). Application of sub-threshold light stimulation did not alter the general shape of the spiral tip trajectory. It remained circular at all LI considered. However, the core diameter gradually increased with increase in LI (Figure 1D). A representative 
snapshot of the spiral wave in a simulation domain with uniform, global sub-threshold illumination at $\mathrm{LI}=0.02 \mathrm{~mW} / \mathrm{mm}^{2}$ is shown in Figure $1 E$, with the corresponding voltage timeseries $V(0.75 \mathrm{~cm}$, $0.75 \mathrm{~cm}, \mathrm{t}$ ) in Figure $1 \mathrm{~F}$.

Similar temporal drift is observed in experiments on the intact mouse heart (Figure 1G and $H$ ) at $\mathrm{LI}=0.015 \mathrm{~mW} / \mathrm{mm}^{2}$. Thus, the experimental data supports our finding that the period of the spiral can be increased in the presence of the illumination. It is important to note that the effect of the illumination is reversible, as is demonstrated by the voltage timeseries in $(G)$ and $(H)$, which show that the natural rotation frequency of the spiral can be restored upon removal of the light stimulus. To summarize, our results provide substantial evidence to support the change in spiral wave frequency, the so-called temporal drift, in response to uniform global constant sub-threshold illumination.

Intrinsic inhomogeneity of cardiac tissue can cause a spiral wave to drift. Such inhomogeneity can be induced using sub-threshold illumination. In order to investigate the possibility of the induction of spatial drift of a spiral wave using sub-threshold illumination, we generate a spiral wave in the nonilluminated 2D domain, and use it to define the configuration of the system at $t=0 \mathrm{~s}$. We apply a linear gradient of sub-threshold illumination to this spiral wave. Figure $2 \mathrm{~A}$ shows the spiral at $t=2 \mathrm{~s}$, when the applied linear gradient in $\mathrm{LI}$ ranges from $0 \mathrm{~mW} / \mathrm{mm}^{2}$, to $0.01 \mathrm{~mW} / \mathrm{mm}^{2}$, across the length of the domain in the x-direction. In all the observed cases, the stationary spiral wave drifts toward the region with high resting membrane potential, which corresponds to the region with high LI. The inhomogeneity in the distribution of the resting membrane potential of cardiac cells across the domain establishes over time when the domain is exposed to light. We demonstrate the spatiotemporal evolution of this 'quiescent' membrane voltage $(V)$, along the line $y=0.75 \mathrm{~cm}$ on the $2 \mathrm{D}$ domain, perpendicular to the illumination pattern (shown as a dot-dashed line in Figure 2A), in response to constant subthreshold illumination, in the absence of any electrical activity in the domain (see Figure 2B). The spatiotemporal evolution of the magnitude of the spatial derivative of the $V$ $(d V / d x)$ in shown in Figure 2C. Each trace represents the voltage profile along the dot-dashed line in the quiescent domain, at times corresponding to that indicated on the color bar given alongside. We have ensured that each trace in Figure $2 \mathrm{~B}$ and Figure $2 \mathrm{C}$ represents the quiescent voltage profile of the domain at times which would correlate with successive turns of a spiral wave, drifting within the domain, in response to the establishing light-induced inhomogeneity. Figure 2D-F show the corresponding results obtained with a linear $\mathrm{LI}$ gradient ranging from $0 \mathrm{~mW} / \mathrm{mm}^{2}$, to $0.02 \mathrm{~mW} /$ $\mathrm{mm}^{2}$, in the x-direction. In this case, we observe drift-induced termination of the spiral wave (see Video 1). It is important to note that establishment of the voltage gradients in Figure $2 B$ and $E$ impose a spatial non-uniformity in the refractory period of cells that constitute the domain. Regions with higher $\mathrm{LI}$ experience higher shifts in membrane voltage compared to regions with lower $\mathrm{LI}$, and consequently display longer refractory period. Thus, irrespective of the range of $\mathrm{LI}$ used to produce the applied light gradient, the spiral wave always drifts along the direction of the longer refractory period, that is, higher LI (Panfilov, 2009). Figure 2G shows the instantaneous speed of the spiral tip (red), as a function of time, during 1000 to $1250 \mathrm{~ms}$, corresponding to the spiral wave trajectory shown as inset in Figure 2D. The gray zones in Figure 2G indicate drift against the gradient, whereas, the green zones indicate the drift along the direction of the applied gradient in LI. The same figure also shows the instantaneous curvature of the tip trajectory (black). Each peak of the curvature curve corresponds to a minimum value of the speed showing drift of the spiral, against the gradient. Finally, Figure $2 \mathrm{H}$ shows the horizontal displacement of the spiral core relative to its initial position (i.e. the center of the domain), at the end of $2 \mathrm{~s}$ of simulation. We observe that within the given time frame spiral termination occurs only for the highest $\mathrm{LI}$ gradient used. The main advantage of this method is that termination can occur irrespective of the initial position of the spiral wave core.

Inspired by the success of this method to ensure drift-induced termination of spiral waves, we now work to optimize the protocol to increase the drift velocity with which the spiral wave can approach the boundary. We replace the smooth gradient illumination pattern by a step-like distribution of LI. We apply uniform sub-threshold illumination to one half of the domain such that the spiral wave core is located at the interface between illuminated, and non-illuminated regions, where the spatial derivative of the membrane voltage, in the quiescent state, is highest (see Video 2). Figure 3A shows the migration of the spiral wave from interface toward the illuminated region during $2 \mathrm{~s}$ of the illumination. Typically, the drift velocity of the spiral tip in the initial phase is proportional to the slope of the gradient at the interface. However, as the spiral migrates along the 

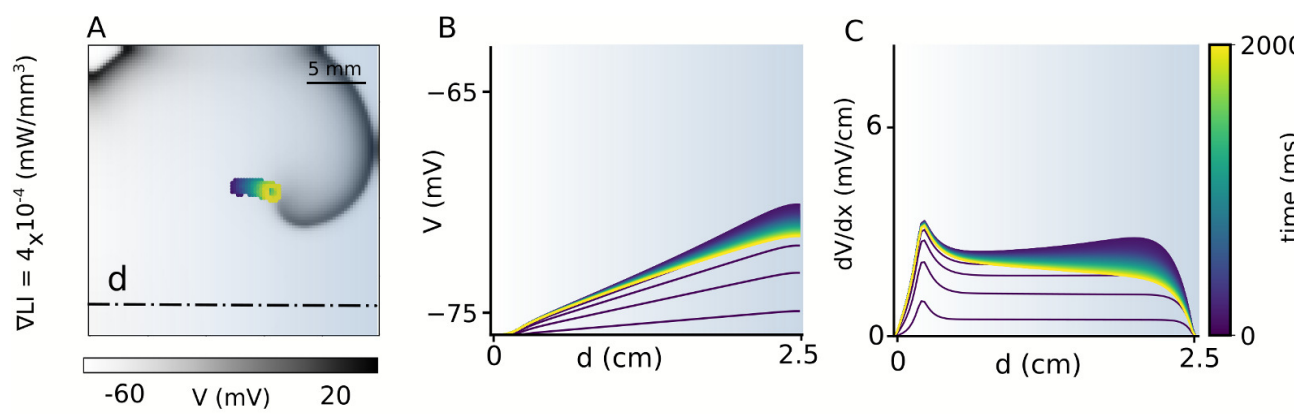

D

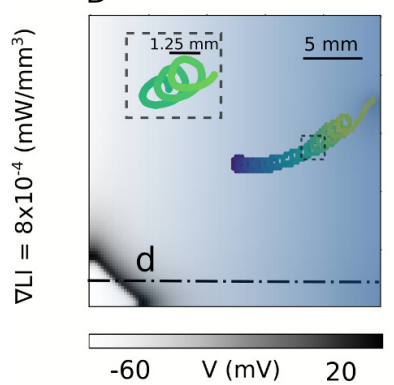

$\mathrm{E}$

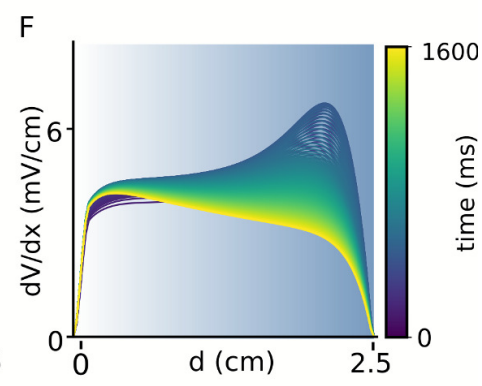

G
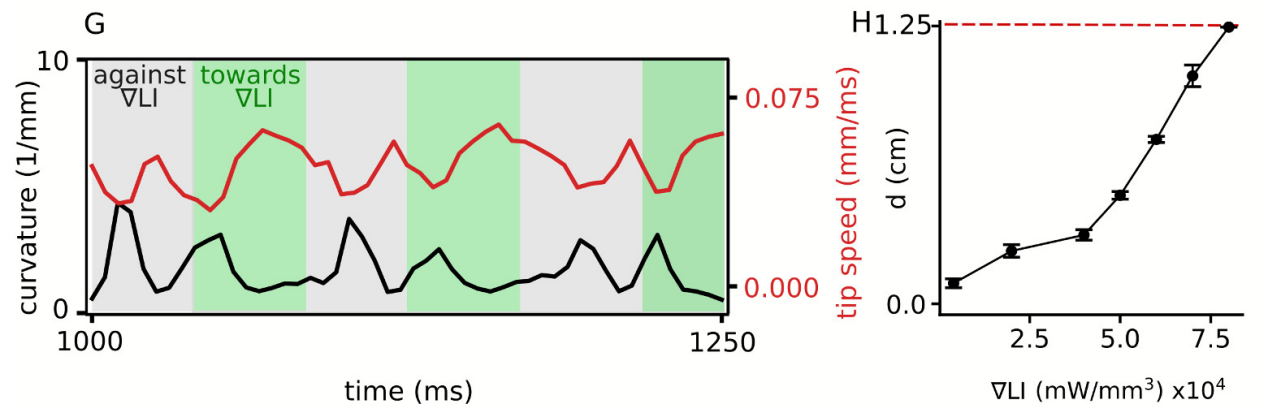

Figure 2. Spatial drift of a spiral wave imposed by a gradient of sub-threshold illumination. (A) Trajectory of a drifting spiral tip in a domain with an illumination gradient ranging from $\mathrm{LI}=0 \mathrm{~mW} / \mathrm{mm}^{2}$ at the left boundary to $\mathrm{LI}=0.01 \mathrm{~mW} / \mathrm{mm}^{2}$ at the right boundary. Colors indicate different times, here and B-F. (B) Time evolution of the voltage distribution along the dashed line indicated in (A), in a quiescent domain with the same LI gradient. (C) Spatial derivative of the voltage distribution $(d V / d x)$ along the dashed line in $(\mathrm{A})$, at different times, for the same applied $\mathrm{LI}$ gradient as in $(\mathrm{A}-\mathrm{B})$. (D)-(F) show plots corresponding to (A)-(C), but for an LI gradient ranging from 0 to $0.02 \mathrm{~mW} / \mathrm{mm}^{2}$. In this case, the spiral drifts all the way to the right boundary, within the given time frame, and terminates itself. The inset in (D) shows a portion of the cycloidal tip trajectory of the spiral. (G) Timeseries of the tip speed (red) and curvature of the spiral tip trajectory (black), as the spiral drifts along the LI gradient (green band) or against it (gray band). The profiles correspond to the part of the trajectory shown in the inset of panel D. (H) Increase in the maximum horizontal displacement (d) of the spiral core, with increase in the applied LI gradient (in $\mathrm{mW} / \mathrm{mm}^{3}$ ), within the given time frame $(2 \mathrm{~s}$ ) of the simulation (displacement reported as mean $\pm \mathrm{SD}$, $N=10)$.

The online version of this article includes the following figure supplement(s) for figure 2:

Figure supplement 1. Spatial drift of a spiral wave imposed by a exponential pattern of sub-threshold illumination.

direction of the illuminated region, the drift velocity decreases exponentially with time. Once the spiral has entered the illuminated region, it settles to a stationary state. This is due to the homogeneity of the domain away from the interface. Figure 3B demonstrates the spatiotemporal response of the membrane voltage to the applied light pattern in a quiescent domain (no spiral wave), along the $\mathrm{x}$-axis, at $\mathrm{y}=0.75 \mathrm{~cm}$ on the $2 \mathrm{D}$ domain, perpendicular to the illumination pattern, shown as a dashed-dot line in Figure 3A. It shows that the inhomogeneity of the domain at the interface causes a spiral wave to drift, but this drift is eventually inhibited as the spiral migrates away from the interface. The corresponding evolution of the magnitude of the spatial derivative of $V$ is illustrated in 


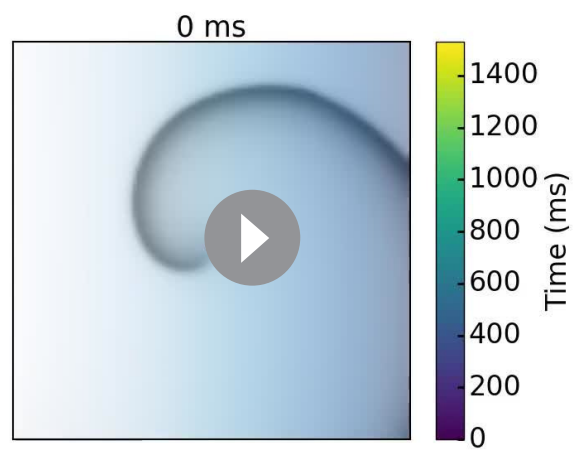

Video 1. Spatial drift of the spiral wave imposed by an LI gradient of $8 \times 10^{4} \mathrm{~mW} / \mathrm{mm}^{3}$. The spiral wave drifts along the illumination gradient direction. Finally the spiral wave collides to the boundary and is terminated. https://elifesciences.org/articles/59954\#video 1
Figure 3C, with detailed analyses into the temporal growth of the peak $|d V / d x|$ and width of the $|d V / d x|$ profile at $10 \%$ maximum height (Figure 3C, inset). A comparison of the peak $|d V / d x|$ in Figure $2 C$ and $F$ and $3 C-D$, shows that $|d V / d x|_{\max }$ in Figure 3 is an order of magnitude larger than those in Figure 2. This large value of $|d V / d x|_{\max }$ at the interface leads to a rapid drift of the spiral wave in the initial phase, followed by gradual deceleration (Figure 3D). At high LI, the tip speed shows large oscillations as the spiral moves along or against the voltage gradient imposed by the illumination. These oscillations are restricted to the width of the interface, which correlates with the width of $|d V / d x|$ at $10 \%$ peak height. However, once the spiral wave enters the illuminated region, its tip speed begins to decrease (Figure 3D) until it reaches a constant value. Inset of Figure 3D illustrates the mean squared displacement of the spiral wave tip during first 800 ms shown as shaded gray region in the speed plot. Typically, the drift velocity of the spiral tip in the initial phase is proportional to the slope of the gradient at the interface. However, as the spiral migrates in the direction of the illuminated region, the drift velocity decreases exponentially with time. Once the spiral has entered the illuminated region, it settles to a stationary state. We observe that the time required by the spiral to reach this state also depends on the intensity of the applied light. Thus, for the cases of $\mathrm{LI}>0.015$ $\mathrm{mW} / \mathrm{mm}^{2}$, the spiral wave settles to zero drift velocity (stationary state) within $2 \mathrm{~s}$ of observation time, whereas in others with lower LI, the drift velocity decreases to a small non-zero constant value, within the $2 \mathrm{~s}$ of observation time, considered. We calculated the drift velocity, for each $\mathrm{LI}$ and found that it decays exponentially with time as the spiral transits from the interface toward the illuminated region (Figure 3E). We used a function (Equation1) to fit the instantaneous drift velocity as a function of time:

$$
V(t)=V_{0} e^{(-t / \tau)}
$$

We found that by increasing $\mathrm{LI}$ from 0.001 to $0.015 \mathrm{~mW} / \mathrm{mm}^{2}$, the initial velocity $V_{0}$ increases by factor of 20 and it decreases faster as manifested in the calculated values of the decay constant $(\tau)$ (Table 1). At $\approx 0.6 \mathrm{~cm}$ from the interface, the influence of $d V / d x$ becomes so negligible, that the spiral establishes a stable core, bounded by a circular trajectory of its tip. A measurement of the net horizontal displacement $(d)$ of the spiral tip from its initial location, at different $\mathrm{LI}$ during $2 \mathrm{~s}$ of simulation shows that $d$ increases only slightly with increase in $\mathrm{LI}$ (Figure 3F). To understand the basis for the flatenning of the $d$-LI curve, we cross-checked the mean displacements at each LI with our data on drift speed. In order to do so, we integrated the exponential fit of the instantaneous drift speed, over the time required by the spiral wave to attain stationarity, and found that the calculated displacement falls in the range of $0 \%$ to $22 \%$ tolerance of the measured numbers for $d$ in 
A
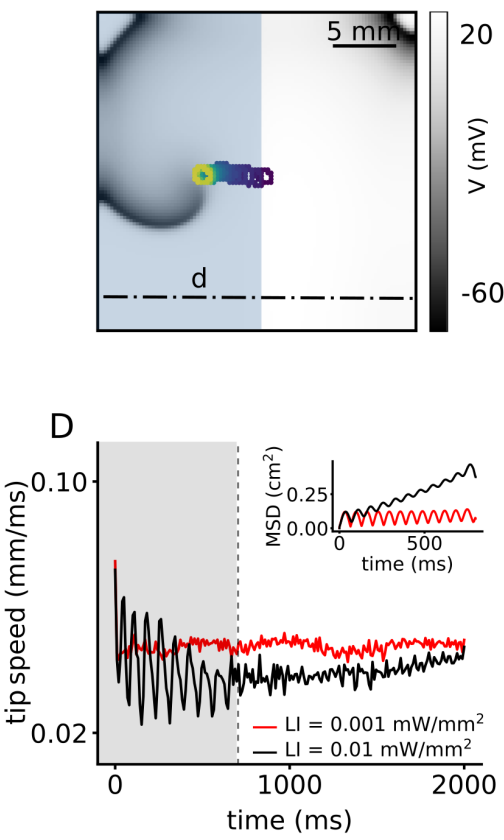

B
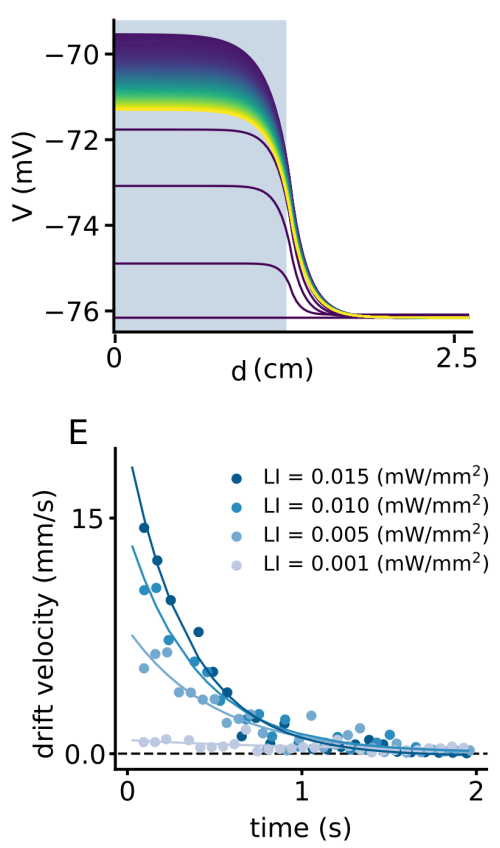

C
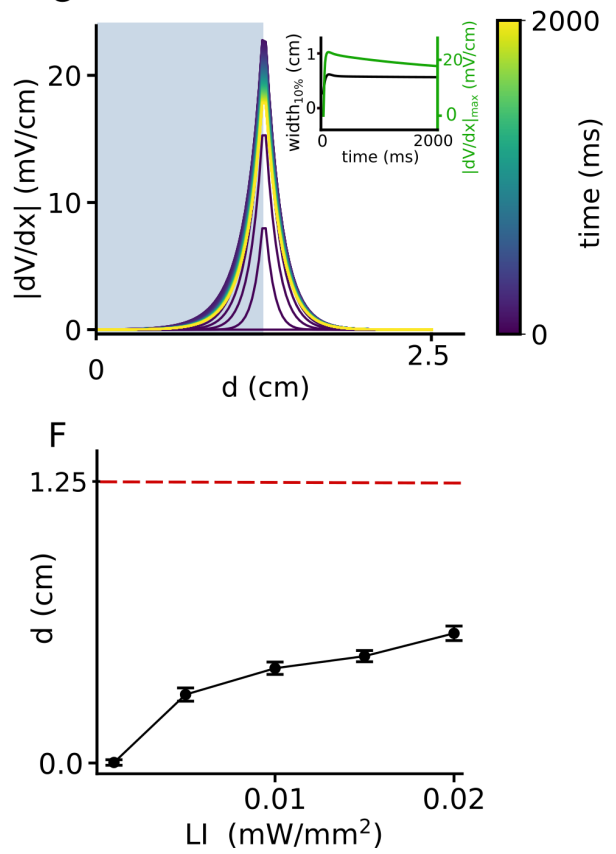

Figure 3. Spatial drift of a spiral wave in a domain that is partially illuminated with sub-threshold LI. (A) Trajectory of the spiral wave tip, as it drifts from the non-illuminated region to the region illuminated with $\mathrm{LI}=0.01 \mathrm{~mW} / \mathrm{mm}^{2}$. Colors indicate different times, here and $\mathrm{B}$ - $\mathrm{C}$. (B) Time evolution of the voltage distribution along the dashed line indicated in (A), in a quiescent domain with the same illumination. (C) Spatial derivative of $V(d V / d x)$ along the dashed line in (A), at different times, for the same illumination as in (A-B). Inset shows the time evolution of the distribution of $d V / d x$ at the interface between the illuminated and non-illuminated regions. The green curve shows the timeseries of the peak $d V / d x$, whereas the black curve shows the corresponding timeseries for the width of the distribution in (C). We defined 'width' as the horizontal distance between two points in the domain where $\left|\frac{d V}{d x}\right|=0.1\left|\frac{d V}{d x}\right|_{\max }$. (D) Timeseries of the spiral tip speed at LI $=0.001 \mathrm{~mW} / \mathrm{mm}^{2}$ (red) and $0.01 \mathrm{~mW} / \mathrm{mm}^{2}$ (black). Inset shows the mean square displacement profiles corresponding to the first $800 \mathrm{~ms}$ of illumination, shaded gray in the speed plot. (E) Timeseries of the drift speed of the spiral core at $\mathrm{LI}=0.001$ (gray), 0.005 (light blue), 0.01 (dark blue), and $0.015 \mathrm{~mW} / \mathrm{mm}^{2}$ (indigo). We observe that for any LI, drift speed decreases exponentially with time as the spiral core crosses the interface. (F) Slow increase in the maximum horizontal displacement (d) of the spiral core, with increase in LI applied to one half of the domain (displacement reported as mean $\pm S D, N=10$ ).

Figure 3F. These values are presented in Table 1. A study of the trends of the exponential fits presented in Figure 3E shows that the higher the LI, the faster is the drift velocity across the interface. However, once the width of the interface has been crossed, drift velocity decreases rapidly to zero. Thus, the net displacement of the spiral core during the total time is comparable for different $\mathrm{LI}$ at high sub-threshold intensities.

To summarize, our results indicate that with a single step-like illumination gradient, even at the highest LI considered, the spiral wave does not drift sufficiently to collide with the boundary and

Table 1. Comparison between calculated drift-induced displacement of the spiral wave (calculated d), and the observed maximum displacement (d) at different $\mathrm{LI}$, for the single-step illumination pattern.

\begin{tabular}{llllll} 
니 $\left(\mathrm{mW} / \mathrm{mm}^{2}\right)$ & $V_{0}(\mathrm{~mm} / \mathrm{s})$ & $\tau(\mathrm{s})$ & Calculated $\mathbf{d}(\mathrm{cm})$ & $D(\mathrm{~cm})$ & Tolerance $(\%)$ \\
\hline 0.001 & 1 & 1.1 & 0.09 & 0.09 & 0 \\
\hline 0.005 & 8 & 0.49 & 0.375 & 0.39 & 4 \\
\hline 0.010 & 14 & 0.39 & 0.477 & 0.53 & 11 \\
\hline 0.015 & 20 & 0.35 & 0.55 & 0.66 & 22 \\
\hline
\end{tabular}


Table 2. Experimental study of the intact mouse heart to study the arrhythmia frequency.

\begin{tabular}{|c|c|c|c|}
\hline Reagent type & Designation & Source & Additional information \\
\hline Biological sample & $\begin{array}{l}\text { Transgenic mouse heart } \\
\text { expressing ChR2 }\end{array}$ & $\begin{array}{l}\text { Dr. S. Sonntag, PolyGene AG, } \\
\text { Switzerland }\end{array}$ & Isonated form transgenic mouse ( $\alpha-M H C-C h R 2)$ \\
\hline $\begin{array}{l}\text { Chemical compound, } \\
\text { drug }\end{array}$ & Di-4-ANBDQPQ stain & AAT Bioquest & $\begin{array}{l}\text { Red-shifted voltage-sensitive dye to optically probe } \\
\text { membrane potentials }\end{array}$ \\
\hline Software, algorithm & AcqKnowledge & BIOPAC Systems, Inc & Software for Data Acquisition and Analysis \\
\hline
\end{tabular}

terminate itself. Therefore, stepwise illumination is attractive enough to draw the spiral into an illuminated area, but stabilization of the core occurs afterwards.

Our results with a single step-like gradient of illumination suggest that we need not one but a sequence of such steps to attract a spiral wave and drag it toward an inexcitable boundary to ensure its termination. Thus, we apply the following modification to the current protocol: Once the spiral wave enters an illuminated region, as in the case of half-domain illumination, we adjust the position of the interface to further pull the spiral toward an inexcitable domain boundary, resulting in continuous drift of the core. To this end, we decrease the size of the illuminated region in three steps, from half $\left(1.25 \times 2.5 \mathrm{~cm}^{2}\right)$ to a twentieth $\left(0.125 \times 2.5 \mathrm{~cm}^{2}\right)$ of the domain size, with a spatial interval of $0.375 \mathrm{~cm}$. At each step, we apply uniform illumination with a constant pulse width. Figure 4A illustrates drift-induced termination of a spiral wave at $\mathrm{LI}=0.01 \mathrm{~mW} / \mathrm{mm}^{2}$ and $500 \mathrm{~ms}$ pulse width (see Video 3). Figure 4B demonstrates the spatiotemporal response of the membrane voltage to the applied light pattern in a quiescent domain (no spiral wave), along the $x$-axis, at $y=0.75 \mathrm{~cm}$ on the 2D domain, perpendicular to the illumination pattern during each step, shown as a dashed-dot line in Figure 4A. As the illuminated area is reduced, the sharp peak in $d V / d x$ at the interface between illuminated and non-illuminated regions shifts toward the boundary (Figure 4B). In consonance with our predictions, the spiral drifts continuously toward the boundary and terminates itself in the process. Figure $4 C$ shows the maximum horizontal displacement $d$ of the spiral wave core, as it is subjected to the multi-step illumination protocol, using regional sub-threshold illumination with $\mathrm{LI}=0.01$, and $0.02 \mathrm{~mW} / \mathrm{mm}^{2}$, respectively, and a range of pulse lengths (PL) varying from 50 to $1000 \mathrm{~ms}$. Figure 4D shows the dependence of spiral termination time on PL, for the two chosen LI, in the cases where successful termination did occur.

Finally, to compare the efficiency of spiral wave termination from different illumination protocols, such as illumination with a smooth gradient in light intensity (LI) from 0.0 to $0.01 \mathrm{~mW} / \mathrm{mm}^{2}$ (Figure 2A) and multi-step adjusted pattern illumination with constant $\mathrm{LI}=0.01 \mathrm{~mW} / \mathrm{mm}^{2}$ (Figure 4A), we calculated the mean square displacement (MSD) of the spiral wave core during $2 \mathrm{~s}$ of illumination (Figure 4E). Our studies showed that the MSD for the case of gradient illumination (shown as an orange curve in Figure 4E) was $\approx 0.25 \mathrm{~cm}^{2}$ at the end of $2 \mathrm{~s}$, which was far from the boundary, whereas, that in the case of multi-step adjusted pattern illumination (shown as a green curve in Figure 4E), was $1.25 \mathrm{~cm}^{2}$. The drift velocity $(V(t))$ of the spiral wave core in each case, is shown in Figure 4F. These results indicate that $V(t)$ is $\simeq 4 x$ higher in case of illumination via multistep adjusted pattern, than that measured with gradient illumination. Each minimum value for the case of a multi-step pattern illumination corresponds to the time at which the illumination pattern is set to the next step. Such adjustment causes the spiral wave to drift continuously toward the boundary, whereupon it terminates within $2 \mathrm{~s}$ of simulation. Thus, the multi-step adjusted pattern proves to

Table 3. Experimental study of the intact mouse heart to study the conduction velocity.

\begin{tabular}{|c|c|c|c|}
\hline Reagent type & Designation & Source & Additional information \\
\hline $\begin{array}{l}\text { Biological } \\
\text { sample }\end{array}$ & $\begin{array}{l}\text { Transgenic mouse heart expressing } \\
\text { ChR2 }\end{array}$ & Prof. Marina Campione, University of Padova, Italy & $\begin{array}{l}\text { Isolated from transgenic mouse (ChR2- } \\
\text { MyHC6-Cre+) }\end{array}$ \\
\hline $\begin{array}{l}\text { Chemical } \\
\text { compound, } \\
\text { drug }\end{array}$ & Di-4-ANBDQPQ stain & $\begin{array}{l}\text { Prof. Leslie M. Loew, Center for Cell Analysis and } \\
\text { Modeling, UConn Health, Farmington (USA) }\end{array}$ & $\begin{array}{l}\text { Red-shifted voltage-sensitive dye to } \\
\text { optically probe membrane potentials }\end{array}$ \\
\hline $\begin{array}{l}\text { Software, } \\
\text { algorithm }\end{array}$ & $\begin{array}{l}\text { LabVIEW } 2015 \text { (64-bit) software } \\
\text { HCImageLive software camera }\end{array}$ & $\begin{array}{l}\text { National Instruments, Austin, TX, USA Hamamatsu, } \\
\text { Shizuoka, Japan }\end{array}$ & - \\
\hline
\end{tabular}


A

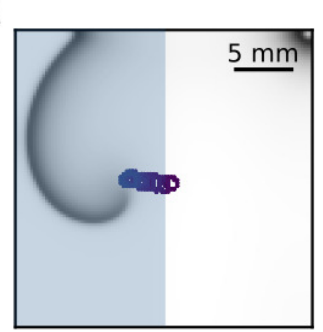

B
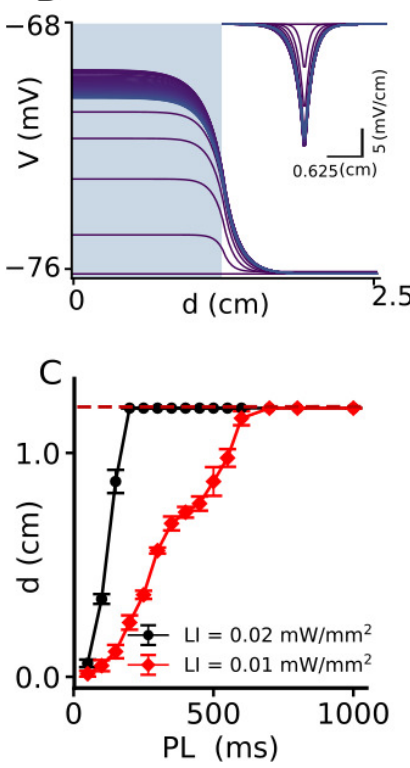
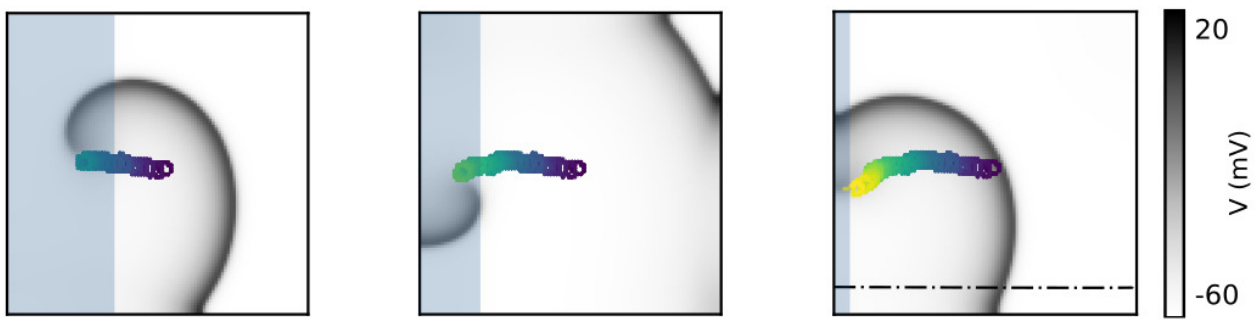

20

छ

$-60$
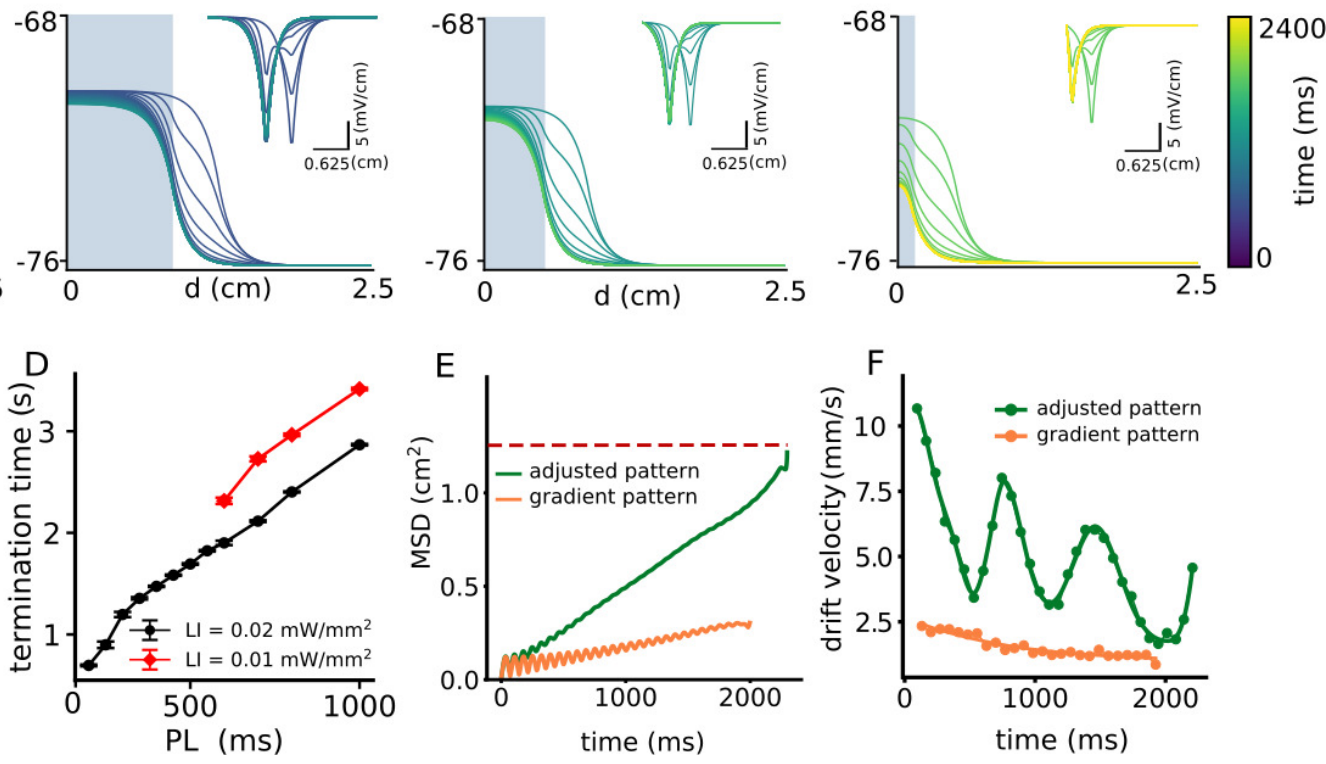

Figure 4. Continuous spatial drift of a spiral wave using a multi-step adjusted pattern illumination protocol. (A) Trajectory of the spiral wave tip, during different steps of the illumination protocol. (B) Time evolution of the voltage distribution and its spatial derivative $d V / d x$ (inset) for each step of the protocol, as measured along the dot-dashed line shown in the last sub-figure of panel (A). (C) Horizontal displacement $d$ of the spiral wave core at $\mathrm{LI}=0.01$ (red) and 0.02 (black) $\mathrm{mmW} / \mathrm{mm}^{2}$, respectively, at different pulse lengths (PL) (displacement reported as mean $\pm \mathrm{SD}, \mathrm{N}=10$ ). ( $\mathrm{D}$ ) Termination time for the cases in which the spiral drifted all the way to the boundary and annihilated itself through collision. Red and black curves represent data for $\mathrm{LI}=0.01 \mathrm{~mW} / \mathrm{mm}^{2}$, and 0.02 (black) $\mathrm{mW} / \mathrm{mm}^{2}$, respectively (termination time reported as mean $\pm \mathrm{SD}, \mathrm{N}=10$ ). (E) Mean squared displacement of the spiral wave core with two different illumination protocols: multi-step adjusted pattern (green) and gradient pattern (orange). (F) Drift velocity of the spiral wave core for each case of illumination patterns, multi-step adjusted pattern (green) and gradient pattern (orange).

be faster at effectuating drift-induced termination of spiral waves, than the gradient illumination pattern.

\section{Discussion}

Two major factors responsible for the induction of spiral wave drift in cardiac tissue, are (i) intrinsic tissue heterogeneity (Kharche et al., 2015) and (ii) perturbation by an external force (Wellner et al., 2010; Biktashev et al., 2011). In the first case, the heterogeneity of the tissue may impose a gradient of refractoriness, or result in a non-stationary refractory period of the spiral wave, which would force the spiral to drift (Krinski, 1968; Ermankova and Pertsov, 1988). Heterogeneity in cardiac tissue can occur in two forms: in structure and in function. Structure-induced drift of spiral waves was studied by Kharche et al., 2015 and Woo et al., 2008, among others. They found that the anatomy of the heart, along with differences in cell structure, is responsible for the induction of drift. However, spiral wave drift can also occur because of functional heterogeneities resulting from dispersion 


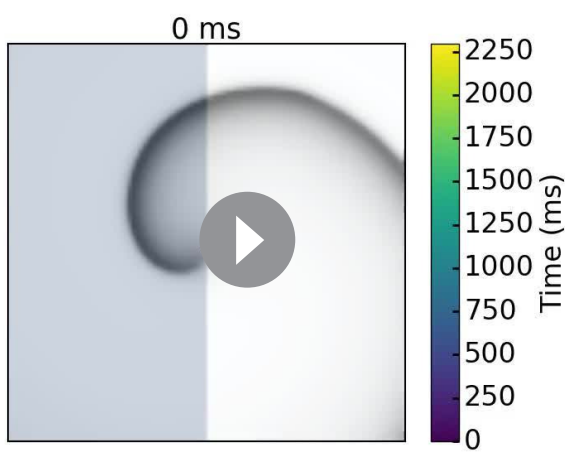

Video 3. Continuous spatial drift of a spiral wave using a multi-step adjusted pattern illumination with $\mathrm{LI}$ of $0.01 \mathrm{~mW} / \mathrm{mm}^{2}$. At each step of reducing the size of the illuminated region, the illumination was applied with a constant illumination PL of $600 \mathrm{~ms}$. The spiral wave drifts continuously along with the reduction direction of the illuminated region size. Finally it collides to the boundary and is terminated.

https://elifesciences.org/articles/59954\#video3 of electrophysiological parameters such as APD and $\mathrm{CV}$ within the tissue. This phenomenon is, in fact, more common.

Regardless of the origin of such functional heterogeneity, Biktasheva et al., 2010 studied its effect on the dynamics of spiral waves in generic FitzHugh-Nagumo and Barkely models, with a stepwise distribution of heterogeneity, similar to what we used in our study (Figure 3). They showed that the center of rotation of the spiral wave can move towards one side of the step and then gradually freeze over time or continue to drift along the step with constant velocity. Our current study, which considers a realistic ionic model with 44 dynamical variables, shows similar dynamical behavior. In our case, the drift velocity is not constant. The spatial profile of the lightinduced voltage gradient allows the spiral to drift with high speed while crossing the interface (see Figure 3E). However, as the spiral leaves the interface, the drift velocity gradually decreases until it reaches a very small positive value at large distances from the interface within the time frame of our simulations.

Of particular interest is the drift velocity trend for different $\mathrm{LI}$ and $\nabla \mathrm{LI}$. We observe that the spiral wave drifts more slowly at small values of $\nabla \mathrm{LI}$, compared to large $\nabla \mathrm{LI}$ (Figure $2 \mathrm{H}$ ). This can be explained by studying the general dynamic behaviour of the spiral wave at different LI. Figure 1D shows that at small LI $\left(<0.01 \mathrm{~mW} / \mathrm{mm}^{2}\right)$, the properties of the spiral core (e.g. $d_{\text {core }}$ ) are unaffected by the applied illumination. Thus, the application of a light gradient at small $\nabla \mathrm{LI}\left(<4 \times 10^{4} \mathrm{~mW} / \mathrm{mm}^{3}\right.$ in Figure $\left.2 \mathrm{H}\right)$, has a negligible effect on the dynamics of the spiral, resulting in very slow drift. On the contrary, at $\mathrm{LI}>0.01 \mathrm{~mW} / \mathrm{mm}^{2}$ (Figure 1D), $d_{\text {core }}$ increases rapidly, leading to a strong decrease of the rotation frequency of the spiral wave. This means that the spiral now needs a little more time $(\tau)$ to complete a single cycle of its rotation, before it can move one step $\left(L_{\text {step }}\right)$ in space. It should be noted, however, that the rapid increase of $d_{\text {core }}$ causes a corresponding increase of $L_{\text {step }}$, which more than compensates for the increase of $\tau$. So the drift velocity effectively increases.

If, on the other hand, the case is considered with a single step of illumination (Figure 3F), the maximum displacement $(d)$ of the spiral wave core saturates with increase of LI. This can be explained by looking at the spatiotemporal distribution of $d V / d x$ at the interface between the illuminated and non-illuminated regions. Our studies show that when light is applied to one half of the domain, a spatial profile of $|d V / d x|$ is established, which, over time, evolves such that the peak height decreases and width increases to saturation values. Both $\left|\frac{d V}{d x}\right|_{\max }$ and width of $|d V / d x|$, at the instant of first illumination, increases with the LI. The gradual spatiotemporal evolution of the $|d V / d x|$ profile ensures nonlinearity in the drift velocity, which shows an exponential decrease over time. Once the spiral leaves the zone of influence of the interface, drift either stops, or becomes constant and occurs in the direction parallel to the interface. The net displacement of the spiral core can be calculated by integrating the drift velocity over time as the spiral crosses the interface. Drift velocity for high $\mathrm{LI}\left(v_{\text {drift, } \sim \text { high }}\right)$ is initially larger than that for low LI $\left(v_{\text {drift, low }}\right)$. However, because of the nature of the spatial profile of $|d V / d x|,\left(v_{d r i f t, \sim h i g h}\right)$ decreases at a rate that is much faster than $\left(v_{d r i f t, \sim l o w}\right)$, such that, $\left(v_{\text {drift }, \sim \text { high }}\right)$ drops to zero sooner than $\left(v_{\text {drift, low }}\right)$. Consequently, the corresponding displacement of the spiral core in the different cases with high LI come comparable. Hence the flatenning of the curve in Figure 3F.

In our simulations, we observed that the effective drift direction of a spiral wave always follows the direction of increasing light intensity. This is consistent with the findings of Davydov et al., 1988. Similar observations were also made by Markus et alı, 1992, in light-sensitive Belousov- 
Zhabotinsky (BZ) reactions, where they used experiments to demonstrate positive phototaxis of a spiral wave, in the presence of a gradient of illumination. In our study we use this feature strategically to remove spiral waves from the domain by drift-induced collision with the boundary in favor of termination.

While previous studies by Feola et al., 2017 and Majumder et al., 2018 have proven that full spatio-temporal control over the dynamics of a spiral wave can be achieved by manipulating its core with supra-threshold illumination, the efficacy of their respective methods at sub-threshold illumination, remained untested. In this study, we exploited the power of regional sub-threshold illumination, to manipulate spiral wave dynamics with or without prior knowledge about the location of the spiral core.

Spiral wave drift can be investigated by many different approaches; each approach has its advantages and disadvantages and is designed based on the specific parameters of the system. The general conclusion is that controlled drift can lead to effective termination of spiral waves. It is therefore important to have efficient control over spiral wave drift. To this end, it is essential to develop a deeper understanding of the dynamics of drift. It has been established so far, that light-sensitive BZ reaction is the easiest-to-control excitable system for the study of spiral wave drift in experiments. Thus, optogenetics, which is the analogous tool for light-sensitivity in cardiac tissue, is expected to hold great potential in studies that involve exercising control over spiral wave dynamics in the heart (Braune and Engel, 1993). Our results prove the validity of this statement by demonstrating the use of optogenics to study and control spiral wave drift in 2D cardiac tissue.

Finally, controlled spiral wave drift finds its main application in optogenetics defibrillation. Current techniques for such defibrillation use global or structured illumination patterns applied to the epicardial surface of the heart. Due to the poor penetration of light into cardiac tissue, most of the applied light is scattered or absorbed before it can reach the endocardium. This is considered a major limitation of optogenetics, as the applied supra-threshold illumination cannot be expected to affect subsurface electrical activity in the heart wall. However, ex vivo studies on small mammalian hearts consistently demonstrate the success of optogenetic defibrillation, without providing a clear mechanism for the same (Bruegmann et al., 2016). Some studies try to explain the mechanism behind this success, using the critical mass hypothesis (Zipes et alı, 1975). According to this hypothesis, a spiral (scroll) wave requires a minimum area (volume) of excitable tissue for its sustainment. By applying supra-threshold light to the surface of the epicardium, one can effectively reduce available area (volume) of excitable tissue to below the threshold requirement for spiral sustainability, thereby forcing the wavefront of the spiral (scroll) wave to collide with its waveback, resulting in its termination. However, our studies postulate an alternative theory. We propose that the application of light to the epicardial surface effectively leads to a transmural illumination gradient within the heart wall, with both sub-threshold and supra-threshold illumination régimes. Our study shows that a linear gradient of pure sub-threshold illumination has the potential to induce a drift of a spiral wave into the region of higher illumination (i.e. the epicardial surface in a transmural section of the heart wall), thereby protecting the internal tissue from hidden elec-

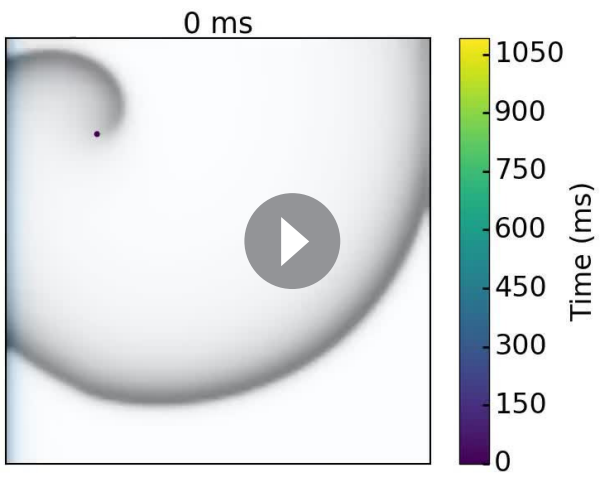

Video 4. Drift of a spiral toward the illuminated region with an exponential decay pattern with $\mathrm{LI} 0.07 \mathrm{~mW} / \mathrm{mm}^{2}$. https://elifesciences.org/articles/59954\#video4 trical activity. Once such activity is drawn out to the surface by the induced drift, it can be terminated using global supra-threshold illumination, which then ensures electrical synchronization.

Thus, our study provides new mechanistic insights into the theory of successful optogenetic defibrillation in animal hearts, where application of light to the surface produces a gradient of illumination within the heart wall. This gradient is typically exponential. However, to a first approximation, we have treated it as a linear gradient to study the effect of subthreshold illumination in a simple system. Saito et al., 2017 shows the approximate space constant for exponential decay of blue light in cardiac tissue is $0.6 \mathrm{~mm}$. Studies by Bruegmann et al., 2016 demonstrate 
attenuation of light at a depth of $1 \mathrm{~mm}$ beneath the surface of the cardiac tissue, which limits the effect of supra-threshold illumination to only a few layers below the surface. Video 4 shows drift of the spiral wave toward the illuminated region when the wave is located at the neighboring of the illumination gradient with a exponential decay pattern of illumination in the cardiac tissue. Figure 2figure supplement $1 A$ and $B$ show time evolution of the voltage and spatial derivative of the voltage in a quiescent domain, respectively. Video 5 shows a spiral wave rotation in a circular trajectory where there no illumination pattern. This hypothesis can be also extended to the conventional defibrillation methods. Applying an electric field to excite the heart tissue results in the development of a transmural depolarization gradient (Dosdall et al., 2010). The functional heterogeneity caused by these depolarization gradients may force spiral waves to drift. Such a drift occurs in the direction of the positive gradient, resulting in the emergence of the spiral cores on the surface, where they are eliminated through synchronisation.

We propose to test this hypothesis in cell culture experiments by projecting a gradient subthreshold light pattern on a monolayer of optogenetically modified mouse cardiac cells. Such a pattern can be easily generated by using a diffuse light source that is slightly translated perpendicular to the field of view of the microscope. As a next step, one can try illuminating the epicardium in a transmural slice of the mouse heart using a ring of LEDs (so that the slice is illuminated uniformly, along the periphery). Using supra-threshold light on the surface of the epicardium, one can observe the dynamics of the spiral wave as in the presence of the gradient sub-surface heterogeneity. This hypothesis can be also extended to the conventional defibrillation methods. Applying an electric field to excite the heart tissue results in the development of a transmural depolarization gradient (Dosdall et al., 2010). The functional heterogeneity caused by these depolarization gradients may force spiral waves to drift. Such a drift occurs in the direction of the positive gradient, resulting in the emergence of the spiral cores on the surface, where they are eliminated through synchronisation.

\section{Materials and methods}

\section{Numerical study}

Electrical activity in single cardiac cells was modeled according to Equation 2. Here, $V$ is the transmembrane voltage that arises from ionic gradients that develop across the cell membranes.

$$
\frac{d V}{d t}=-\frac{I_{\text {ion }}+I_{\text {stim }}}{C_{m}}
$$

The total ionic current $I_{\text {ion, }}$ flowing across the membrane of a single cell, was mathematically described using the electrophysiological model of an adult mouse ventricular cardiomyocyte, first introduced by Bondarenko et alı, 2004, including the model improvements in PetkovaKirova et al., 2012. The model contains of 40 dynamical variables solved by a fourth-order RungeKutta method with the temporal resolution of $10^{-4} \mathrm{~ms}$. Solving these variables describes 15

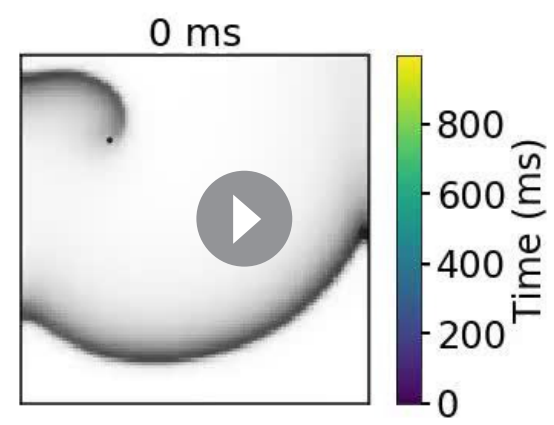

Video 5. A spiral wave rotation with no illumination pattern.

https://elifesciences.org/articles/59954\#video5 different currents as per Equation 3.

$$
\left.\left.I_{i o n}=I_{N a}+I_{C a L}+I_{p C a}+I_{K t o, f}+I_{K t o, s}+I_{K r}+I_{K u r}+I_{K B}\right\}\right)+I_{K \% 1}+
$$

Here, $I_{N a}$ is the fast $\mathrm{Na}^{+}$current, $I_{C a L}$ is the L-type $\mathrm{Ca}^{2+}$ current, $\mathrm{I}_{p \mathrm{Ca}}$ is the $\mathrm{Ca}^{2+}$ pump current, $I_{K t o, f}$ is the rapidly recovering transient outward $K^{+}$current, $I_{K t o, s}$ is the slowly recovering transient outward $K^{+}$current, $I_{K r}$ is the rapid delayed rectifier $K^{+}$current, $I_{K u r}$ is the ultrarapidly activating delayed rectifier $K^{+}$current, $I_{K s s}$ is the non-inactivating steady-state voltageactivated $K^{+}$current, $I_{K 1}$ is the time-independent inwardly rectifying $K^{+}$current, $I_{K s}$ is the slow delayed rectifier $\mathrm{K}^{+}$current, $\mathrm{I}_{\mathrm{NaCa}}$ is the $\mathrm{Na}^{+}$/ 
$\mathrm{Ca}^{2+}$ exchange current, $\mathrm{I}_{\mathrm{NaK}}$ is the $\mathrm{Na}^{+} / \mathrm{K}^{+}$pump current, $\mathrm{I}_{\mathrm{Cl}, \mathrm{Ca}}$ is the $\mathrm{Ca}^{2+}$-activated $\mathrm{Cl}^{-}$current , $\mathrm{I}_{\mathrm{Cab}}$ is the background $\mathrm{Ca}^{2+}$ current and $I_{\mathrm{Nab}}$ is the background $\mathrm{Na}^{+}$current.

In spatially extended media, such as $2 \mathrm{D}$, cardiac cells communicate with each other through intercellular coupling. The membrane voltage is then modeled using a reaction-diffusion type equation (Equation 4):

$$
\frac{d V}{d t}=\nabla(D \nabla V)-\frac{I_{i o n}+I_{\text {stim }}}{C_{m}}
$$

The first term on the right side of the equation shows the intercellular coupling. $D$ is the diffusion tensor, which is assumed here to be a scalar and has the value $0.00014 \mathrm{~cm} / \mathrm{ms}$. In this 2D monodomain model, the excitation wave propagates with an isotropic conduction velocity of $43.9 \mathrm{~cm} / \mathrm{s}$. This simulation domain consists of $100 \times 10$ or $100 \times 100$ grid points. We use a spatial resolution of $0.025 \mathrm{~cm}$ and time step $10^{-4} \mathrm{~ms}$. We apply no-flux boundary conditions at the inexcitable domain boundaries.

To create a spiral wave in the domain, we first selected four sets of values of the model parameters corresponding to four different phases of the AP: resting state, depolarized state and two states of repolarization (one at the beginning and one towards the end) (see Figure 1-figure supplement 1A). Next, we partitioned the domain into four sections and initialized all the dynamical variables with the values selected in the previous step (see Figure 1-figure supplement 1B). In clockwise direction, starting from the top left corner, we initialized the domain sections with values corresponding to the resting state, the depolarized state, beginning of the repolarization state and end of the repolarization state, respectively. A plane wave then begins to propagate from the upper right to the upper left quarter of the domain. Since the lower half of the domain is refractory, the wave cannot propagate into it. However, with time, the lower left quarter recovers completely, allowing the wave to curl into it from above. Finally, the lower right quarter recovers for excitation and allows wave propagation into it, thereby completing the circuit for the spiral to travel through. Figure 1-figure supplement 1D shows a series of frames during the process of spiral wave formation.

To include light sensitivity, the model is coupled to the mathematical model of a light-activated protein called channelrhodopsin-2 (ChR2) (Williams et al., 2013). This protein is a non-selective cation channel that reacts to blue light with a wavelength of $470 \mathrm{~nm}$. The inward ChR2 current $\left(I_{C h R 2}\right)$ is mathematically described by the following equation:

$$
I_{C h R 2}=g_{C h R 2} G(V)\left(O_{1}+\gamma O_{2}\right)\left(V-E_{C h R 2}\right)
$$

Here, $g_{C h R 2}$ is the conductance, $G(V)$ is the voltage rectification function, $\mathrm{O}_{1}$ and $\mathrm{O}_{2}$ are the open state probabilities of the ChR2, $\gamma$ is the ratio $O_{1} / O_{2}$, and $E_{C h R 2}$ is the reversal potential of this channel. By including the mathematical model of the ChR2 to this model, we can stimulate the system optically at the single cell level or the 2D monodomain level. In our studies, a stationary spiral had a circular core with constant curvature. Over time, the induction of drift led to a change in the curvature of the tip trajectory. The instantaneous curvature $(k)$ of the spiral tip trajectory was calculated according to Equation 6, where $(x, y)$ represents the coordinate of each point of the trajectory. For studies on spiral wave drift in the presence of gradient and stepwise illumination, we calculated maximum displacement $d$ at the end of $2 \mathrm{~s}$, as mean of 10 different initial conditions of the spiral.

$$
k=\frac{x^{\prime} y^{\prime \prime}-y^{\prime} x^{\prime \prime}}{\left(x^{\prime 2}+y^{\prime 2}\right)^{\frac{3}{2}}}
$$

\section{Experimental study}

All experiments in the intact mouse heart were done in accordance with the guidelines from Directive 2010/63/EU of the European Parliament on the protection of animals used for scientific purposes and the current version of the German animal welfare law and were reported to our animal welfare representatives. The experimental protocol was approved by the Italian Ministry of Health; authorization $n^{\circ}$ 944/2018 $\mathrm{P}$ and the responsible animal welfare authority (Lower Saxony State Office for Consumer protection and Food Safety). Humane welfare-oriented procedures were carried out in 
accordance with the Guide for the Care and Use of Laboratory Animals and done after recommendations of the Federation of Laboratory Animal Science Associations (FELASA).

\section{Experimental measurements of arrhythmia frequency in the intact mouse heart}

To observe the effects of sub-threshold illumination on the arrhythmia frequency, we applied light globally to the hearts of Langendorff-perfused adult $\alpha-M H C-C h R 2$ transgenic mice (Quiñonez Uribe et al., 2018). The expression of channelrhodopsin-2 (ChR2) in these hearts was restricted to cardiomyocytes. For perfusion, we used the standard protocol of retrograde Langendorff perfusion with tyrode solution (130 mM NaCl, $4 \mathrm{mM} \mathrm{KCl}, 1 \mathrm{mM} \mathrm{MgCl} 2,24 \mathrm{mM} \mathrm{NaHCO}, 1.8 \mathrm{mM} \mathrm{CaCl}, 1.2 \mathrm{mM}$ $\mathrm{KH}_{2} \mathrm{PO}_{4}, 5.6 \mathrm{mM}$ glucose, $1 \%$ albumin/BSA, aerated with carbogen [95\% oxygen and $5 \% \mathrm{CO}$ ]). All experiments were performed at $37^{\circ} \mathrm{C}$. Arrhythmia was induced by applying 30 electrical pulses (2.3$2.5 \mathrm{~V}$ amplitude), at frequencies of $30-50 \mathrm{~Hz}$, using a needle electrode. To stabilize the arrhythmia, (i) the concentration of $\mathrm{KCl}$ in tyrode solution was reduced from $4 \mathrm{mM}$ to $2 \mathrm{mM}$, and (ii), $100 \mu \mathrm{M}$ Pinacidil, (a KATP channel activator) was added to the tyrode. To exclude the possibility of self-termination, we considered only those cases in which the arrhythmia lasted longer than $5 \mathrm{~s}$. Next, a single blue light pulse $(\lambda=470 \mathrm{~nm}$, pulse duration $=1 \mathrm{~s}$ ) was applied using 3 LEDs positioned at angular separation $120^{\circ}$, around the bath, to provide global illumination. We repeated the experiments for $\mathrm{LI}=0.0011,0.0041,0.0078,0.0124$, and $0.0145 \mathrm{~mW} / \mathrm{mm}^{2}$, respectively, and measured the DF of the arrhythmia using a method of Fourier transform (FT) in those experiments that did not result in the termination of the arrhythmia. We considered data from two hearts with seven experiments on each.

\section{Experimental measurements of conduction velocity in the intact mouse heart}

A wide-field mesoscope operating at a frame rate of $2 \mathrm{kHz}$ (Scardigli et al., 2018) was used to map the action potential propagation in Langendorff horizontally perfused adult mouse hearts expressing ChR2 (under the control of $\alpha-M y H C-C h R 2$ promoter) and stained with a red-shifted voltage sensitive dye (di-4-ANBDQPQ; Matiukas et al., 2007). To observe the effect of sub-threshold ChR2 stimulation on action potential conduction velocity, the heart was uniformly illuminated with blue light during electrical pacing at the heart apex $(5 \mathrm{~Hz})$. Conduction velocity was calculated by measuring the AP propagation time between two regions place at a known distance. The experiments were repeated for four different hearts at the $\mathrm{LI}=0,0.0104,0.0175,0.0262,0.0894,0.1528 \mathrm{~mW} / \mathrm{mm}^{2}$. We considered data from four hearts with three experiments on each.

\section{Acknowledgements}

We thank Dr. Florian Spreckelsen, Babak Vajdi Hokmabad, and all the members of biomedical physics group (bmpg) of Max Planck Institute for Dynamics and Self-Organization (MPIDS) for their fruitful input.

\section{Additional information}

\begin{tabular}{|c|c|c|}
\hline Funder & Grant reference number & Author \\
\hline $\begin{array}{l}\text { German Center for Cardiovas- } \\
\text { cular Research }\end{array}$ & & Stefan Luther \\
\hline German Research Foundation & $\begin{array}{l}\text { SFB } 1002 \text { Modulatory Units } \\
\text { in Heart Failure }\end{array}$ & Stefan Luther \\
\hline Max Planck Society & & Stefan Luther \\
\hline
\end{tabular}

The funders had no role in study design, data collection and interpretation, or the decision to submit the work for publication. 
Author contributions

Sayedeh Hussaini, Conceptualization, Data curation, Software, Formal analysis, Validation, Investigation, Visualization, Methodology, Writing - original draft, Writing - review and editing; Vishalini Venkatesan, Data curation, Investigation, Methodology, Writing - original draft, Writing - review and editing; Valentina Biasci, José M Romero Sepúlveda, Data curation, Validation, Investigation, Visualization, Writing - review and editing; Raul A Quiñonez Uribe, Data curation, Investigation, Methodology; Leonardo Sacconi, Gil Bub, Resources, Software, Supervision, Validation, Investigation, Visualization, Project administration, Writing - review and editing; Claudia Richter, Resources, Investigation, Project administration, Writing - review and editing; Valentin Krinski, Conceptualization, Supervision, Validation, Investigation, Methodology, Writing - review and editing; Ulrich Parlitz, Conceptualization, Supervision, Investigation, Methodology, Writing - review and editing; Rupamanjari Majumder, Conceptualization, Supervision, Validation, Investigation, Writing - original draft, Writing - review and editing; Stefan Luther, Conceptualization, Resources, Software, Supervision, Funding acquisition, Validation, Investigation, Methodology, Writing - original draft, Project administration, Writing - review and editing

\section{Author ORCIDs}

Gil Bub (iD http://orcid.org/0000-0002-5304-0036

Ulrich Parlitz (iD) http://orcid.org/0000-0003-3058-1435

Rupamanjari Majumder (iD http://orcid.org/0000-0002-3851-9225

Stefan Luther (iD https://orcid.org/0000-0001-7214-8125

Ethics

Animal experimentation: All experiments in the intact mouse heart were done in accordance with the guidelines from Directive 2010/63/EU of the European Parliament on the protection of animals used for scientific purposes and the current version of the German animal welfare law and were reported to our animal welfare representatives. The experimental protocol was approved by the Italian Ministry of Health; authorization $n^{\circ} 944 / 2018-P$ and the responsible animal welfare authority (Lower Saxony State Office for Consumer protection and Food Safety). Humane welfare- oriented procedures were carried out in accordance with the Guide for the Care and Use of Laboratory Animals and done after recommendations of the Federation of Laboratory Animal Science Associations (FELASA).

Decision letter and Author response

Decision letter https://doi.org/10.7554/eLife.59954.sa1

Author response https://doi.org/10.7554/eLife.59954.sa2

\section{Additional files}

Supplementary files

- Transparent reporting form

Data availability

All data generated or analysed during this study are included in the manuscript and supporting files.

\section{References}

Ambrosi CM, Ripplinger CM, Efimov IR, Fedorov VV. 2011. Termination of sustained atrial flutter and fibrillation using low-voltage multiple-shock therapy. Heart Rhythm 8:101-108. DOI: https://doi.org/10.1016/j.hrthm.2010. 10.018, PMID: 20969974

Antzelevitch C, Burashnikov A. 2001. Cardiac Arrhythmias: Reentry and Triggered Activity. In: Sperlakis N Kurachi Y, Terzic A, Cohen M. V (Eds). Heart Physiology and Pathophysiology. fourth edition ed. Academic Press. p. 1153-1179. DOI: https://doi.org/10.1016/j.ccep.2010.10.012 
Babbs CF, Tacker WA, VanVleet JF, Bourland JD, Geddes LA. 1980. Therapeutic indices for transchest defibrillator shocks: effective, damaging, and lethal electrical doses. American Heart Journal 99:734-738. DOI: https://doi.org/10.1016/0002-8703(80)90623-7, PMID: 7377095

Biktashev VN, Biktasheva IV, Sarvazyan NA. 2011. Evolution of spiral and scroll waves of excitation in a mathematical model of ischaemic border zone. PLOS ONE 6:e24388. DOI: https://doi.org/10.1371/journal. pone.0024388, PMID: 21935402

Biktasheva IV, Barkley D, Biktashev VN, Foulkes AJ. 2010. Computation of the drift velocity of spiral waves using response functions. Physical Review E 81:066202. DOI: https://doi.org/10.1103/PhysRevE.81.066202, PMID: 20 866496

Bittihn P, Luther G, Bodenschatz E, Krinsky V, Parlitz U, Luther S. 2008. Far field pacing supersedes antitachycardia pacing in a generic model of excitable media. New Journal of Physics 10:103012. DOI: https://doi. org/10.1088/1367-2630/10/10/103012

Bondarenko VE, Szigeti GP, Bett GC, Kim SJ, Rasmusson RL. 2004. Computer model of action potential of mouse ventricular myocytes. American Journal of Physiology-Heart and Circulatory Physiology 287:H1378H1403. DOI: https://doi.org/10.1152/ajpheart.00185.2003, PMID: 15142845

Braune M, Engel H. 1993. Compound rotation of spiral waves in active media with periodically modulated excitability. Chemical Physics Letters 211:534-540. DOI: https://doi.org/10.1016/0009-2614(93)80139-G

Bruegmann T, Malan D, Hesse M, Beiert T, Fuegemann CJ, Fleischmann BK, Sasse P. 2010. Optogenetic control of heart muscle in vitro and in vivo. Nature Methods 7:897-900. DOI: https://doi.org/10.1038/nmeth.1512, PMID: 20881965

Bruegmann T, Boyle PM, Vogt CC, Karathanos TV, Arevalo HJ, Fleischmann BK, Trayanova NA, Sasse P. 2016. Optogenetic defibrillation terminates ventricular arrhythmia in mouse hearts and human simulations. Journal of Clinical Investigation 126:3894-3904. DOI: https://doi.org/10.1172/JCl88950, PMID: 27617859

Burton RA, Klimas A, Ambrosi CM, Tomek J, Corbett A, Entcheva E, Bub G. 2015. Optical control of excitation waves in cardiac tissue. Nature Photonics 9:813-816. DOI: https://doi.org/10.1038/nphoton.2015.196, PMID: 27057206

Crocini C, Ferrantini C, Pavone FS, Sacconi L. 2017. Optogenetics gets to the heart: a guiding light beyond defibrillation. Progress in Biophysics and Molecular Biology 130:132-139. DOI: https://doi.org/10.1016/j. pbiomolbio.2017.05.002, PMID: 28506694

Cysyk J, Tung L. 2008. Electric field perturbations of spiral waves attached to millimeter-size obstacles. Biophysical Journal 94:1533-1541. DOI: https://doi.org/10.1529/biophysj.107.116244, PMID: 17921205

Davidenko JM, Kent PF, Chialvo DR, Michaels DC, Jalife J. 1990. Sustained vortex-like waves in normal isolated ventricular muscle. PNAS 87:8785-8789. DOI: https://doi.org/10.1073/pnas.87.22.8785, PMID: 2247448

Davidenko JM, Pertsov AV, Salomonsz R, Baxter W, Jalife J. 1992. Stationary and drifting spiral waves of excitation in isolated cardiac muscle. Nature 355:349-351. DOI: https://doi.org/10.1038/355349a0, PMID: 1731248

Davydov VA, Zykov VS, Mikhailov AS, Brazhnik PK. 1988. Drift and resonance of helical waves in distributed active media. Radiophysics and Quantum Electronics 31:419-426. DOI: https://doi.org/10.1007/BF01043605

de Ornelas Maia AC, Soares-Filho G, Pereira V, Nardi AE, Silva AC. 2013. Psychiatric disorders and quality of life in patients with implantable cardioverter defibrillators: a systematic review. The Primary Care Companion for CNS Disorders 15:PCC.12r01456. DOI: https://doi.org/10.4088/PCC.12r01456, PMID: 23930235

Deisseroth K. 2011. Optogenetics. Nature Methods 8:26-29. DOI: https://doi.org/10.1038/nmeth.f.324, PMID: 21191368

Dosdall DJ, Fast VG, Ideker RE. 2010. Mechanisms of defibrillation. Annual Review of Biomedical Engineering 12:233-258. DOI: https://doi.org/10.1146/annurev-bioeng-070909-105305, PMID: 20450352

Ermankova EA, Pertsov AM. 1988. Mechanism of the drift of a spiral wave in an inhomogenuous medium. Biofizika 33:338-342.

Fenton FH, Luther S, Cherry EM, Otani NF, Krinsky V, Pumir A, Bodenschatz E, Gilmour RF. 2009. Termination of atrial fibrillation using pulsed low-energy far-field stimulation. Circulation 120:467-476. DOI: https://doi.org/10. 1161/CIRCULATIONAHA.108.825091, PMID: 19635972

Feola I, Volkers L, Majumder R, Teplenin A, Schalij MJ, Panfilov AV, de Vries AAF, Pijnappels DA. 2017. Localized optogenetic targeting of rotors in atrial cardiomyocyte monolayers. Circulation. Arrhythmia and Electrophysiology 10:e005591. DOI: https://doi.org/10.1161/CIRCEP.117.005591, PMID: 29097406

Gottwald G, Pumir A, Krinsky V. 2001. Spiral wave drift induced by stimulating wave trains. Chaos: An Interdisciplinary Journal of Nonlinear Science 11:487-494. DOI: https://doi.org/10.1063/1.1395624, PMID: 12779486

Kharche SR, Biktasheva IV, Seemann G, Zhang H, Biktashev VN. 2015. A computer simulation study of anatomy induced drift of spiral waves in the human atrium. BioMed Research International 2015:731386. DOI: https:// doi.org/10.1155/2015/731386, PMID: 26587545

Krinski V. 1968. Fibrillation in the excitable media. Problemy Kibernetiki 2:59-80.

Li W, Ripplinger CM, Lou Q, Efimov IR. 2009. Multiple monophasic shocks improve electrotherapy of ventricular tachycardia in a rabbit model of chronic infarction. Heart Rhythm 6:1020-1027. DOI: https://doi.org/10.1016/j. hrthm.2009.03.015, PMID: 19560090

Luther S, Fenton FH, Kornreich BG, Squires A, Bittihn P, Hornung D, Zabel M, Flanders J, Gladuli A, Campoy L, Cherry EM, Luther G, Hasenfuss G, Krinsky VI, Pumir A, Gilmour RF, Bodenschatz E. 2011. Low-energy control of electrical turbulence in the heart. Nature 475:235-239. DOI: https://doi.org/10.1038/nature10216, PMID: 21753855 
Majumder R, Feola I, Teplenin AS, de Vries AA, Panfilov AV, Pijnappels DA. 2018. Optogenetics enables realtime spatiotemporal control over spiral wave dynamics in an excitable cardiac system. eLife 7:e41076. DOI: https://doi.org/10.7554/eLife.41076, PMID: 30260316

Markus M, Nagy-Ungvarai Z, Hess B. 1992. Phototaxis of spiral waves. Science 257:225-227. DOI: https://doi. org/10.1126/science.257.5067.225, PMID: 17794754

Matiukas A, Mitrea BG, Qin M, Pertsov AM, Shvedko AG, Warren MD, Zaitsev AV, Wuskell JP, Wei MD, Watras J, Loew LM. 2007. Near-infrared voltage-sensitive fluorescent dyes optimized for optical mapping in bloodperfused myocardium. Heart Rhythm 4:1441-1451. DOI: https://doi.org/10.1016/j.hrthm.2007.07.012, PMID: 17954405

Newall EG, Lever NA, Prasad S, Hornabrook C, Larsen PD. 2007. Psychological implications of ICD implantation in a New Zealand population. EP Europace 9:20-24. DOI: https://doi.org/10.1093/europace/eul142, PMID: 17224417

Nyns ECA, Kip A, Bart Cl, Plomp JJ, Zeppenfeld K, Schalij MJ, de Vries AAF, Pijnappels DA. 2017. Optogenetic termination of ventricular arrhythmias in the whole heart: towards biological cardiac rhythm management. European Heart Journal 38:2132-2136. DOI: https://doi.org/10.1093/eurheartj/ehw574, PMID: 28011703

Panfilov AV. 2009. Theory of Reentry. In: Panfilov A. V (Ed). Saunders. Elsevier. p. 329-337.

Pertsov AM, Davidenko JM, Salomonsz R, Baxter WT, Jalife J. 1993. Spiral waves of excitation underlie reentrant activity in isolated cardiac muscle. Circulation Research 72:631-650. DOI: https://doi.org/10.1161/01.RES.72.3. 631, PMID: 8431989

Petkova-Kirova PS, London B, Salama G, Rasmusson RL, Bondarenko VE. 2012. Mathematical modeling mechanisms of arrhythmias in transgenic mouse heart overexpressing TNF- $\alpha$. American Journal of PhysiologyHeart and Circulatory Physiology 302:H934-H952. DOI: https://doi.org/10.1152/ajpheart.00493.2011, PMID: 22081697

Pumir A, Sinha S, Sridhar S, Argentina M, Hörning M, Filippi S, Cherubini C, Luther S, Krinsky V. 2010. Wavetrain-induced termination of weakly anchored vortices in excitable media. Physical Review E 81:010901. DOI: https://doi.org/10.1103/PhysRevE.81.010901, PMID: 20365315

Quiñonez Uribe RA, Luther S, Diaz-Maue L, Richter C. 2018. Energy-Reduced arrhythmia termination using global photostimulation in optogenetic murine hearts. Frontiers in Physiology 9:1651. DOl: https://doi.org/10. 3389/fphys.2018.01651, PMID: 30542292

Saito S, Masuda K, Mori Y, Nakatani S, Yoshioka Y, Murase K. 2017. Mapping of left ventricle wall thickness in mice using 11.7-T magnetic resonance imaging. Magnetic Resonance Imaging 36:128-134. DOI: https://doi. org/10.1016/j.mri.2016.10.030, PMID: 27989905

Scardigli M, Müllenbroich C, Margoni E, Cannazzaro S, Crocini C, Ferrantini C, Coppini R, Yan P, Loew LM, Campione M, Bocchi L, Giulietti D, Cerbai E, Poggesi C, Bub G, Pavone FS, Sacconi L. 2018. Real-time optical manipulation of cardiac conduction in intact hearts. The Journal of Physiology 596:3841-3858. DOI: https://doi org/10.1113/JP276283, PMID: 29989169

Wathen MS, DeGroot PJ, Sweeney MO, Stark AJ, Otterness MF, Adkisson WO, Canby RC, Khalighi K, Machado C, Rubenstein DS, Volosin KJ, PainFREE Rx II Investigators. 2004. Prospective randomized multicenter trial of empirical antitachycardia pacing versus shocks for spontaneous rapid ventricular tachycardia in patients with implantable cardioverter-defibrillators: pacing fast ventricular tachycardia reduces shock therapies (PainFREE rx II) trial results. Circulation 110:2591-2596. DOI: https://doi.org/10.1161/01.CIR.0000145610.64014.E4, PMID: 15492306

Wellner M, Zemlin C, Pertsov AM. 2010. Frustrated drift of an anchored scroll-wave filament and the geodesic principle. Physical Review E 82:036122. DOI: https://doi.org/10.1103/PhysRevE.82.036122, PMID: 21230154

Williams JC, Xu J, Lu Z, Klimas A, Chen X, Ambrosi CM, Cohen IS, Entcheva E. 2013. Computational optogenetics: empirically-derived voltage- and light-sensitive channelrhodopsin-2 model. PLOS Computational Biology 9:e1003220. DOI: https://doi.org/10.1371/journal.pcbi.1003220, PMID: 24068903

Woo S-J, Hong JH, Kim TY, Wook Bae B, Lee KJ. 2008. Spiral wave drift and complex-oscillatory spiral waves caused by heterogeneities in two-dimensional in vitro cardiac tissues. New Journal of Physics 10:015005. DOI: https://doi.org/10.1088/1367-2630/10/1/015005

Zipes DP, Fischer J, King RM, Nicoll A , Jolly WW. 1975. Termination of ventricular fibrillation in dogs by depolarizing a critical amount of myocardium. The American Journal of Cardiology 36:37-44. DOI: https://doi. org/10.1016/0002-9149(75)90865-6, PMID: 1146696 
Drift and termination of spiral waves in optogenetically modified cardiac tissue at sub-threshold illumination

\section{Supplementary}



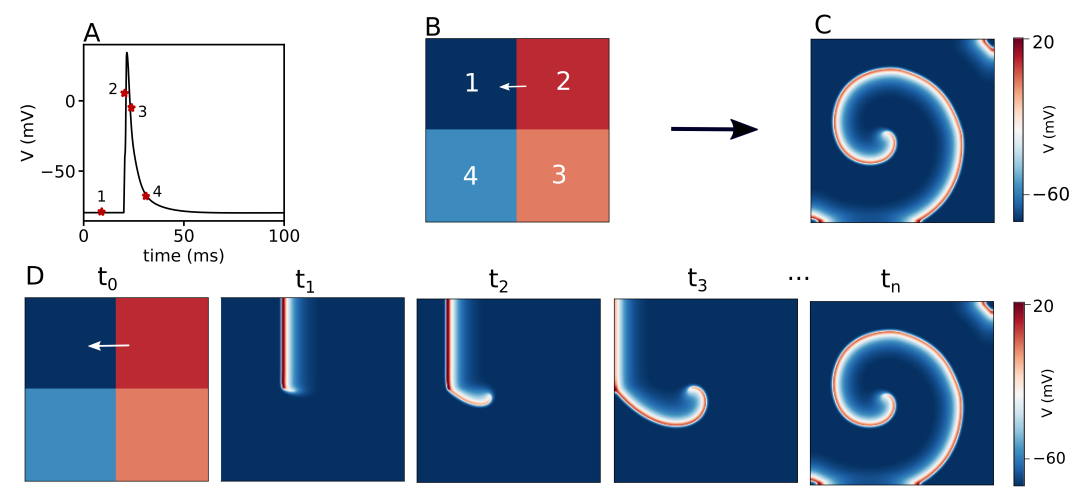

Figure 1: A) four different membrane voltage values are chosen; one value from resting state (1), one value from depolarization state (2), and two values from repolarization state $(3$ and 4$)$. B) the 2 -D mono-domain $(200 \times 200)$ is initialized by four selected values. C) a spiral wave is formed. D) shows a series of frames during formation of the spiral wave

Movie1: Spatial drift of the spiral wave imposed by a LI gradient of $8 \times 10^{4}$ $\mathrm{mW} / \mathrm{mm}^{3}$. The spiral wave drifts along the illumination gradient direction. Finally the spiral wave collides to the boundary and is terminated.

Movie2: Spatial drift of the spiral wave in a domain that is partially illuminated with LI of $0.01 \mathrm{~mW} / \mathrm{mm}^{2}$. Initially the spiral wave drifts fast toward the uniformly-illuminated region, then it slows down due to the homogeneous region far from the interface of the illuminated and non-illuminated regions.

Movie3: Continuous spatial drift of a spiral wave using a multi-step adjusted pattern illumination with LI of $0.01 \mathrm{~mW} / \mathrm{mm}^{2}$. At each step of reducing the size of the illuminated region the illumination was applied with a constant illumination PL of $600 \mathrm{~ms}$. The spiral wave drifts continuously along with the reduction direction of the illuminated region size. Finally it collides to the boundary and is terminated.

Movie4: Drift of a spiral toward the illuminated region with an exponential decay pattern with LI $0.07 \mathrm{~mW} / \mathrm{mm}^{2}$.

Movie5: A spiral wave rotation with no illumination pattern. 

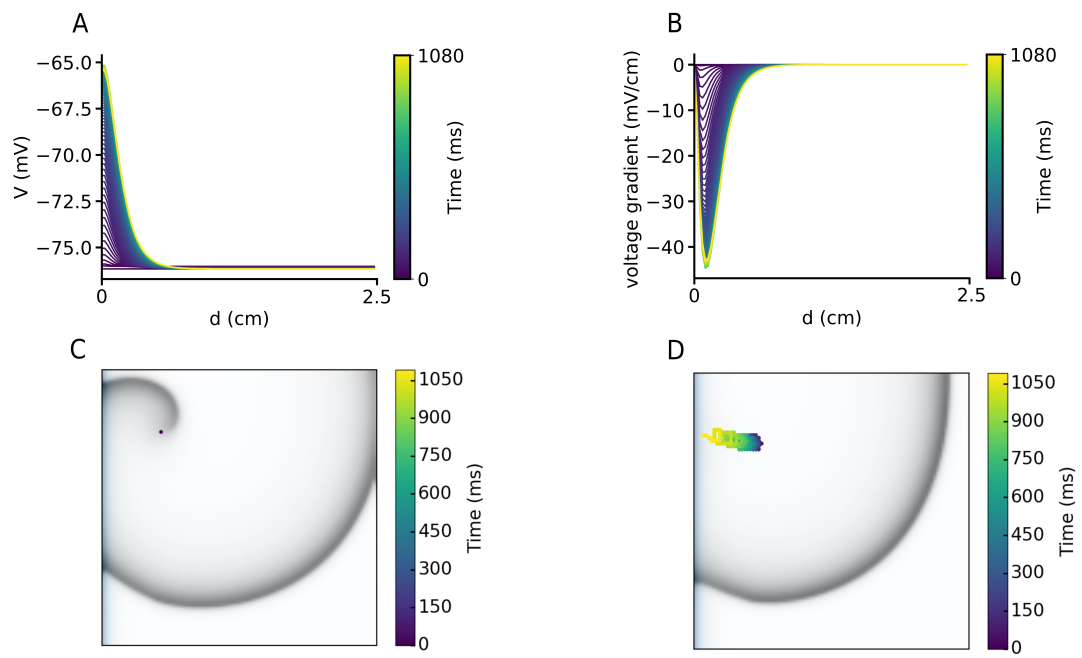

Figure 2: patial drift of a spiral wave imposed by a exponential pattern of subthreshold illumination. A) Time evolution of the voltage distribution along a line perpendicular to the illumination pattern in a quiescent domain. B) Spatial derivative of the voltage distribution $(d V / d x)$ along the line perpendicular to the illumination pattern in a quiescent. C) Initial position of the spiral tip in a domain with an illumination gradient ranging from $\mathrm{LI}=0 \mathrm{~mW} / \mathrm{mm}^{2}$ at the left boundary to $\mathrm{LI}=0.07 \mathrm{~mW} / \mathrm{mm}^{2}$. D) Drift of the spiral wave toward the region with higher LI. 


\section{Chapter 5}

\section{Feedback and non-feedback control of cardiac arrhythmia}

\subsection{Scientific goal and findings}

Some theoretical studies have demonstrated control over the dynamics of spiral waves during arrhythmias by the induction of resonant drift. Such drift is maintained by periodic modulation of the excitability of cardiac tissue, at a frequency close to the dominant frequency of the arrhythmia. In this study, I begin with a numerical induction of resonant drift of a spiral wave with an open loop periodic sub-threshold stimulation, using light of low intensity. I stimulate the spiral wave with light at a fixed frequency. As shown in experiments and computer simulations in chapter 4, illumination decreases the dominant frequency of the arrhythmia. This interferes with the induction of drift, as the stimulation is no longer 'resonant' at the pre-determined frequency of the arrhythmia. To rectify this situation, I apply feedback control to update the frequency of stimulation. To do this, I allow the wave to be stimulated optically only when it passes a measuring point within the domain. This leads to feedback induced resonant drift, where pacing is applied with the most up-to-date frequency of the arrhythmia. To control cardiac arrhythmia in experiments, one possible way to apply feedback pacing is to use the information received from the electrocardiogram (ECG) signal. However, due to the loss of information from this signal during electrical stimulation, it is not possible to control an arrhythmia with electrical pacing using feedback method. However, with optogenetics, it is possible to retain information about the electrical activity of the heart during optical stimulation. This facilitates a controlled, phaselocked stimulation of the spiral wave by using feedback algorithm. In this study, I use optogenetics as a tool to investigate the potential of the feedback pacing method to control cardiac arrhythmia and compare with a non-feedback pacing method. For the experimental study, an electronic feedback device was developed, which could trigger an arrhythmia in a transgenic mouse heart by applying global stimulation at a constant phase based on the feedback algorithm. Optical mapping experiments are still in the progress of demonstrating this phenomenon, feedback-induced resonant drift, in an 
intact transgenic mouse heart.

\title{
Manuscript Status
}

The manuscript is under preparation.

\section{Author Contributions}

- Sayedeh Hussaini designed the research, performed all numerical simulations, performed the experiments, analyzed the experimental and numerical data, and wrote the manuscript

- Aidai Mamyraiym kyzy performed the experiments, analyzed the data, and wrote the manuscript

- Laura Diaz designed the electronic feedback device based on the feedback algorithm

- Johannes Schroeder-Schetelig Optical mapping experiments and analysis

- Raúl A. Quiñonez Uribe Optical mapping experiments and analysis

- Vishalini Venkatesan conducting the experiments

- Claudia Richter the experiments were conducted at her lab

- Vadim Biktashev designed the research and edited the manuscript

- Rupamanjari Majumder designed the research and wrote the manuscript

- Valentin Krinski designed the research and edited the manuscript

- Stefan Luther designed the research and wrote the manuscript

\subsubsection{Manuscript}

\begin{abstract}
Spiral waves in heart tissue rotate with high frequency and override the normal rhythm generated by the pacemaker. Such high frequency electrical activity is accompanied by inefficient contraction of the muscle, which leads to compromised pump function and insufficient cardiac output. Thus, removing spiral waves from the heart is an essential step towards controlling arrhythmias. In this work, we use light-based sub-threshold periodic stimulation to control and study the dynamics of a spiral wave in an ionic model of two dimensional adult mouse ventricular tissue. For this purpose, we use open loop periodic sub-threshold illumination. Depending on the ratio of the stimulation frequency to the frequency of the spiral wave, we obtain epicycloidal, hypocycloidal, and resonant drift trajectories. We observe that illumination alters the frequency of the spiral wave disrupting the process of the resonant drift. To rectify this, we use feedback control to stimulate the spiral wave at a constant phase instead of a constant frequency. Using this method we can direct the wave to drift in a desired direction.
\end{abstract}


Finally, we provide some ex vivo studies on an optogenetically modified intact mouse heart to demonstrate the accuracy and potential benefits of this method.

\section{Introduction}

Anomalies in the generation and conduction of electrical signals in the heart lead to instabilities, which often result in the formation and sustenance of spiral waves in two dimensional (2D), and scroll waves in three dimensional (3D) cardiac tissue. These rotating waves are associated with life-threatening cardiac rhythm disorders, such as ventricular fibrillation [191-193]. The state-of-the-art technique for suppressing fibrillation in the clinic involves applying a single, high-voltage electric shock to the heart to reset all electrical activity, allowing the natural pacemaker to restore a regular rhythm $[194,195]$. Despite its high success rate, this defibrillation technique comes with a wide range of harmful side-effects such as, intense pain, trauma, anxiety and depression $[4,5]$. Therefore alternative less harmful methods with comparable efficiency are being intensively researched [6-9]. Further progress in the clinical implementation of these developing techniques requires a deeper understanding of the underlying spiral and scroll wave dynamics. A particularly interesting dynamical feature observed in the presence of structural and functional inhomogeneities in the heart is spiral wave drift [196-199]. This feature forms the basis for termination of spiral wave activity in the heart by the method of anti-tachycardia pacing (ATP) [63-65]. The ATP method uses a train of high- frequency, low-energy electric pulses to induce drift of spiral wave cores towards an inexcitable boundary, where they annihilate upon collision [66-68]. Application of a spatially uniform low-amplitude electrical perturbation to the spiral wave with a frequency close to its natural rotation frequency also causes spiral wave drift through resonance [200]. Such drift is referred to as resonant drift of the spiral wave. Resonant drift first was predicted analytically by Davydov et al. [201] and shown in experiments by Agladze et al. in a Belousov-Zhabotinsky (BZ) reaction system [92]. Presence of inhomogeneities in real cardiac tissue, the influence of external parameters, and boundaries cause the direction of the resonant drift to deviate from linearity [199, 200]. However, such deviations can be overcome in a robust manner by applying dynamic feedback [189, 202-204]. The approach relies on continuously updating the frequency of the applied perturbation, based on the variation of the rotation frequency of the spiral wave. Due to the loss of information of the electrical activity in the electrocardiogram (ECG) signal during electrical perturbations, conventional methods can not be used to induce feedback induced resonant drift. However, indirect methods, such as optogenetics, which is based on using light to trigger the production of current by cardiac cells to depolarize or hyperpolarize the cell membrane, maintain the electrical activity information in the ECG signal during optical stimulation. Therefore optogenetics can be used as a valuable tool for proof-of-concept for feedback induced resonant drift. Recent numerical and experimental studies using optogenetics have demonstrated the enormous potential of this technology to be used as a tool for investigating and controlling spiral wave dynamics at supra-threshold, where an action 
Feedback and non-feedback control of cardiac arrhythmia

potential occurs and is followed by excitation wave propagation [11, 23, 99, 205, 206], as well as sub-threshold [207] light intensities, where no action potential is triggered.

In this study, we demonstrate the induction of resonant drift of a spiral wave in simulated 2D light-responsive cardiac tissue, using sub-threshold illumination pacing with a fixed frequency and adjustable frequency using feedback method. Application of light perturbations to cardiac tissue, results in a time-dependent change in the membrane voltage, which in turn produces a nonlinear shift in the natural frequency of the spiral wave. Thus, by using dynamical feedback to perturb the spiral wave in a controlled phase-locked manner, we demonstrate rectified resonant drift. In the scope of this method, using a measuring electrode in the domain, we stimulate the spiral wave when it touches this electrode. The direction of the drift is a function of the electrode position. Hence, with this method we can guide the wave in a desired direction. To show the applicability of such a robust method based on a thirty years old theory, feedback induced resonant drift, we provide some experimental data showing termination of arrhythmia in the intact transgenic mouse heart using global illumination.

\section{Methods and Materials}

\section{Numerical Study}

For the 2D computational studies in light-responsive cardiac tissue, we considered a simulation domain containing $100 \times 100$ grid points, each grid point represents a cardiac ventricular cell from an adult mouse. Electrical activity in this domain was described using a reaction-diffusion-type partial differential equation as following:

$$
\frac{d V}{d t}=\nabla \cdot D \nabla V-\frac{I_{i o n}+I_{C h R 2}}{C_{m}}
$$

Where $\mathrm{V}$ is the membrane voltage of a single cardiac cell, $\mathrm{C}_{m}$ is the membrane capacitance, $D$ is the diffusion coefficient with a value of $0.00014 \mathrm{~cm} / \mathrm{ms}$ to produce a conduction velocity $(\mathrm{CV})$ of $43.9 \mathrm{~cm} / \mathrm{s}$, and $\mathrm{I}_{\text {ion }}$ is the net ionic current flowing across the cell membrane. We describe $\mathrm{I}_{i o n}$ according to the Bondarenko model for adult mouse ventricular cells $[34,35]$. In order to incorporate light-sensitivity to the cardiac cells we coupled a 4-state model of an light-responsive protein Channelrhodopsin-2 (ChR2) [36] to this cell model. This model is described mathematically as follows:

$$
I_{C h R 2}=g_{C h R 2} G(V)\left(O_{1}+\gamma O_{2}\right)\left(V-E_{C h R 2}\right)
$$

Where $\mathrm{g}_{C h R 2}$ is the conductance, $\mathrm{G}(\mathrm{V})$ is the voltage rectification function, $\mathrm{O}_{1}$ and $\mathrm{O}_{2}$ are the open state probabilities of the ChR2, $\gamma$ is the ratio $\mathrm{O}_{1} / \mathrm{O}_{2}$, and $\mathrm{E}_{C h R 2}$ is the reversal potential of this channel. 25 different initial conditions were considered for all numerical studies. 


\section{Experimental Study}

To study dose-response curve of termination rate of arrhythmia at different light intensities (LI) and pulse length (PL) of $20 \mathrm{~ms}$, we applied a single global illumination to the heart of $\alpha$-MHC-ChR2 transgenic mice in Langendorff vertically perfused with normal Tyrode. This solution preserves the electrophysiological conditions of the heart and contains $30 \mathrm{mM} \mathrm{NaCl}, 4 \mathrm{mM} \mathrm{KCl}, 1 \mathrm{mM} \mathrm{MgCl}_{2}, 24 \mathrm{mM} \mathrm{NaHCO}_{3}, 1.8 \mathrm{mM} \mathrm{CaCl}_{2}$ , $1.2 \mathrm{mM} \mathrm{KH}_{2} \mathrm{PO}_{4}, 5.6 \mathrm{mM}$ glucose, $1 \%$ albumin/BSA were aerated with Carbogen (95\% oxygen and $5 \% \mathrm{CO}_{2}$ ) [186]. To induce a persistent ventricular arrhythmia, we used a low potassium normal Tyrode, the $\mathrm{KCl}$ concentration was reduced from $4 \mathrm{mM}$ (normal styrene) to $2 \mathrm{mM}$, with a combination of $100 \mu \mathrm{M}$ Pinacidil to shorten APD [187, 188]. After 20 minutes of perfusion, an arrhythmia was induced by applying 30 electrical pulses with the amplitude of $2.3-2.5 \mathrm{~V}$ and the frequency of $30-50 \mathrm{~Hz}$ using a needle electrode. To provide a global illumination a single blue light with a certain PL was applied using three LEDs located at three different sides around the bath with angular separation of $120^{\circ}$. To stimulate an intact mouse heart in a controlled phase-locked manner using the feedback method, we have developed an electronic device which triggers an arrhythmia at a constant phase. We show termination of arrhythmia in intact mouse heart using feedback pacing. All the experiments were conducted on 5 mouse hearts with the number of 10 trials for each.

\section{Results}

Lethal cardiac arrhythmias, such as ventricular tachycardia and fibrillation, are associated with the organization of spiral waves in the heart. These high-frequency waves override sinus rhythm to drive the cardiac electromechanical pump with reduced efficiency. Elimination of these waves from the heart is essential for the termination of the arrhythmia. However, effective elimination of spiral waves requires a deep understanding of spiral wave dynamics in cardiac tissue. Theoretical studies by Davydov et al. [201] demonstrate an approach to control spiral waves by periodic stimulation of the tissue, below its excitation threshold. Here, we demonstrate a similar method for spiral wave control in cardiac tissue using optogenetics in the sub-threshold illumination régime.

To begin with, we induce a spiral wave in a two dimensional (2D) simulation domain, containing optogenetically-modified mouse ventricular cardiomyocytes. The spiral rotates with a frequency $f_{s}=15.62 \mathrm{~Hz}$ around a circular core (see Fig.5.1-A). Next, we apply periodic global sub-threshold illumination (light intensity LI $=20$ $\mu \mathrm{W} / \mathrm{mm}^{2}$ and pulse width $\mathrm{PL}=32 \mathrm{~ms}$ ) to this domain at a fixed frequency $f_{p}$. At different values of $f_{p}$, the spiral wave exhibits different dynamical behaviour. These are illustrated in Fig 5.1-B, C, and D, which show, respectively, trajectories of the spiral wave tip at $f_{p}=15.62,13.33$, and $12.5 \mathrm{~Hz}$. In each case, the spiral wave either meanders with a hypocyclidal or epicycloidal trajectory or drifts along a cycloidal path. 


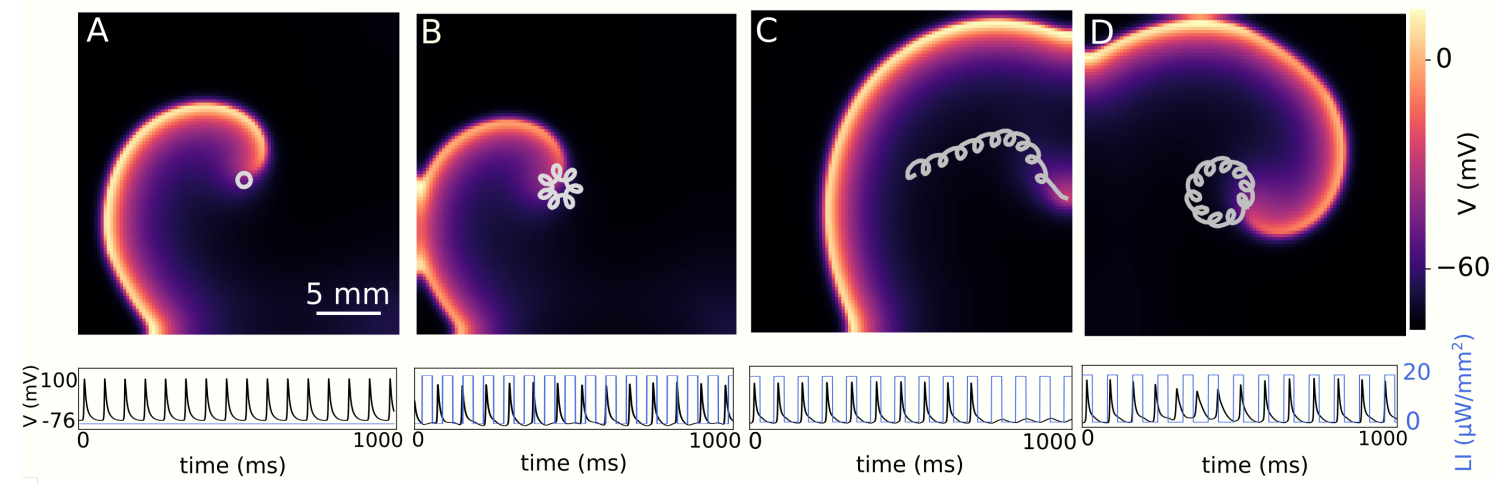

Figure 5.1 - Different meandering patterns of a spiral wave's tip trajectory A) Circular trajectory of a spiral wave's tip with no-illumination in a homogeneous $2 D$ domain. $B$ ) Hypocycloidal trajectory of the spiral wave's tip via a global periodic illumination with the $f_{p}$ equals to initial $f_{s}$ with value of $15.6 \mathrm{~Hz}$ and $\mathrm{LI}$ of $20 \mu \mathrm{W} / \mathrm{mm}^{2}$. C) Trajectory of the spiral wave's tip via global periodic illumination with the $f_{p}$ of $13.33 \mathrm{~Hz} . \mathrm{D}$ ) Epicycloidal trajectory of the spiral wave's tip via a global periodic illumination with the $f_{p}$ of $12.5 \mathrm{~Hz}$ and $\mathrm{LI}$ of $20 \mu \mathrm{W} / \mathrm{mm}^{2}$.

Application of an external force to a rotating spiral wave leads to a resonant drift when $f_{p}=f_{s}$, whereby the spiral wave is triggered at constant phase [200]. However, we observed a hypocycloidal pattern of trajectory when $f_{p}$ was applied equal to the initial $f_{s}(15.6 \mathrm{~Hz})$, as shown in Fig.5.1-B. In a previous study, I showed using experiments and simulation that sub-threshold illumination decreases the conduction velocity of a propagating signal, as well as the frequency of a spiral, i.e., $f_{s}$. The present findings are in line with the previous observations in that we observe a drop in the frequency of the spiral wave in the presence of sub-threshold illumination. Fig.5.2-A shows the voltage time series $V(0.75 \mathrm{~cm}, 1.75 \mathrm{~cm}, t)$ recorded from a representative point $(0.75$ $\mathrm{cm}, 1.75 \mathrm{~cm})$ of the simulation domain for $t=1 \mathrm{~s}$ and periodic pacing with $f_{p}=15.62$ Hz. Clearly, application of the light stimulus reduces $f_{s}$ from $15.62 \mathrm{~Hz}$ to almost 13.3 Hz. Thus, $f_{p}=15.62 \mathrm{~Hz}$ is actually treated by the spiral as overdrive pacing, with the trigger occurring in a non-consistent phase of the spiral wave, (see Fig.5.2- $\mathrm{A}^{\prime}$ ). This results in a meandering motion of the spiral tip with a hypocyloidal pattern (see Fig.5.1-B). In order to induce resonant drift, it is essential to match the frequency of the applied pacing with the updated frequency of the spiral wave. Thus, we decrease $f_{p}$ to a value close to the new $f_{s}$. Voltage time-series in Fig.5.2-B and B' show that such reduction of $f_{p}$ also ensures consistent triggering of the spiral wave by the pacing stimulus in a fixed phase. Here $f_{p} \simeq f_{s}=13.33 \mathrm{~Hz}$. This results in a resonant drift as it is shown in Fig.5.1-C. Further decrease of the $f_{p}$ leads to under-drive pacing where $f_{p}$ is less than $f_{s}$. The corresponding voltage time series recorded from a representative point of the domain during $1 \mathrm{~s}$ of the pacing is shown in Fig.5.2-C. Fig.5.2- $\mathrm{C}^{\prime}$ gives a closer look to the time series corresponding to the Fig.5.2-C. The corresponding tip trajectory of the spiral wave shows a characteristic epicycloidal pattern (see Fig.5.1-B, where $f_{p}<f_{s}$ ). 


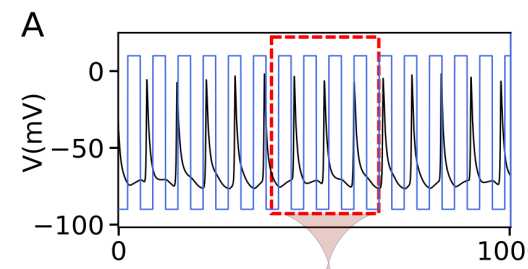

$A^{\prime}$

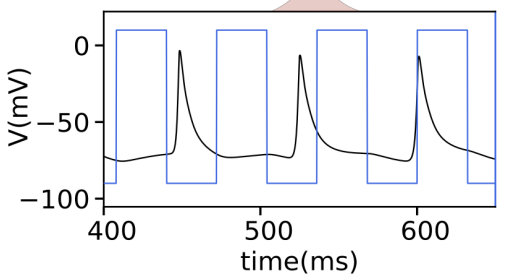

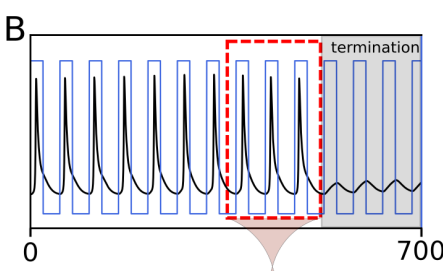

$B^{\prime}$

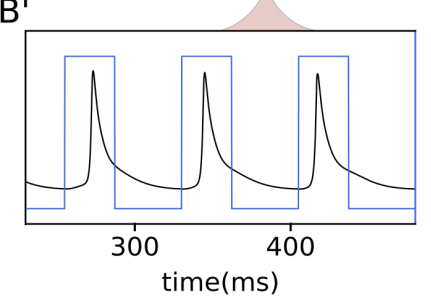

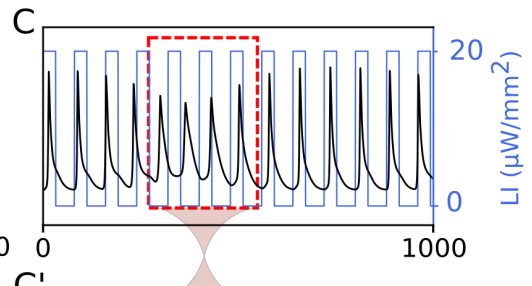

$\mathrm{C}^{\prime}$

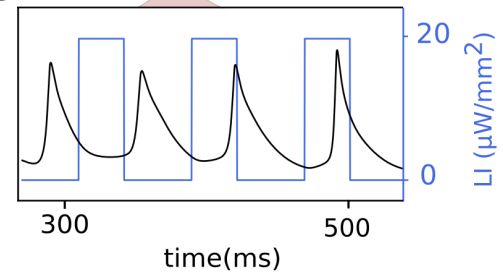

Figure 5.2 - Voltage time series of a single point at the domain with different pacing frequencies. A) A voltage time series of a single point in the domain when a periodic illumination is applied with the $f_{p}$ of $15.62 \mathrm{~Hz}$ and the $L I$ of $20 \mu \mathrm{W} / \mathrm{mm}^{2}$. The number of action potential and pacing pulses demonstrates $\left.f_{p}>f_{s} . A^{\prime}\right)$ A closer look at the voltage series and the illumination pulses shown in panel $(A)$. We see that spiral wave is not triggered at a constant phase. B) A voltage time series of a single point in the domain when there is a periodic illumination with $f_{p}$ of $13.33 \mathrm{~Hz}$ and the $L I$ of $20 \mu \mathrm{W} / \mathrm{mm}^{2}$. The number of action potential and pacing pulses demonstrates $f_{p}=f_{s}$. This results in the termination of the spiral wave, shown with a gray shaded area. $\left.B^{\prime}\right) A$ closer look at the voltage series and the illumination pulses is shown in panel (B). It shows that the spiral wave is triggered at an almost constant phase. C) A voltage time series of single point in the domain when a periodic illumination is applied with $f_{p}$ of $12.5 \mathrm{~Hz}$ and LI of $20 \mu \mathrm{W} / \mathrm{mm}^{2}$. The number of action potential and pacing pulses demonstrates $f_{p}>f_{s}$. $\left.C^{\prime}\right)$ A closer look at the voltage series and the illumination pulses is shown in panel $(C)$. It shows that the spiral wave is not triggered at a constant phase.

As shown above, control and termination of the spiral wave using open loop periodic pacing to induce resonant drift is limited to a certain range of $f_{p}$ depending on the applied light intensity (LI). Due to the decrease of $f_{s}$ upon illumination, this range of $f_{p}$ is lower than the $f_{s}$ without illumination. A frequency slightly higher or lower than this range leads to overdrive or under-drive pacing which results in meandering patterns of the tip's trajectory. To increase our control over the dynamics of the spiral wave, in order to steer it in a desired direction, we used a method known as 'feedback loop'. In this method, the spiral wave is triggered when the front-wave touches a measuring electrode located in the domain. This results in pacing the spiral wave with a frequency adjusted to the most recent $f_{s}$. Fig.5.3 shows the trajectory of the spiral wave tip with feedback pacing when the electrode is positioned at different regions of the domain. In Fig.5.3, the filled red circle indicates the position of the measuring electrode. In all cases, an optical stimulus is delivered without delay, every time the wavefront passes the measuring electrode and the membrane voltage is at a critical value $V_{c}$. Here, we chose $V_{c}=-40 \mathrm{mV}$. Below each figure shown in Fig.5.3 A-C, a voltage time series, recorded at the position of the measuring electrode, is 
Feedback and non-feedback control of cardiac arrhythmia

provided. Due to the change of $f_{s}$ with respect to the measuring electrode, the Doppler effect, the spiral wave exhibits a drift-type motion that circularly envelops the location of the measuring electrode (see Fig.5.3 A-C). Exploiting the notion that the spiral wave circulates around the measuring electrode, we could drive the former toward an inexcitable boundary by placing the measuring electrode in one of the corners of the simulatiuon domain. Fig.5.3-D shows the collision of the spiral wave with the boundary when the measuring electrode is in the lower right corner of the domain.
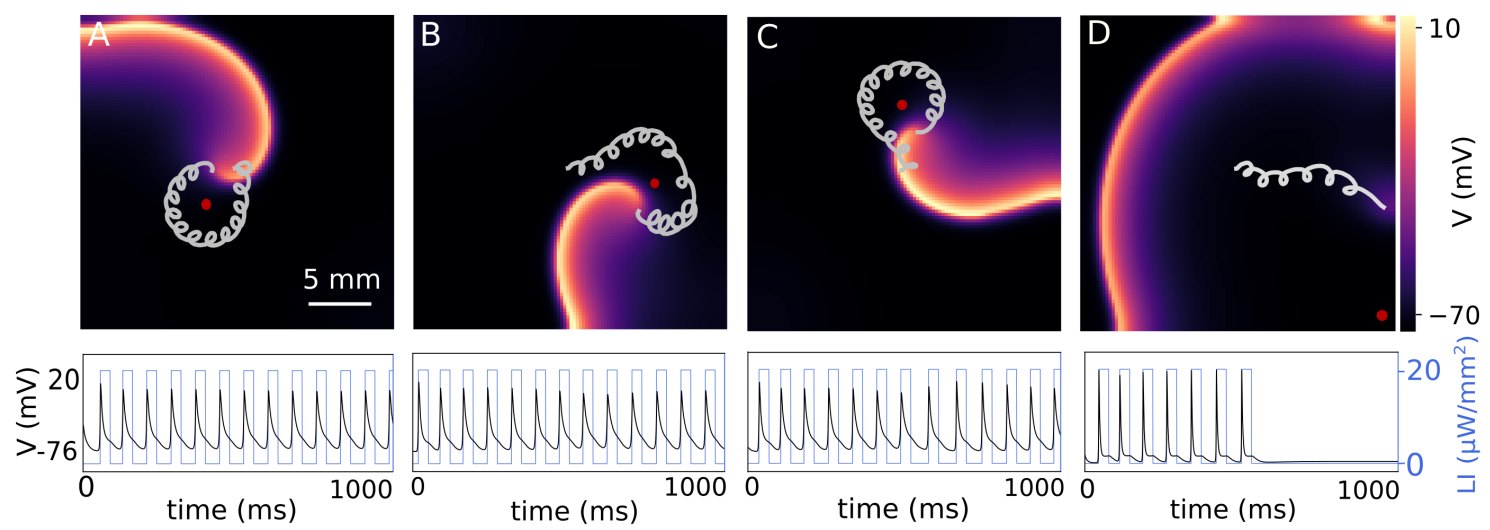

Figure 5.3 - Driving the spiral wave using feedback control. A) Circular trajectory of the spiral wave where the measuring electrode is located at the coordinate of $(1 \mathrm{~cm}, 1$ $\mathrm{cm})$. Corresponding voltage series of the measuring electrode shows triggering the spiral wave at a constant phase with the critical voltage value of $-40 \mathrm{mV}$. B) Circular trajectory of the spiral wave where the measuring electrode is located at the coordinate of (1.875 $\mathrm{cm}, 1.25 \mathrm{~cm}$ ). Corresponding voltage series of the measuring electrode shows triggering the spiral wave at a constant phase with the critical voltage value of $-40 \mathrm{mV}$. C) Circular trajectory of the spiral wave where the measuring electrode located at the coordinate of $(1.25 \mathrm{~cm}, 1.875 \mathrm{~cm})$. Corresponding voltage series of the measuring electrode shows triggering the spiral wave at a constant phase with the critical voltage value of $-40 \mathrm{mV}$. D) Linear drift of the spiral wave and collision with the boundary when the electrode is located at the lower-right corner of the domain. Corresponding voltage series of the measuring electrode shows triggering the spiral wave at a constant phase with the critical voltage value of $-40 \mathrm{mV}$. Termination of the spiral wave occurs approximately during half of the simulation time.

The mechanism of resonant drift, hypocycloid, epicycloid and feedback drift trajectories can be represented by a simple geometric view. Periodic illumination below the threshold changes the excitability of cardiac tissue, which affects the curvature of the spiral wave's tip trajectory. Fig.5.4-A shows a scheme of the tip trajectory of the spiral wave during resonant drift. The application of periodic illumination at a frequency close to the spiral wave frequency $\left(f_{p}=f_{s}\right)$ causes, during the first half period of the spiral wave rotation $(\pi)$, when the light is on, the trajectory of the tip has a greater curvature (shown with a blue curve) compared to the second half period of the spiral wave, when the light is off, the curvature of the tip trajectory is smaller (shown with 
a gray curve). This leads to a linear drift of the spiral wave. Fig.5.4-A' shows the trajectory of the tip of a spiral wave during periodic sub-threshold illumination when $f_{p}=f_{s}$ with the value of $13.33 \mathrm{~Hz}$. If $f_{p}$ does not coincide with $f_{s}$, this leads to a cycloidal movement of the tip of the spiral wave. Fig.5.4-C shows a scheme of the tip's trajectory, where $f_{p}>f_{s}$. This results in a complete half rotation $(\pi)$ of the tip of the spiral wave when light is on and a rotation of less than half the period $(\pi-\theta)$ when light is off. This leads to a hypocyloidal trajectory with pedals pointing outward. Fig.5.4- $\mathrm{C}^{\prime}$ shows the trajectory of the tip of a spiral wave of a simulation study during periodic illumination when $f_{p}>f_{s}$, where $f_{p}=15.62 \mathrm{~Hz}$ and $f_{s}=13.3 \mathrm{~Hz}$. Fig.5.4-D shows a scheme of the tip trajectory, where $f_{p}<f_{s}$. This results in a complete half rotation $(\pi)$ of the tip of the spiral wave when light is on and a rotation of more than half the period $(\pi+\theta)$ when light is off. This leads to a epicycloidal trajectory with pedals pointing inward.

A

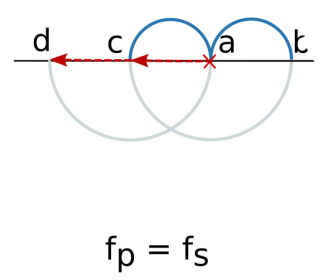

$A^{\prime}$

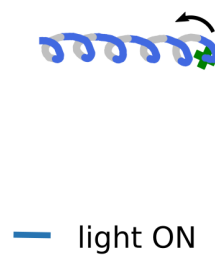

B

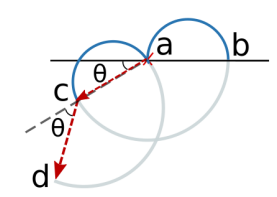

$f_{p}>f_{s}$

$B^{\prime}$

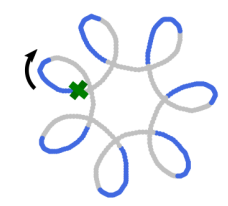

- light OFF
C

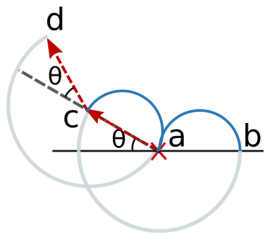

$f_{p}<f_{s}$

$C^{\prime}$

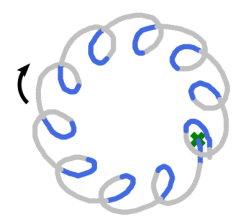

$\times \times$ starting point
D

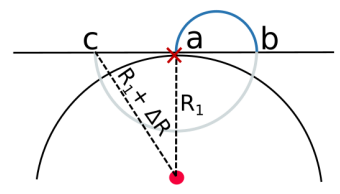

feedback pacing

$D^{\prime}$

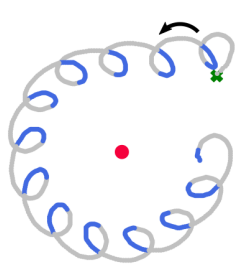

- measuring electrode

Figure 5.4 - Underlying mechanism of the resonant drift, hypocycloidal trajectory, epicycloidal trajectory, and feedback drift. A) Schematic representation of a spiral wave's tip during two rotations where $f_{p}=f_{s}$. $A^{\prime}$ ) Trajectory of the spiral wave in a simulation study where $f_{p}=f_{s}=13.33 \mathrm{~Hz}$. B) Schematic representation of hypocycloidal trajectory of a spiral wave's tip during first two rotations where $f_{p}>f_{s} . B^{\prime}$ ) Hypocyloidal trajectory of a spiral wave of a simulation study where $f_{p}=15.62 \mathrm{~Hz}$ and $f_{s}=13.33 \mathrm{~Hz} . \mathrm{C}$ ) Schematic representation of epicyloidal trajectory of a spiral wave's tip during first two rotations where $f_{p}<f_{s}$. $\left.C^{\prime}\right)$ Epicyloidal trajectory of a spiral wave in a simulation study where $f_{p}=12.5 \mathrm{~Hz}$ and $f_{s}=13.3 \mathrm{~Hz}$. D) Schematic of feedback drift during one rotation. Increase of distance between the measuring electrode (indicate by a red circle marker) and the tip of the spiral wave from $R_{1}$ to $R_{1}+\Delta R$ causes under-drive pacing. $D^{\prime}$ ) Trajectory of the spiral wave in a simulation study where the measuring electrode is located in the middle. 
Feedback and non-feedback control of cardiac arrhythmia

Fig.5.4-D' shows the trajectory of the tip of a spiral wave of a simulation study during periodic illumination when $f_{p}>f_{s}$, where $f_{p}=12.5 \mathrm{~Hz}$ and $f_{s}=13.3 \mathrm{~Hz}$. Fig.5.4-G shows a single rotation of the spiral wave during feedback control stimulation. The initial position of the tip, (shown with red cross marker at the position of "a") with respect to the measuring electrode (shown in a red circle marker) increases from $R_{1}$ to $R_{1}+\Delta R$, where the tip is at the end of one rotation (at the position of "c"). Therefore, there is a delay time at each rotation with respect to the measuring electrode during feedback control stimulation. This results in an underdrive pacing with an epicycloid meander pattern of the tip trajectory that forms around the measuring electrode. Fig.5.4-G' shows the trajectory of a spiral wave of a simulation study during stimulation with feedback control method.

Based on $f_{p}$ and $f_{s}$ the angle between the initial position of the tip (shown with "a" in Fig.5.4 A-D) and final position during the first rotation (shown with "c" in Fig.5.4 A-D) is as following:

$$
\theta= \pm 2 \pi\left(1-\frac{f_{s}}{f_{p}}\right)
$$

Here, the positive sign is for the case of the tip trajectory with hypocyloidal pattern and negative sign is for the case of tip trajectory with epicycloidal pattern. This equation (5.3) shows during resonant pacing, $f_{s}=f_{p}, \theta$ is equal to zero. Using equation (5.3) the number of pedals $(\mathrm{N})$ required for a complete circle of epicycloidal or hypocycloidal trajectory can be calculated as following:

$$
N=\frac{2 \pi}{\theta}
$$

Control of spiral wave dynamics has been extensively investigated in numerical and analytical studies. Only some of these findings have been validated in experiments and clinical research. Our numerical results prove the potential for optogenetics to be used as a tool for the experimental study of the phonamenon of resonant drift. The ability of the system to retain information about the electrical signal, that is received from the ECG during optical stimulation, makes optogenetics a suitable candidate for the experimental study of resonant drift. To demonstrate that resonant drift can be used to control cardiac arrhythmias in experiments, we apply global illumination at very low light intensities to a transgenic heart of mice during the arrhythmic state of the heart. We induce resonant stimulation using an electronic feedback device that performs the feedback algorithm. Fig.5.5 shows two voltage time series of the monophasic action potential (MAP) during a cardiac arrhythmia of the mouse heart, which are triggered resonantly with a sequence of optical pulses with the $V_{c}$ of $0 \mathrm{mV}$.

To control arrhythmia in the intact mouse heart, we use two different methods: Feedback pacing and non-feedback pacing. For this purpose, we apply global illumination at different LI in the range of $(3.1,103) \mu \mathrm{W} / \mathrm{mm}^{2}$ and PL of $20 \mathrm{~ms}$, which is almost half of the arrhythmia period in the mouse heart. To determine the termination rate of feedback pacing, we apply $10 \mathrm{~s}$ of optical stimulation. If the arrhythmia is 

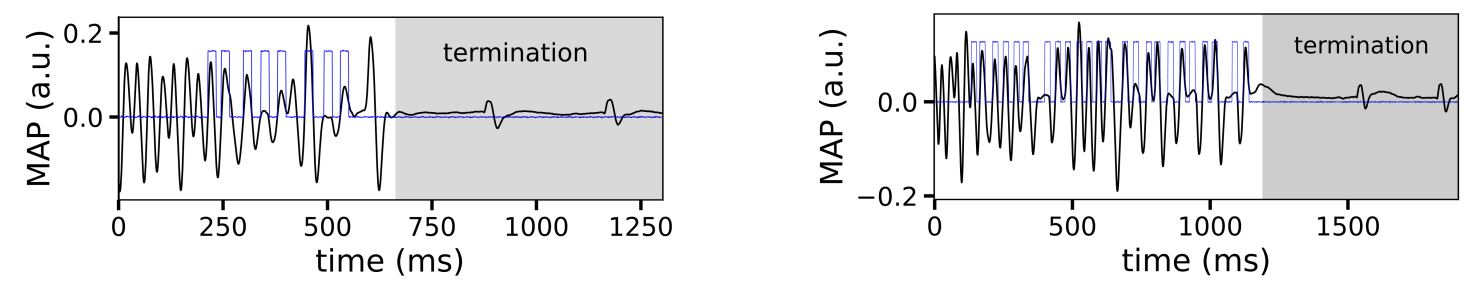

Figure 5.5 - Voltage time series of cardiac arrhythmias triggered at $V_{c}$ of $0 \mathrm{mV}$ using feedback stimulation.

terminated within this period, we call it a success case and if not, a failure case. For the non-feedback stimulation case, we apply a sequence of global optical pulses with a frequency of $30 \mathrm{~Hz}$ and $\mathrm{PL}=20 \mathrm{~ms}$. During the feedback experiments, the average number of pulses for the range of $\mathrm{LI}>11.2 \mu \mathrm{W} / \mathrm{mm}^{2}$ to terminate the arrhythmia is 30 cycles. Therefore, during non-feedback pacing, we apply 30 cycles of optical pulses for the range of LI $>11.2 \mu \mathrm{W} / \mathrm{mm}^{2}$ and $10 \mathrm{~s}$ of optical pacing for the range of LI $\leqslant 11.2 \mu \mathrm{W} / \mathrm{mm}^{2}$. Fig.5.6-A shows the termination rate using feedback pacing and overdrive pacing. It shows higher arrhythmia termination efficiency using feedback pacing compared to overdrive pacing. The green dashed line shows the border of the excitation threshold determined by a sequence of optical pulses with PL of $20 \mathrm{~ms}$ and frequency of $30 \mathrm{~Hz}$ during sinus rhythm.

To have a comparison with the numerical simulations, we apply 10 periodic global optical pulses with different LIs to control the dynamics of the spiral wave in a $2 \mathrm{D}$ domain. The range of the applied LI is $(5,100) \mu \mathrm{W} / \mathrm{mm}^{2}$ and the illumination PL is $33 \mathrm{~ms}$, half the period of the spiral wave. To terminate the spiral wave, we first applied feedback pacing with a measurement electrode in one corner of the area with coordinates (2.475 mm, $0.25 \mathrm{~mm}$ ), shown in Fig.5.3-D. During feedback stimulation, the spiral wave is resonantly stimulated when the wave front passes the measuring electrode and the membrane voltage exceeds the critical value $\mathrm{V}_{c}=-40 \mathrm{mV}$. To investigate the termination rate of the non-feedback pacing, the system is overdriven by 10 optical pulses with $\mathrm{PL}=33 \mathrm{~ms}$ and $f_{p}=16.66 \mathrm{~Hz}$, where the $f_{s}=15.6 \mathrm{~Hz}$. Fig.5.6-B shows the termination rate of the spiral wave against different LIs for feedback pacing and overdrive pacing. The green dashed line shows the excitation threshold, where LI $\leqslant$ $40 \mu \mathrm{W} / \mathrm{mm}^{2}$ corresponds to the sub-threshold and LI $>40 \mu \mathrm{W} / \mathrm{mm}^{2}$ corresponds to the supra-threshold stimulation. These in silico studies show, in agreement with ex vivo, the higher termination efficiency of arrhythmia by feedback pacing compared to supra-threshold pacing. A possible explanation for this observation is that the spiral wave meanders in a certain region of the domain during overdrive pacing without being directed towards the inexcitable boundary, as shown in Fig.5.1-B.

Fig.5.6-C shows a space-time diagram of the membrane voltage along the $\mathrm{x}$-axis of the domain at $\mathrm{y}=12.5 \mathrm{~mm}$ during feedback pacing at different LIs. The white dashed line shows the direction of the drift. It shows an increase in the rotation period of the spiral wave $\left(\mathrm{T}_{s}\right)$ and its conduction velocity $(\mathrm{CV})$ as the LI is increased during 
Feedback and non-feedback control of cardiac arrhythmia

sub-threshold stimulation. Moreover, as the LI is increased from 15 to $40 \mu \mathrm{W} / \mathrm{mm}^{2}$, the drift velocity increases and the spiral wave is terminated again in a shorter time, the mechanism has transitioned to abrupt termination at LI $\geqslant 50 \mu \mathrm{W} / \mathrm{mm}^{2}$. Thus, there is a transition of the termination mechanism from low to high LIs.
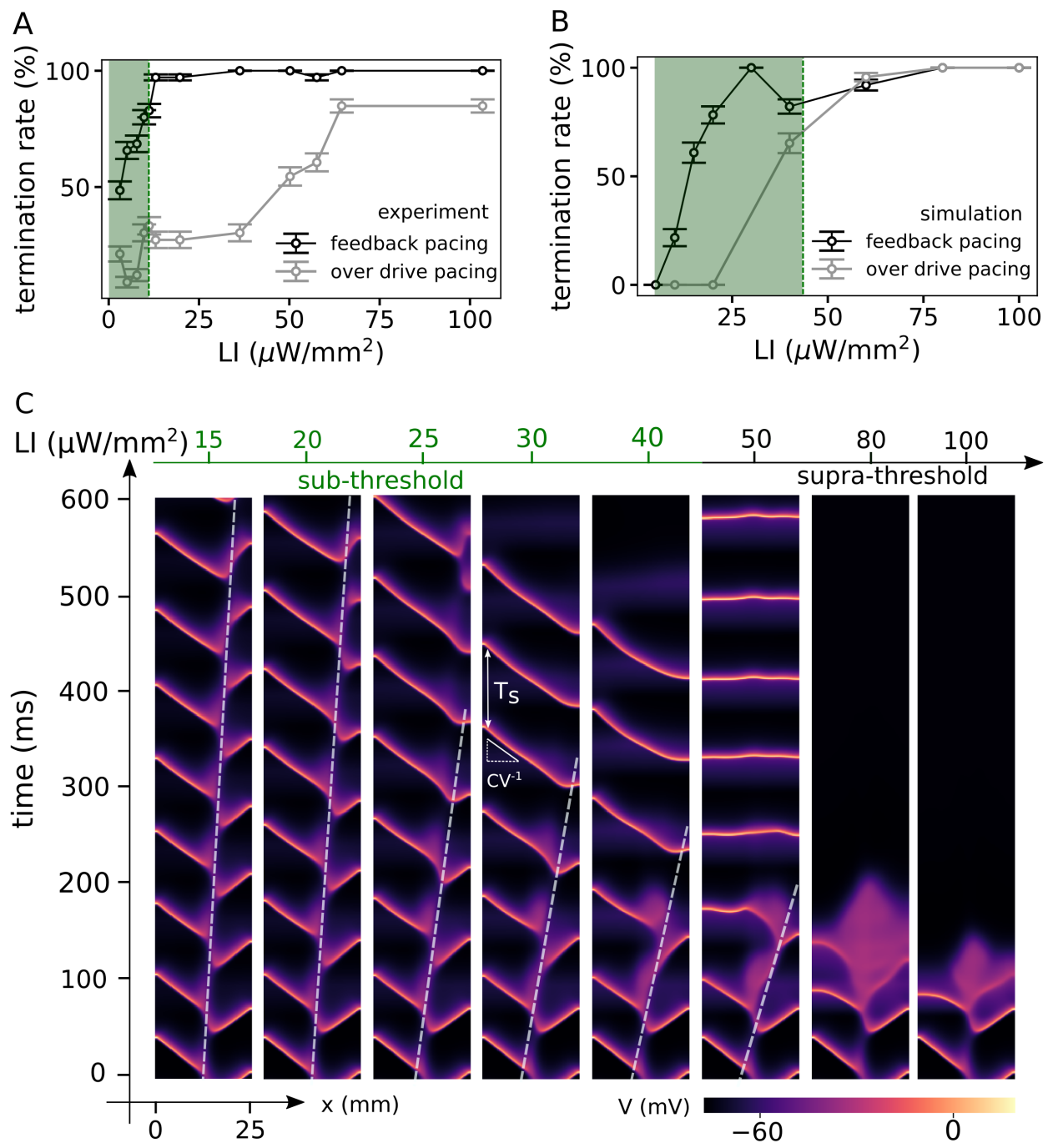

Figure 5.6 - Comparison of resonant feedback and overdrive pacing in silico and ex vivo. A) Arrhythmia termination rate, ex vivo mouse heart $(N=5)$, using resonant feedback pacing and overdrive pacing (percentage of termination rate reported as mean $\pm S E M$ ). It shows a higher efficiency of arrhythmia termination when resonant feedback pacing is used compared to overdrive pacing. B) Termination rate in silico $(N=25)$ using resonant feedback pacing (black) and overdrive pacing (gray) (percentage of termination rate reported as mean $\pm S E M$ ). Resonant feedback pacing shows a higher efficiency of arrhythmia termination using feedback pacing compared to overdrive pacing, which is consistent with the in silico study. C) Space-time plot of the membrane voltage along the $\mathrm{x}$-axis at $\mathrm{y}=12.5 \mathrm{~mm}$, during resonant feedback pacing at different LIs. Increase of LI results in the increase of the spiral wave period $\left(T_{s}\right)$ and its conduction velocity $(C V)$. 
Numerically, we show that the underlying mechanism of termination at sub-threshold is resonant drift, where the spiral wave is gradually pushed towards the inexcitable boundary by sub-threshold feedback pacing. In contrast, with supra-threshold stimulation, the entire domain is excited at once, resulting in abrupt termination.

We showed a higher termination efficiency in both in silico and ex vivo using feedback pacing in comparison with non-feedback pacing. We further studied the number of pulses to terminate the arrhythmia as well as the termination time in the case of feedback pacing. Fig.5.7-A and $\mathrm{A}^{\prime}$ show the the number of pulses to terminate arrhythmia for various LI in silico and ex vivo, respectively. The median values (red line) and mean values (green almond marker) show a decreases in number of pulses for increasing LI. Higher number of pulses at low LIs might be due to the different termination mechanism, where the spiral wave is slightly pushed towards the inexcitable boundary. In contrast, at high LIs due to the immediate termination of the arrhythmia the number of pulses is less in both in silico and ex vivo. Similar behaviour is observed for termination time, in which both mean and median values show a decrease of termination time for increase of LIs, as it is shown in Fig.5.7-B and $\mathrm{B}^{\prime}$, in silico and ex vivo, respectively.

To calculate the required energy for arrhythmia termination we used the following equation:

$$
E=L I \times N \times P L \times A
$$

where LI is the light intensity, $\mathrm{N}$ is the number of pulses for termination, $\mathrm{PL}$ is the pulse length of illumination (in silico $\mathrm{PL}=33 \mathrm{~ms}$ and ex vivo $\mathrm{PL}=20 \mathrm{~ms}$ ), and $\mathrm{A}$ is the illuminated surface area (in silico $\mathrm{A}=625 \mathrm{~mm}^{2}$ and ex vivo $\mathrm{A}=274 \mathrm{~mm}^{2}$ ). Fig.5.8-A and $\mathrm{A}^{\prime}$ show the energy of a single pulse in silico and ex vivo, respectively. The two figures demonstrate energy increase at higher LI. The total amount of energy which is required to terminate arrhythmia in silico and ex vivo is plotted in Fig.5.8-B and $\mathrm{B}^{\prime}$, respectively. For the case of numerical study, the median values of the energy shows that when the illumination is below the threshold, where LI $\leqslant 40 \mu \mathrm{W} / \mathrm{mm}^{2}$, energy increases for increasing LI. The mean values show a similar trend. However, when the illumination is above the threshold, where LI is $>40 \mu \mathrm{W} / \mathrm{mm}^{2}$, the energy increases slightly and reaches to a steady state which is shown with both median and mean values. Fig.5.8-B' shows the total energy of ex vivo study. The trend of both median and mean values show that the energy required for arrhythmia termination is comparable throughout the LI $(3.1,103.5) \mu \mathrm{W} / \mathrm{mm}^{2}$ range.

\section{Discussion}

In cardiac research, control of cardiac arrhythmia with non-invasive clinical methods is still lacking $[4,52]$. Numerous theoretical and numerical studies are ongoing that study different approaches to control cardiac arrhythmia [63, 67, 74, 76]. Some of these studies have been validated in experiments [7-9], such as low-energy anti-fibrillation pacing (LEAP) [6]. 

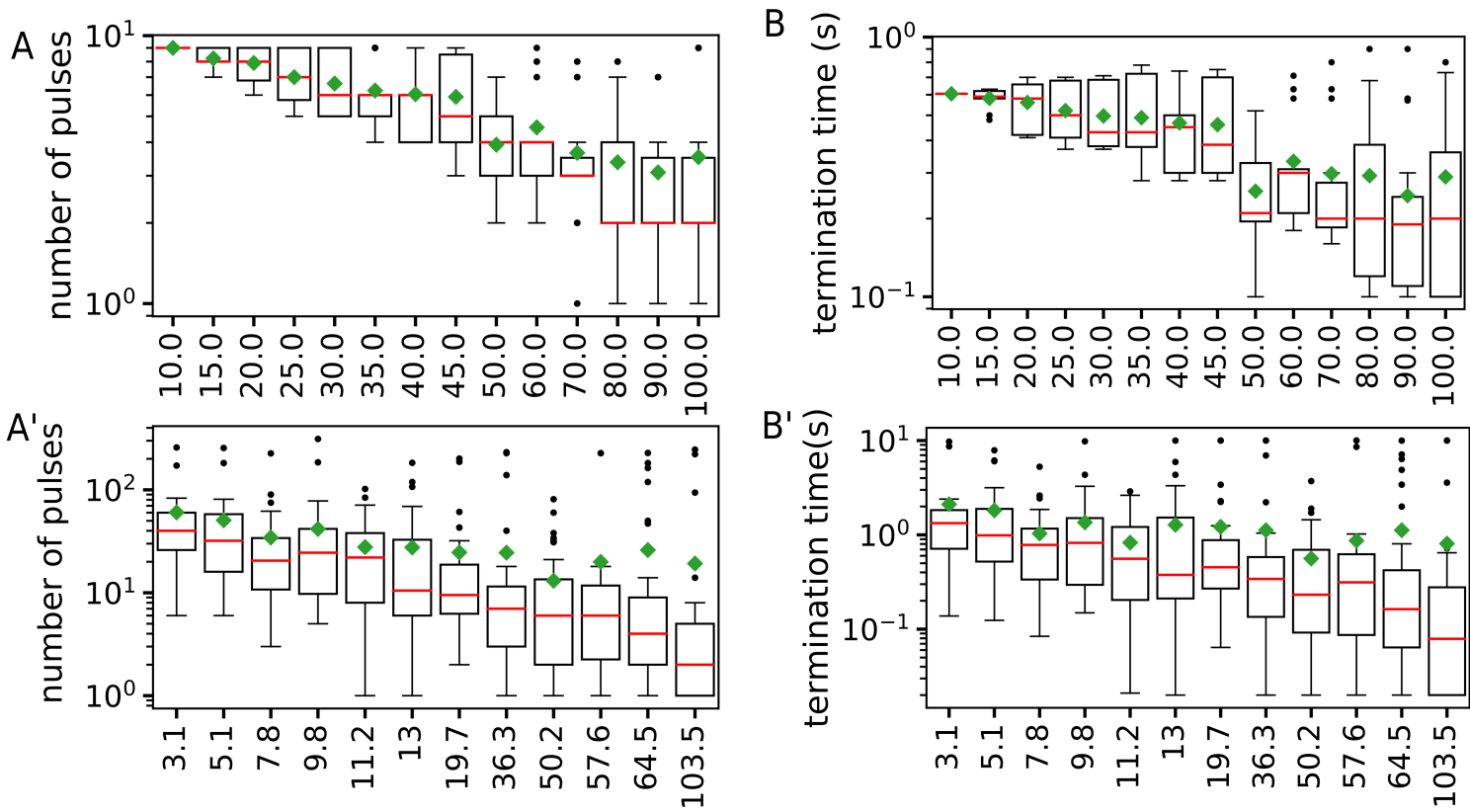

LI $\left(\mu \mathrm{W} / \mathrm{mm}^{2}\right)$

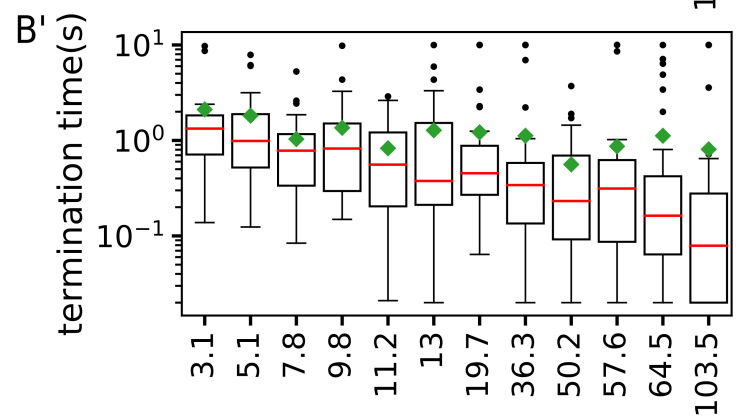

LI $\left(\mu \mathrm{W} / \mathrm{mm}^{2}\right)$

$\rightarrow$ mean

median

- outlier

Figure 5.7 - Number of pulses and time needed to terminate the arrhythmia in silico and ex vivo using resonant feedback pacing. A) Number of pulses to terminate arrhythmia at different $L I$ for $2 D$ simulation of mouse cardiac tissue. The median values (red line) and mean values (green almond marker) show a decrease in number of pulses for increasing LI. $\left.A^{\prime}\right)$ Shows an equivalent experimental study on intact mouse heart. It demonstrates a decrease in number of pulses for increasing LI. B) Illustrates termination time of arrhythmia in silico. Both median and mean values shows a decrease in termination time for increasing LI. $B^{\prime}$ ) Termination time of arrhythmia of ex vivo study. The median values illustrates a decrease of termination time for increasing $L I$.

In recent years, the possibility to control cardiac arrhythmia using resonant drift method has been studied extensively[92, 95, 208, 209]. Applying a uniform periodic external forcing with an appropriate frequency can drive the spiral wave towards inexcitable borders where it is terminated [199]. A numerical study on interaction of a spiral wave with boundaries shows that this interaction might dramatically effect the behaviour of drift and the spiral wave may be reflected from the boundary [96, 199]. Therefore any heterogeneities in the domain may have an effect on the spiral wave's dynamics $[83,196]$. In reality in a heterogeneous cardiac tissue resonant drift of a wave can be distorted and the core will not have a straight trajectory or when it reaches to an inexcitable boundary it may be repelled. Adjusting the stimulation frequency as the frequency of the spiral wave changes [202-204] is a solution to overcome the effects of inhomogeneities and the reflection by boundaries. V. Biktashev et al. [210] used feedback control which sends a stimuli when the excitation wavefront passes a particular recording point in the domain. S. Grill et al. [189] used light-responsive BZ 

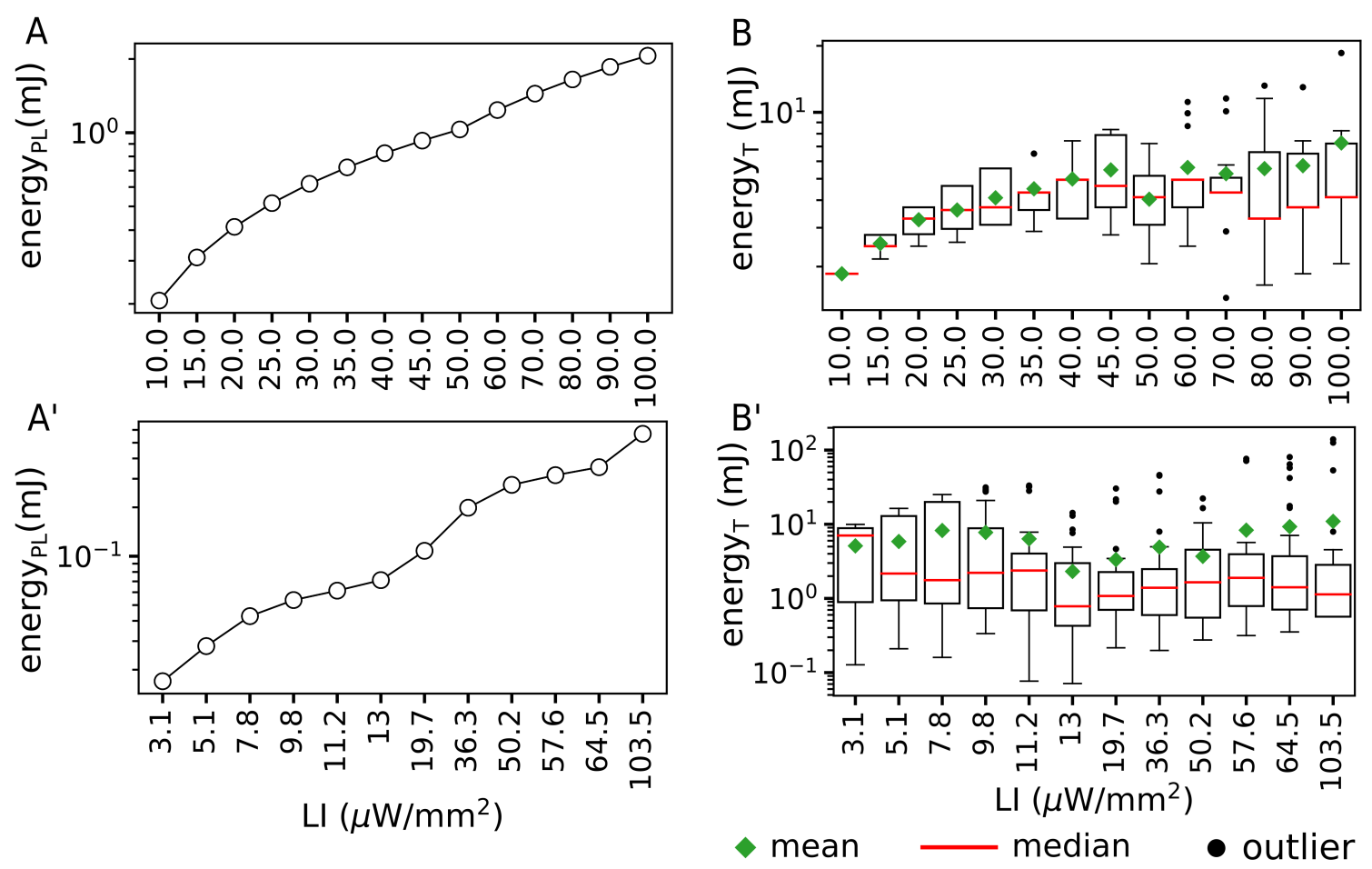

Figure 5.8 - Energy required to terminate arrhythmia in silico and ex vivo using resonant feedback pacing. A) Energy of a single light pulse with PL of $33 \mathrm{~ms}$ at different $L I$ in $2 D$ simulation of mouse cardiac tissue. It shows increase of energy for increasing LI. $\left.A^{\prime}\right)$ Shows an equivalent experimental study on intact mouse heart with the PL illumination of $20 \mathrm{~ms}$. It demonstrates increase of energy for increasing LI. B) Total energy required to terminate arrhythmia in silico. Both median (red line) and mean (red green almond marker) values shows an increase in energy at low $L I \leqslant 40 \mu \mathrm{W} / \mathrm{mm}^{2}$, but at high $L I>40 \mu \mathrm{W} / \mathrm{mm}^{2}$ the energy reaches a steady state. $B^{\prime}$ ) Energy required for termination of ex vivo study illustrates comparable mean and median values of energy over the entire range of $L I$.

reaction to study the feedback-controlled dynamics of meandering spiral waves. They showed when the measuring point is far from the unperturbed trajectory the spiral wave's tip trajectory is a large circle with the center of the measuring point.

In this study, we use optogenetics to control the dynamics of a spiral wave rotating in a $2 \mathrm{D}$ domain of the mouse ventricular heart coupled with the ChR2 model. We apply a periodic sub-threshold illumination at different $f_{p}$, which allows us to perturb the spiral wave so that the trajectory of the tip forms different meandering patterns such as hypocycloidal and epicycloidal. To induce a resonant drift, due to the decrease of $f_{s}$ at illumination, we applied a periodic sub-threshold pacing where $f_{p}$ was smaller than the initial $f_{s}$. Within a certain range of $f_{p}$, depending on the LI, we were able to induce a resonant drift where the spiral wave drifts to the inexcitable boundary and collides with it. In order to better control the spiral wave dynamics and to be able to steer in a desired direction, we have used the feedback method. We placed the 
Feedback and non-feedback control of cardiac arrhythmia

measuring electrode at different positions of the domain and showed an epicyloidal trajectory that forms around the measuring electrode. By placing the electrode at one corner of the domain, the spiral wave collides with the boundary on its way to form a circular trajectory around the measuring electrode.

Although the potential of the resonant drift technique has been proposed before as a low-energy defibrillation technique [95, 209, 211, 212], it has not been studied experimentally, yet. Optogenetics has shown its strong potential to study the dynamics of a spiral wave and gives us a deep understanding of its mechanistic properties, which is essential for the development of low-energy defibrillation techniques. The retention of electrical signal information during optical stimulation makes optogenetics a suitable tool to investigate the potential of the feedback method and experimentally compare it with a non-feedback method. Our experimental results on the intact mouse heart showed a high termination rate even at very low LIs $\left(\leqslant 11.2 \mu \mathrm{W} / \mathrm{mm}^{2}\right)$ for the case of feedback pacing. This could be due to the mechanism of feedback-induced resonant drift. In contrast, the termination rate for the non-feedback pacing case (overdrive pacing with a frequency of $30 \mathrm{~Hz}$ ) showed a decrease in efficiency in the range of LI $\leqslant$ $11.2 \mu \mathrm{W} / \mathrm{mm}^{2}$. In our simulation studies, we observed the comparable results where the termination rate of feedback pacing is higher than that of overdrive pacing at sub-threshold stimulation. Based on the numerical results, we tried to explain why there is a lower termination efficiency for the case of overdrive stimulation. This could be due to the inducing hypocycloid meandering pattern of the tip of the spiral wave during overdrive stimulation. This causes the tip of the spiral wave to rotate in a specific region without being directed towards an inexcitable boundary. In addition, overdrive pacing can trigger a wave break that puts the system into a more chaotic state [213]. Such a chaotic state is more difficult to control.

The energy required to terminate an arrhythmia in an intact mouse heart has been investigated in some studies [21-23, 126] using different structural and global illumination patterns. In the study by R. Q. Uribe et al. [23], they reported the delivery of a total energy of 3-153.6 mJ for a single global optical pulse at LI of 560 and 1100 $\mu \mathrm{W} / \mathrm{mm}^{2}$ and PL of 10,100 , and $1000 \mathrm{~ms}$. Our experimental results show that the energy required to terminate the arrhythmia ranges between mean values of 2-10 mJ for global illumination with $\mathrm{PL}=20 \mathrm{~ms}$ using the feedback method. The energy required for termination is comparable for the entire range of LI $(3.1,103.5) \mu \mathrm{W} / \mathrm{mm}^{2}$. Therefore, our results show that much less energy is required to control arrhythmias when a sequence of optical pulses resonantly triggers the arrhythmia compared to the single global optical illumination method.

Optical mapping is a valuable tool to show how electrical activity propagates in the heart during arrhythmias [20, 106]. Therefore, this technique can be used to show that the underlying mechanism of arrhythmia termination at very low LIs is resonant drift. 


\section{Chapter 6}

\section{Electric turbulence in cardiac tissue with sub-threshold modulation of excitability}

\subsection{Scientific goal and findings}

Cardiac arrhythmias are one of the main causes of death in industrialized countries. Interruptions in the propagating waves, commonly known as wave breaks, can lead to a deterioration in heart function if the heart is unable to pump blood, leading to a heart attack. In an arrhythmic heart, where one or more spiral waves propagate, wave breaks can occur due to inhomogeneities (regions of lower conductivity) naturally present in the heart tissue. A deeper understanding of the initiation and dynamics of wave breaks leads to progress in the therapy of arrhythmia.

In this study, optogenetic techniques and simulation were used to introduce a completely new mechanism of wave break initiation. A global constant sub-threshold illumination was applied to a spiral wave rotating in a $2 \mathrm{D}$ simulated, optogenetically modified human atrial tissue. The global periodic illumination leads to a change of the wave profile from front to back. A rapid recovery of the wave to the rear creates vulnerable windows for a sustained re-entry. This phenomenon occurs in the parameter regions where the restitution characteristics have a slope less than one.

To verify the generic nature of this mechanism of wave break initiation, I repeated the study in another 2D ionic model representing the ventricular tissue of a neonatal mouse heart.

\section{Manuscript Status}

The manuscript is under preparation 
Electric turbulence in cardiac tissue with sub-threshold modulation of excitability

\section{Author Contributions}

- Rupamanjari Majumder designed the research, performed the numerical simulations, analyzed the numerical data, and wrote the manuscript

- Sayedeh Hussaini validated the phenomenon in a different numerical model, analyzed the numerical data, and wrote the manuscript

- Vladimir Zykov designed the research, edited the manuscript

- Stefan Luther designed the research, edited the manuscript

- Eberhard Bodenschatz designed the research, edited the manuscript

\subsubsection{Manuscript}




\title{
Electric turbulence in cardiac tissue with subthreshold modulation of excitability.
}

\author{
Rupamanjari Majumder, Sayedeh Hussaini, ${ }^{*}$ Vladimir S. Zykov, Stefan Luther, ${ }^{*}$ and Eberhard Bodenschatz ${ }^{\dagger}$ \\ Max Planck Institute for Dynamics and Self-Organization, Göttingen, Germany
}

(Dated: December 17, 2020)

\begin{abstract}
Electric-turbulence in the heart is associated with complex spatial-temporal dynamics of nonlinear excitation waves. This life-threatening dynamical state is initiated by wave breaks and maintained by vortex-like rotating waves. However, the dynamic instabilities leading to the onset and perpetuation of this life-threatening state remain largely elusive. Two key questions are: 1) What determines the potential of a wave break to initiate re-entry? and 2) How do these breaks evolve such that the system is able to maintain spatiotemporally chaotic electrical activity? Here we show that subthreshold perturbations to the membrane voltage in spatially extended cardiac tissue lead to the development of wave breaks. Using a numerical optogenetics model of diseased human atrial tissue we find that these wave breaks occur even when the standard markers of vulnerability are absent. This contradicts the common understanding that only supra-threshold perturbations can induce arrhythmias. Progress in the treatment and therapy of cardiac arrhythmias requires a detailed understanding of the triggers and dynamics of these wave breaks. We therefore conclude that our results are of central importance for the further development of treatment strategies for cardiac defibrillation.
\end{abstract}

\section{INTRODUCTION}

Spiral waves occur as short- or long-lived transients in various natural excitable systems [1-11]. In the heart they appear as abnormal electrical waves which are the basis of fatal cardiac arrhythmias (tachycardia and fibrillation) [12]. Regardless of the type of medium that sustains these waves, spirals exhibit some common dynamic features, such as drift [13], meander [14, 15], anchoring [16-19], detachment [20-23] and breakup [24]. Spiral wave breakup or turbulence is of particular interest to cardiac researchers because it is associated with fibrillation, the most common precursor of stroke and sudden death. In particular, the mechanisms underlying the occurrence of spiral wave turbulence in heart tissue are extensively investigated because of their clinical significance and possible implications [25].

The tendency of a spiral to break up in an excitable medium is critically determined by the medium's excitability. Spatiotemporal modulation of the excitability of the medium can change the dynamics of spiral waves. Depending on the amplitude, frequency, degree of synchronization and the spatial scale of modulation of excitability, phenomena such as drift [26, 27], deformation [28], block [28], meander [27, 29], breakup [28, 30] and suppression of spiral waves [31] are known to occur. In experiments, however, excitability is extremely difficult to control in real time and in a reversible manner. Thanks to optogenetics, this has recently become possible to a considerable extent. Optogenetics has revolutionized cardiac research by successfully demonstrat-

\footnotetext{
* Institute for Dynamics of Complex Systems, University of Göttingen, D-37073 Göttingen, Germany

$\dagger$ Laboratory of Atomic and Solid-State Physics and Sibley School of Mechanical and Aerospace Engineering, Cornell University, Ithaca, New York 14853, USA
}

ing supposedly pain-free real-time spatio-temporal control of spiral wave dynamics in cardiac tissue [32-37]. With this tool one can regulate the flow of current across cell membranes to force these cell to trigger or suppress action potentials [38]. Although there is an extensive literature cataloguing the advantages and disadvantages of using optogenetics at supra-threshold light intensities (i.e. intensities capable of stimulating action potentials in individual cells or inducing waves in extended media), very little is known about the functionality and properties of the tool in the 'sub-threshold' régime. While some studies show that supra-threshold disturbances of spiral waves can cause wave breaks [39], the corresponding response at sub-threshold stimulation level remains incompletely explored.

An interesting study by Park et al. [40] demonstrates the possibility to achieve prolongation of the action potential duration (APD) in optogenetically modified cardiomyocytes by applying subthreshold optical stimuli. In particular, they show that optical pulses can prolong APD in a graded manner, thereby allowing one to 'scuplt' the action potential (AP) morphology in general. In another study by Burton et al. [41], the authors demonstrate that application of a subthreshold light pulse can lead to an increase in conduction velocity of a propagating wave. However, this increase only occurs in the speed of the first wave in a wave train. The reason may be attributed to the current-voltage characteristics of the Channelrhodopsin current, which displays an initial spike, followed by saturation at a decreased level, when the optogenetically-modified cell is continuously exposed to light.

In this study we demonstrate a heretofore unknown mechanism of wave break initiation by sub-threshold stimulation of excitable cardiac tissue. In two dimensions (2D) we observe that global periodic pulsing of a domain containing human atrial cardiomyocytes, with high subthreshold perturbations to the membrane voltage, lead 


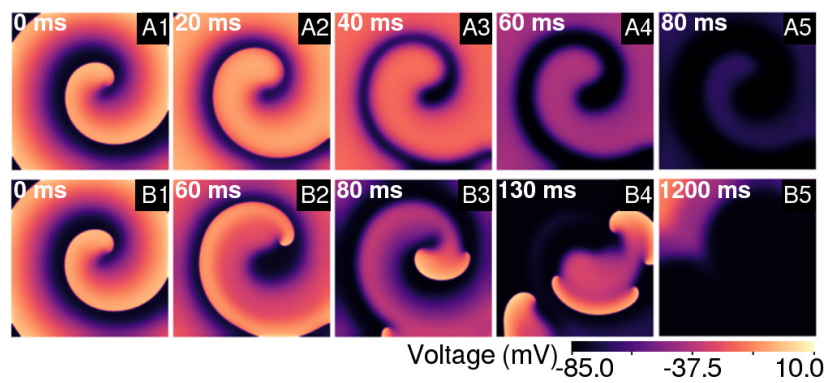

FIG. 1. Spiral wave dynamics in 2D human atrial tissue with subthreshold electrical perturbations. A1-A5 demonstrate synchronization-mediated termination of a single spiral wave when stimulated at $5.0 \mathrm{~Hz}$ with a globally-applied subthreshold electrical stimulus of $115 \mathrm{pA} / \mathrm{pF}$ and pulse duration 200 ms. B1-B5 demonstrate the formation of wavebreaks when the same domain is paced at $20 \mathrm{~Hz}$ with pulses of duration 10 ms.

to the development of wave breaks by "conditioning" of the wavelength. Such conditioning involves modulating the distribution of excitation along an arm of the intact spiral, which prompts the creation of excitation windows for wave break and re-entry. This observation is crucial for the planning and development of defibrillation strategies, as it provides a scenario in which the strategy can backfire and promote fibrillation.

\section{RESULTS}

The real heart is three-dimensional (3D) and consists of heterogeneous tissue with an organised fibre structure. In classical defibrillation, when a large amplitude electric field is applied across the heart, the heterogeneities behave like virtual electrodes that help with defibrillation by generating currents to ensure complete electrical synchronisation of the heart wall [25]. However, applying a high-voltage shock to the heart over a long period of time can have adverse effects on the tissue. Thus low energy methods are considered. However, virtual electrodes cannot be established with low energy, which makes low energy defibrillation a challenging problem. Intensive research is being carried out to develop defibrillation techniques that work with low energy and/or pulse trains as opposed to continuous application of the shock. In a previous study, Sridhar et al. [42] demonstrated in 2D that global synchronization-mediated spiral wave termination could also be achieved with low amplitude (subthreshold) electrical stimulation, applied for a duration of $\mathcal{O}(A P D)$. We observed that by modifying their technique to apply bursts of low amplitude electrical stimuli (where the duration of each burst is $\mathcal{O}(0.1 A P D)$ ), one can trigger the occurrence of wave breaks, as opposed to termination. These breaks appear in a very narrow parameter régime and are mostly non-sustained as the activity tends to selfterminate within a few cycles. These results are shown in Fig. 1. In order to understand how wavebreaks occur with subthreshold perturbation, we involved the use of optogenetics which allowed us to investigate wave dynamics in this system in a more subtle and controllable manner.

Under the influence of a constant, uniform global illumination, we found that an increase in the applied light intensity (quantified as the irradiance, $E_{e}$ ) led to an increase in the core size of the meandering spiral in two dimensions (2D), while preserving the general shape of the tip trajectory (Fig. 2A,B and D). The dominant frequencies of rotation of the spiral decreased with increase in $E_{e}$ as illustrated in Fig. 2C. Note that, both core size and dominant frequency of the spiral wave remained unaffected between $E_{e}=0.0$ and $0.085 \mathrm{~mW} / \mathrm{mm}^{2}$, beyond which value, these parameters exhibited sharp increase or decrease until $E_{e}=0.095 \mathrm{~mW} / \mathrm{mm}^{2}$, which marked the threshold of optical stimulation. However, replacement of the time-independent illumination with a periodic perturbation led to the emergence of interesting dynamical effects. In particular, uniform, global, time-periodic light pulses of $0.085 \mathrm{~mW} / \mathrm{mm}^{2} \leq E_{e}<0.095 \mathrm{~mW} / \mathrm{mm}^{2}$ led to the initiation of wave breaks, as shown in Fig. $2 \mathrm{E}$, visibly analogous to those observed with subthreshold electrical stimulation. These breaks appeared to occur right at the beginning of the 'dark' phase of the applied stimulus, i.e., when the light was turned off in a pacing cycle. Application of high subthreshold perturbations to membrane voltage led to the spontaneous emergence of conduction blocks within the domain (see bold white lines indicated by green arrows in Fig. 2E), which seemed to promote wave break initiation. For the full sequence of events leading to the incidence of wave breaks, see Video S1 of the SI Appendix.

To investigate the cause for the appearance of wave blocks in a system that is known to promote supernormal velocities [41], we first studied the influence of light on the dispersion of APD and CV, i.e. the restitution properties of the system. Our results, as shown in Fig. 3A and $\mathrm{B}$, pointed to a strange anomaly: Both the APD and $\mathrm{CV}$ restitution curves for different irradiance values appeared to have gentle slopes $(<<1.0)$ without spatial dispersion, which is fascinating because this type of wave break intiation is typically associated with steep restitution curves (slope $>1.0$ ), or dispersion in restitution properties within a system (contrary to our case), or in the presence of local ionic heterogeneities (as in case of ischemia) $[43,44]$ or in domains with progressively degrading excitability, which results in the gradual flatenning of the CV restitution curve with reduced mean CV, characteristic of type-II ventricular fibrillation. In addition, constant application of light to the domain resulted in supernormal CVs, which was in direct conflict with our observation of the emergence of lines of propagation block, just prior to the occurrence of the break.

Next, we tried to reproduce the appearance of wave blocks in a reduced system, i.e. pseudo-1D, using a rectangular simulation domain containing $512 \times 10$ grid 

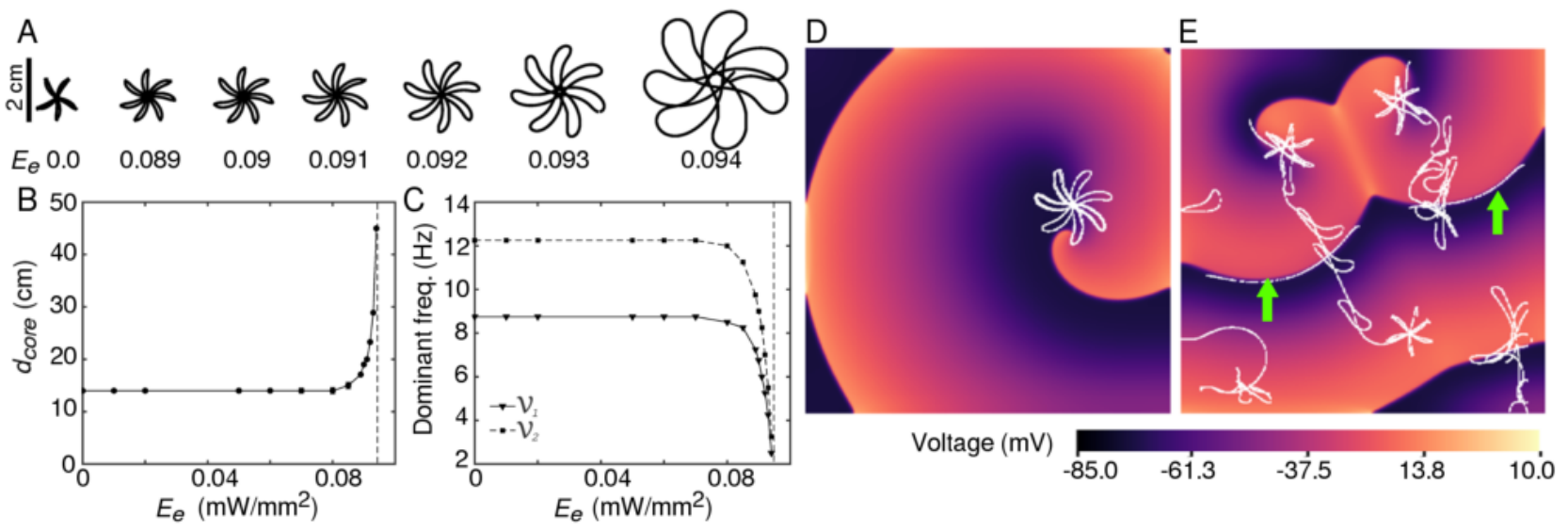

FIG. 2. Spiral wave dynamics in optogenetically-modified 2D human atrial tissue with uniform global subthreshold illumination. A) Tip trajectory of the spiral at different irradiance values $\left(E_{e}\right)$. B) Increase in diameter $\left(d_{\text {core }}\right)$ of the spiral core with increase in $E_{e}$. Here $d_{\text {core }}$ measures the diameter of the (outer) circle that just encloses the tip trajectory. C) Decrease in the dominant (first and second fundamental) frequencies of the spiral with increase in $E_{e}$. D) Trajectory of the spiral wave at constant, uniform, global illumination at $E_{e}=0.092 \mathrm{~mW} / \mathrm{mm}^{2}$. E) Break up of the spiral wave at the same $E_{e}$, when the light is applied as a periodic perturbation at a frequency of $1.0 \mathrm{~Hz}$. Green arrows point to the lines of the conduction block (shown in bold white). In $\mathrm{B}$ and $\mathrm{C}$, the dashed line at $E_{e}=0.095 \mathrm{~mW} / \mathrm{mm}^{2}$ marks the limit of subthreshold stimulation.

points. We applied high frequency electrical stimulation to the left edge of the domain (Fig. 4A), while illuminating the entire domain uniformly, in a time-periodic manner. In the absence of illumination, each applied stimulus resulted in the initiation of a new wave that propagated uninterruptedly to the right edge of the domain (Fig. 4A). However, in the presence of an optical stimulation with $E_{e} \geq 0.09 \mathrm{~mW} / \mathrm{mm}^{2}$ and low frequency, i.e., $<2.0 \mathrm{~Hz}$ we observed a random blocking of the stimulated waves, always at the site of stimulation (Fig. 4B). Since waveblock at the site of stimulation can occur due to various factors such as loss of capture, effect of CV restitution, interaction between new wavefront and slow waveback of the previous wave etc., our results remained inconclusive.
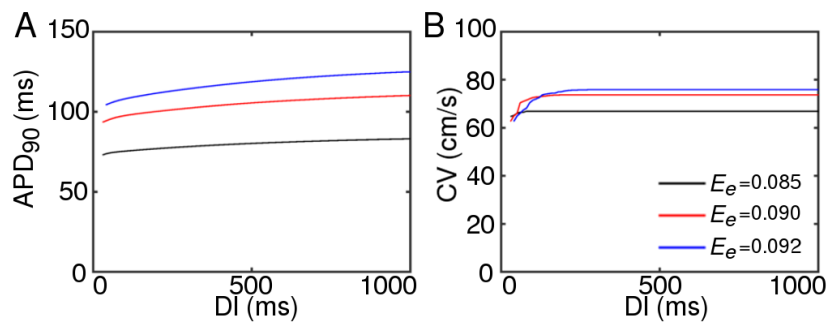

FIG. 3. A) $\mathrm{APD}_{90}$ and $\left.\mathrm{B}\right) \mathrm{CV}$ restitution curves at three different irradiance levels: $E_{e}=0.085 \mathrm{~mW} / \mathrm{mm}^{2}$ (black), $E_{e}$ $=0.09 \mathrm{~mW} / \mathrm{mm}^{2}$ (red) and $E_{e}=0.092 \mathrm{~mW} / \mathrm{mm}^{2}$ (blue). In each case, illumination was constant, uniform and global. The slopes of the $\mathrm{APD}_{90}$ restitution curves are 0.05, 0.07 and 0.071 for $E_{e}=0.085 \mathrm{~mW} / \mathrm{mm}^{2}, 0.09 \mathrm{~mW} / \mathrm{mm}^{2}$ and 0.092 $\mathrm{mW} / \mathrm{mm}^{2}$, respectively. Note that, CV restitution was studied here by looking only at the wavefront (and not the wave back) of the propagating waves.

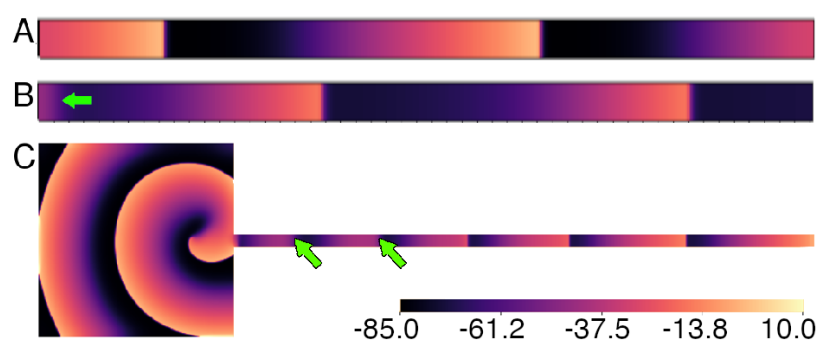

FIG. 4. A) Right propagating plane waves in a pseudo-1D domain containing $512 \times 10$ grid points, electrically paced at $11.5 \mathrm{~Hz}$ from the left edge of the domain in the absence of illumination. Each stimulus results in the generation of a new propagating wave. B) Wave block at the pacing site when the simulation in (A) is performed in the presence of a uniform global time-periodic illumination, at $1.0 \mathrm{~Hz}$ temporal frequency and and $\mathrm{LI}=0.092 \mathrm{~mW} / \mathrm{mm}^{2}$. C) Wave block away from the site of pacing, in a pseudo- $1 \mathrm{D}$ channel $(1536 \times 30$ grid points $)$ connected to a $2 \mathrm{D}$ domain $(512 \times 512$ grid points $)$ that maintains a spiral. The spiral drives the electrical stimulation in the channel. Time-periodic light stimulation is only applied to the pseudo-1D extension of the 2D domain. Green arrows indicate the locations of the wave block.

In order to obtain waveblocks inside the domain, away from the site of stimulation, we shifted the region for optical perturbation to $98<\mathrm{x}<512$, and resumed periodic electrical pacing at $11.5 \mathrm{~Hz}$ (dominant frequency of the spiral wave in the absence of illumination), from the left end $(\mathrm{x}<4)$ of the stripe. Our studies showed that every stimulus was capable of producing a new wave that propagated all the way to the right end of the stripe without interruption, although 1D traces along the length of the 
stripe did give us signatures of the effect of subthreshold stimulation on the membrane potential in the illuminated region. Thus, clearly, the $1 \mathrm{D}$ reduction of our $2 \mathrm{D}$ system was not optimised for wave block visualisation. To overcome this challenge, keeping in mind that the spiral in our 2D system was actually a meandering one, rotating quasi- periodically with 2 dominant frequencies, we chose to use the spiral in the $2 \mathrm{D}$ domain to drive the electrical activity in the stripe domain, while applying the optical perturbation only to the stripe domain. This had no direct bearing on the dynamics of the spiral wave itself, but was used as a driver of the electrical activity in the stripe domain, equivalent to the spiral in the $2 \mathrm{D}$ domain. Thus, we constructed a hybrid pseudo-1D equivalent of our original 2 D system by connecting a $2048 \times 30$ cell grid (pseudo-1D) to our 2D domain, which contained $512 \times 512$ grid points. We initiated a spiral in the $2 \mathrm{D}$ domain. The waves emitted from the spiral core were able to move into the pseudo-1D lattice and thereby control the frequency of the applied electrical stimulation. We applied uniform global optical stimulation at $E_{e}=0.092 \mathrm{~mW} / \mathrm{mm}^{2}$ and frequency $1.0 \mathrm{~Hz}$ only to the pseudo-1D domain, leaving the $2 \mathrm{D}$ domain unaffected by light. This setup allowed us to observe the formation of conduction blocks within the pseudo-1D domain, i.e. away from the stimulation site, over a wide range of frequencies of the applied light stimulation (Fig. 4C, and Video S2 of the SI Appendix).

In addition to the disappearance of some of the waves that had propagated into the pseudo-1D domain over a certain distance, we observed spatio-temporal oscillation in the wavelength of successive waves. To understand the basis for these oscillations and to correlate to the appearance of the conduction block, we studied the effects of sub-threshold illumination on a single heart cell when exposed to light at different phases of an electrically invoked AP (Fig. 5). Our studies demonstrate a phasedependent response of $A P D_{90}$. The actual value of the $A P D$ increased or decreased by an amount that was determined by the level of repolarization of the membrane at the beginning of the stimulation (see Fig. 5(A-F)). The applied light stimulus caused maximum increase in $A P D_{90}$ when it was applied at a membrane repolarization level of $\approx 64 \%$ (at the excitation threshold of the cardiac cell). Further repolarization of the membrane caused $A P D_{90}$ to decrease below its unperturbed value, reaching a minimum $(\approx 2 \%$ decrrease $)$ at a membrane repolarization level of $\approx 90 \%$, i.e., at the start of the refractory period in an unperturbed cell (Fig. 5G).

A direct consequence of this differential response of the cell membrane to light applied at different phases of the $\mathrm{AP}$ is a phase-dependent delay of recovery, that is introduced along the length of a wave, in extended media. Such a delay leads to differences in CV along a wavelength. Especially in the presence of light, the wavefront propagates at supernormal speeds while the waveback slows down. This results in $(i)$ prolongation of the wavelength with a gradual change in the shape of wave profile and $(i i)$ blocking of the following wave by the preceding one. When light is applied to a propagating wave, cells
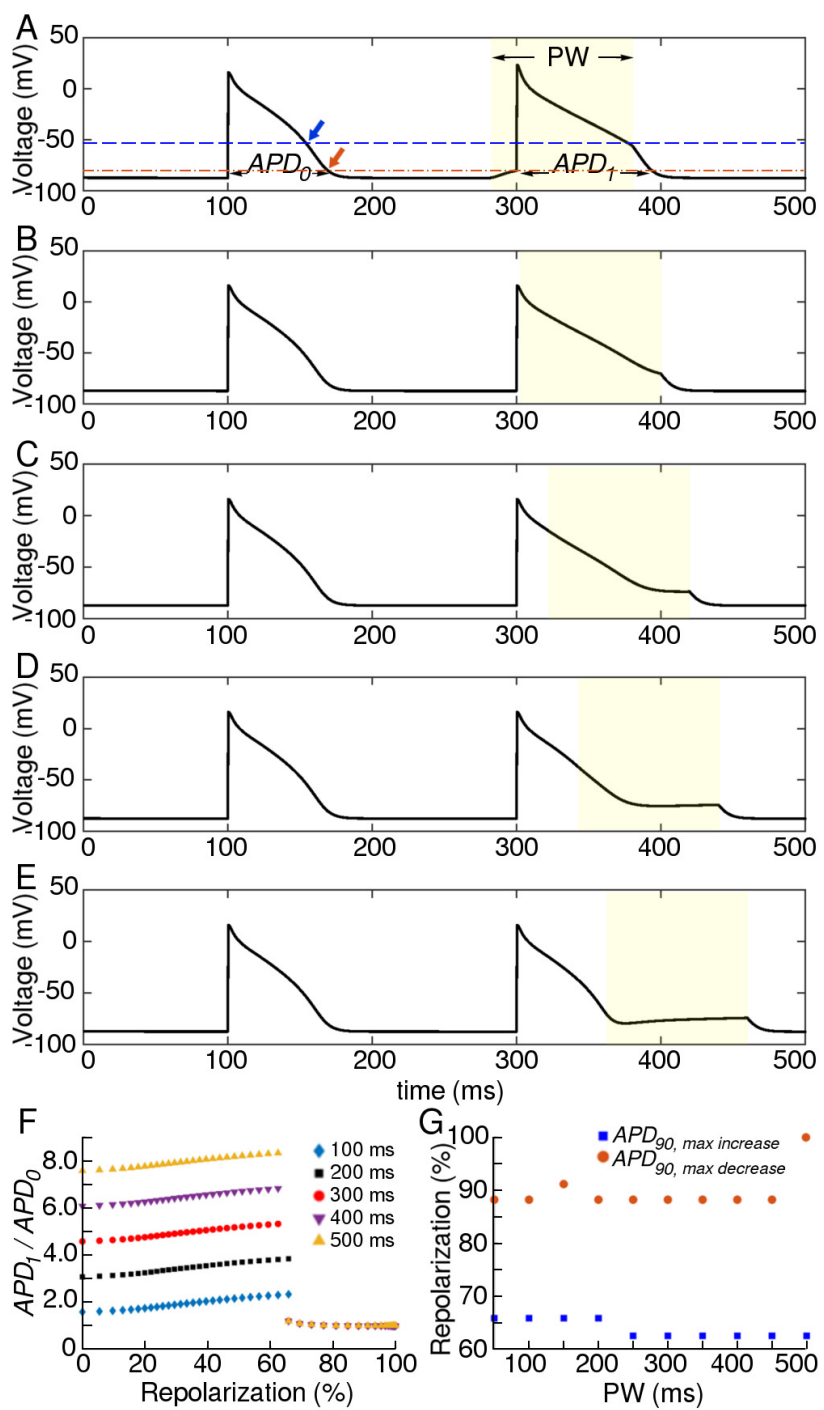

FIG. 5. (A-E) Effect of applying a light pulse (0.092 $\mathrm{mW} / \mathrm{mm}^{2}, 100 \mathrm{~ms}$ ) to different phases of an action potential (AP). Filled yellow rectangles mark the time (PW) when the light is applied. $A P D_{0}$ and $A P D_{1}$ refer to the $A P D_{90}$ values of the unperturbed and perturbed APs, respectively. (F) quantifies the findings in (A-E) in terms of the percentage of repolarization of the cell membrane at the start of application of the light stimulus, for different PWs. Here, Repolarization $=0 \%$ indicates that the membrane is fully depolarized, i.e., the AP is at the end of its upstroke phase. (G) shows the percentage repolarization of the membrane voltage at the start of application of the light stimulus that causes maximum increase (blue) or decrease (brown) in $A P D_{90}$ at each PW. The dashed blue and brown lines on (A) correlate the findings in $(\mathrm{G})$ to real values of the membrane voltage. The blue arrow in (A) indicates where the light stimulus should be applied in order to achieve maximum increase in $A P D_{90}$, whereas the corresponding brown arrow indicates the same for obtaining a maximum decrease in $A P D_{90}$ below normal level. 
located at increasing distances from the wavefront towards the waveback, receive light at progressively shifted phases of their AP (Fig. 5A to E). This results in a gradual decrease in the rate of recovery of the cells in between the wavefront and the waveback, thereby changing spatial profile of the wave, to give it a pinched appearance. (Fig. 6A-C). Instead of the constant, uniform, global illumination, if the light is now applied periodically in time, the pinched wavelength gets the opportunity to return to its original state during the light-off period (Fig. 6D-F). This explains the occurrence of spatiotemporal oscilla-
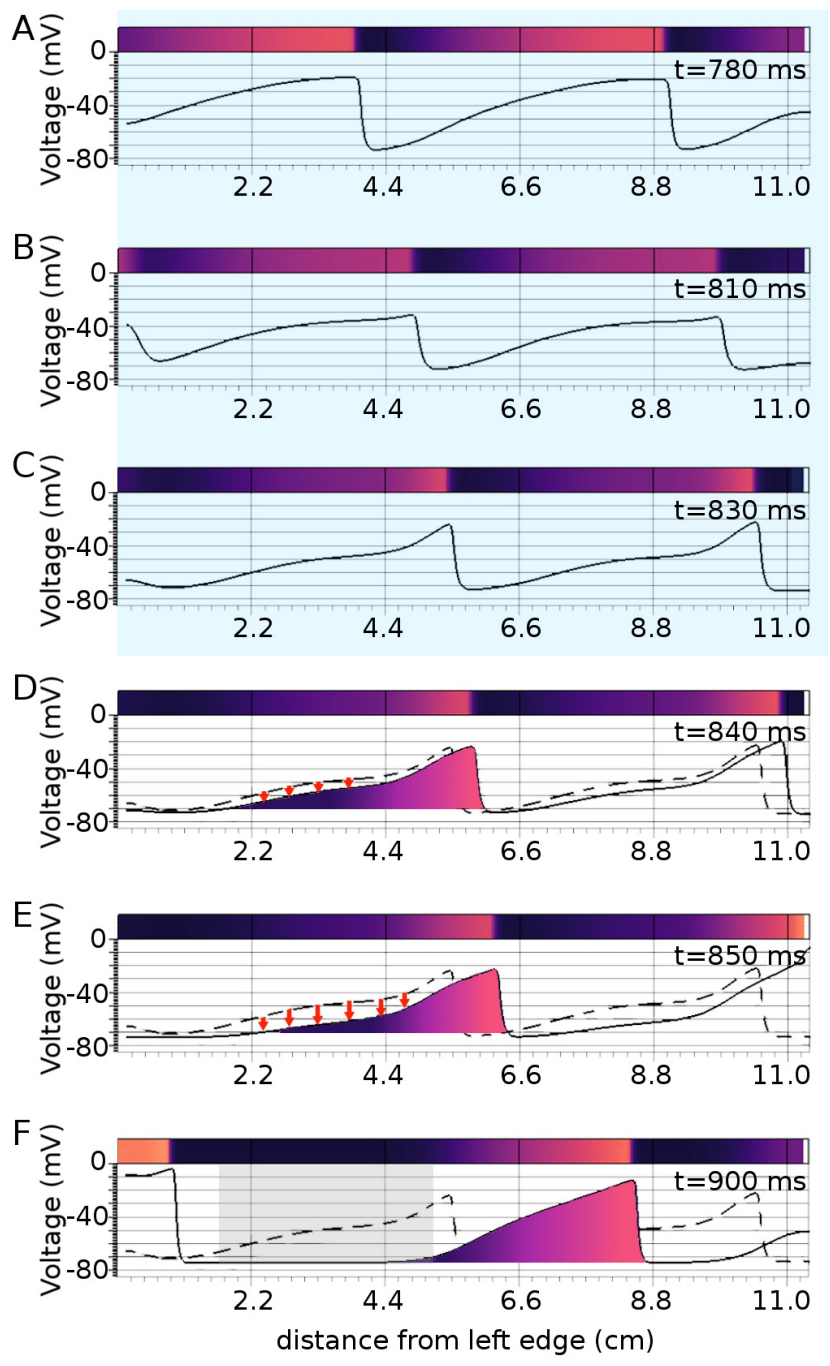

FIG. 6. (A-C) Effect of applying a $1000 \mathrm{~ms}, 0.092 \mathrm{~mW} / \mathrm{mm}^{2}$ light pulse to a plane wave propagating through a pseudo-1D domain containing $512 \times 10$ points. Voltage was measured along the line $\mathrm{y}=5$ within the domain. (D-F) Rapid recovery of the wave tail (indicated by bold red arrows) immediately after the light is turned off. The wave profile reshapes and widens the vulnerable window (indicated by the gray rectangle in F). The upper (colored) panel in each sub-figure shows the spatial profile of the wave as it propagates through the pseudo-1D domain.

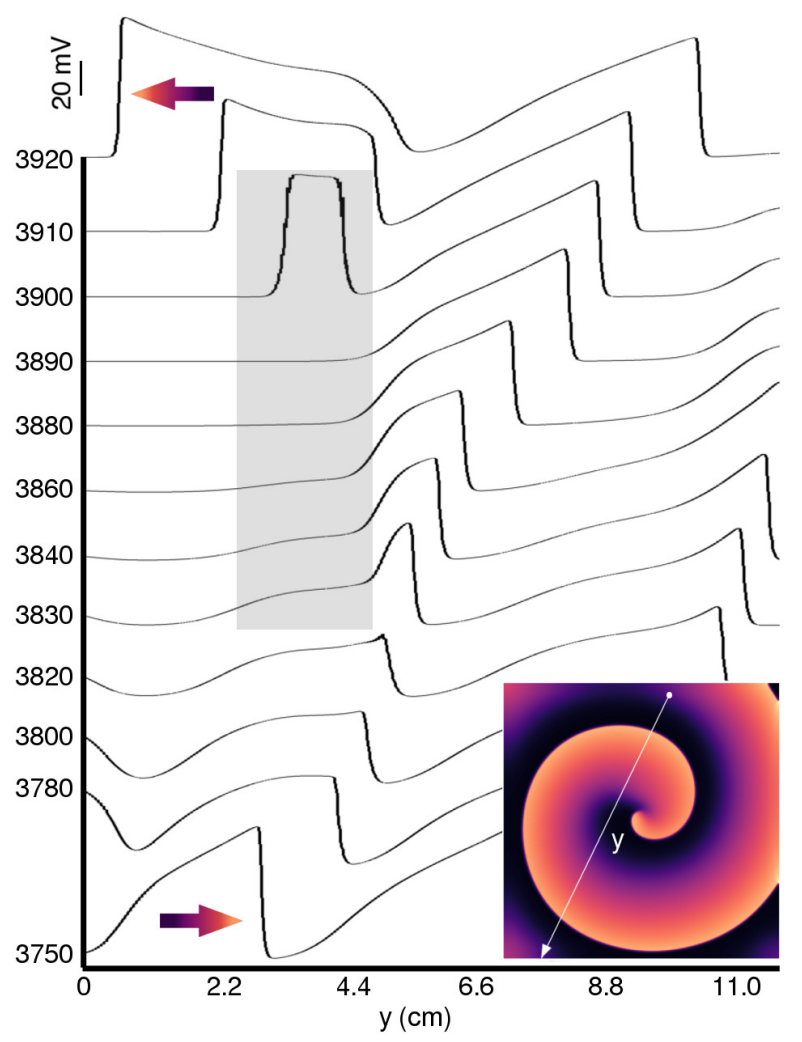

FIG. 7. Demonstration of the mechanism of spiral wave breakup in $2 \mathrm{D}$ using a series of $1 \mathrm{D}$ voltage traces measured along the vector $\mathbf{y}$ (indicated by the solid white arrow in the inset). The time stamp for each trace is shown on the left hand side of the vertical axis. The domain is illuminated uniformly and globally with $E_{e}=0.092 \mathrm{~mW} / \mathrm{mm}^{2}$, at a frequency of $1.0 \mathrm{~Hz}$. During this time, the CV of the rightpropagating wavefront increases beyond normal, whereas, that of the waveback decreases, resulting in a modulation of the spatial profile of the wave. A vulnerable window appears in the interval between $3830 \mathrm{~ms}$ and $3900 \mathrm{~ms}$ (indicated by filled gray rectangle). Re-excitation within the vulnerable window occurs, at $3900 \mathrm{~ms}$, to initiate a left-propagating wave.

tions in the wavelength of the propagating wave (in 1D) or a spiral arm (in 2D).

The higher the $E_{e}$, the stronger the conditioning of the wavelength, i.e. the wave appears more pinched. Immediately after the light is switched off, the differences between the recovery rates of the cells at different distances from the wavefront disappear. The cells on the wavefront that allowed propagation at supernormal CV recover slowly, allowing the wavefront to expand from its pinched state in a gradual manner. The cells on the wave back that were forcibly held at a sub-threshold voltage higher than the normal resting membrane potential (RMP) in the pinched state recover immediately, creating a window for re-excitation. In $2 \mathrm{D}$, this window allows re-entry, as shown in Fig. 7. The inset shows the spatial pattern of electrical activity in the domain, which takes 

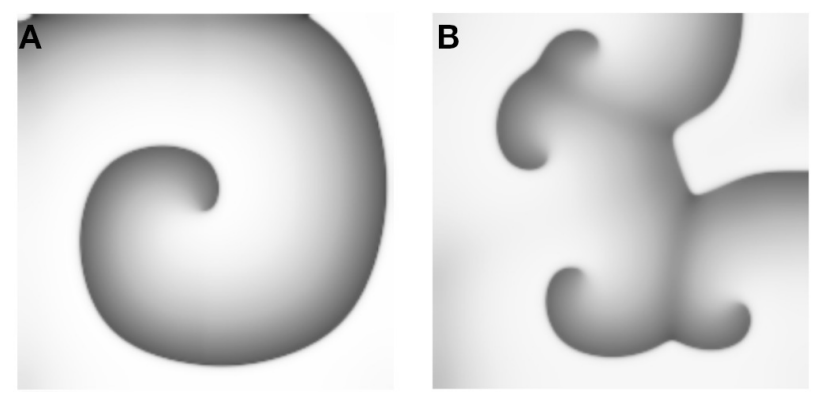

FIG. 8. Spiral wave breakup in the 2D model for optogenetically-modified neonatal mouse ventricular tissue. A) Spiral wave in the presence of uniform, global, constant illumination at $E_{e}=0.025 \mathrm{~mW} / \mathrm{mm}^{2}$. B) Break up of spiral waves in the presence of periodic optical stimulation at $4 \mathrm{~Hz}$, with a light pulse of duration $200 \mathrm{~ms}$.

up a spiral form. By tracing the voltage distribution along the solid white line (see Fig. 7, inset) in the direction of the arrow, we illustrate how a right-propagating wave is modulated over time with an optical perturbation $\left(E_{e}=0.092 \mathrm{~mW} / \mathrm{mm}^{2}\right.$, frequency $\left.1.0 \mathrm{~Hz}\right)$ to create a vulnerable window (marked with a filled gray rectangle). The wave break occurs at $\approx 3900 \mathrm{~ms}$, leading to re-excitation and reentry. This is marked by the reversal of wave direction.

Finally, to test the generic nature of this new mechanism of wave break initiation at stable parameter régimes, we repeated the study in another system, a 2D model of neonatal mouse ventricular tissue. At uniform global constant illumination with $\mathrm{LI}=0.025 \mathrm{~mW} / \mathrm{mm}^{2}$, a spiral wave rotated with no signatures of breakup (Fig. 8A). However, when stimulated periodically at 4 $\mathrm{Hz}$, with light of the same intensity and pulse length 200 ms, breakup similar to Fig. 2E was observed (Fig. 8B). A study of the restitution characteristics for this system, as presented in Ref.[45] (see Figures $5 \mathrm{~A}$ and B, therein), indicated that wave breaks occurred in a stable parameter régime, thus proving the robustness and restitution-independence of our proposed mechanism for wave breaks.

\section{DISCUSSION}

An intriguing question is: Would the choice of model parameters have an influence on wave break initiation, through a parameter-induced destabilizing effect (which may be characteristic of the cAF model used)? The answer is no. As such, the cAF model plays no specific role in determining the mechanism of wave break initiation and reentry. In Fig. 8 we demonstrate using a neonatal mouse ventricular model that such breaks can also occur in the ventricle. The reason for using the $\mathrm{cAF}$ model is because $(i)$ it makes more sense to 'defibrillate' a diseased condition rather than the healthy heart, where fibrillation is less likely to occur. We believe that any other parameter régime that supports fibrillation (atrial or ventricular type) or tachycardia, should also demonstrate this phenomenon, under the application of pulsed subthreshold light stimulation. ( $i i)$ The human atrial wall is super thin $(2-3 \mathrm{~mm})$ in contrast to the ventricles $(1.5 \mathrm{~cm})$, which makes the former a better candidate for optogenetic defibrillation, whose efficiency seems to rely on maximal illumination of bulk of the affected tissue. Researchers believe that the problem of poor penetration of visible light in cardiac tissue might not be so important in the human atria, because of its remarkably low wall thickness. Nevertheless, our studies reveal that one needs to be careful while designing the defibrillation protocol, as a series of globally illuminating light pulses in the suprathreshold regime, can, in fact trigger the formation of wave breaks underneath the surface, where illumination is subthreshold, and thereby lead to failure of defibrillation. Finally, (iii) from a technical perspective, we chose the cAF model over the healthy heart model because of the large computational costs associated with the latter (see Methods for details).

There are several theories regarding the factors and conditions that cause a spiral wave to break up. Of these, the strongest candidate is restitution (both APD and CV) in cardiac tissue [24]. Some studies argue that APD and CV restitution curves fundamentally characterize the wave dynamics in the heart as they reflect the mesoscopic effects of changes in ion currents and concentrations occurring at the cellular level [24]. Typically, a steep APD restitution curve (slope $\geq 1.0$ ) can drive the system via Hopf bifurcation to APD oscillations [46-48]. Such alternans promotes the development of functional conduction blocks because the propagation of a "long" action potential (AP) into a "short" AP region fails, thereby limiting the allowable diastolic interval (DI) [24]. Another proposed mechanism, again relating to the role of a steep APD restitution curve, suggests that the steepness of the curve is negatively correlated with the speed of the waveback in the presence of recovery gradients [49]. A gradient $\geq 1.0$ introduces a difference between the CVs of the propagating wavefront and back so that within a wave train the front of the following wave collides with the back of the preceding wave, resulting in a functional conduction block [47, 49-51]. Other mechanisms of wave break include the occurrence of a spatially non-synchronized type of APD oscillation, also known as spatially discordant APD alternans [52-55], the presence of a biphasic APD restitution curve [56, 57], effects of hysteresis [58-61] and Doppler shift through the trajectories of the spiral peaks [62].

A fascinating study by Fenton et al. [63] on wave break initiation in ischemic heart tissue shows that dynamic flatenning of the APD restitution curve (characterized by slope $<1.0$ ), results in an electrical 'memory' effect, which provokes a monotonic reduction in the excitability of cardiac tissue. Over time, the excitability becomes so low that a spiral breaks up close to its tip in the form of 
a 'voltage drop'. The study concludes that spiral breakup can be induced in parameter régimes with flat APD restitution as long as the dynamic effects of tissue memory and substantially large changes in basic cycle length are taken into account. Our study is consistent with the work of Fenton et al. in that wave breaks are observed at low APD and CV restitution slopes. However, we show that for a system with static APD and CV restitution curves, in the absence of a factor leading to a progressive reduction in tissue excitability and without explicit inclusion of the electrical memory effect, it is still possible to initiate wave breaks that develop into sustained re-entry.

Another study by Zemlin et al. [64] reports spiral wave break-up in parameter regimes characterized by strongly negative slopes of the APD restitution curve. However, as noted in their work, such break-up is often less pronounced than the break-up that occurs with positive slopes, mainly because break-up with negative restitution takes a fairly long time to mature, compared to break-up with positive restitution. The wave break initiation that we demonstrate in this article happens very fast, in most cases, requiring not more than 1-2 pulses.

Another study worth mentioning in this context is that of Banville and Gray [65], which demonstrates in experiments on the rabbit heart, the crucial role of the spatial distribution of APD- and CV restitution on the onset of alternans and fibrillation. However, in our study, we used a homogeneous domain with all cells identical, and subjected each cell to the same external conditions. Thus we did not have a dispersion of APD and CV restitution that could influence wave break initiation like in the case of [65]. Nevertheless, we observe the initiation of wave breaks, which leads us to rethink the underlying factors involved in initiating wave breaks in cardiac tissue under different conditions.

A rare, but quite efficient, mechanism of spiral wave break up involves supernormal excitability in certain domains. It is associated with a decrease in the excitation threshold with decreasing diastolic interval (DI). Typically, if it exists in a model, it promotes the development of conduction blocks through collisions between the rapidly advancing wave fronts and subsequent stacking at short DI, eventually leading to the initiation of wave breaks. Breakup occurs particularly close to the spiral tip. Supernormal CV can also lead to heterogeneity of refractoriness at shallow APD restitution, in which case scalloping occurs [24]. The mechanism of spiral wave break initiation that we report in the present article is somewhat similar to this phenomenon. With uniform global light perturbations, we are able to impose supernormal CVs on the propagating electrical activity. This results in the development of wave blocks within the domain that are visible as white lines in Fig. 2E. In our studies, the reentry occurs once the factor causing supernormality is withdrawn, i.e., the light perturbation is removed, allowing the wavefront and wave back to restore their normal velocities.
Finally, the effect of global subthreshold stimulation was studied in simple ionic models of cardiac tissue, in the context of electrical defibrillation. A particularly close phenomenon was reported by Sridhar and Sinha [42], who demonstrated the possibility to terminate electrical activity using a time-invariant global subthreshold electrical stimulation to achieve synchronization. Their study indicated that an electric pulse of length $\mathcal{O}(1 A P D)$ was sufficient to cause this effect. Our studies showed (data not presented) that if the stimulus in[42] was instead, applied at low frequency $(1-2 \mathrm{~Hz})$, using pulses of very short duration $(\mathcal{O}(0.1 A P D))$, then such perturbation could also initiate wavebreaks. However, such breaks occur within a very restricted parameter régime and critically rely on the frequency of the applied perturbation for sustenance, owing to the sensitive dependence of the response of the single cell AP morphology, on the phase of the AP at which the stimulus is applied.

Our study provides a clear example of a mechanism of wave break initiation and reentry that occurs when the slope of the APD restitution curve is less than 1.0, the CV restitution curve is relatively flat, and there is no factor causing dispersion of restitution properties within the domain, i.e., where the tissue does not exhibit any standard marker of vulnerability. As we demonstrate using a different animal model, the phenomenon is generic and does not depend on the selection of model used. The proposed mechanism serves as a candidate that has the potential to cause failure of low-amplitude defibrillation using pulsed global (electrical or optical) stimulation. We further believe that this mechanism is important in the context of optogenetic defibrillation, which is a long-term vision for many cardiac researchers, because of its promise for painless defibrillation. Since the human atrial wall is very thin, many researchers find it technically most optimal for optogenetic defibrillation. However, any step towards translation of this technique, necessitates the development of an in-depth understanding of not only the mechanisms that control, but also initiate, spiral waves. This holds true for low-energy defibrillation in general, where weak electric pulses, which are used to terminate spiral waves, can also potentially initiate them. The key to success lies in understanding and exploiting the mechanisms of creation and annihilation of spiral waves. Thus, our study makes an important contribution to the advancement of the field of cardiac optogenetics and arrhythmias, in general.

\section{METHODS}

Electrical activity in cardiac tissue was modeled using the following reaction-diffusion-type equation:

$$
\frac{d V}{d t}=\nabla \cdot \mathcal{D} \nabla V-\frac{I_{i o n}}{C_{m}}
$$

where $V$ represents the transmembrane potential (in $\mathrm{mV}$ ) developed across single cardiomyocytes, $C_{m}$ is the spe- 
cific capacitance (in $\mu \mathrm{F} / \mathrm{cm}^{2}$ ) of the cell membrane, $\mathcal{D}$ represents the diffusion coefficient for intercellular coupling, and $I_{i o n}$ represents the total ionic current produced by each individual cell. For human atrial tissue, $I_{i o n}$ was formulated according to the Courtemanche-RamirezNattel (CRN) model [66]. It is a sum of 12 ionic currents: fast $\mathrm{Na}^{+}\left(I_{\mathrm{Na}}\right)$, inward rectifier $K^{+}\left(I_{K 1}\right)$, transient outward $K^{+}\left(I_{t o}\right)$, ultra-rapid $K^{+}\left(I_{K u r}\right)$, rapid and slow delayed rectifier $K^{+}\left(I_{K r}\right.$ and $I_{K s}$, respectively), $\mathrm{Na}^{+}$and $\mathrm{Ca}^{2+}$ background $\left(I_{B N a}\right.$, and $\left.I_{C a L}\right), \mathrm{Na}^{+} / K^{+}$pump, $\mathrm{Ca}^{2+}$ pump $\left(\mathrm{I}_{\mathrm{pCa}}\right), \mathrm{Na}^{+} / \mathrm{Ca}^{2+}$ exchanger $\left(\mathrm{I}_{\mathrm{NaCa}}\right)$ and $L$-type $\mathrm{Ca}^{2+}$ current $\left(I_{C a L}\right)$. The parameters of the CRN model were adjusted to reproduce the action potential of the normal atrial working myocardium [67]. In particular, the maximum conductance for $I_{K 1}$, i.e., $G_{K 1}$ was changed from $0.09 \mathrm{nS} / \mathrm{pF}$ in the original model, to $0.117 \mathrm{nS} / \mathrm{pF}$, to yield a single cell action potential duration at $90 \%$ repolarization of the membrane potential $\left(\mathrm{APD}_{90}\right)$, of $284 \mathrm{~ms}$. A choice of $\mathcal{D}=0.0023 \mathrm{~cm}^{2} / \mathrm{ms}$, produced a conduction velocity (CV) of $69.75 \mathrm{~cm} / \mathrm{s}$ in the two-dimensional healthy tissue domain. In order to model the action potential during chronic atrial fibrillation (AF) remodelling, the maximal conductances of $I_{t o}$, and $I_{C a L}$ were reduced by $85 \%$, and $74 \%$, respectively, $G_{K 1}$ was increased by $250 \%$, the time constant for activation of $I_{C a L}$ was increased by $62 \%$, the activation curves for $I_{t o}$ and $I_{C a L}$ wer shifted by $+16 \mathrm{mV}$ and -5.4 $\mathrm{mV}$, respectively, while the inactivation curve for $I_{N a}$ was shifted by $+1.6 \mathrm{mV}$ [68-70]. These parameter adjustments reduced the wavelength (at $1 \mathrm{~Hz}$ electrical pacing) from $\simeq 18 \mathrm{~cm}$ in healthy tissue, to $\simeq 5 \mathrm{~cm}$, allowing us to fit the spiral into a smaller simulation domain $(512 \times 512$, as opposed to $2048 \times 2048$ ) without causing it to break up. This improved the computational cost-effectiveness by a factor of 20 . The spiral wave in our study meandered with a hypocycloidal tip trajectory, and survived for longer than $10 \mathrm{~s}$ of simulation time. For simulations with the CRN model, we used a time step $\delta t=0.02 \mathrm{~ms}$ and a grid spacing $\delta x=\delta y=0.022 \mathrm{~cm}$.

For the neonatal mouse ventricular model, $I_{\text {ion }}$ was expressed as a sum of 16 ionic currents, according to the Wang and Sobie [71]. These include, the fast $\mathrm{Na}^{+}$ current $\left(I_{\mathrm{Na}}\right)$, the background $\mathrm{Na}^{+}$and $\mathrm{Ca}^{2+}$ currents $\left(I_{N a b}\right.$ and $\left.I_{C a b}\right)$, the L-type and T-type $C a^{2+}$ currents ( $I_{C a L}$ and $\left.I_{C a T}\right)$, the $C a^{2+}$ pump current $\left(I_{p C a}\right)$, the $\mathrm{Na}^{+} / \mathrm{Ca}^{2+}$ exchanger $\left(I_{\mathrm{NaCa}}\right), \mathrm{Na}^{+} / \mathrm{K}^{+} \operatorname{pump}\left(I_{\mathrm{NaK}}\right)$, slow and fast components of the transient outward $K^{+}$ currents $\left(I_{K t o, f}\right.$ and $\left.I_{K t o, s}\right)$, slow and rapid delayed rectifier $K^{+}$currents $\left(I_{K s}\right.$ and $\left.I_{K r}\right)$, ultrarapid delayed rectifier $K^{+}$current $\left(I_{K u r}\right)$, sustained outward $K^{+}$current $\left(I_{K s s}\right)$, inward rectifier $\left(I_{K 1}\right)$, and the $\mathrm{Ca}^{2+}$-activated $\mathrm{Cl}^{-}$current $\left(I_{\mathrm{Cl}, \mathrm{Ca}}\right)$. We used $\mathcal{D}=0.00095 \mathrm{~cm} / \mathrm{ms}$, which led to a $\mathrm{CV}$ of $43.9 \mathrm{~cm} / \mathrm{s}$. The $2 \mathrm{D}$ simnulation domain contained $200 \times 200$ grid points.

In order to incorporate the effects of optogenetics, our models were combined with a 4-state model for voltageand light-sensitive Channelrhodopsin-2 [72], as described in Eqs. 2-16.

$$
\begin{gathered}
\frac{d V}{d t}=\mathcal{D} \nabla^{2} V-\frac{I_{\text {ion }}+I_{C h R 2}}{C_{m}} \\
I_{C h R 2}=g_{C h R 2} G(V)\left(O_{1}+\gamma O_{2}\right)\left(V-E_{C h R 2}\right) S_{c e l l} C_{m} \\
\frac{d C_{1}}{d t}=G_{r} C_{2}+G_{d 1} O_{1}-k_{1} C_{1} \\
\frac{d C_{2}}{d t}=G_{d 2} O_{2}-\left(k_{2}+G_{r}\right) C_{2} \\
\frac{d O_{1}}{d t}=k_{1} C_{1}-\left(G_{d 1}+e_{12}\right) O_{1}+e_{21} O_{2} \\
\frac{d O_{2}}{d t}=k_{2} C_{2}-\left(G_{d 2}+e_{21}\right) O_{2}+e_{12} O_{1} \\
O_{1}+O_{2}+C_{1}+C_{2}=1 \\
G(V)=10.6408-14.6408 \times \frac{\exp (-V / 42.7671)}{V}
\end{gathered}
$$

Here $O_{1}, O_{2}, C_{1}$ and $C_{2}$ represent the open and closed states of the ChR2 ion channel, $S_{\text {cell }}$ is the cell surface area, $k_{1}, k_{2}, G_{d 1}=0.1, G_{d 2}=0.05, G_{r}=0.004, e_{12}$, and $e_{21}$ are the kinetic parameters, described as follows:

$$
\begin{gathered}
k_{1}=0.8535 \times \text { Flux } \times p, \\
k_{2}=0.14 \times \text { Flux } \times p, \\
\text { Flux }=\left(0.00006 E_{e} \lambda w_{\text {loss }}\right), \\
\frac{\partial p}{\partial t}=\frac{S_{0}-p}{\tau_{C h R 2}}, \\
S_{0}=0.5\left(1+\tanh \left(120\left(E_{e}-0.1\right)\right)\right), \\
e_{12}=0.011+0.005 \times \log \left(E_{e} / 0.024\right), \\
e_{21}=0.008+0.004 \times \log \left(E_{e} / 0.024\right)
\end{gathered}
$$

where, $E_{e}$ is the irradiance, $\lambda$ is the wavelength of light used $(470 \mathrm{~nm})$, and $w_{\text {loss }}$ is the scaling factor for loss of photons. We used $g_{C h R 2}=0.17 \mathrm{mS} / \mathrm{cm}^{2}$ [34], and calculated $S_{\text {cell }}$ as area of a cylindrical cell of length = $100 \mu \mathrm{m}$ and diameter $=16 \mu \mathrm{m}$. For a detailed description of other parameters and their values, we refer the reader to [73]. We found that for human atrial tissue, $E_{e}<$ $0.095 \mathrm{~mW} / \mathrm{mm}^{2}$ were typically sub-threshold, meaning, they failed to trigger action potentials, whereas, higher light intensities successfully stimulated cells and initiated waves in extended media. In neonatal mouse, $E_{e}<0.03$ $\mathrm{mW} / \mathrm{mm}^{2}$ was considered sub-threshold. 


\section{ACKNOWLEDGEMENT}

This work was supported by the Max Planck Society and the German Center for Cardiovascular Research (DZHK).

\section{SI APPENDIX}

Video S1: Controlled break-up of spiral waves, induced by periodic sub-threshold optical stimulation, at frequency $1.0 \mathrm{~Hz}$. The trajectory of the spiral tip(s) is traced using white lines that fade over time. The filled blue circle below the voltage color-code (in $\mathrm{mV}$ ) in select frames indicates the application of uniform, global subthreshold illumination at $E_{e}=0.092 \mathrm{~mW} / \mathrm{mm}^{2}$, in these frames.

Video S2: Reduction of the $2 \mathrm{D}$ system to a hybrid $1 \mathrm{D}$ system, in which we combine a $2 \mathrm{D}$ simulation domain to a pseudo-1D outlet. The electrical stimulation in the pseudo-1D domain is driven by the unperturbed spiral wave in 2D. A uniform global periodic subthreshold stimulation (frequency: $1.0 \mathrm{~Hz}$ ) with light of intensity $0.092 \mathrm{~mW} / \mathrm{mm}^{2}$ is applied to the pseudo-1D domain. Propagation failure occurs well within the pseudo-1D domain for randomly selected waves. In addition, we observe oscillations in wavelength and modulation of the wave profile for waves propagating through the pseudo-1D domain.
[1] E. Pálsson and E. C. Cox, Origin and evolution of circular waves and spirals in dictyostelium discoideum territories, Proc Natl Acad Sci U S A 93, 1151 (1996).

[2] R. J. Field and M. Burger, eds., Oscillations and traveling waves in chemical systems. (Wiley, New York, 1985).

[3] A. M. Zhabotinsky, A history of chemical oscillations and waves, Chaos 1, 379 (1991).

[4] M. Bär, N. Gottschalk, M. Eiswirth, and G. Ertl, Spiral waves in a surface reaction: Model calculations, The Journal of Chemical Physics 100, 1202 (1994).

[5] V. Krinsky and H. Swinney, eds., Wave and Patterns in Biological and Chemical Excitable Media (NorthHolland, Amsterdam, 1991).

[6] R. Kapral and K. Showalter, eds., Chemical Waves and Patterns (Kluwer, Dordrecht, 1995).

[7] T. Lilienkamp and U. Parlitz, Terminating transient chaos in spatially extended systems, Chaos: An Interdisciplinary Journal of Nonlinear Science 30, 051108 (2020).

[8] T. Lilienkamp and U. Parlitz, Scaling behavior of the terminal transient phase., Physical review. E 98 2-1, 022215 (2018).

[9] T. Lilienkamp and U. Parlitz, Susceptibility of transient chimera states, Phys. Rev. E 102, 032219 (2020).

[10] M. Aron, S. Herzog, U. Parlitz, S. Luther, and T. Lilienkamp, Spontaneous termination of chaotic spiral wave dynamics in human cardiac ion channel models, PLOS ONE 14, 1 (2019).

[11] T. Lilienkamp and U. Parlitz, engTerminal transient phase of chaotic transients, Physical Review Letters 120, 094101 (2018).

[12] J. M. Davidenko, P. Kent, D. R. Chialvo, D. C. Michaels, and J. Jalife, Sustained vortex-like waves in normal isolated ventricular muscle, Proc. Natl. Acad. Sci. USA. 87, 8785 (1990)

[13] A. V. Panfilov, R. H. Keldermann, and M. P. Nash, Drift and breakup of spiral waves in reaction-diffusion-mechanics systems, Proceedings of the National Academy of Sciences 104, 7922 (2007), https://www.pnas.org/content/104/19/7922.full.pdf.

[14] N. F. Otani, A primary mechanism for spiral wave meandering, Chaos: An Interdisciplinary Journal of Nonlinear Science 12, 829 (2002).
[15] H. Zhang and A. Holden, Chaotic meander of spiral waves in the fitzhugh-nagumo system, Chaos, Solitons \& Fractals 5, 661 (1995), nonlinear Phenomena in Excitable Physiological Systems.

[16] O. Steinbock and S. C. Müller, Light-controlled anchoring of meandering spiral waves, Phys. Rev. E 47, 1506 (1993).

[17] P. Kuklik, J. J. Zebrowski, L. Szumowski, and P. Sanders, Anchoring of spiral waves by inhomogeneity of anisotropic conduction, Heart, Lung and Circulation 17, S7 (2008).

[18] P. Boily, V. G. LeBlanc, and E. Matsui, Spiral anchoring in media with multiple inhomogeneities: A dynamical system approach, Journal of Nonlinear Science 17, 399 (2007).

[19] D. Olmos, Reflection and attachment of spirals at obstacles for the fitzhugh-nagumo and beeler-reuter models, Phys. Rev. E 81, 041924 (2010).

[20] H. Kitahata and M. Tanaka, Mathematical approach to unpinning of spiral waves anchored to an obstacle with high-frequency pacing., Biophysics and physicobiology 15, 196 (2018).

[21] P. Porjai, M. Sutthiopad, J. Luengviriya, M. Phantu, S. C. Müller, and C. Luengviriya, Electrically forced unpinning of spiral waves from circular and rectangular obstacles, Chemical Physics Letters 660, 283 (2016).

[22] X. Feng, X. Gao, D.-B. Pan, B.-W. Li, and H. Zhang, Unpinning of rotating spiral waves in cardiac tissues by circularly polarized electric fields, Scientific Reports 4, 10.1038/srep04831 (2014).

[23] M. Tanaka, A. Isomura, M. Hörning, H. Kitahata, K. Agladze, and K. Yoshikawa, Unpinning of a spiral wave anchored around a circular obstacle by an external wave train: Common aspects of a chemical reaction and cardiomyocyte tissue, Chaos: An Interdisciplinary Journal of Nonlinear Science 19, 043114 (2009), https://doi.org/10.1063/1.3263167.

[24] F. H. Fenton, E. M. Cherry, H. M. Hastings, and S. J. Evans, Multiple mechanisms of spiral wave breakup in a model of cardiac electrical activity, Chaos: An Interdisciplinary Journal of Nonlinear Science 12, 852 (2002).

[25] S. Luther, F. Fenton, B. Kornreich, A. Squires, P. Bittihn, D. Hornung, M. Zabel, J. Flanders, A. Gladuli, 
L. Campoy, E. Cherry, G. Luther, G. Hasenfuss, V. Krinsky, A. Pumir, R. J. Gilmour, and E. Bodenschatz, Lowenergy control of electrical turbulence in the heart, Nature 475, 235 (2011).

[26] L. Xu, Z. Li, Z. Qu, and Z. Di, Resonance drifts of spiral waves on media of periodic excitability, Physical review. E, Statistical, nonlinear, and soft matter physics 85, 046216 (2012).

[27] S. Grill, V. S. Zykov, and S. C. Müller, Spiral wave dynamics under pulsatory modulation of excitability, The Journal of Physical Chemistry 100, 19082 (1996).

[28] J. Ramos, Dynamics of spiral waves in excitable media with local time-periodic modulation, Chaos, Solitons \& Fractals 13, 1383 (2002).

[29] O. Steinbock, V. Zykov, and S. Müller, Control of spiralwave dynamics in active media by periodic modulation of excitability, Nature 366, 322 (1993).

[30] A. Schrader, M. Braune, and H. Engel, Dynamics of spiral waves in excitable media subjected to external periodic forcing, Phys. Rev. E 52, 98 (1995).

[31] J. Ma, C.-N. Wang, J. Tang, and Y.-F. Xia, Suppression of the spiral wave and turbulence in the excitabilitymodulated media, International Journal of Theoretica Physics 48, 150 (2009).

[32] T. Bruegmann, D. Malan, M. Hesse, T. Beiert, C. Fuegemann, B. Fleischmann, and P. Sasse, Optogenetic control of heart muscle in vitro and in vivo, Nat Methods. 7, 897 (2010).

[33] C. Crocini, C. Ferrantini, R. Coppini, M. Scardigli, P. Yan, L. Loew, G. Smith, E. Cerbai, C. Poggesi, F. Pavone, and L. Sacconi, Optogenetics design of mechanistically-based stimulation patterns for cardiac defibrillation, Sci Rep. 6, 35628 (2016).

[34] T. Bruegmann, P. Boyle, C. Vogt, T. Karathanos, H. Arevalo, B. Fleischmann, N. Trayanova, and P. Sasse, Optogenetic defibrillation terminates ventricular arrhythmia in mouse hearts and human simulations, The Journal of Clinical Investigation 126, 3894 (2016).

[35] E. C. A. Nyns, A. Kip, C. I. Bart, J. J. Plomp, K. Zeppenfeld, M. Schalij, A. De Vries, and D. A. Pijnappels, Optogenetic termination of ventricular arrhythmias in the whole heart: towards biological cardiac rhythm management, Eur Heart J. 38, 2132 (2017).

[36] T. Bruegmann, T. Beiert, C. Vogt, J. Schrickel, and P. Sasse, Optogenetic termination of atrial fibrillation in mice, Cardiovasc Res. 114, 713 (2018).

[37] R. Majumder, I. Feola, A. S. Teplenin, A. A. de Vries, A. V. Panfilov, and D. A. Pijnappels, Optogenetics enables real-time spatiotemporal control over spiral wave dynamics in an excitable cardiac system, eLife 7, e41076 (2018).

[38] Z. Jia, V. Valiunas, Z. Lu, H. Bien, H. Liu, H. Wang, B. Rosati, P. Brink, I. Cohen, and E. Entcheva, Stimulating cardiac muscle by light: cardiac optogenetics by cell delivery, Circ Arrhythm Electrophysiol. 4, 753 (2011).

[39] I. Feola, L. Volkers, R. Majumder, A. Teplenin, M. J. Schalij, A. V. Panfilov, A. A. F. de Vries, and D. A. Pijnappels, Localized optogenetic targeting of rotors in atrial cardiomyocyte monolayers, Circ Arrhythm Electrophysiol. 10, e005591 (2017).

[40] S. Park, S. Lee, L. Tung, and D. Yue, Optical mapping of optogenetically shaped cardiac action potentials, Scientific reports 4, 6125 (2014).
[41] R. Burton, A. Klimas, C. Ambrosi, J. Tomek, A. Corbett, E. Entcheva, and G. Bub, Optical control of excitation waves in cardiac tissue, Nature photonics 9, 813 (2015).

[42] S. Sridhar and S. Sinha, Euro Physics Letters 92, 60006 (2010).

[43] T. Wu, S. Lin, J. Weiss, C. Ting, and P. Chen, Circulation 106, 1859 (2002)

[44] Y. Liu, H. Pak, S. Lamp, Y. Okuyama, H. Hayashi, T. Wu, J. Weiss, P. Chen, and S. Lin, J. Cardiovasc. Electrophysiol. 15, 1433 (2004).

[45] A. Mayer, P. Bittihn, and S. Luther, Complex restitution behavior and reentry in a cardiac tissue model for neonatal mice, Physiological Reports 5, e13449 (2017).

[46] J. B. Nolasco and R. W. Dahlen, A graphic method for the study of alternation in cardiac action potentials., Journal of Applied Physiology 25, 191 (1968).

[47] M. Courtemanche, L. Glass, and J. P. Keener, Instabilities of a propagating pulse in a ring of excitable media, Phys. Rev. Lett. 70, 2182 (1993).

[48] A. Karma, H. Levine, and X. Zou, Theory of pulse instabilities in electrophysiological models of excitable tissues, Physica D: Nonlinear Phenomena 73, 113 (1994).

[49] M. Courtemanche, Complex spiral wave dynamics in a spatially distributed ionic model of cardiac electrical activity, Chaos: An Interdisciplinary Journal of Nonlinear Science 6, 579 (1996).

[50] M. Courtemanche, J. P. Keener, and L. Glass, A delay equation representation of pulse circulation on a ring in excitable media, SIAM J. Appl. Math. 56, 119-142 (1996).

[51] M. Watanabe, F. Fenton, S. Evans, H. Hastings, and A. Karma, Mechanisms for discordant alternans, Journal of Cardiovascular Electrophysiology 12, 196 (2001), https://onlinelibrary.wiley.com/doi/pdf/10.1046/j.15408167.2001.00196.x.

[52] D. S. Rosenbaum, L. E. Jackson, J. M. Smith, H. Garan, J. N. Ruskin, and R. J. Cohen, Electrical alternans and vulnerability to ventricular arrhythmias, New England Journal of Medicine 330, 235 (1994).

[53] D. Sato, Y. Shiferaw, A. Garfinkel, J. N. Weiss, Z. Qu, and A. Karma, Spatially discordant alternans in cardiac tissue, Circulation Research 99, 520 (2006).

[54] H. Hayashi, Y. Shiferaw, D. Sato, M. Nihei, S. Lin, P. Chen, A. Garfinkel, J. Weiss, and Z. Qu, Dynamic origin of spatially discordant alternans in cardiac tissue., Biophysical journal 92, 448 (2007).

[55] R. Majumder, M. C. Engels, A. F. de Vries, Antoine, A. V. Panfilov, and A. Pijnappels, Daniel, Islands of spatially discordant apd alternans underlie arrhythmogenesis by promoting electrotonic dyssynchrony in models of fibrotic rat ventricular myocardium., Scientific reports 6 , 10.1038/srep24334 (2016).

[56] M. R. Franz, C. D. Swerdlow, L. B. Liem, and J. Schaefer, Cycle length dependence of human action potential duration in vivo. effects of single extrastimuli, sudden sustained rate acceleration and deceleration, and different steady-state frequencies., The Journal of Clinical Investigation 82, 972 (1988).

[57] P. Szigligeti, T. Bányász, J. Magyar, G. Szigeti, Z. Papp, A. Varró, and P. Nánási, Intracellular calcium and electrical restitution in mammalian cardiac cells, Acta Physiologica Scandinavica 163, 139 (1998), https://onlinelibrary.wiley.com/doi/pdf/10.1046/j.1365201X.1998.00362.x. 
[58] P. Lorente and J. Davidenko, Hysteresis phenomena in excitable cardiac tissuesa, Annals of the New York Academy of Sciences 591, 109 (1990).

[59] G. M. Hall, S. Bahar, and D. J. Gauthier, Prevalence of Rate-Dependent Behaviors in Cardiac Muscle, Physical Review Letters 82, 2995 (1999).

[60] A. R. Yehia, D. Jeandupeux, F. Alonso, and M. R. Guevara, Hysteresis and bistability in the direct transition from 1:1 to $2: 1$ rhythm in periodically driven single ventricular cells, Chaos: An Interdisciplinary Journal of Nonlinear Science 9, 916 (1999).

[61] R. A. Oliver, G. M. Hall, S. Bahar, W. Krassowska, P. D. Wolf, E. G. D. Tulloch, and D. J. Gauthier, Existence of bistability and correlation with arrhythmogenesis in paced sheep atria, Journal of Cardiovascular Electrophysiology 11, 797 (2000), https://onlinelibrary.wiley.com/doi/pdf/10.1111/j.15408167.2000.tb00051.x.

[62] O. Steinbock, J. Schütze, and S. C. Müller, Electric-fieldinduced drift and deformation of spiral waves in an excitable medium, Phys. Rev. Lett. 68, 248 (1992).

[63] F. Fenton, S. Evans, and H. Hastings, Memory in an excitable medium: a mechanism for spiral wave breakup in the low-excitability limit, Physical review letters $\mathbf{8 3}$, 3964 (1999).

[64] C. Zemlin and A. Panfilov, Spiral waves in excitable media with negative restitution, Physical Review E. 63, 041912 (2001).

[65] I. Banville and R. Gray, Effect of action potential duration and conduction velocity restitution and their spatial dispersion on alternans and the stability of arrhythmias, Journal of cardiovascular electrophysiology 13, 1141 (2002).

[66] M. Courtemanche, R. J. Ramirez, and S. Nattel, Ionic mechanisms underlying human atrial action potential properties: insights from a mathematical model, Ameri- can Journal of Physiology-Heart and Circulatory Physiology 275, H301 (1998), pMID: 29586616.

[67] C. Tobón, C. A. Ruiz-Villa, E. Heidenreich, L. Romero, F. Hornero, and J. Saiz, A three-dimensional human atrial model with fiber orientation. electrograms and arrhythmic activation patterns relationship, PLOS ONE 8, 1 (2013).

[68] M. Courtemanche, R. J. Ramirez, and S. Nattel, Ionic targets for drug therapy and atrial fibrillationinduced electrical remodeling: insights from a mathematical model, Cardiovascular Research 42, 477 (1999), http://oup.prod.sis.lan/cardiovascres/articlepdf $/ 42 / 2 / 477 / 781318 / 42-2-477$.pdf.

[69] H. Zhang, C. J. Garratt, J. Zhu, and A. V. Holden, Role of up-regulation of IK1 in action potential shortening associated with atrial fibrillation in humans, Cardiovascular Research 66, 493 (2005), http://oup.prod.sis.lan/cardiovascres/articlepdf/66/3/493/953925/66-3-493.pdf.

[70] S. V. Pandit, O. Berenfeld, J. M. Anumonwo, R. M. Zaritski, J. Kneller, S. Nattel, and J. Jalife, Ionic determinants of functional reentry in a 2-d model of human atrial cells during simulated chronic atrial fibrillation, Biophysical Journal 88, 3806 (2005).

[71] L. J. Wang and E. A. Sobie, Mathematical model of the neonatal mouse ventricular action potential, American Journal of Physiology-Heart and Circulatory Physiology 294, H2565 (2008).

[72] J. C. Williams, J. Xu, Z. Lu, A. Klimas, X. Chen, C. M. Ambrosi, I. S. Cohen, and E. Entcheva, Computational optogenetics: Empirically-derived voltage- and light-sensitive channelrhodopsin-2 model, PLOS Computational Biology 9, 1 (2013).

[73] P. M. Boyle, J. C. Williams, C. M. Ambrosi, E. Entcheva, and N. A. Trayanova, A comprehensive multiscale framework for simulating optogenetics in the heart, Nature Communications 4, 2370 (2013). 



\section{Chapter 7}

\section{Discussion and Conclusion}

At the beginning of the thesis, I emphasized that I used optogenetics as a tool to study mechanisms of spiral wave initiation and control in order to deepen our understanding of arrhythmia dynamics. I investigated the control of spiral wave dynamics using light stimuli from sub to supra-threshold intensity. In the following discussion, I will briefly summarise the results and compare them with related studies reported in the literature.

\section{Discussion}

To study the underlying mechanisms of cardiac arrhythmias, genetically modified mice are the most popular and common experimental models used at the molecular and organ level. Ongoing experiments in the field of optogenetics are still mainly performed on the mouse heart $[22,23,99]$. Nevertheless, numerous simulations are performed on cardiomyocyte models of large species such as humans and dogs, with only few studies on rat models $[11,21,123,124]$. In the absence of simulation studies that come close to current experimental models, I mainly used a mathematical model of the mouse ventricular heart in this work.

In the third chapter, the concept of conventional defibrillation was investigated using optogenetics by applying a single global light pulse to terminate a spiral wave in 2D. The question was, how comparable is the mechanism of arrhythmia control with a single global optical pulse, to the conventional method of single-shock defibrillation? The numerical and experimental results showed a similar dose-response curve for both optical and electrical stimulation. An increase in stimulation intensity and pulse duration led to an increase in arrhythmia termination efficiency. However, different mechanisms were at play at different light intensities (LIs). Numerically, I showed that in the case of low LI a transient termination time existed due to the progressive annihilation of the spiral wave starting from the excitable core. In contrast, high LIs excited the entire domain at once, resulting in immediate arrhythmia termination comparable to the traditional defibrillation method. My work was focused mainly on single pulse illumination with light of supra-threshold intensity. 
In chapters 4 and 5, the focus was mainly on exploring arrhythmia control in the sub-threshold LI régime. Functional heterogeneities in cardiac tissue influence the dynamics of a spiral wave. Such heterogeneities can lead either to a variation of the ionic properties of the heart tissue or to an effect of the electrotonic interactions between cardiomyocytes [197]. In chapter 4, I applied three different illumination patterns in a 2D domain with an existing spiral wave: 1) a linear gradient illumination over the entire domain, 2) a one-step illumination in half of the domain, 3) and a multi-step adjusted illumination. Each pattern induced an inhomogeneity across the domain that affects the dynamics of the spiral wave when its tip was exposed to the illuminated area. These patterns modulated the spatial excitability of the system. In all cases, the stationary spiral wave, which naturally rotates with a circular trajectory, started to drift. Applying a linear gradient illumination pattern globally caused the spiral wave to drift regardless of the initial position of the spiral wave tip. This was due to the global inhomogeneity throughout the domain. With local illumination patterns, however, the spiral wave only drifted if its tip was near the boundary between the illuminated and unilluminated area. Applying half-domain illumination to the domain near the tip of the spiral wave resulted in an initially faster drift compared to the linear gradient pattern. This was due to a steeper voltage gradient at the border zone of half-domain illumination. Then, the drift velocity decreased exponentially due to the vanishing gradient outside that zone. This slowed down the drift and caused the spiral wave to rotate with the natural circular trajectory in a homogeneous area. To have a continuous drift the multiple-steps illumination pattern was used where the spiral wave was dragged toward the inexcitable border by decreasing the size of the illuminated region. It was shown that termination efficiency of the multi-step adjusted illumination pattern was higher than the linear gradient illumination pattern.

With all illumination patterns, the spiral wave drifts towards the region with higher LI, where the spiral wave rotates with a longer period. This observation leads to the hypothesis of a new underlying mechanism of defibrillation in optogenetics. The supra-threshold illumination creates a gradient through the heart tissue from the epicardium to the endocardium due to the decrease in light penetration. In this state, the rotating spiral waves experience the inhomogeneity and drift along the illumination gradient to the epicardium, the area with the higher light intensity. In a study by P. Sasse et al. [214], the authors investigated different possible mechanisms of optogenetic defibrillation compared to the conventional methods. The theory of critical mass [215] can provide a possible explanation for the mechanism of optogenetic defibrillation. In this model, a certain amount of cardiac tissue is required to maintain fibrillation. If the amount of excited tissue is greater than the critical mass, successful defibrillation occurs. This shows that depolarisation of all tissue is not necessary for defibrillation. This model can easily be extended to explain a possible mechanism of the optogenetic defibrillation method with global and structured illumination. In the former, epicardial illumination depolarizes a critical mass of light-responsive cardiac tissue globally and fills the excitable gap (the excitable region between two successive wavefronts of the 
spiral wave). Fig.7.1-A represents a rotating spiral wave in the cardiac tissue where it is terminated by global illumination as it is shown in Fig.7.1-B. In the latter, however, the structured illumination pattern during multiple pulses fills the gap by inducing a spiral wave at the vulnerable window (the functional heterogeneity at the tail of an excitation wave provides a necessary condition for the induction of a spiral wave) [216, 217], as shown in Fig.7.1-C.

A

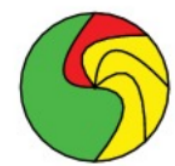

B

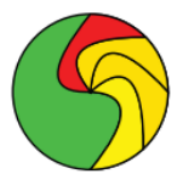

$\mathrm{C}$

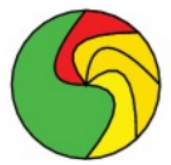

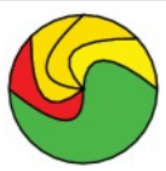
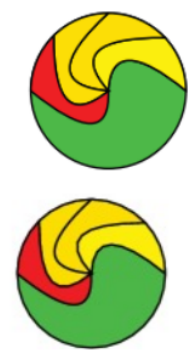
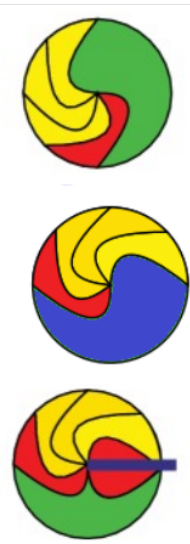
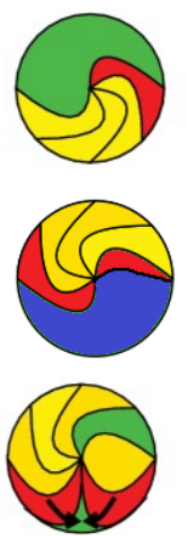
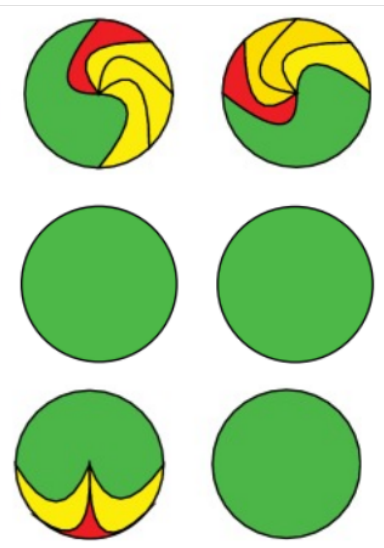

Figure 7.1 - Schematic representation of the mechanism for terminating the arrhythmia when supra-threshold stimulation is applied. A) Propagation of a single spiral wave in the heart tissue. The green, red, and yellow areas illustrate the excitable gap, the wave front, and the wave back. B) Global illumination leads to the filling of the excitable gap through its depolarization. C) Structured illumination induces a spiral wave at the tail of the wave. This leads to a extinction of the two waves by their collision. Taken from [214], an open access article.

Furthermore, Dillon et al. [218] provided optical mapping data showing the effect of electrical stimulation on the increase in action potential duration (APD) with simultaneous extension of the wavelength of the travelling wave. With increase of APD, the excitable region for the spiral wave to propagate is reduced. This results in termination of the spiral waves $[43,44]$. Fig.7.2 shows experimental and numerical studies which demonstrate the effect of the sub-threshold illumination on prolongation of APD. Therefore, the excitable gap can be occupied by the rotating waves of prolonged wavelength by optical stimulation.

In a study by R. Q. Uribe et al. [23], my colleagues controlled arrhythmia activity in the genetically modified mouse heart with a single supra-threshold light pulse applied globally to the heart in different PL and LI. Based on the reported monophasic action potential (MAP) signals, some successful terminations were followed by non-immediate termination, which requires a certain transition period. 

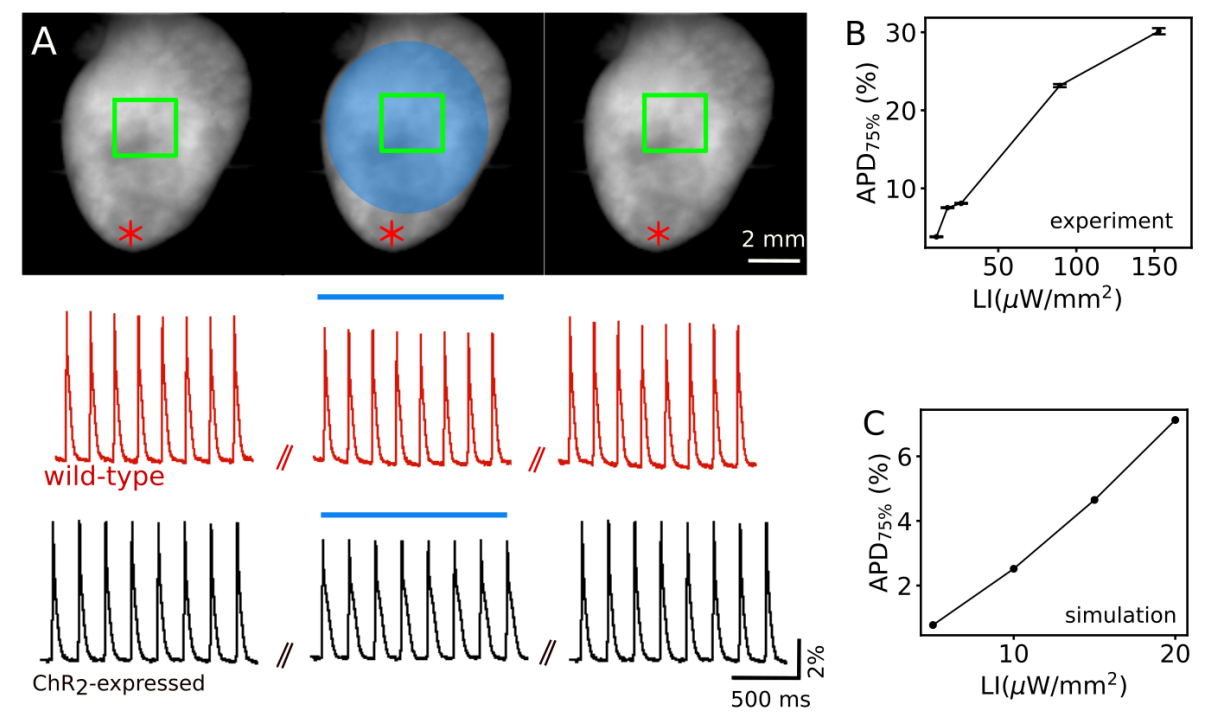

Figure 7.2 - Effect of illumination on the action potential duration. A) Frames of an intact optogenetic mouse heart before (left), during (middle), and after (right) illumination. The corresponding voltage time series are presented in red (black) trace for a wild-type (ChR2-expressed) mouse heart, recorded within the green box. The red star indicates the location of the electrical stimulation. B) APD variation versus different light intensities in the experiment $(N=3$, percentage of termination rate reported as mean $\pm S E M)$. C) $A P D$ variation versus different $L I s$ in a $2 D$ domain of cardiac tissue of mouse. The experimental studies have been done by Valentina Biasci.

Our numerical studies suggest two possible explanations for this observation: 1) A spiral wave rotating below the surface needs a minimal time to drift with the gradient induced by the global illumination (as it is discussed in chapter 3) to the heart surface, where the excitable gap is filled with the depolarized regions. 2) In the case of a spiral wave propagating near the surface, a minimum time is required, either for the spreading of an excitation wave to begin from the excitable region of the spiral wave's core, or for the prolongation of the waveback (as we showed in Fig.7.2), which leads to the filling of the excitable gap. In each of these cases, a longer transient time may occur when a lower LI value is applied due to the slow activation of ChR2.

Temporal modulation of the excitability of the domain is also one possible way of controlling the dynamics of a spiral wave $[92,94,189,210]$. In chapter 5 , I showed how to control the dynamics of a spiral wave by applying a global periodic sub-threshold illumination with different frequencies $\left(f_{p}\right)$ depending on the frequency of the spiral wave $\left(f_{s}\right)$. Under the condition of $f_{p}<f_{s}$, the trajectory of the tip of the spiral wave changes from a circular pattern to a hypocycloid pattern with pedals pointing outwards. If $f_{p}>f_{s}$, the circular trajectory of the tip of the spiral wave changes to an epicycloid pattern with the pedals pointing inwards. I also show how periodic illumination with an open loop pacing, fulfilling the condition $f_{p}=f_{s}$, induces a resonant drift of the spiral wave. This leads to a resonant drift induced collision with the boundary in favor 
of termination. To induce the resonant drift at different light intensities (LI), $f_{p}$ must be adjusted according to the respective LI. To rectify this, a feedback method was used. This method fulfills the condition of $f_{p}=f_{s}$ independently of the LI by recording the $f_{s}$ using a measuring electrode. The application of this method results in the spiral wave being driven in any desired direction.

To show an application of this method, I investigated the termination of the spiral wave from sub-threshold to supra-threshold illumination along the dose-response curve diagram. Along this curve, the underlying mechanism of termination for sub-threshold illumination is the feedback-induced resonant drift, whereas for supra-threshold illumination it is explained by the theory of critical mass. A comparison of the termination rate of feedback pacing with non-feedback pacing showed a higher termination efficiency at sub-threshold illumination for the case of feedback pacing. The experimental studies showed consistent findings with these numerical studies. They both demonstrated that, in the case of feedback pacing, the energy required for spiral wave termination is comparable with increasing intensity. However, the termination time at low LIs is longer than at high LIs, due to the different underlying termination mechanism. Numerically, it is shown that at sub-threshold illumination, the core of the spiral wave is slowly pushed towards the inexcitable boundary, whereas, at supra-threshold illumination, an abrupt excitation of the entire tissue prevents the spiral wave to propagate further which may results in termination. Fig.7.3 shows an overview of the underlying mechanism of arrhythmia control used in this study. In the absence of stimulation light, the spiral wave rotates stationary around an excitable core region. The centre of this core is indicated by a white dashed line in a space-time plot of membrane voltage shown in Fig.7.3-A. At a single global optical pulse with low LI and long illumination time, this excitable region is activated and during the core dissolution process, the spiral wave is progressively terminated. Fig.7.3-D shows the space-time plot of the membrane voltage during the core dissolution of two LIs, 25 and $30 \mu \mathrm{W} / \mathrm{mm}^{2}$ during $500 \mathrm{~ms}$ of a continuous illumination. It illustrates that in both cases there is a transient termination time that is longer for the lower LI than for the higher LI. In contrast, at high LI such as $1000 \mu \mathrm{W} / \mathrm{mm}^{2}$, as shown in Fig.7.3-D, there is an abrupt termination of the spiral wave with a short temrination time, less than $20 \mathrm{~ms}$. Feedback pacing is another method of arrhythmia control investigated in this study. At very low LIs, the spiral wave is pushed towards the boundary by the feedback-induced resonance drift. At high LIs, however, the spiral wave is terminated immediately, as described previously. Fig.7.3-C shows a feedback resonant drift of the spiral wave when a sequence of global pulses is applied to the spiral wave. This causes the spiral wave to drift towards the inexcitable boundary where it annihilates by collision.

As mentioned earlier, the most effective method of eliminating the abnormal electrical activity in heart is to reset its function by complete electrical synchronization. 


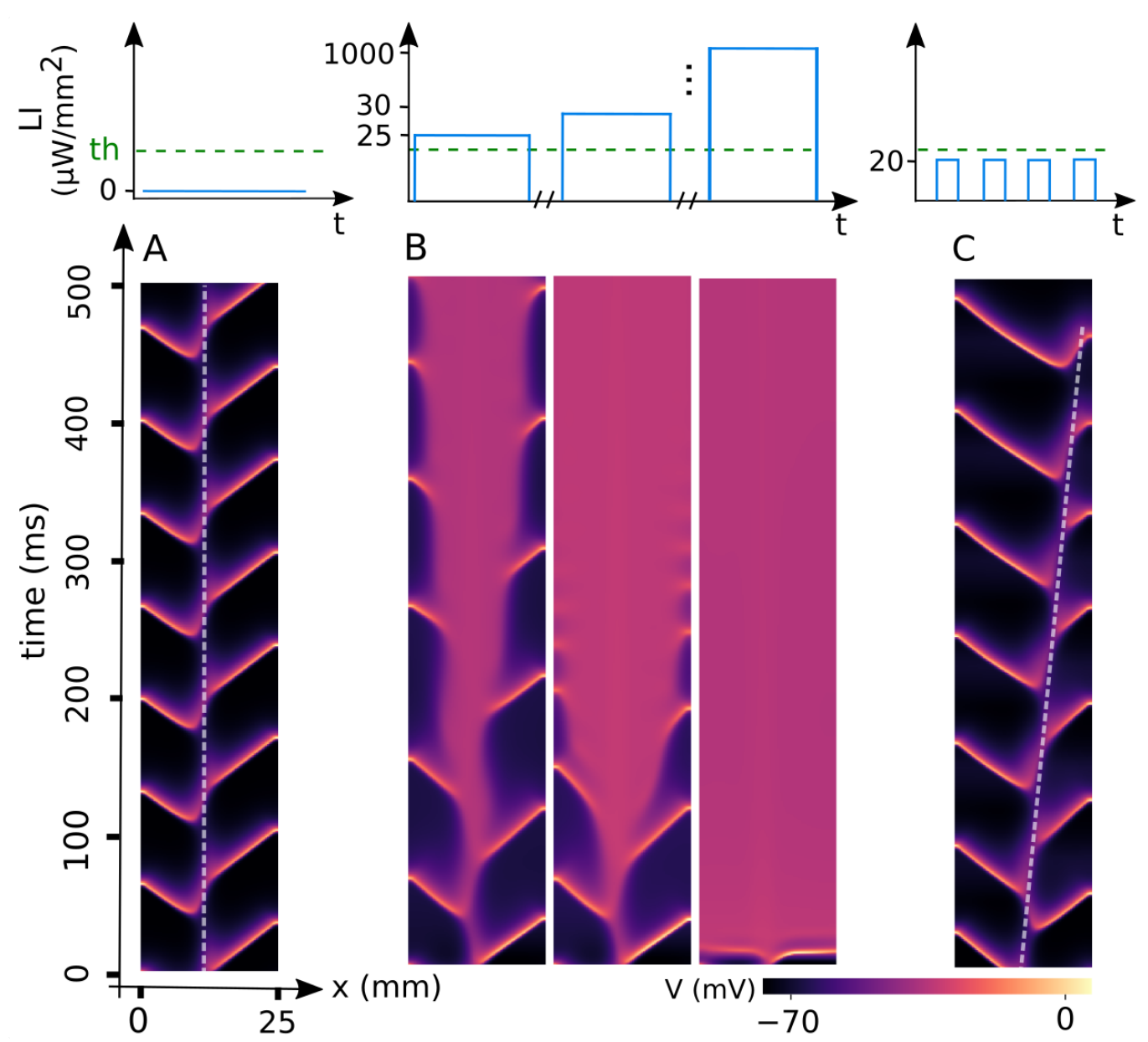

Figure 7.3 - Overview of the underlying mechanisms of arrhythmia termination. A) a space-time plot of the membrane voltage along the $\mathrm{x}$-axis and $\mathrm{y}=12.5 \mathrm{~mm}$ of a $2 \mathrm{D}$ domain $(25 \mathrm{~mm} \times 25 \mathrm{~mm})$ with a rotating spiral wave with a circular core located at the middle of the domain. The dashed line shows the membrane voltage activity of the middle of the core. B) a space-time plot of the membrane voltage during $500 \mathrm{~ms}$ of a single supra-threshold illumination with $\mathrm{LI}$ of 25,30 , and $1000 \mu \mathrm{W} / \mathrm{mm}^{2}$. C) a space-time plot of the membrane voltage during feedback pacing with the PL of $20 \mathrm{~ms}$. The dashed line indicates the drift of the spiral wave towards the inexcitable boundary where it annihilates by collision.

This is achieved by applying strong, globally resetting electric shocks to the heart using defibrillators or cardioverters. However, such high-voltage shocks have significant negative side effects, for example tissue damage, pain and trauma. Therefore, much research is being done to develop low-energy defibrillation techniques [6, 7]. Fig.7.4-A shows the study of an experimental investigation in ex vivo Langendorff-perfused intact pig heart in temperature-controlled perfusion bath. The dose-response curve of electrical defibrillation rate is shown in (B) for two different methods : 1) a single electric shock, indicated by blue symbols and 2) low-energy defibrillation, indicated by orange symbols. For the single electric shock, a biphasic pulse with a duration of $7 \mathrm{~ms}$ was applied via 
mesh electrodes (distance $9.5 \mathrm{~cm}$ ). In the case of low-energy defibrillation, 10 electrical pulses with a frequency of $50 \mathrm{~Hz}$ was applied to the cardiac tissue. The shift of the dose-response curve to the left for the case of the low-energy defibrillation method compared to the single electric shock results in significant reduction in the energy required for defibrillation. Fig.7.4-C shows a Langendorff-perfused intact optogenetic mouse heart in a temperature-controlled perfusion bath. The dose-response curve shows the termination rate as a function of different LIs for three different methods: 1) single global optical pulse (it is shown in light blue for $\mathrm{PL}=10 \mathrm{~ms}$ and dark blue for $\mathrm{PL}=100 \mathrm{~ms}$ ), 2) non-feedback pacing (light orange) and 3) feedback pacing (dark orange). This graph shows a shift in termination rate towards the lower LIs when the termination method changes from a single optical pulse to feedback pacing. The dose-response curve shows 50\% termination rate for the PLs of $10 \mathrm{~ms}$ and $100 \mathrm{~ms}$ around LIs of $200 \mu \mathrm{W} / \mathrm{mm}^{2}$.
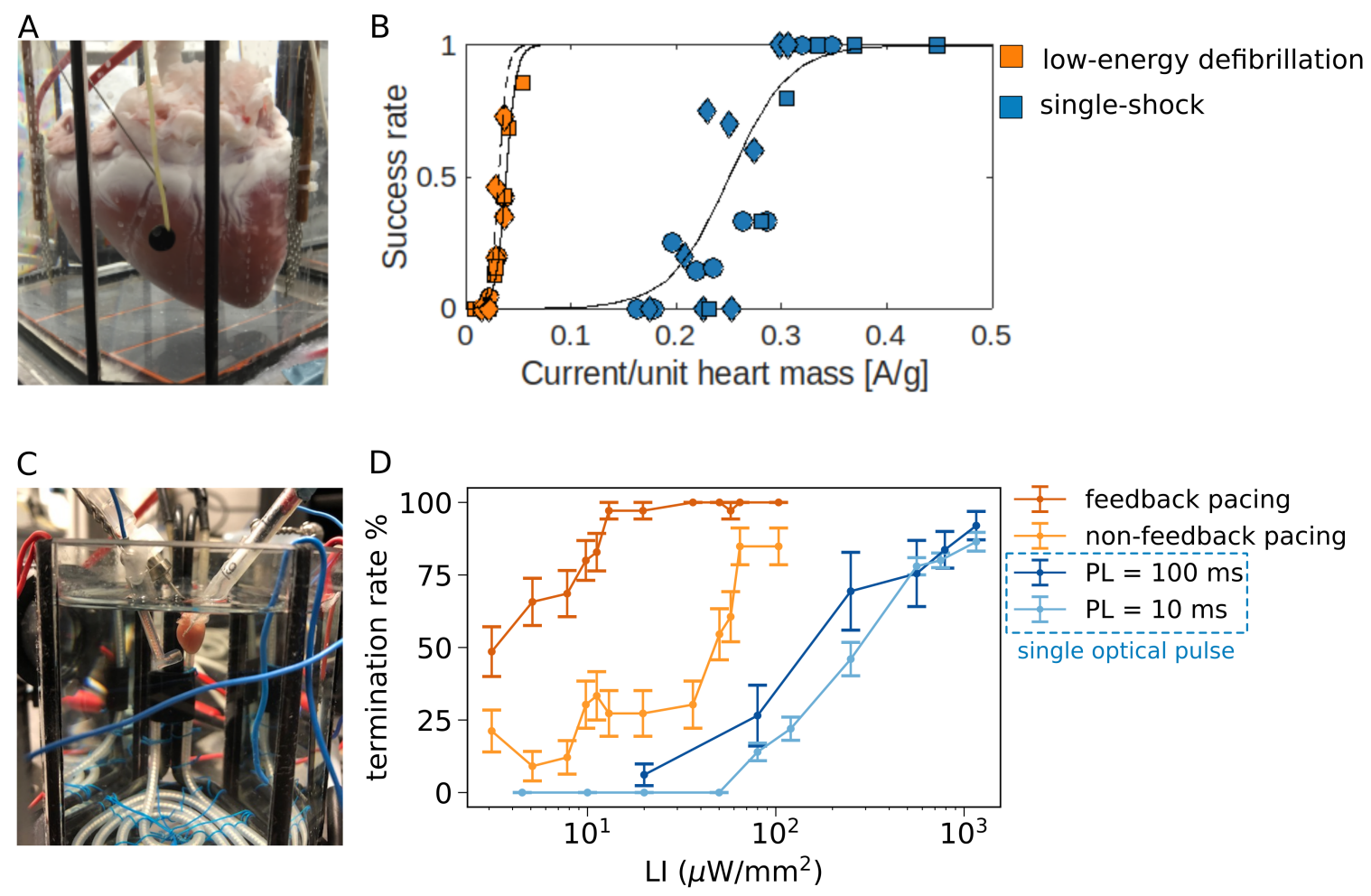

Figure 7.4 - Electrical and optical control of arrhythmia of ex vivo study. A) Langendorffperfused intact pig heart in a temperature-controlled perfusion bath. B) Dose-response diagram for a single biphasic shock ( $P L=7 \mathrm{~ms}$, blue symbols) in $N=3$ pigs. Low-energy defibrillation with 10 biphasic pulses in overdrive (orange symbols) in $N=3$. Defibrillation current is normalised to cardiac mass. C) Langendorff-perfused intact optogenetic mouse heart in temperature-controlled perfusion bath. D) Dose-response diagram for a single optical pulse ( $P L=10 \mathrm{~ms}$ and $100 \mathrm{~ms}$, shown in blue) in $N=5$ mice. A sequence of pulses with PL of $20 \mathrm{~ms}$ during feedback and non-feedback pacing (overdrive pacing with frequency of $30 \mathrm{~Hz}$ ), shown in dark and light orange, respectively, in $N=5$ mice. 
For non-feedback stimulation, applying a sequence of global optical pulses at a frequency of $30 \mathrm{~Hz}$, the termination rate at $50 \%$ is reduced to $60 \mu \mathrm{W} / \mathrm{mm}^{2}$. With feedback stimulation, the termination rate at $50 \%$ is reduced much more to $3 \mu \mathrm{W} / \mathrm{mm}^{2}$. The numerical studies in Chapters 5 and 6 suggest that the high termination rate when using feedback pacing may be due to the termination mechanism of resonance drift at low LIs.

So far in chapters 3, 4, and 5 I have shown the applicability of optogenetics technique as a tool to control a spiral wave in favor of its termination in cardiac tissue. In chapter 6 , it is shown how at a very narrow range of light intensities at the sub-threshold region causes a wave break, which puts the system into a more chaotic and life-threatening state. In this chapter, the effect of sub-threshold illumination in different phases of action potential (AP) was investigated. The results indicate the phase-dependent recovery that can lead to the different conduction velocities (CV) along a propagating wave in an extended range. Global illumination with long sub-threshold illumination causes the elongation of the waveback, which leads to a change in the morphology of the spatial profile of the wave. As a result, a conduction block is created by the waveback of the preceding wave for the following wave. When the light is turned off, the wave profile changes to a pinched appearance where the distance between the cells at the tail of the wave and the wavefront is reduced. This results in the appearance of a vulnerable window that can be re-excited. So the application of periodic sub-threshold illumination leads to the occurrence of wave break, which causes the waves to re-excite and rotate within the vulnerable window. In this chapter two different ionic models, one representing the cardiac tissue of human atrium and the other a neonatal mouse model, were used to show that this wave break phenomena at homogenuous 2D domain.

\section{Conclusion}

In this thesis, the potential of optogenetics as a valuable tool to deepen our understanding of arrhythmia dynamics and its control was explored. By using 2D simulation which describes spiral wave dynamics in cardiac tissue, this study provides a mechanistic insight into optogenetic defibrillation. The numerical observations provide explanations for the non-spontaneous termination observed in experimental studies. This work demonstrated the use of functional heterogeneity and periodic pacing to optically induce spiral wave drift. Which can direct the spiral wave towards a collision with the boundary in favor of termination. The termination efficiency of cardiac arrhythmias was investigated experimentally and numerically using a single optical pulse or a train of periodic pulses. The results suggest the potential of feedback-induced resonant pacing as a low-energy defibrillation method. In addition, optogenetics was used to provide a clear example of a mechanism of wave break of a rotating spiral wave in a homogeneous heart tissue.

This work successfully demonstrated multiple approaches to control the dynamics of a single spiral wave in a 2D domain using optogenetics. Future works should aim for controlling the dynamics of a chaotic system with multiple spiral waves. While the $e x$ 
vivo experiments on the mouse heart were very insightful in agreement with the current numerical simulations, currently, the research on the development of transgenic large animal models is ongoing. Should they become available, it would be interesting to investigate how the mechanisms found in our study on mouse hearts translate to larger mammalian hearts, that can show much more complex spatial-temporal dynamics during arrhythmias. 



\section{References}

[1] M. G. R. "Some observations on electrograms of the frog's heart". In: Proc Camb Phil Soc 16 (1912), pp. 615-620.

[2] M. G. R. "On dynamic equilibrium in the heart". In: Proc Camb Phil Soc 46.4-5 (1913), pp. 349-383. DOI: 10.1113/jphysiol.1913.sp001596.

[3] D. J. Dosdall, V. G. Fast, and R. E. Ideker. "Mechanisms of defibrillation." In: Annual review of biomedical engineering 12 (2010), pp. 215-228. DOI: 10.1146/ annurev-bioeng-070909-105305.

[4] S. C. VLAY, L. C. OLSON, G. L. FRICCHIONE, and R. FRIEDMAN. "Anxiety and anger in patients with ventricular tachyarrhythmias. Responses after automatic internal cardioverter defibrillator implantation". In: Pacing and Clinical Electrophysiology 12.2 (1989), pp. 366-373.

[5] B. Lüderitz, W. Jung, A. Deister, and M. Manz. "Patient acceptance of implantable cardioverter defibrillator devices: changing attitudes". In: American heart journal 127.4 (1994), pp. 1179-1184.

[6] S. Luther et al. "Low-energy control of electrical turbulence in the heart". In: Nature 475 (7355 July 2011), pp. 235-239. DOI: 10.1038/nature10216.

[7] F. Fenton, S. Luther, E. Cherry, N. Otani, V. Krinsky, A. Pumir, E. Bodenschatz, and R. J. Gilmour. "Termination of atrial fibrillation using pulsed low-energy far-field stimulation". In: Circulation 120 (6 June 2009), pp. 467-476. DOI: 10.1161/CIRCULATIONAHA.108.825091. 
[8] C. M. Ambrosi, C. M. Ripplinger, I. R. Efimov, and V. V. edorov. "Termination of sustained atrial flutter and fibrillation using low-voltage multiple-shock therapy". In: Heart Rhythm 8 (1 Jan. 2011). DOI: 10.1016/j.hrthm.2010.10.018.

[9] W. Li, C. M. Ripplinger, Q. Lou, and I. R. Efimov. "Multiple monophasic shocks improve electrotherapy of ventricular tachycardia in a rabbit model of chronic infarction". In: Heart Rhythm 6 (7 July 2009). DOI: 10.1016/j.hrthm.2009.03.015.

[10] R. Burton, A. Klimas, C. Ambrosi, J. Tomek, A. Corbett, E. Entcheva, and G. Bub. "Optical control of excitation waves in cardiac tissue". In: Nature Photon 9 (12 Oct. 2015), pp. 813-816. DOI: 10.1038/nphoton.2015.196.

[11] R. Majumder, I. Feola, A. S. Teplenin, A. A. de Vries, A. V. Panfilov, and D. A. Pijnappels. "Optogenetics enables real-time spatiotemporal control over spiral wave dynamics in an excitable cardiac system". In: eLife 7 (Sept. 2018). Ed. by L. Glass and A. K. Chakraborty, e41076. Dor: 10.7554/eLife.41076.

[12] E. Callaway. "Shining new light on neural circuits". In: Science 314 (2006), pp. $1674-1674$.

[13] A. Destexhe and D. Paré. "Impact of network activity on the integrative properties of neocortical pyramidal neurons in vivo". In: Journal of neurophysiology 81.4 (1999), pp. 1531-1547.

[14] K. Deisseroth, G. Feng, A. Majewska, G. Miesenböck, A. Ting, and M. Schnitzer. "Next-generation optical technologies for illuminating genetically targeted brain circuits". In: J Neurosci 26 (41 2006), pp. 10380-10386. DOI: 10.1523/JNEUROSCI.3863-06.2006.

[15] T. Ishizuka, M. Kakuda, R. Araki, and H. Yawo. "Kinetic evaluation of photosensitivity in genetically engineered neurons expressing green algae light-gated channels". In: Neurosci Res 54 (2 2005), pp. 85-94. DOI: 10.1016/j.neures.2005.10.009.

[16] M. S. Sakar, D. Neal, T. Boudou, M. A. Borochin, Y. Li, R. Weiss, R. D. Kamm, C. S. Chen, and H. H. Asada. "Formation and optogenetic control of engineered 3D skeletal muscle bioactuators". In: Lab on a Chip 12.23 (2012), pp. 4976-4985. 
[17] T. van Bremen, T. Send, P. Sasse, and T. Bruegmann. "Spot light on skeletal muscles: optogenetic stimulation to understand and restore skeletal muscle function". In: Journal of muscle research and cell motility 38.3-4 (2017), pp. 331337.

[18] T. Bruegmann, T. Van Bremen, C. C. Vogt, T. Send, B. K. Fleischmann, and P. Sasse. "Optogenetic control of contractile function in skeletal muscle". In: Nature communications 6.1 (2015), pp. 1-8.

[19] E. Entcheva. "Cardiac optogenetics". In: American Journal of Physiology-Heart and Circulatory Physiology 304.9 (2013), H1179-H1191.

[20] E. Entcheva and G. Bub. "All-optical control of cardiac excitation: combined high-resolution optogenetic actuation and optical mapping". In: The Journal of Physiology 594.9 (2016), pp. 2503-2510. DOI: 10.1113/JP271559. eprint: https://physoc.onlinelibrary.wiley.com/doi/pdf/10.1113/JP271559.

[21] T. Bruegmann, P. M. Boyle, C. C. Vogt, T. V. Karathanos, H. J. Arevalo, B. K. Fleischmann, N. A. Trayanova, and P. Sasse. "Optogenetic defibrillation terminates ventricular arrhythmia in mouse hearts and human simulations". In: The Journal of Clinical Investigation 126.10 (Oct. 2016), pp. 3894-3904. DOI: 10.1172/JCI88950.

[22] C. Crocini, C. Ferrantini, F. S. Pavone, and L. Sacconi. "Optogenetics gets to the heart: A guiding light beyond defibrillation". In: Progress in Biophysics and Molecular Biology 130 (2017). Cardiac Mechanics and Electrics: it takes two to tango, pp. 132-139. DOI: https://doi.org/10.1016/j.pbiomolbio.2017.05.002.

[23] R. A. Quiñonez Uribe, S. Luther, L. Diaz-Maue, and C. Richter. "EnergyReduced Arrhythmia Termination Using Global Photostimulation in Optogenetic Murine Hearts". In: Frontiers in Physiology 9 (2018), p. 1651. DOI: 10.3389/ fphys.2018.01651.

[24] M. Gavaghan. "Cardiac Anatomy and Physiology: A Review". In: AORN Journal 67.4 (1998), pp. 800-822. DOI: https://doi.org/10.1016/S0001-2092(06)62644-6. 
[25] M. E. Silverman, D. Grove, and C. B. Upshaw Jr. "Why does the heart beat? The discovery of the electrical system of the heart". In: Circulation 113.23 (2006), pp. 2775-2781.

[26] D. L. Wolfe. Electrode system for acquiring electrical signals from the heart. US Patent 4,120,294. Oct. 1978.

[27] L. M. Biga, S. Dawson, A. Harwell, R. Hopkins, J. Kaufmann, M. LeMaster, P. Matern, K. Morrison-Graham, D. Quick, and J. Runyeon. Anatomy Physiology. Oregon State University., 2018.

[28] U. P. S. T. Force. "Screening for Cardiovascular Disease Risk With Electrocardiography: US Preventive Services Task Force Recommendation Statement". In: JAMA 319.22 (June 2018), pp. 2308-2314. DOI: 10.1001/jama.2018.6848. eprint: https://jamanetwork.com/journals/jama/articlepdf/2684613/jama\_curry $\backslash$ _2018\_us\_180010.pdf.

[29] G. Shelton and D. Jones. "The physiology of the alligator heart: the cardiac cycle". In: Journal of experimental biology 158.1 (1991), pp. 539-564.

[30] F. K. Marcondes, M. J. Moura, A. Sanches, R. Costa, P. O. de Lima, F. C. Groppo, M. E. Amaral, P. Zeni, K. C. Gaviao, and L. H. Montrezor. "A puzzle used to teach the cardiac cycle". In: Advances in Physiology Education 39.1 (2015), pp. 27-31.

[31] S. C. Health. Electrical System of the Heart. Accessed: 2020.

[32] K. R and S. K. Chemical Waves and Patterns. Springer, Dordrecht, 1995.

[33] J. M. Greenberg and S. P. Hastings. "Spatial Patterns for Discrete Models of Diffusion in Excitable Media". In: SIAM Journal on Applied Mathematics 34.3 (1978), pp. 515-523. DOI: 10.1137/0134040. eprint: https://doi.org/10.1137/ 0134040 . 
[34] A. T. Winfree. "Spiral Waves of Chemical Activity". In: Science 175.4022 (1972), pp. 634-636. DOI: 10.1126/science.175.4022.634. eprint: https://science. sciencemag.org/content/175/4022/634.full.pdf.

[35] K. VI. "Mathematical models of cardiac arrhythmias (spiral waves)." In: Pharmacol Ther B 3.4 (1978), pp. 539-555. DOI: 10.1016/s0306-039x(78)90020-x.

[36] R. Majumder, R. Pandit, and A. V. Panfilov. "Turbulent electrical activity at sharp-edged inexcitable obstacles in a model for human cardiac tissue". In: American Journal of Physiology-Heart and Circulatory Physiology 307.7 (2014). PMID: 25108011, H1024-H1035. DOI: 10.1152/ajpheart.00593.2013. eprint: https://doi.org/10.1152/ajpheart.00593.2013.

[37] Z. Qu and F. Xie. "Origins of Spiral Wave Meander and Breakup in a TwoDimensional". In: Annals of Biomedical Engineering 28.7 (2000), pp. 775-771. DOI: $10.1114 / 1.1289474$.

[38] G. Bub, A. Shrier, and L. Glass. "Spiral wave generation in heterogeneous excitable media”. In: Physical review letters 88.5 (2002), p. 058101.

[39] M. G. R. "On dynamic equilibrium in the heart". In: TRANS. R.S.D 8 (1914), pp. $43-53$.

[40] W. E. Garrey. "The nature of fibrillary contraction of the heart.-Its relation to tissue mass and form". In: American Journal of Physiology-Legacy Content 33.3 (1914), pp. 397-414. DOI: 10.1152 / ajplegacy. 1914.33 .3 .397. eprint: https://doi.org/10.1152/ajplegacy.1914.33.3.397.

[41] A. Karma. "Electrical alternans and spiral wave breakup in cardiac tissue". In: Chaos: An Interdisciplinary Journal of Nonlinear Science 4.3 (1994), pp. 461472. DOI: 10.1063/1.166024. eprint: https://doi.org/10.1063/1.166024.

[42] M. Gerhardt, S. Heike, and T. John J. "A cellular automation model of excitable media including curvature and dispersion". In: Science 247.4950 (1990), pp. 15631566. DOI: $10.1126 /$ science.2321017. 
[43] T. Lilienkamp, J. Christoph, and U. Parlitz. "Features of chaotic transients in excitable media governed by spiral and scroll waves". In: Physical Review Letters 119.5 (2017), p. 054101.

[44] T. Lilienkamp and U. Parlitz. "Terminal transient phase of chaotic transients". In: Physical review letters 120.9 (2018), p. 094101.

[45] M. E. Josephson, L. N. Horowitz, A. Farshidi, and J. A. Kastor. "Recurrent sustained ventricular tachycardia. 1. Mechanisms." In: Circulation 57.3 (1978), pp. $431-440$.

[46] H. J. Wellens, R. M. Schuilenburg, and D. Durrer. "Electrical stimulation of the heart in patients with ventricular tachycardia". In: Professor Hein JJ Wellens. Springer, 2000, pp. 43-53.

[47] J. M. Mangrum and J. P. DiMarco. "The evaluation and management of bradycardia". In: New England Journal of Medicine 342.10 (2000), pp. 703-709.

[48] D. Short. "The syndrome of alternating bradycardia and tachycardia". In: British heart journal 16.2 (1954), p. 208.

[49] B. Lown and R. L. Verrier. "Neural activity and ventricular fibrillation". In: New England Journal of Medicine 294.21 (1976), pp. 1165-1170.

[50] C. J. Wiggers. "The mechanism and nature of ventricular fibrillation". In: American Heart Journal 20.4 (1940), pp. 399-412.

[51] J. N. Weiss, Z. Qu, P.-S. Chen, S.-F. Lin, H. S. Karagueuzian, H. Hayashi, A. Garfinkel, and A. Karma. "The Dynamics of Cardiac Fibrillation". In: Circulation 112.8 (2005), pp. 1232-1240. DOI: 10.1161/CIRCULATIONAHA.104.529545. eprint: https://www.ahajournals.org/doi/pdf/10.1161/CIRCULATIONAHA. 104.529545 .

[52] J. B. Shea and W. H. Maisel. "Cardioversion". In: Circulation 106.22 (2002). PMID: 25108011, e176-e178. DOI: 10.1161/01.CIR.0000040586.24302.B9. eprint: https://doi.org/10.1161/01.CIR.0000040586.24302.B9. 
[53] M. S. Wathen et al. "Prospective Randomized Multicenter Trial of Empirical Antitachycardia Pacing Versus Shocks for Spontaneous Rapid Ventricular Tachycardia in Patients With Implantable Cardioverter-Defibrillators". In: Circulation 110.17 (2004), pp. 2591-2596. DOI: 10.1161/01.CIR.0000145610.64014.E4.

[54] J. Abboud and J. R. Ehrlich. "Antiarrhythmic drug therapy to avoid implantable cardioverter defibrillator shocks". In: Arrhythmia \& electrophysiology review 5.2 (2016), p. 117.

[55] A. Bollmann, D. Husser, and D. S. Cannom. "Antiarrhythmic drugs in patients with implantable cardioverter-defibrillators". In: American journal of cardiovascular drugs 5.6 (2005), pp. 371-378.

[56] S. Priori, C. Blomström-Lundqvist, A. Mazzanti, N. Blom, M. Borggrefe, J. Camm, P. Elliott, D. Fitzsimons, R. Hatala, G. Hindricks, et al. "ESC Scientific Document Group. 2015 ESC Guidelines for the management of patients with ventricular arrhythmias and the prevention of sudden cardiac death: The Task Force for the Management of Patients with Ventricular Arrhythmias and the Prevention of Sudden Cardiac Death of the European Society of Cardiology (ESC). Endorsed by: Association for European Paediatric and Congenital Cardiology (AEPC)". In: Eur Heart J 36.41 (2015), pp. 2793-867.

[57] W. D. Weaver, M. K. Copass, D. L. Hill, C. Fahrenbruch, A. P. Hallstrom, and L. A. Cobb. "Cardiac arrest treated with a new automatic external defibrillator by out-of-hospital first responders". In: The American journal of cardiology 57.13 (1986), pp. 1017-1021.

[58] N. Jaggarao, R. Grainger, M. Heber, R. Vincent, D. Chamberlain, and A. Aronson. "Use of an automated external defibrillator-pacemaker by ambulance staff". In: The Lancet 320.8289 (1982), pp. 73-75.

[59] A. Diack, W. Welborn, R. Rullman, C. Walter, and M. Wayne. "An automatic cardiac resuscitator for emergency treatment of cardiac arrest." In: Medical instrumentation 13.2 (1979), pp. 78-83. 
[60] H. Kamphuis, J. De Leeuw, R. Derksen, R. Hauer, and J. Winnubst. "Implantable cardioverter defibrillator recipients: quality of life in recipients with and without ICD shock delivery: a prospective study". In: EP Europace 5.4 (2003), pp. 381-389.

[61] G. L. Fricchione, L. C. Olson, and S. C. Vlay. "Psychiatric syndromes in patients with the automatic internal cardioverter defibrillator: anxiety, psychological dependence, abuse, and withdrawal." In: American heart journal 117.6 (1989), p. 1411.

[62] P. R. Kowey. "The calamity of cardioversion of conscious patients." In: The American journal of cardiology 61.13 (1988), p. 1106.

[63] M. S. Wathen et al. "Prospective Randomized Multicenter Trial of Empirical Antitachycardia Pacing Versus Shocks for Spontaneous Rapid Ventricular Tachycardia in Patients With Implantable Cardioverter-Defibrillators". In: Circulation 110.17 (2004), pp. 2591-2596. DOI: 10.1161/01.CIR.0000145610.64014.E4.

[64] N. Bursac and L. Tung. "Acceleration of functional reentry by rapid pacing in anisotropic cardiac monolayers: Formation of multi-wave functional reentries". In: Cardiovascular Research 69.2 (Feb. 2006), pp. 381-390. DOI: 10.1016/j. cardiores.2005.09.014. eprint: https://academic.oup.com/cardiovascres/articlepdf/69/2/381/796304/69-2-381.pdf.

[65] J. M. Davidenko, R. Salomonsz, A. M. Pertsov, W. T. Baxter, and J. Jalife. "Effects of pacing on stationary reentrant activity: theoretical and experimental study". In: Circulation research 77.6 (1995), pp. 1166-1179.

[66] K. Kamjoo, T. Uchida, T. Ikeda, M. C. Fishbein, A. Garfinkel, J. N. Weiss, H. S. Karagueuzian, and P.-S. Chen. "Importance of location and timing of electrical stimuli in terminating sustained functional reentry in isolated swine ventricular tissues: evidence in support of a small reentrant circuit". In: Circulation 96.6 (1997), pp. 2048-2060. 
[67] C. M. Ripplinger, V. I. Krinsky, V. P. Nikolski, and I. R. Efimov. "Mechanisms of unpinning and termination of ventricular tachycardia". In: American Journal of Physiology-Heart and Circulatory Physiology 291.1 (2006), H184-H192.

[68] K. Agladze, M. Kay, V. Krinsky, and N. Sarvazyan. "Interaction between spiral and paced waves in cardiac tissue". In: Am J Physiol Heart Circ Physiol 293 (1 Dec. 2007), H503-H513. DOI: 10.1152/ajpheart.01060.2006.

[69] A. Pumir, S. Sinha, S. Sridhar, M. Argentina, M. Hörning, S. Filippi, C. Cherubini, S. Luther, and V. Krinsky. "Wave-train-induced termination of weakly anchored vortices in excitable media". In: Phys. Rev. E 81 (1 Jan. 2010), p. 010901. DOI: 10.1103/PhysRevE.81.010901.

[70] A. Muler and V. Markin. "Distribution of the electrotonic potential". In: Biofizika 22.2 (1977), pp. 307-312.

[71] D. Geselowitz and W. Miller. "A bidomain model for anisotropic cardiac muscle". In: Ann Biomed Eng 11.3-4 (1983), pp. 191-206. DOI: 10.1007/BF02363286.

[72] N. Sepulveda, B. Roth, and J. J. Wikswo. "Current injection into a twodimensional anisotropic bidomain". In: Biophys J 55.5 (1989), pp. 987-999. DoI: 10.1016/S0006-3495(89)82897-8.

[73] D. L. Janks and B. J. Roth. "The bidomain theory of pacing". In: Cardiac Bioelectric Therapy. Springer, 2009, pp. 63-83.

[74] I. R. Efimov, F. Aguel, Y. Cheng, B. Wollenzier, and N. Trayanova. "Virtual electrode polarization in the far field: implications for external defibrillation". In: American Journal of Physiology-Heart and Circulatory Physiology 279.3 (2000), H1055-H1070.

[75] N. Trayanova, K. Skouibine, and F. Aguel. "The role of cardiac tissue structure in defibrillation". In: Chaos: An Interdisciplinary Journal of Nonlinear Science 8.1 (1998), pp. 221-233. DOI: 10.1063/1.166299. eprint: https://doi.org/10. 1063/1.166299. 
[76] F. Xia, G. Xiang, T. Juan-Mei, P. Jun-Ting, and Z. Hong. "Wave trains induced by circularly polarized electric fields in cardiac tissues." In: Sci Rep 5.13349 (2015), pp. 1-9. DOI: 10.1038/srep13349.

[77] Y. C. Ji, I. Uzelac, N. Otani, S. Luther, R. F. Gilmour, E. M. Cherry, and F. H. Fenton. "Synchronization as a mechanism for low-energy anti-fibrillation pacing”. In: Heart Rhythm 14.8 (2017), pp. 1254-1262. DoI: https://doi.org/10. 1016/j.hrthm.2017.05.021.

[78] G. K. Moe, W. C. Rheinboldt, and J. Abildskov. "A computer model of atrial fibrillation". In: American Heart Journal 67.2 (1964), pp. 200-220. DOI: https: //doi.org/10.1016/0002-8703(64)90371-0.

[79] V. Krinskii. "SPREAD OF EXCITATION IN AN INHOMOGENEOUS MEDIUM (STATE SHVIILAR TO CARDIAC FIBRILLATION)". In: (1966).

[80] K. H. W. J. Ten Tusscher and A. V. Panfilov. "Reentry in heterogeneous cardiac tissue described by the Luo-Rudy ventricular action potential model". In: American Journal of Physiology-Heart and Circulatory Physiology 284.2 (2003). PMID: 12388228, H542-H548. DOI: 10.1152/ajpheart.00608.2002. eprint: https://doi.org/10.1152/ajpheart.00608.2002.

[81] F. L. Burton and S. M. Cobbe. "Dispersion of ventricular repolarization and refractory period". In: Cardiovascular Research 50.1 (Apr. 2001), pp. 10-23. DOI: 10.1016/S0008-6363(01)00197-3. eprint: https:/ / academic.oup.com/ cardiovascres/article-pdf/50/1/10/914666/50-1-10.pdf.

[82] X. Zou, H. Levine, and D. A. Kessler. "Interaction between a drifting spiral and defects". In: Phys. Rev. E 47 (2 Feb. 1993), R800-R803. DoI: 10.1103/ PhysRevE.47.R800.

[83] S. Sridhar, S. Sinha, and A. V. Panfilov. "Anomalous drift of spiral waves in heterogeneous excitable media". In: Phys. Rev. E 82 (5 Nov. 2010), p. 051908. DOI: 10.1103/PhysRevE.82.051908. 
[84] S. Kharche, I. Biktasheva, G. Seemann, H. Zhang, and V. Biktashev. "A Computer Simulation Study of Anatomy Induced Drift of Spiral Waves in the Human Atrium". In: BioMed Research International 2015 (2015). DOI: $10.1155 / 2015 / 731386$.

[85] M. Wellner, C. Zemlin, and A. M. Pertsov. "Frustrated drift of an anchored scroll-wave filament and the geodesic principle". In: Phys. Rev. E 82 (3 Sept. 2010), p. 036122. DOI: 10.1103/PhysRevE.82.036122.

[86] A. M. Pertsov, J. M. Davidenko, R. Salomonsz, W. T. Baxter, and J. Jalife. "Spiral waves of excitation underlie reentrant activity in isolated cardiac muscle." In: Circulation research 72.3 (1993), pp. 631-650.

[87] J. M. Davidenko, A. V. Pertsov, R. Salomonsz, W. Baxter, and J. Jalife. "Stationary and drifting spiral waves of excitation in isolated cardiac muscle". In: Nature 355.6358 (1992), pp. 349-351.

[88] V. Krinsky, E. Hamm, and V. Voignier. "Dense and Sparse Vortices in Excitable Media Drift in Opposite Directions in Electric Field". In: Phys. Rev. Lett. 76 (20 May 1996), pp. 3854-3857. DOI: 10.1103/PhysRevLett.76.3854.

[89] O. Steinbock, J. Schütze, and S. C. Müller. "Electric-field-induced drift and deformation of spiral waves in an excitable medium". In: Phys. Rev. Lett. 68 (2 Jan. 1992), pp. 248-251. DOI: 10.1103/PhysRevLett.68.248.

[90] A. P. Muñuzuri, M. Gómez-Gesteira, V. Pérez-Muñuzuri, V. I. Krinsky, and V. Pérez-Villar. "Mechanism of the electric-field-induced vortex drift in excitable media". In: Phys. Rev. E 48 (5 Nov. 1993), R3232-R3235. DoI: 10.1103/ PhysRevE.48.R3232.

[91] A. Muñuzuri, V. Davydov, V. Pérez-Muñuzuri, M. Gómez-Gesteira, and V. Pérez-Villar. "General properties of the electric-field-induced vortex drift in excitable media". In: Chaos, Solitons Fractals 7.4 (1996). Time Symmetry Breaking in Classical and Quantum Mechanics, pp. 585-595. DoI: https://doi. org/10.1016/0960-0779(95)00061-5. 
[92] K. I. Agladze, V. A. Davydov, and A. S. Mikhařlov. "Observation of a helicalwave resonance in an excitable distributed medium". In: ZhETF Pisma Redaktsiiu 45 (June 1987), p. 601.

[93] A. S. Mikhailov. "Foundations of Synergetics I Distributed Active Systems. Vol. 51 from: Springer Series in Synergetics. Springer Verlag, Berlin, ISBN 3-54052775-3, 1990. 187 Pages, Price: DM 110, -". In: Berichte der Bunsengesellschaft für physikalische Chemie 95.10 (1991), pp. 1308-1309. DOI: 10.1002/bbpc. 19910951040. eprint: https://onlinelibrary.wiley.com/doi/pdf/10.1002/bbpc. 19910951040.

[94] V. Biktashev, I. Biktasheva, and N. Sarvazyan. "Evolution of Spiral and Scroll Waves of Excitation in a Mathematical Model of Ischaemic Border Zone". In: PLoS ONE 6 (9 Sept. 2011). DOI: 10.1371/journal.pone.0024388.

[95] S. Morgan. Low-energy defibrillation using resonant drift pacing. Thesis Ph.D., 2009.

[96] I. Aranson, D. Kessler, and I. Mitkov. "Boundary-induced drift of spirals in excitable media". In: Phys. Rev. E 50 (4 Oct. 1994), R2395-R2398. DoI: 10.1103/PhysRevE.50.R2395.

[97] E. Boyden, F. Zhang, E. Bamberg, G. Nagel, and K. Deisseroth. "Millisecondtimescale, genetically targeted optical control of neural activity". In: Biophys $J$ 8.9 (2005), pp. 1263-1268. DOI: 10.1038/nn1525.

[98] A. Destexhe and D. Paré. "Impact of network activity on the integrative properties of neocortical pyramidal neurons in vivo". In: Journal of neurophysiology 81.4 (1999), pp. 1531-1547.

[99] T. Bruegmann, D. Malan, M. Hesse, T. Beiert, C. J. Fuegemann, B. K. Fleischmann, and P. Sasse. "Optogenetic control of heart muscle in vitro and in vivo". In: Nature Methods 7 (11 Oct. 2010), pp. 897-900. DOI: 10.1038/nmeth.1512. 
[100] A. B. Arrenberg, D. Y. R. Stainier, H. Baier, and J. Huisken. "Optogenetic Control of Cardiac Function". In: Science 330.6006 (2010), pp. 971-974. DOI: 10.1126/science.1195929. eprint: https://science.sciencemag.org/content/330/ 6006/971.full.pdf.

[101] G. Miesenböck. "The optogenetic catechism". In: Science 326.5951 (2009), pp. 395-399.

[102] C. M. Ambrosi and E. Entcheva. "Optogenetic control of cardiomyocytes via viral delivery". In: Methods in molecular biology (Clifton, N.J.) 1181 (2014), pp. 233-258. DOI: 10.1007/978-1-4939-1047-2_19.

[103] C. M. Ambrosi, P. M. Boyle, K. Chen, N. A. Trayanova, and E. Entcheva. "Optogenetics-enabled assessment of viral gene and cell therapy for restoration of cardiac excitability". In: Scientific reports 5 (2015), p. 17350.

[104] Z. Jia, V. Valiunas, Z. Lu, H. Bien, H. Liu, H.-Z. Wang, B. Rosati, P. R. Brink, I. S. Cohen, and E. Entcheva. "Stimulating cardiac muscle by light: cardiac optogenetics by cell delivery". In: Circulation: Arrhythmia and Electrophysiology 4.5 (2011), pp. 753-760.

[105] C. Richter, J. Christoph, S. E. Lehnart, and S. Luther. "Optogenetic light crafting tools for the control of cardiac arrhythmias". In: Optogenetics. Springer, 2016, pp. 293-302.

[106] I. R. Efimov, V. P. Nikolski, and G. Salama. "Optical imaging of the heart". In: Circulation research 95.1 (2004), pp. 21-33.

[107] J. Christoph, M. Chebbok, C. Richter, J. Schröder-Schetelig, P. Bittihn, S. Stein, I. Uzelac, F. Fenton, G. Hasenfuß, R. Gilmour Jr, et al. "Electromechanical vortex filaments during cardiac fibrillation". In: Nature 555.7698 (2018), pp. 667672 .

[108] J. Christoph, J. Schröder-Schetelig, and S. Luther. "Electromechanical optical mapping”. In: Progress in Biophysics and Molecular Biology 130 (2017), pp. 150169. 
[109] N. J. Severs. "The cardiac muscle cell”. In: Bioessays 22.2 (2000), pp. 188-199.

[110] J. Seifter, D. Sloane, and A. Ratner. Concepts in medical physiology. Lippincott Williams \& Wilkins, 2005.

[111] G. von Heijne. "Membrane-protein topology". In: Nature reviews Molecular cell biology 7.12 (2006), pp. 909-918.

[112] G. Karp. Cell and molecular biology: concepts and experiments. John Wiley \& Sons, 2009.

[113] A. M. Katz. "Cardiac ion channels". In: New England Journal of Medicine 328.17 (1993), pp. 1244-1251.

[114] X. Li, D. Gutierrez, M. Hanson, J. Han, M. Mark, H. Chiel, P. Hegemann, L. Landmesser, and S. Herlitze. "Light activation of channelrhodopsin-2 in excitable cells of Caenorhabditis elegans triggers rapid behavioral responses". In: Curr Biol 15 (24 Dec. 2005), pp. 2279-84. DOI: 10.1016/j.cub.2005.11.032.

[115] X. Han and E. Boyden. "Multiple-color optical activation, silencing, and desynchronization of neural activity, with single-spike temporal resolution". In: PLoS One 2 (3 Mar. 2007), e299. DOI: 10.1371/journal.pone.0000299.

[116] S. Zhao, J. T. Ting, H. E. Atallah, L. Qiu, J. Tan, B. Gloss, G. J. Augustine, K. Deisseroth, M. Luo, A. M. Graybiel, et al. "Cell type-specific channelrhodopsin2 transgenic mice for optogenetic dissection of neural circuitry function". In: Nature methods 8.9 (2011), pp. 745-752.

[117] J. A. Cardin, M. Carlén, K. Meletis, U. Knoblich, F. Zhang, K. Deisseroth, L.-H. Tsai, and C. I. Moore. "Targeted optogenetic stimulation and recording of neurons in vivo using cell-type-specific expression of Channelrhodopsin-2". In: Nature protocols 5.2 (2010), p. 247.

[118] X. Han and E. Boyden. "Multiple-color optical activation, silencing, and desynchronization of neural activity, with single-spike temporal resolution". In: PLoS One 2 (3 Mar. 2007), e299. DOI: 10.1371/journal.pone.0000299. 
[119] V. Gradinaru, K. R. Thompson, and K. Deisseroth. "eNpHR: a Natronomonas halorhodopsin enhanced for optogenetic applications". In: Brain cell biology 36.1-4 (2008), pp. 129-139.

[120] A. B. Arrenberg, F. Del Bene, and H. Baier. "Optical control of zebrafish behavior with halorhodopsin". In: Proceedings of the National Academy of Sciences 106.42 (2009), pp. 17968-17973.

[121] E. Bamberg, J. Tittor, and D. Oesterhelt. "Light-driven proton or chloride pumping by halorhodopsin." In: Proceedings of the National Academy of Sciences 90.2 (1993), pp. 639-643.

[122] M. Funken, D. Malan, P. Sasse, and T. Bruegmann. "Optogenetic hyperpolarization of cardiomyocytes terminates ventricular arrhythmia". In: Frontiers in physiology 10 (2019), p. 498.

[123] T. V. Karathanos, J. D. Bayer, D. Wang, P. M. Boyle, and N. A. Trayanova. "Opsin spectral sensitivity determines the effectiveness of optogenetic termination of ventricular fibrillation in the human heart: a simulation study". In: The Journal of Physiology 594.23 (2016), pp. 6879-6891. DOI: 10.1113/JP271739. eprint: https://physoc.onlinelibrary.wiley.com/doi/pdf/10.1113/JP271739.

[124] P. Boyle, J. Williams, and C. Ambrosi. "A comprehensive multiscale framework for simulating optogenetics in the heart". In: Nat Commun 4.9 (2370 2013). DOI: $10.1038 /$ ncomms3370.

[125] E. C. A. Nyns et al. "An automated hybrid bioelectronic system for autogenous restoration of sinus rhythm in atrial fibrillation". In: Science Translational Medicine 11.481 (2019). DOI: 10.1126/scitranslmed.aau6447.

[126] E. C. Nyns, A. Kip, C. I. Bart, J. J. Plomp, K. Zeppenfeld, M. J. Schalij, A. A. de Vries, and D. A. Pijnappels. "Optogenetic termination of ventricular arrhythmias in the whole heart: towards biological cardiac rhythm management". In: European Heart Journal 38.27 (Dec. 2016), pp. 2132-2136. DOI: 10.1093/ eurheartj/ehw574. 
[127] C.-h. Luo and Y. Rudy. "A model of the ventricular cardiac action potential. Depolarization, repolarization, and their interaction." In: Circulation research 68.6 (1991), pp. 1501-1526.

[128] Y. Rudy. "Molecular basis of cardiac action potential repolarization". In: Annals of the New York Academy of Sciences 1123.1 (2008), pp. 113-118.

[129] H.-T. Shih. "Anatomy of the action potential in the heart." In: Texas Heart Institute Journal 21.1 (1994), p. 30.

[130] D. DiFrancesco. "The Role of the Funny Current in Pacemaker Activity". In: Circulation Research 106.3 (2010), pp. 434-446. DOI: 10.1161/CIRCRESAHA.109. 208041. eprint: https://www.ahajournals.org/doi/pdf/10.1161/CIRCRESAHA. 109.208041.

[131] G. Kanaporis and L. A. Blatter. "The mechanisms of calcium cycling and action potential dynamics in cardiac alternans". In: Circulation research 116.5 (2015), pp. $846-856$.

[132] F. G. Cosi, W. Giese, W. Neubert, S. Luther, N. Chamakuri, U. Parlitz, and M. Falcke. "Multiscale modeling of dyadic structure-function relation in ventricular cardiac myocytes". In: Biophysical Journal 117.12 (2019), pp. 2409-2419.

[133] Y. Tang and H. G. Othmer. "A model of calcium dynamics in cardiac myocytes based on the kinetics of ryanodine-sensitive calcium channels". In: Biophysical journal 67.6 (1994), pp. 2223-2235.

[134] F. G. Cosi. "Impact of Structure Modification on Cardiomyocyte Functionality". In: ().

[135] D. M. Bers. "Cardiac excitation-contraction coupling". In: Nature 415.6868 (2002), pp. 198-205.

[136] G. Ferrier and S. Howlett. "Cardiac excitation-contraction coupling: role of membrane potential in regulation of contraction." In: American journal of physiology. Heart and circulatory physiology 2805 (2001), H1928-44. 
[137] M. Luo and M. E. Anderson. "Mechanisms of Altered $\mathrm{Ca}<\sup >2+</ \sup >$ Handling in Heart Failure". In: Circulation Research 113.6 (2013), pp. 690-708. DOI: 10.1161/CIRCRESAHA.113.301651. eprint: https://www.ahajournals.org/ doi/pdf/10.1161/CIRCRESAHA.113.301651.

[138] Y. Haraguchi, T. Shimizu, M. Yamato, A. Kikuchi, and T. Okano. "Electrical coupling of cardiomyocyte sheets occurs rapidly via functional gap junction formation". In: Biomaterials 27.27 (2006), pp. 4765-4774.

[139] N. M. Kumar and N. B. Gilula. "The gap junction communication channel". In: Cell 84.3 (1996), pp. 381-388.

[140] M. M. Falk, L. K. Buehler, N. M. Kumar, and N. B. Gilula. "Cell-free synthesis and assembly of connexins into functional gap junction membrane channels". In: The EMBO journal 16.10 (1997), pp. 2703-2716.

[141] G. Sosinsky. "Mixing of connexins in gap junction membrane channels". In: Proceedings of the National Academy of Sciences 92.20 (1995), pp. 9210-9214.

[142] "Medical gallery of Blausen Medical 2014". en. In: WikiJournal of Medicine 1.2 (2014). DOI: $10.15347 /$ wjm/2014.010.

[143] R. Clayton, O. Bernus, E. Cherry, H. Dierckx, F. Fenton, L. Mirabella, A. Panfilov, F. Sachse, G. Seemann, and H. Zhang. "Models of cardiac tissue electrophysiology: Progress, challenges and open questions". In: Progress in Biophysics and Molecular Biology 104.1 (2011). Cardiac Physiome project: Mathematical and Modelling Foundations, pp. 22-48. DoI: https://doi.org/10. 1016/j.pbiomolbio.2010.05.008.

[144] P. C. Franzone, L. Pavarino, and B. Taccardi. "Simulating patterns of excitation, repolarization and action potential duration with cardiac bidomain and monodomain models". In: Mathematical biosciences 197.1 (2005), pp. 35-66. 
[145] J. Sundnes, B. F. Nielsen, K. A. Mardal, X. Cai, G. T. Lines, and A. Tveito. "On the computational complexity of the bidomain and the monodomain models of electrophysiology". In: Annals of biomedical engineering 34.7 (2006), pp. 10881097.

[146] M. Potse, B. Dubé, J. Richer, A. Vinet, and R. M. Gulrajani. "A comparison of monodomain and bidomain reaction-diffusion models for action potential propagation in the human heart". In: IEEE Transactions on Biomedical Engineering 53.12 (2006), pp. 2425-2435.

[147] B. J. Roth and J. P. Wikswo. "A bidomain model for the extracellular potential and magnetic field of cardiac tissue". In: IEEE Transactions on Biomedical Engineering 4 (1986), pp. 467-469.

[148] D. Barkley. "A model for fast computer simulation of waves in excitable media". In: Physica D: Nonlinear Phenomena 49.1-2 (1991), pp. 61-70.

[149] D. Barkley. "Barkley model". In: Scholarpedia 3.11 (2008), p. 1877.

[150] A. Panfilov and A. Rudenko. "Two regimes of the scroll ring drift in the threedimensional active media”. In: Physica D: Nonlinear Phenomena 28.1-2 (1987), pp. 215-218.

[151] K. H. ten Tusscher, D. Noble, P.-J. Noble, and A. V. Panfilov. "A model for human ventricular tissue". In: American Journal of Physiology-Heart and Circulatory Physiology 286.4 (2004), H1573-H1589.

[152] C.-h. Luo and Y. Rudy. "A dynamic model of the cardiac ventricular action potential. I. Simulations of ionic currents and concentration changes." In: Circulation research 74.6 (1994), pp. 1071-1096.

[153] E. M. Izhikevich. "Simple model of spiking neurons". In: IEEE Transactions on Neural Networks 14.6 (2003), pp. 1569-1572. DOI: 10.1109/TNN.2003.820440.

[154] E. Neher and B. Sakmann. "The patch clamp technique". In: Scientific American 266.3 (1992), pp. 44-51. 
[155] A. Molleman. Patch clamping: an introductory guide to patch clamp electrophysiology. John Wiley \& Sons, 2003.

[156] B. Sakmann and E. Neher. "Patch clamp techniques for studying ionic channels in excitable membranes". In: Annual review of physiology 46.1 (1984), pp. 455472.

[157] B. G. Kornreich. "The patch clamp technique: principles and technical considerations". In: Journal of Veterinary Cardiology 9.1 (2007), pp. 25-37.

[158] A. L. Hodgkin and A. F. Huxley. "A quantitative description of membrane current and its application to conduction and excitation in nerve". In: The Journal of physiology 117.4 (1952), p. 500.

[159] D. Noble. "A modification of the Hodgkin-Huxley equations applicable to Purkinje fibre action and pacemaker potentials". In: The Journal of physiology 160.2 (1962), p. 317.

[160] D. Noble. "Electrical properties of cardiac muscle attributable to inward going (anomalous) rectification". In: Journal of Cellular and Comparative Physiology 66.S2 (1965), pp. 127-135.

[161] D. Noble. "Modeling the heart-from genes to cells to the whole organ". In: Science 295.5560 (2002), pp. 1678-1682.

[162] D. Noble. "The rise of computational biology". In: Nature Reviews Molecular Cell Biology 3.6 (2002), pp. 459-463.

[163] K. H. W. J. ten Tusscher, D. Noble, P. J. Noble, and A. V. Panfilov. "A model for human ventricular tissue". en. In: American Journal of Physiology-Heart and Circulatory Physiology 286.4 (2004), H1573-H1589. DOI: 10.1152/ajpheart. 00794.2003 .

[164] M. Courtemanche, R. J. Ramirez, and S. Nattel. "Ionic mechanisms underlying human atrial action potential properties: insights from a mathematical model". 
In: American Journal of Physiology-Heart and Circulatory Physiology 275.1 (1998), H301-H321.

[165] S. F. Pravdin, V. I. Berdyshev, A. V. Panfilov, L. B. Katsnelson, O. Solovyova, and V. S. Markhasin. "Mathematical model of the anatomy and fibre orientation field of the left ventricle of the heart". In: Biomedical engineering online 12.1 (2013), p. 54.

[166] D. Noble. Multilevel modelling in Systems Biology: From Cells to Whole Organs In: Szallasi Z, Periwal V, Stelling J (Eds). System, modelling in Cellular Biology. 2006.

[167] N. A. Trayanova. "Whole-heart modeling: applications to cardiac electrophysiology and electromechanics". In: Circulation research 108.1 (2011), pp. 113128.

[168] N. Smith, P. Mulquiney, M. P. Nash, C. P. Bradley, D. Nickerson, and P. Hunter. "Mathematical modelling of the heart: cell to organ". In: Chaos, Solitons $\&$ Fractals 13.8 (2002), pp. 1613-1621.

[169] V. E. Bondarenko, G. P. Szigeti, G. C. L. Bett, S.-J. Kim, and R. L. Rasmusson. "Computer model of action potential of mouse ventricular myocytes". In: American Journal of Physiology-Heart and Circulatory Physiology 287.3 (2004), H1378-H1403. DOI: 10.1152/ajpheart.00185.2003.

[170] P. Hegemann, S. Ehlenbeck, and D. Gradmann. "Multiple photocycles of channelrhodopsin". In: Biophysical journal 89.6 (2005), pp. 3911-3918.

[171] K. Nikolic, N. Grossman, M. S. Grubb, J. Burrone, C. Toumazou, and P. Degenaar. "Photocycles of channelrhodopsin-2". In: Photochemistry and photobiology 85.1 (2009), pp. 400-411.

[172] N. Grossman, K. Nikolic, C. Toumazou, and P. Degenaar. "Modeling study of the light stimulation of a neuron cell with channelrhodopsin-2 mutants". In: IEEE Transactions on Biomedical Engineering 58.6 (2011), pp. 1742-1751. 
[173] S. S. Talathi, P. R. Carney, and P. P. Khargonekar. "Control of neural synchrony using channelrhodopsin-2: a computational study". In: Journal of computational neuroscience 31.1 (2011), pp. 87-103.

[174] G. Nagel, T. Szellas, W. Huhn, S. Kateriya, N. Adeishvili, P. Berthold, D. Ollig, P. Hegemann, and E. Bamberg. "Channelrhodopsin-2, a directly light-gated cation-selective membrane channel". In: Proceedings of the National Academy of Sciences 100.24 (2003), pp. 13940-13945.

[175] J. C. Williams, J. Xu, Z. Lu, A. Klimas, X. Chen, C. M. Ambrosi, I. S. Cohen, and E. Entcheva. "Computational optogenetics: empirically-derived voltageand light-sensitive channelrhodopsin-2 model". In: PLoS computational biology 9.9 (2013), e1003220. DOI: 10.1371/journal.pcbi.1003220.

[176] J. Wong, O. J. Abilez, and E. Kuhl. "Computational optogenetics: a novel continuum framework for the photoelectrochemistry of living systems". In: Journal of the Mechanics and Physics of Solids 60.6 (2012), pp. 1158-1178.

[177] P. M. Boyle, J. C. Williams, E. Entcheva, and N. A. Trayanova. "A computational framework for simulating cardiac optogenetics". In: 2012 Computing in Cardiology. IEEE. 2012, pp. 5-8.

[178] O. Abilez, J. Wong, R. Prakash, K. Deisseroth, C. Zarins, and E. Kuhl. "Multiscale Computational Models for Optogenetic Control of Cardiac Function". In: Biophysical Journal 101.6 (2011), pp. 1326-1334. DOI: https://doi.org/10.1016/ j.bpj.2011.08.004.

[179] S. P. Thomas, L. Bircher-Lehmann, S. A. Thomas, J. Zhuang, J. E. Saffitz, and A. G. Kléber. "Synthetic strands of neonatal mouse cardiac myocytes: structural and electrophysiological properties". In: Circulation research 87.6 (2000), pp. 467-473.

[180] Y.-S. Lee, J.-S. Song, M. Hwang, B. Lim, B. Joung, and H.-N. Pak. "A new efficient method for detecting phase singularity in cardiac fibrillation". In: PLoS one 11.12 (2016), e0167567. 
[181] R. A. Gray, A. M. Pertsov, and J. Jalife. "Spatial and temporal organization during cardiac fibrillation". In: Nature 392.6671 (1998), pp. 75-78.

[182] H.-N. Pak, Y.-B. Liu, H. Hayashi, Y. Okuyama, P.-S. Chen, and S.-F. Lin. "Synchronization of ventricular fibrillation with real-time feedback pacing: implication to low-energy defibrillation". In: American Journal of Physiology-Heart and Circulatory Physiology 285.6 (2003), H2704-H2711.

[183] K. Umapathy, K. Nair, S. Masse, S. Krishnan, J. Rogers, M. P. Nash, and K. Nanthakumar. "Phase mapping of cardiac fibrillation". In: Circulation: Arrhythmia and Electrophysiology 3.1 (2010), pp. 105-114.

[184] A. N. Iyer and R. A. Gray. "An experimentalist's approach to accurate localization of phase singularities during reentry". In: Annals of biomedical engineering 29.1 (2001), pp. 47-59.

[185] M.-A. BRAY, S.-F. LIN, R. R. Aliev, B. J. Roth, and J. P. Wikswo Jr. "Experimental and theoretical analysis of phase singularity dynamics in cardiac tissue". In: Journal of cardiovascular electrophysiology 12.6 (2001), pp. 716-722.

[186] M. J. Killeen, G. Thomas, I. Gurung, C. Goddard, J. Fraser, M. Mahaut-Smith, W. Colledge, A. Grace, and C.-H. Huang. "Arrhythmogenic mechanisms in the isolated perfused hypokalaemic murine heart". In: Acta Physiologica 189.1 (2007), pp. 33-46.

[187] A. A. Wilde. "K+ ATP-channel opening and arrhythmogenesis". In: Journal of cardiovascular pharmacology 24 (1994), S35-S40.

[188] A. V. Glukhov, T. P. Flagg, V. V. Fedorov, I. R. Efimov, and C. G. Nichols. "Differential KATP channel pharmacology in intact mouse heart". In: Journal of molecular and cellular cardiology 48.1 (2010), pp. 152-160.

[189] S. Grill, V. S. Zykov, and S. C. Müller. "Feedback-Controlled Dynamics of Meandering Spiral Waves". In: Phys. Rev. Lett. 75 (18 Oct. 1995), pp. 33683371. DOI: 10.1103/PhysRevLett.75.3368. 
[190] P. S. Petkova-Kirova, B. London, G. Salama, R. L. Rasmusson, and V. E. Bondarenko. "Mathematical modeling mechanisms of arrhythmias in transgenic mouse heart overexpressing TNF- $\alpha$ ". In: American Journal of Physiology-Heart and Circulatory Physiology 302.4 (2012), H934-H952. DOI: 10.1152/ajpheart. 00493.2011.

[191] V. Krinski. "Fibrillation in the excitable media". In: Problemy Kibernetiki 2 (1 1968), pp. 59-80.

[192] J. M. Davidenko, P. Kent, D. R. Chialvo, D. C. Michaels, and J. Jalife. "Sustained vortex-like waves in normal isolated ventricular muscle". In: Proc. Natl. Acad. Sci. USA. 87 (1990), pp. 8785-8789. DOI: https://doi.org/10.1073/pnas.87.22.8785.

[193] J. M. Davidenko, A. M. Pertsov, R. Salomonsz, W. Baxter, and J. Jalife. "Stationary and drifting spiral waves of excitation in isolated cardiac muscle". In: Nature 355 (1991), pp. 349-351. DOI: doi:10.1038/355349a0.

[194] R. Ader, J. Weijnen, and P. Moleman. "Retention of a passive avoidance response as a function of the intensity and duration of electric shock". In: Psychonomic Science 26.3 (1972), pp. 125-128.

[195] M. Ramsey. Advanced automatic external defibrillator powered by alternative and optionally multiple electrical power sources and a new business method for single use AED distribution and refurbishment. US Patent App. 10/348,564. July 2004.

[196] S.-J. Woo, J. H. Hong, T. Y. Kim, B. W. Bae, and K. J. Lee. "Spiral wave drift and complex-oscillatory spiral waves caused by heterogeneities in twodimensional in vitro cardiac tissues". In: New Journal of Physics 10.1 (2008), p. 015005.

[197] A. Panfilov and B. Vasiev. "The drift of a vortex in an inhomogeneous system of two coupled fibers". In: Chaos, Solitons \& Fractals 1.2 (1991), pp. 119-129. 
[198] V. Biktashev and A. Holden. "Resonant drift of autowave vortices in two dimensions and the effects of boundaries and inhomogeneities". In: Chaos, Solitons Fractals 5.3 (1995). Nonlinear Phenomena in Excitable Physiological Systems, pp. 575-622. DOI: https://doi.org/10.1016/0960-0779(93)E0044-C.

[199] V. N. Biktashev and A. V. Holden. "Resonant drift of an autowave vortex in a bounded medium". In: Physics Letters A 181.3 (Oct. 1993), pp. 216-224. DOI: 10.1016/0375-9601(93)90642-D.

[200] I. V. Biktasheva, Y. E. Elkin, and V. N. Biktashev. "Resonant drift of spiral waves in the complex Ginzburg-Landau equation". In: Journal of biological physics 25.2-3 (1999), pp. 115-127.

[201] V. A. Davydov, V. Zykov, A. S. Mikhailov, and P. K. Brazhnik. "Drift and resonance of helical waves in distributed active media". In: Radiophysics and Quantum Electronics 31 (1988), pp. 419-426.

[202] V. S. Zykov, A. S. Mikhailov, and S. C. Müller. "Controlling Spiral Waves in Confined Geometries by Global Feedback". In: Phys. Rev. Lett. 78 (17 Apr. 1997), pp. 3398-3401. DOI: 10.1103/PhysRevLett.78.3398.

[203] V. Zykov and H. Engel. "Feedback-mediated control of spiral waves". In: Physica D: Nonlinear Phenomena 199.1 (2004). Trends in Pattern Formation: Stability , Control and Fluctuations, pp. 243-263. DOI: https://doi.org/10.1016/j.physd. 2004.10.001.

[204] J. Schlesner, V. Zykov, H. Brandtstädter, I. Gerdes, and H. Engel. "Efficient control of spiral wave location in an excitable medium with localized heterogeneities". In: New Journal of Physics 10.1 (2008), p. 015003.

[205] K. Deisseroth. "Optogenetics". In: NATURE METHODS 8 (1 Jan. 2011), pp. 2629. DOI: 10.1038/NMETH.F.324. 
[206] I. Feola, L. Volkers, R. Majumder, A. Teplenin, M. J. Schalij, A. V. Panfilov, A. A. de Vries, and D. A. Pijnappels. "Localized Optogenetic Targeting of Rotors in Atrial Cardiomyocyte Monolayers". In: Circulation: Arrhythmia and Electrophysiology 10.11 (2017), e005591. DOI: 10.1161/CIRCEP.117.005591.

[207] S. Hussaini et al. "Drift and termination of spiral waves in optogenetically modified cardiac tissue at sub-threshold illumination". In: bioRxiv (2020). DOI: 10.1101/2020.06.12.148734. eprint: https://www.biorxiv.org/content/early/ 2020/06/13/2020.06.12.148734.full.pdf.

[208] A. Mikhailov, V. Davydov, and V. Zykov. "Complex dynamics of spiral waves and motion of curves". In: Physica D: Nonlinear Phenomena 70.1 (1994), pp. 139. DOI: https://doi.org/10.1016/0167-2789(94)90054-X.

[209] V. N. Biktashev. "Resonance and feedback strategies for low-voltage defibrillation". In: Cardiac Bioelectric Therapy. Springer, 2009, pp. 493-510.

[210] V. N. Biktashev and A. V. Holden. "Design principles of a low voltage cardiac defibrillator based on the effect of feedback resonant drift." In: Journal of theoretical biology 1692 (1994), pp. 101-12.

[211] S. Morgan, G. Plank, I. Biktasheva, and V. Biktashev. "Low energy defibrillation in human cardiac tissue: a simulation study". In: Biophys J 96.4 (2009), pp. 13641373. DOI: $10.1016 / j . b p j .2008 .11 .031$.

[212] S. Kharche, I. Biktasheva, G. Seemann, H. G. Zhang, and V. Biktashev. "Cardioversion using feedback stimuli in human atria". In: 2012 Computing in Cardiology. IEEE. 2012, pp. 133-136.

[213] G. H. Bardy and R. Mehra. Apparatus for automatically inducing fibrillation. US Patent 5,129,392. July 1992.

[214] P. Sasse, M. Funken, T. Beiert, and T. Bruegmann. "Termination of Cardiac Arrhythmia: Mechanistic Enlightenment and Therapeutic Application?" In: Front Physiol 6 (10 June 2019), p. 675. DOI: 10.3389/fphys.2019.00675. 
[215] D. P. Zipes, J. Fischer, R. M. King, A. [ Nicoll], and W. W. Jolly. "Termination of ventricular fibrillation in dogs by depolarizing a critical amount of myocardium". In: The American Journal of Cardiology 36.1 (1975), pp. 37-44. DOI: https: //doi.org/10.1016/0002-9149(75)90865-6.

[216] W. Quan and Y. Rudy. "Unidirectional block and reentry of cardiac excitation: a model study." In: Circulation Research 66.2 (1990), pp. 367-382. DOI: 10. 1161/01.RES.66.2.367. eprint: https://www.ahajournals.org/doi/pdf/10.1161/ 01.RES.66.2.367.

[217] R. M. Shaw and Y. Rudy. "The vulnerable window for unidirectional block in cardiac tissue: characterization and dependence on membrane excitability and intercellular coupling". In: Journal of cardiovascular electrophysiology 6.2 (1995), pp. 115-131.

[218] S. M. Dillon. "Optical recordings in the rabbit heart show that defibrillation strength shocks prolong the duration of depolarization and the refractory period." In: Circulation Research 69.3 (1991), pp. 842-856. DOI: 10.1161/01.RES.69.3.842. 


\section{Acknowledgements}

Researching and writing a doctoral thesis would not have been possible for me without the support of many people. I would like to thank them all very much!

Foremost, I would like to thank my supervisor, Prof. Dr. Stefan Luther, who gave me the opportunity to do my doctorate in the Biomedical Physics Biomedical Physics Group. Thank you for giving me the courage and freedom to get involved in many different and interesting projects. Thank you for creating such a fascinating group with a unique and stimulating atmosphere.

I would like to express my sincere gratitude to Prof. Valentin Krinski for his insightful ideas, constructive guidance, and motivating discussions.

Many thanks to Dr. Rupamanjari Majumder and Prof. Ulrich Parlitz for their great input and encouragement on my $\mathrm{PhD}$ projects. Thanks you for your valuable support during the difficult phases of my study.

In addition, I would like to thank two members of my thesis committee, Prof. Jörge Enderlein and Dr. Andreas Neef, for their scientific guidance during the thesis committee meetings. I would like to thank the members of my defence committee and especially Prof. Jörge Enderlein for reviewing my thesis.

I would like to thank my collaborators Prof. Vadim N. Biktashev, Prof. Leonardo Sacconi and Prof. Gil Bub for the fruitful discussions that gave me a different perspective on my projects. Thanks to Valentina Biasci for providing her fascinating experimental results in my study.

None of the work for this thesis would have been possible without the lab, IT, and administrative infrastructure and the continuous support of the staff members maintaining it, in particular Dr. Hendrik (Hecke) Degering, Dr. Denny Fliegner, Gerhard Nolte, Marion Kunze, Tina Althaus, and Caroline Siebert. Thanks so much!

This work would not have been possible without the ongoing exchange and fruitful cooperation and discussions with my colleagues of the Research Group Biomedical Physics. I would like to thank all of them in particular, Florian Spreckelsen, Justine Wolter, Baltasar Rufus Rüchardt, Filippo Cosi, and Thomas Lilienkamp. 
I would also like to mention Alexander Schlemmer, Raúl Quiñonez, Vineesh Kappadan, Sebastian Berg, Jan Christoph, Johannes Schöder-Schetelig, Jan Lebert, Annette Witt, Claudia Richter, and Laura Diaz Rodriguez as kind and valuable colleagues.

Many thanks go to two outstanding master students Vishalini Venkatesan and Aidai Mamyraiym kyzy for their amazing efforts and input on the experimental studies of this work. Thank you!

Thanks to Justine Wolter, Baltasar Rufus Rüchardt, Babak Nasouri, Aina GallemíPérez, Olinka Ramirez Soto, and Venecia Chávez Medina, who made the workplace friendlier and more pleasant with fun conversations during the coffee time.

My heartfelt thanks to the great people who make me feel at home here in Göttingen: Justine, Babak \& Y Yasaman, Reyhaneh, Leila, Nima, Pari $\&$ Mohsen $\&$ Milan, Ranabir \& Souporni \& Coco, and Parastoo \& Mohsen \& Armita.

At this point, I cannot find a word to thank and express my gratitude to the people who have supported me throughout my life. Mahrooseh, Masoud, Arezou \& Mohammad, I wish you could have been beside me at the day of my defense.

A special thanks from my heart to my parents, Mahrooseh $\& 5$ Masoud. 
